\title{
Exploring cognition in visual search and vigilance tasks with eye tracking and pupillometry
}

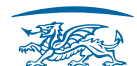

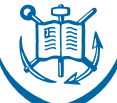 \\ Swansea University \\ Prifysgol Abertawe
}

Joel T. Martin

Department of Psychology

Swansea University

Submitted to Swansea University in fulfilment of the requirements for the Degree of

Doctor of Philosophy

January 2019 



\begin{abstract}
Recent findings in experimental psychology suggest that pupillometry, the measurement of pupil size, can provide insight into cognitive processes associated with effort and target detection in visual search tasks and monitoring performance in vigilance tasks. With the increasing availability, affordability and flexibility of video-based eye tracking hardware, these experimental findings point to lucrative practical applications such as real-time biobehavioural monitoring systems to assist with socially important tasks in operational settings. The aim of the current thesis was to explore this potential with further experimental work paying close attention to methodological issues which complicate cognitive interpretations of pupillary responses, such as physical stimulus confounds and eye movement-related measurement error in video-based systems. Six original experiments were designed to specifically explore the relationship between pupil size, cognition and behavioural performance in classic visual search and vigilance paradigms. Experiments 1-2 examined the pupillometric effects of effort and target detection in visual search with briefly presented stimuli. Pupil responses showed small variability with respect to manipulations of set size and target presence but were influenced substantially by the requirement for a motor response. Experiments 3-4 explored the cognitive pupil dynamics of free-viewing visual search with data-driven correction for eye movement artefacts. Group-level averages revealed small transient pupil dilations following fixations on targets but not distractors, an effect which was not contingent on a motor response or correction for gaze position artefacts. Experiments 5-6 looked at the relationship between pupil size and detection performance measures in two types of vigilance task. Changes in baseline and stimulus-evoked pupil responses loosely mirrored changes in performance, but the relationships were neither linear nor consistent. Overall, the thesis affirms the practical potential for using cognitive pupillometry in research and applied settings, but emphasises the constraints arising from methodological and theoretical limitations.
\end{abstract}



.. 



\section{Declaration}

This work has not previously been accepted in substance for any degree and is not being concurrently submitted in candidature for any degree.

Signed (candidate)

Date

\section{STATEMENT 1}

This thesis is the result of my own investigations, except where otherwise stated. Where correction services have been used, the extent and nature of the correction is clearly marked in footnote(s). Other sources are acknowledged by footnotes giving explicit references. A bibliography is appended.

Signed (candidate)

Date

\section{STATEMENT 2}

I hereby give consent for my thesis, if accepted, to be available for photocopying and for inter-library loan, and for the title and summary to be made available to outside organisations.

Signed (candidate)

Date 



\section{Contents}

List of Figures $\quad$ xv

$\begin{array}{lll}\text { Nomenclature } & \text { xvii }\end{array}$

1 Introduction 1

1.1 The current thesis . . . . . . . . . . . . . . . 5

2 Literature review $\quad 7$

2.1 Attention and working memory: An overview . . . . . . . . . 7

2.1 .1 Attention ................... 7

2.1.2 Working memory . . . . . . . . . . . . . 11

2.2 Vigilance . . . . . . . . . . . . . . . . . . . . 14

2.2.1 Vigilance tasks and related measures . . . . . . . . . . 14

2.2.2 Factors affecting vigilance . . . . . . . . . . . . 16

2.2.3 Contemporary theoretical accounts of vigilance . . . . . . . 18

2.2.4 Brain mechanisms of vigilant attention . . . . . . . . 21

2.2 .5 Vigilance in the real world . . . . . . . . . . . . . 21

2.3 Visual search . . . . . . . . . . . . . . . . 22

2.3.1 The visual search paradigm _............ . 23

2.3.2 Theories of visual search . . . . . . . . . . . . 24

2.3.3 Factors affecting visual search performance in the laboratory and the real world . . . . . . . . . . . . . . . . 28

2.3 .4 Debated issues . . . . . . . . . . . . . . . 32

2.4 Eye-tracking and eye movements . . . . . . . . . . . . . . 34

2.4.1 Eye movements and measures . . . . . . . . . . 35

2.4.2 Eye tracking in psychological research . . . . . . . . . . 36

2.4.3 Interactive applications of video-based eye tracking . . . . . . 39

2.5 Pupillometry . . . . . . . . . . . . . . . . . 40 
2.5.1 Cognitive modulation of the pupillary light response (PLR) . . 40

2.5.2 Cognitive factors linked to the psychosensory pupil response . 41

2.5.3 Neural mechanisms of pupil control . . . . . . . . . . . . . . 46

2.5.4 Practical applications of cognitive pupillometry . . . . . . . 48

2.5.5 Pupillometry in vigilance and visual search . . . . . . . . 53

3 General methodology

3.1 Introduction . . . . . . . . . . . . . . . . . . 55

3.2 Eye-tracking methods . . . . . . . . . . . . . . . 55

3.2.1 Infrared video-based eye tracking . . . . . . . . . . 55

3.2.2 The EyeLink 1000 system . . . . . . . . . . . . . 56

3.2.3 Saccadic thresholds and event parsing . . . . . . . . . . 57

3.2.4 Hardware configuration and experiment programming . . . . 57

3.2.5 Calibration, validation, drift checking . . . . . . . . 58

3.2.6 Data output and handling . . . . . . . . . . . . . 59

3.3 Pupillometry methods . . . . . . . . . . . . . . . . . . 59

3.3 .1 Technical issues . . . . . . . . . . . . . . 59

3.3 .2 Data processing . . . . . . . . . . . . 61

3.3.3 Statistical analysis . . . . . . . . . . . . . 65

3.3.4 General procedure for cluster-based nonparametric permutation tests . . . . . . . . . . . . . . . . 68

3.4 Summary and overview of experimental chapters . . . . . . . . . . . 69

4 Pupillometric effects of effort and target detection in visual search $\quad 71$

4.1 General introduction . . . . . . . . . . . . . . 71

4.1.1 Pupillometric effects of effort in visual search . . . . . . . . 72

4.1.2 Pupillometric effects of target detection in visual search . . . 73

4.1.3 Summary and current direction . . . . . . . . . . . . . 75

4.2 Experiment $1 \ldots \ldots \ldots 7$. . . . . . . . . . . . . 77

4.2.1 Introduction . . . . . . . . . . . . . . 77

4.2 .2 Method ...................... 78

4.2 .3 Results .................... 82

4.2 .4 Discussion ..................... 86

4.3 Experiment $2 \ldots \ldots \ldots \ldots$. . . . . . . . . . . . 87

4.3 .1 Introduction . . . . . . . . . . . . . 87

4.3 .2 Method ..................... 88 
4.3.3 Results ....................... 93

4.3 .4 Discussion ...................... 95

4.4 Overall discussion . . . . . . . . . . . . . . . . . 98

5 Pupillometry with foreshortening correction in free-viewing visual search 101

5.1 General introduction . . . . . . . . . . . . . . . 101

5.1.1 Pupil foreshortening and corrective procedures . . . . . . . 102

5.1.2 'Whole-trial' analysis of pupil data . . . . . . . . . . . . . . 104

5.1.3 Fixation-aligned pupillary response averaging . . . . . . . . 106

5.1.4 Summary and current direction . . . . . . . . . . . . . 107

5.2 Experiment $3 \ldots \ldots \ldots \ldots$. . . . . . . . . . . . 108

5.2 .1 Introduction . . . . . . . . . . . . . . . . 108

5.2 .2 Method ..................... 109

5.2 .3 Results ..................... 111

5.2 .4 Discussion . . . . . . . . . . . . . . 111

5.3 Experiment $4 \ldots \ldots \ldots \ldots \ldots \ldots$

5.3 .1 Introduction . . . . . . . . . . . . . . 115

5.3.2 Method .................... 116

5.3 .3 Results . . . . . . . . . . . . . . . . . 124

5.3 .4 Discussion . . . . . . . . . . . . . . . . 129

5.4 Overall discussion . . . . . . . . . . . . . . . . 135

6 Pupil size and performance measures during vigilance tasks 137

6.1 General introduction . . . . . . . . . . . . . . . . . 137

6.1 .1 Pupillometry and PVTs . . . . . . . . . . . . . 138

6.1.2 Pupillometry and standard vigilance tasks . . . . . . . . . 141

6.1 .3 Other relevant findings . . . . . . . . . . . . . . 143

6.1.4 Summary and current direction . . . . . . . . . . . . . . . 146

6.2 Experiment $5 \ldots \ldots \ldots \ldots \ldots$. . . . . . . . . . . . . . . . . . . .

6.2 .1 Introduction . . . . . . . . . . . . . . . 147

6.2 .2 Method ...................... 148

6.2 .3 Results . . . . . . . . . . . . . . . 152

6.2 .4 Discussion . . . . . . . . . . . . . 156

6.3 Experiment $6 \ldots \ldots \ldots \ldots$. . . . . . . . . . . . . . . . . . . .

6.3 .1 Introduction . . . . . . . . . . . . . . 160

6.3 .2 Method ....................... 160 
6.3 .3 Results . . . . . . . . . . . . . . . . . 164

6.3 .4 Discussion ..................... 168

6.4 Overall discussion . . . . . . . . . . . . . . . . . . . . 173

7 General discussion $\quad \mathbf{1 7 5}$

7.1 Introduction . . . . . . . . . . . . . . . . 175

7.1.1 Summary of introductory chapters . . . . . . . . . . . . 175

7.1.2 Summary of experimental chapters . . . . . . . . . . . 176

7.1.3 Current focus . . . . . . . . . . . . . . . . . 178

7.2 Methodological issues and recommendations . . . . . . . . . . 179

7.2.1 Stimulus confounds . . . . . . . . . . . . . . . . . . . 179

7.2.2 The pupil foreshortening error . . . . . . . . . . . . . . 182

7.2 .3 Other considerations . . . . . . . . . . . . . . . . 184

7.3 Theoretical context of experimental findings . . . . . . . . . . 185

7.3.1 Theories of LC-NA function . . . . . . . . . . . . . . 185

7.3.2 Experimental findings in context . . . . . . . . . . . 188

7.3.3 Theoretical conclusion . . . . . . . . . . . . . . . 191

7.4 Future research . . . . . . . . . . . . . . . . . . . 192

7.4.1 Quantifying and correcting for the effects of motor-response force on pupil dilation . . . . . . . . . . . . . 192

7.4.2 Determining the precise neural mechanisms of the cognitive pupillary response . . . . . . . . . . . . . . 193

7.5 Final remarks . . . . . . . . . . . . . . . . . . . 193

$\begin{array}{lll}\text { Appendix A Experiment 0: Measuring the PLR } & 195\end{array}$

$\begin{array}{lll}\text { Appendix B Departmental ethics applications } & 201\end{array}$

$\begin{array}{lr}\text { References } & 217\end{array}$ 


\section{Acknowledgements}

This thesis was supported by a grant from the Dstl, for which I am grateful. I'm also grateful to Steve for inspiring me to learn to write code (and for equipping me with an amusing arsenal of expletives to facilitate that process), but more generally for his exceptional overlordship ${ }^{1}$, which afforded me the freedom and space that I needed to work on the project, and to grow. Many others who helped and shared their time also have my gratitude, but special glowing thanks go to Sam for days of wonder (and for helpful comments on most of what follows), Panos for being a great flatmate, Seb and Kathrin for banners, bunting and rooting hormone, Greg and Weike for their ears (Weike again for her melodious voice), the mountain bike crew for memorable moments of hilarity, injury and getting bloody soaked, Tom and Mark (a.k.a. team FLANGE) for their nimble, gyroscopic, lycra-adorned camaraderie, Hiccup and Toothless for 'test drive', and Mam for keeping me well ensconced during the write-up. It's been a bit of an odyssey, but that's alright 



\section{List of Figures}

2.1 Example stimuli for feature and conjunction search . . . . . . . 25

3.1 EyeLink 1000 system and user interface . . . . . . . . . . . . 56

3.2 Configuration of eye tracking hardware . . . . . . . . . . 58

3.3 Processing pupillometry data . . . . . . . . . . . . . . 62

3.4 Schematic examples of blink interpolation . . . . . . . . . . 63

3.5 Cluster-based nonparametric permutation statistics (example) . . . . . 67

4.1 Experiment 1: Stimuli . . . . . . . . . . . . . . . . . 79

4.2 Experiment 1: Trial sequence . . . . . . . . . . . 81

4.3 Experiment 1: Main effects . . . . . . . . . . . . . . 83

4.4 Experiment 1: Signal detection measures . . . . . . . . . . . . . . 84

4.5 Experiment 1: Pupil responses . . . . . . . . . . . . . . . . 85

4.6 Experiment 1: Interaction between Target and Set Size . . . . . . . . 85

4.7 Experiment 2: Stimuli . . . . . . . . . . . . . . . . 89

4.8 Experiment 2: Trial sequence . . . . . . . . . . . . . . 91

4.9 Experiment 2: Main effects . . . . . . . . . . . . . . . . . . 94

4.10 Experiment 2: Signal detection measures . . . . . . . . . . . . . 95

4.11 Experiment 2: Pupil responses . . . . . . . . . . . . . . . 96

5.1 Experiment 3: Stimuli _. . . . . . . . . . . . . . . 110

5.2 Experiment 3: Pupil size as a function of gaze position . . . . . . . 112

5.3 Experiment 3: Pupil size as a function of time . . . . . . . . . . 113

5.4 Experiment 4: Stimuli . . . . . . . . . . . . . . 118

5.5 Experiment 4: Trial sequence . . . . . . . . . . . . . 120

5.6 Experiment 4: Example trial data with image and interest area overlay 121

5.7 Experiment 4: Frequency distributions for target and distractor fixations 123

5.8 Experiment 4: Performance data . . . . . . . . . . . . . . 125 
5.9 Experiment 4: Whole-trial pupil responses . . . . . . . . . . . . . 126

5.10 Experiment 4: Average gaze patterns during search . . . . . . . . . 127

5.11 Experiment 4: Effect of self-termination on pupil size at search offset 127

5.12 Experiment 4: Group-level effect of target vs. distractor fixation . . . 129

5.13 Experiment 4: Participant variability of target vs. distractor fixation . 130

5.14 Experiment 4: Single-trial variability of target vs. distractor fixation . 131

5.15 Experiment 4: Group-level effects of target vs. distractor refixation . . 131

5.16 Experiment 4: Group-level effect of Discovery Order . . . . . . . . . 132

6.1 Experiment 5: Stimuli and trial sequence . . . . . . . . . . . . . . . 149

6.2 Experiment 5: RT distributions . . . . . . . . . . . . . . 153

6.3 Experiment 5: Performance and pupil data . . . . . . . . . . . 154

6.4 Experiment 5: Response-locked pupil size as a function of Block . . . 155

6.5 Experiment 5: Pupil measures for the fastst and slowest 20\% responses 156

6.6 Experiment 6: Stimuli and trial sequence . . . . . . . . . . . . . . 162

6.7 Experiment 6: RT data . . . . . . . . . . . . . . . . . . 166

6.8 Experiment 6: Performance measures . . . . . . . . . . . . . 167

6.9 Experiment 6: Event- and response-locked pupil data . . . . . . . . 168

6.10 Experiment 6: Event- and response-locked pupil averages across Task Period . . . . . . . . . . . . . . . . . . . . . . 169

A.1 Experiment 0: Stimuli . . . . . . . . . . . . . . . . . . . 197

A.2 Experiment 0: Measuring the PLR . . . . . . . . . . . . . . . 198

A.3 Cluster-based non-parametric permutation statistics (example) . . . . 198 


\title{
Nomenclature
}

\author{
Acronyms / Abbreviations
}

ACh acetylcholine

ANOVA analysis of variance

ASCII American standardised code for information interchange

BCI brain-computer interface

EEG electroencephalography

ERP event-related potential

EW Edinger-Westphal

FIT feature integration theory

fMRI functional magnetic resonance imaging

HCI human-computer interaction

LC locus coeruleus

LTM long-term memory

NA noradrenalin

PFE pupil foreshortening error

PLR pupillary light response

PPR psychosensory pupil response

PVT psychomotor vigilance task

RSVP rapid serial visual presentation 
RT response time

SC superior colliculus

SDT signal detection theory

SEM standard error of the mean

SOA stimulus onset asynchrony

STM short-term memory

VWM visual working memory

WM working memory 


\section{Chapter 1}

\section{Introduction}

Since the dawn of agriculture, our species' success has come to depend increasingly on the development of tools and the mechanisation of tasks which support its needs. In recent years, the escalation of this trend has brought about a series of industrial and technological revolutions which put machines at the heart of developed societies. For the most part, machines are useful and dependable, but humans are still key to their effective design and use. Maintaining and enhancing a healthy symbiosis between human and machine is one of the great challenges faced by modern civilisation, and is likely to prove crucial to its sustainable development (Vinge, 1993).

The need to understand the intricacies of human performance when operating machinery was a major impetus for the cognitive revolution of the 1950s, which brought together what are now known collectively as the cognitive sciences-artificial intelligence, neuroscience, anthropology, linguistics, philosophy and psychology-in a multidisciplinary approach to the study of mind and brain (Miller, 2003). The effects of the cognitive revolution were especially pronounced in the discipline of psychology, where the dominant school of behaviourist thought was eclipsed by the emerging cognitivist approach to the mind. The cognitivist ideology holds that the brain is a complex information processing system, rather like a digital computer, which takes input, performs calculations, produces output, and so forth. The appeal of this idea can be traced to the success of information theory (Shannon, 1948) and to intuitions about the relation of human intelligence to computation, such as those described by Alan Turing in his classic paper which explores the question 'Can machines think?' (Turing, 1950). Cognitivism legitimised the study of abstract mental processes like attention, memory, language, learning, creativity and perception, all of which behaviourism overlooked by design. To this day there is a lively tradition of research in cognitive 
Introduction

psychology which continues to advance understanding of human mental function and to drive progress and development in other disciplines with shared interests.

Early experiments in cognitive psychology made inferences about mental processes primarily on the basis of accuracy and response time (RT) measures (Neisser, 1967), but the development and increasing availability of advanced neuroscience techniques over the last 50 years has allowed researchers to gain insight into the localisation of cognitive function in the brain, and provided useful information about how these mental processes are represented. For instance, electroencephalography (EEG) and the event-related potential (ERP) technique can be used to obtain millisecond-accurate information regarding the time courses of mental processes and their approximate spatial distribution at the level of the scalp (e.g. Berger, 1929; Donchin, 1981; Luck, 2014; Walter, Cooper, Aldridge, McCallum, \& Winter, 1964); and functional magnetic resonance imaging (fMRI) can reveal with good spatial accuracy which parts of the brain are active during a given task or form of stimulation (e.g. Logothetis, 2008; Logothetis, Pauls, Augath, Trinath, \& Oeltermann, 2001; Ogawa, Lee, Kay, \& Tank, 1990; Ogawa et al., 1992).

In addition to providing unique insights into the workings of the human brain, cognitive neuroscience techniques have also found practical application in brain computer interface (BCI) systems, which utilise the real-time neurophysiological signals as a means of communicating with computer or electro-mechanical hardware (i.e. without the need for peripheral muscular activity). BCI technology is a prime example of the developing symbiosis between humans and machines. EEG-BCIs have enabled people to control the movement of a cursor (Fabiani, McFarland, Wolpaw, \& Pfurtscheller, 2004; Kostov \& Polak, 2000; Trejo, Rosipal, \& Matthews, 2006; Vidal, 1977; Wolpaw, McFarland, Neat, \& Forneris, 1991), select letters or items on a screen (Andrea et al., 2009; Furdea et al., 2009; Neuper, Müller, Kübler, Birbaumer, \& Pfurtscheller, 2003), operate neuroprosthetic devices (Bell, Shenoy, Chalodhorn, \& Rao, 2008; Lauer, Peckham, Kilgore, \& Heetderks, 2000; Wolpaw \& McFarland, 2004), and in more recent demonstrations they have facilitated collaborative decision making in groups of noncommunicating observers (Poli, Valeriani, \& Cinel, 2014; Valeriani, Poli, \& Cinel, 2016; Valeriani, Poli, Valeriani, Poli, \& Cinel, 2015). fMRI-BCIs have allowed participants to engage in voluntary self-regulation of localised brain regions through observation of their own brain activity (DeCharms et al., 2005; Johnston, Boehm, Healy, Goebel, \& Linden, 2010; Johnston et al., 2011; Weiskopf et al., 2004; Weiskopf et al., 2003), and they have also enabled researchers to reconstruct the visual experience of a participant from recordings of brain activity (Miyawaki et al., 2008; Nishimoto et al., 
2011). Such technology has already demonstrated its capacity for improving people's lives, and future developments are likely to enhance this capacity and lead to further applications in clinical and occupational settings (Lebedev \& Nicolelis, 2006).

Another technique that has shown much promise both in research and in applied settings is video oculography, an advanced form of biometric technology which combines video cameras and image processing algorithms to measure the spatial and temporal distribution of eye movements (Duchowski, 2007). Video-based eye trackers have been used extensively in domains such as psychology, neuroscience, advertising and marketing, human factors, industrial engineering and computer science for their diagnostic and interactive capabilities (Duchowski, 2002). In their typical diagnostic capacity, eye trackers are used to obtain objective and quantitative information on the visual and attentional processes of research participants, which can then be subject to post hoc, offline assessment. Although this is the most common use of eye trackers, advances in computer power and growing interest in the psychology of human-computer interaction (HCI: Card, Moran, \& Newell, 1983; Newell \& Card, 1985) have paved the way to lucrative interactive applications. Examples include eye-based interaction with computers and virtual environments (Jacob, 1991; Starker \& Bolt, 1990; Tanriverdi \& Jacob, 2000), eye typing (Majaranta \& Räihä, 2002), communication assistance in collaborative systems (Vertegaal, 1999), and gaze contingent displays which adapt intelligently to a users visual behaviour (Parkhurst \& Niebur, 2002; Reingold, Loschky, McConkie, \& Stampe, 2003).

Whilst the visual point of regard is the primary measure of eye tracking systems and the fundamental basis for the majority of their applications, another of their available measures which has received much interest in recent years is pupil size. The pupils respond primarily to light, but it has been known since antiquity that they can give insight into mental states. There are stories of ancient European merchants who, by looking closely at the pupils of customers inspecting their wares, could detect signs of an interest greater than their feigned indifference would suggest; and Renaissance courtesans who rubbed bella donna - a plant containing atropine-into their eyes, knowing it would cause their pupils to dilate and make them appear more attractive to onlookers (Sirois \& Brisson, 2014). In addition to such folk tales, there is a growing catalogue of empirical research dating back to the 1960s documenting the cognitive factors that modulate pupil size. Specifically, nonluminance mediated fluctuations in pupil size have been linked to emotional arousal (Aboyoun \& Dabbs, 1998; Hess \& Polt, 1960; Hess, Seltzer, \& Shlien, 1965), cognitive load (Beatty \& Wagoner, 1978; Hess \& Polt, 1964; Kahneman \& Beatty, 1966, 1967), anxiety (Bitsios, Szabadi, \& Bradshaw, 
Introduction

2002, 2004; Nagai, Wada, \& Sunaga, 2002; Simpson \& Molloy, 1971), attention and salience (Libby, Lacey, \& Lacey, 1973; Mathôt, Siebold, Donk, Vitu, \& Siebold, 2015; Mathôt, Umr, Dalmaijer, Grainger, \& Stigchel, 2014; Wang \& Munoz, 2014), decision making (Einhäuser, Koch, \& Carter, 2010; Richer \& Beatty, 1985; Simpson $\&$ Hale, 1969), violated expectations and surprise (Gilzenrat, Nieuwenhuis, Jepma, \& Cohen, 2010; Nuthmann \& van der Meer, 2005; Preuschoff, 't Hart, \& Einhäuser, 2011; Privitera, Renninger, Carney, Klein, \& Aguilar, 2010; Raisig, Welke, Hagendorf, \& van der Meer, 2010; Scheepers, Mohr, Fischer, \& Roberts, 2013), memory (Kahneman \& Beatty, 1966; Magliero, 1983; Poock, 1973; Võ et al., 2008), and changes in perception (Einhäuser, Stout, Koch, \& Carter, 2008; Fahle, Stemmler, \& Spang, 2011; Hupé, Lamirel, \& Lorenceau, 2009; Kloosterman et al., 2015; Naber, Einhäuser, \& Frassle, 2011). The utility of pupillometry as a research tool for gaining insight into these facets of mental function stems from the known functional association between pupil size changes under constant luminance and momemnt-to-moment activity in the locus coeruleus (Laeng, Sirois, \& Gredeback, 2012), a brainstem nucleus known to play a pivotel role in the modulation of cognition, arousal and autonomic function (AstonJones \& Cohen, 2005; Bouret \& Sara, 2005; Mittner, Hawkins, Boekel, \& Forstmann, 2016; Sara \& Bouret, 2012; Yu \& Dayan, 2005).

The robust evidence base for cognitive effects on pupil size, the increasing availability, affordability and flexibility of eye tracking systems, and the ease and unobtrusiveness with which the information can be accessed makes pupillometry a technique with unique practical potential. It has already been used with some success to gauge the 'cognitive intensity' of system operators (Marshall, 2002), to assess sleepiness (Wilhelm et al., 2001), as a tool for diagnosing and monitoring the treatment and progression of psychiatric illnesses such as schizophrenia (Steinhauer, 2002; Steinhauer \& Hakerem, 1992; Steinhauer \& Zubin, 1982), to communicate with locked-in patients (Stoll et al., 2013), and most recently, as a BCI which allows people to spell words without looking at the letters of their choosing (Mathôt, Melmi, van der Linden, \& Van der Stigchel, 2015, 2016). Recent evidence also shows that transient pupil dilation coincides with visual target detection in visual search (Klingner, 2010a; Privitera et al., 2010; Rajkowski, Majczynski, Clayton, \& Aston-Jones, 2004; Wierda, van Rijn, Taatgen, \& Martens, 2012) and that pupil fluctuations can predict lapses in sustained attention in vigilance tasks (Kristjansson, Stern, Brown, \& Rohrbaugh, 2009; Unsworth \& Robison, 2016; van den Brink, Murphy, \& Nieuwenhuis, 2016), but it is presently unclear whether these reported effects are reliable enough to bring performance-enhancing utility to real-world tasks of visual search or vigilance. 


\subsection{The current thesis}

The state-of-the art in eye tracking and pupillometry strongly suggests a practical potential beyond that which is already known and used in research and applied settings. Specifically, recent findings suggest that these methods could further our theoretical understanding of the cognitive processes involved in socially important tasks requiring vigilance or visual search, such as many of those in the domains of security (e.g. CCTV monitoring, airport baggage screening) medicine (e.g. medical image analysis), and transport (e.g. long-distance driving, air traffic control). It is also conceivable that, with advances in theory and methods, eye-tracking and pupillometric measures could serve as the basis for novel workplace assistive technologies or procedures for evaluating fitness for duty in some of the aforementioned operational settings. The broad aim of the current thesis is to explore this potential through consideration of existing literature on theoretical and methodological issues (Chapters 2, 3, 4, 5 and 6), with further experimental groundwork in the domains of visual search (Chapters 4 and 5) and vigilance (Chapter 6), and with a general discussion of key findings and their relevance in research and applied contexts (Chapter 7). 



\section{Chapter 2}

\section{Literature review}

\subsection{Attention and working memory: An overview}

Tasks involving elements of vigilance or visual search invariably tax one's ability to selectively process information in the environment and keep relevant information in a state that is accessible over time. The cognitive capacities responsible for these abilities are known as attention and working memory (WM). In psychological research, attention and $\mathrm{WM}$ are complex, multifaceted and broadly relevant theoretical constructs, distinct from each other in many respects but overlapping in others. This section prepares for detailed discussion of vigilance and visual search with a general introduction to the psychological theory of attention and WM.

\subsubsection{Attention}

In his 1890 textbook The Principles of Psychology, William James (1890, pp. 381-382) famously said:

Everyone knows what attention is. It is the taking possession by the mind, in clear and vivid form, of one out of what seem several simultaneously possible objects or trains of thought. Focalization, concentration, of consciousness are of its essence. It implies withdrawal from some things in order to deal effectively with others, and is a condition which has a real opposite in the confused, dazed, scatterbrained state which in French is called distraction, and Zerstreutheit in German.

The relevance of this quote endures because it remains consistent with that common understanding of attention that most of us acquire at a young age and maintain throughout life. We know how to respond when our attention is requested, what it 
Literature review

means to pay more attention to what we are doing and why we are thanked for our attention after long periods of listening. In modern cognitive science, however, attention has become a vast field of research, with no single satisfactory definition (Moray, 1969), no single theory to accommodate all of the experimental data (Johnston \& Dark, 1986), and much confusion in general surrounding the topic and the language that is used to discuss it (Anderson, 2011). Despite these problems, considerable progress has been made since the days of William James in understanding the functional characteristics of attention and related brain processes. This overview focuses on the highlights of this progress without dwelling to a large extent on the persistent and pervasive problems in the field.

From the perspective of modern science then, attention is not a unitary aspect of mental function but rather a network of interacting brain processes involved in the coordination of various cognitive, perceptual and motor behaviours (Parasuraman, 1998). Although there is no universal agreement on exactly how many distinct brain processes constitute attention, there is strong evidence from behavioural and neuroscience experiments that there are at least three different types with distinct functions. One influential taxonomy developed by Posner and colleagues (Fan, McCandliss, Fossella, Flombaum, \& Posner, 2005; Fan, McCandliss, Sommer, Raz, \& Posner, 2002; Petersen \& Posner, 2012; Posner \& Boies, 1971; Posner \& Petersen, 1990) argues for the existence of three distinct attention networks responsible for the roles of alerting, orienting and executive control. Another taxonomy proposed by Parasuraman and Davies (1984) recognises selection, vigilance and control as the three main varieties of attention. For the sake of clarity, and because vigilance is a key topic in this thesis, attention is here reviewed with reference to the taxonomy of Parasuraman and Davies, although it should be noted that the differences between the two taxonomies are largely in the choice of words used to describe common findings.

\section{Varieties of attention}

The first variety of attention, selection, refers to our ability to process task-relevant information in the environment when there is so much noise competing for access to our consciousness. Cherry (1953), who famously framed this as 'the cocktail party problem', was among the first to investigate selective attention. Using a dichotic listening task, where participants were presented with different messages in each ear and asked to attend only to one, he found that participants could ignore the message in the irrelevant ear with ease, to the extent that they would recall none of its content and not even notice if the speaker changed language from English to German. However, participants 
did notice if their name was spoken, if the voice changed from male to female, or if the speech was reversed, suggesting that certain properties of an unattended message are still processed in some capacity. Following the work of Cherry and based on the results from a range of similar experiments, Broadbent (1958) proposed his influential filter theory, positing that attention acts as a bottleneck to restrict the flow of sensory information through the perceptual processing stream. Though widely hailed as a useful theory, much debate has questioned where the attentional bottleneck is located in the nervous system. Broadbent (1958) originally argued that attentional selection occurs early in the processing stream to protect subsequent limited capacity processing stages from overload, but years of heated debate and rigorous experimentation have shown that attentional selection can occur at multiple stages of processing (e.g. see Johnston \& Dark, 1986; Kahneman \& Treisman, 1984; Pashler, 1998).

Vigilance, the second variety, is broadly understood to refer to an organisms ability to sustain attention over prolonged periods of time (Parasuraman \& Davies, 1982; Parasuraman, Warm, \& See, 1998; Warm \& Jerison, 1984; Warm, Parasuraman, \& Matthews, 2008), in which respect it is similar to Posner and colleagues' (Fan et al., 2005; Fan et al., 2002; Petersen \& Posner, 2012; Posner \& Boies, 1971; Posner \& Petersen, 1990) 'alertness' / 'alerting' component of attention. The systematic study of vigilance began after the Second World War as a means of gaining insight into human factors that contributed to error in high-stakes situations, a classic example being the failure of radar and sonar system operators to detect the presence of German U-boats in the bay of Biscay. Mackworth (1948, 1950), who investigated this phenomenon objectively, discovered that human performance on a monotonous watch keeping task, under conditions similar to those experienced by radar and sonar operators, declines as time spent on-task increases-what has subsequently become known as the vigilance decrement. Interest in the scientific study of vigilance has waxed and waned in the years since Mackworth's findings, but the prevalence of automation in today's industrial climate means that findings from vigilance research are likely to play an increasingly important role in guiding the design of automated systems (e.g. Parasuraman \& Riley, 1997) and in aiding the selection of appropriate personnel for assignments (e.g. Reinerman-Jones, Matthews, Langheim, \& Warm, 2011). Vigilance is a central topic in this thesis and is discussed in much further detail in Section 2.2, and also in Sections 2.4.2 and 2.5.5, where eye tracking and pupillometric studies of vigilance are considered.

The last variety of attention in Parasuraman and Davies' classification is attentional control, which is closely associated with the central executive component of WM (soon to be discussed) and Posner and colleagues' 'executive attention'. Atten- 
tional control allows people to process multiple sources of information simultaneously without compromising performance efficiency (Duncan, 1980), and to limit processing to task-relevant information. A well known example of a paradigm that highlights the function of attentional control is the Stroop Task (MacLeod, 1991; Stroop, 1935), where participants are presented with colour names in different coloured ink and must quickly name either the word or ink colour. The classic finding here is that a person takes longer to respond when reporting the ink colour if it does not match the word, but when the task is to report the word, RT is mostly unaffected by such incongruence. This delayed response on incongruent trials is thought to reflect the requirement for attentional control, which must be used to override the automatic tendency to process the written word.

\section{Visual attention}

Much of the early experimental work on attention focused exclusively on the auditory domain, but the development and canonisation of experimental paradigms for investigating visual attention, notably visual search (e.g. Duncan \& Humphreys, 1989; Treisman \& Gelade, 1980; Wolfe, Cave, \& Franzel, 1989) and spatial cueing paradigms (e.g. Averbach \& Coriell, 1961; Eriksen \& Hoffman, 1973; Posner, 1980), gradually strengthened the focus on the visual domain. Visual search is discussed in detail in Section 2.3 due to its topical relevance in the current thesis. Here the spatial cueing task and its principal finding is outlined because of its relevance to theoretical understandings of visual search and visual attention in general.

Perhaps the most typical example of a spatial cueing task is that which was described by Posner (1980), where participants fixated at the centre of a computer screen and made rapid detection responses following the appearance of a target either to the left or right of fixation position. Prior to the target's appearance, a cue-either a plus sign or an arrow-appeared for $1000 \mathrm{~ms}$ at the locus of fixation to indicate the likely position of the coming target. When the cue was the plus sign, the target could appear at both sides with equal likelihood (a nonpredictive cue), but when the cue was an arrow, the target appeared with $80 \%$ likelihood at the indicated location (a valid predictive cue) and $20 \%$ likelihood at the opposite location (an invalid predictive cue). The principal finding was that RTs were faster when participants received valid information from arrow cues as to the location of the impending target. This effect could not have been due to an eye-gaze advantage, as the subjects were told to maintain central fixation 
and electro-oculography was used to exclude trials which contained eye movements ${ }^{1}$. Posner concluded that the effect must be linked to an attentional mechanism, separate from gaze position, which shifts in response to predictive cues and confers either a processing advantage or cost on the subsequent target stimulus depending on whether it appears at the cued or uncued location. In his own words: 'Our data show that attention is not intrinsically tied to the foveal structure of the visual system nor slaved to the overt movements of the eye' (Posner, 1980, pp. 22).

The attentional mechanism which Posner's (1980) task seemed to isolate is now widely referred to as covert attention, and variations on the spatial cueing paradigm have revealed much about its nature. For instance, the cues used in the experiment just described were central symbolic cues, so the observer had to process the meaning of the cue before they could shift covert attention to the expected location of the target. However, cues can also be presented peripherally, at the actual location of the coming target, where if salient enough they produce a reflexive shift of attention. In a now classic series of experiments, Jonides (1981) compared peripheral cues with central cues and discovered that the former confer greater benefits and costs on accuracy and RT for valid and invalid trials, which suggests that they are generally more effective than central cues at drawing attention (but for later debate, see Folk, Remington, \& Johnston, 1992; Yantis, 1993).

The findings from spatial cueing experiments have contributed substantially to the idea that visual attention behaves like a spotlight, independent from the direction of gaze, which enhances the detection efficiency of events occurring within its beam (e.g. Posner, Snyder, \& Davidson, 1980). The covert attentional spotlight has become a popular metaphor to describe the action of visual attention ${ }^{2}$ and, as will be seen, is a key idea in some theories of visual search, and also has implications for the interpretation of eye tracking data.

\subsubsection{Working memory}

Memory is the aspect of cognitive function which allows us to encode, store and retrieve information. Without it we would not be able to learn languages, develop relationships, form a sense of personal identity, or indeed perform many of the tasks that are essential to our daily lives (Eysenck, 2012). Whilst various forms of memory have

\footnotetext{
${ }^{1}$ If subjects could have actually fixated the cued location, a processing advantage would have been expected on valid trials on account of the greater density of photoreceptors in the fovea compared to the rest of the retina.

${ }^{2}$ The function of visual attention has also been described metaphorically as a 'zoom-lens' (Eriksen \& James, 1986) and a 'guassian gradient' (Downing \& Pinker, 1985).
} 
been identified, influential theories have traditionally made a basic distinction between long-term memory (LTM) and short-term memory (STM) (e.g. Atkinson \& Shiffrin, 1968; Craik \& Lockhart, 1972; Waugh \& Norman, 1965). LTM has an immeasurable capacity, can persist for a whole lifetime, and is maintained by permanent and stable changes in structure and connectivity throughout the brain (Bliss \& Collingridge, 1993; Craik \& Lockhart, 1972), whereas short-term memory has a limited capacity, decays rapidly, and is mediated by transient patterns of electrical activation in prefrontal and parietal brain regions (Baddeley, 2003; Miller, 1956; Peterson \& Peterson, 1959). Intelligent behaviour is widely believed to depend on interactions between LTM and STM, but one's ability to comprehend the immediate environment, retain information about immediate past experience, solve problems, and work towards current goals is largely dependant on STM (Baddeley, 1992).

Today, the concept of STM has been largely supplanted by that of WM, which is similar in many respects but emphasises the requirement for additional processing mechanisms for making use of STM during ongoing cognitive tasks (Cowan, 2008). WM became a dominant theoretical construct when Baddeley and Hitch (1974) showed that a single STM module could not account for all types of temporary memory. The work and theorising of Baddeley and Hitch (1974) lead to the highly influential Multicomponent Model of WM (Baddeley, 1986), in which visuo-spatial and verbal-phonological representations are held in separate stores and manipulated and managed by a collection of attentional processes termed the central executive. The model was subsequently revised to incorporate a further component, the episodic buffer (Baddeley, 2000), which aimed to account for STM features that did not fall neatly into the other stores, and for the requirement that information from the subsystems be combined together and integrated with information from LTM in such a way that permits active manipulation and maintenance. As a result of the work of Baddeley and colleagues (Baddeley, 1986, 1992, 1996, 2000, 2002, 2003, 2010; Baddeley \& Hitch, 1974; Baddeley, Thomson, \& Buchanan, 1975), WM is now widely viewed as the combination of multiple distinct components working synchronously.

Although the multicomponent model has been hugely successful and widely influential, it should be noted that there are numerous other models and theories with alternative views on key issues regarding the nature of WM, such as how it relates to LTM and knowledge, whether it is a unitary or nonunitary construct, how it relates to attention and consciousness, the role it plays in the performance of complex cognitive tasks, its neurobiological basis, and the mechanisms involved in the encoding, maintenance and retrieval of information (Miyake \& Shah, 1999). Following a comprehensive 
review of 10 different models and theories of $\mathrm{WM}^{3}$, Miyake and Shah (1999) found that there were many discrepant views on key issues, but also that there were many broad points of consensus. For instance, all researchers agreed that WM was not a structurally distinct part of the mind or brain, that executive control is integral to its function, and that LTM plays a crucial role in WM performance. Based on these and other points of convergence, Miyake and Shah (1999, p. 450) proposed the following all-encompassing definition of WM:

\begin{abstract}
Working memory is those mechanisms or processes that are involved in the control, regulation, and active maintenance of task-relevant information in the service of complex cognition, including novel as well as familiar, skilled tasks. It consists of a set of processes and mechanisms and is not a fixed "place" or "box" in the cognitive architecture. It is not a completely unitary system in the sense that it involves multiple representational codes and/or different subsystems. Its capacity limits reflect multiple factors and may even be an emergent property of the multiple processes and mechanisms involved. Working memory is closely linked to LTM, and its contents consist primarily of currently activated LTM representations, but can also extend to LTM memory representations that are closely linked to activated retrieval cues and, hence, can be quickly reactivated.
\end{abstract}

\title{
Visual working memory (VWM)
}

VWM refers to the active maintenance of visual information to serve the needs of ongoing tasks (Luck \& Vogel, 2013), and is crucial for a broad range of cognitive functions (Chun \& Potter, 1995; Jolicoeur, Dell'Acqua, \& Crebolder, 2001; Wheeler \& Treisman, 2002). As noted above, Baddeley and Hitch (1974) recognised the need for a dedicated VWM subsystem in their original WM model, although this was not nearly as well characterised as the auditory subsystem, because most of the research into STM and WM up to that point had focused on the auditory modality. Work on VWM became much more common when the change detection paradigm - first introduced by Phillips (1974) — was popularised by Luck and Vogel (1997). Variations on this paradigm have lead to many insights into the capacity, stability and duration of VWM.

In a basic change detection task, participants are presented with an array of items (e.g. coloured squares, tilted bars) which they must try to remember. The memory array then disappears and is followed shortly afterwards by a probe array, which participants must judge as being either identical to, or different from, the memory array. In Luck

\footnotetext{
${ }^{3}$ Examples of other theories and models of WM reviewed by Miyake and Shah (1999) are the Embedded-Processes Model (Cowan, 1999), the Controlled-Attention Framework (Engle, Kane, \& Tuholski, 1999) and the biologically based computational model of O'Reilly, Braver, and Cohen (1999).
} 
and Vogel's (1997) experiments, participants performed this task accurately for set sizes of 1-3 items, but their performance deteriorated systematically as the set size increased from 4-12 items. Luck and Vogel were also able to rule out the possible supplementary effects of verbal memory with a dual-task condition where the additional requirement to remember two digits for the duration of each trial yielded similar results. Further to this, performance was relatively unaffected by the duration of exposure to the memory array (i.e. 100 vs. $500 \mathrm{~ms}$ ), or by the requirement to judge differences in single features of items defined by conjunctions of multiple features. On the basis of these findings, Luck and Vogel concluded that VWM capacity is strictly limited to 1-4 integrated object percepts. This severe limitation of VWM capacity is widely supported throughout the literature (e.g. Alvarez \& Cavanagh, 2004; Awh, Barton, \& Vogel, 2007; Cowan, 2010; Pashler, 1988; Rouder et al., 2008; Sperling, 1960; Todd \& Marois, 2004; Vogel, McCollough, \& Machizawa, 2005; Vogel, Woodman, \& Luck, 2001; Xu \& Chun, 2006), although it is known to vary considerably with individual differences (Luck \& Vogel, 2013; Rouder et al., 2008; Unsworth \& Engle, 2007; Vogel \& Machizawa, 2004; Vogel et al., 2005), and there is some debate as to whether capacity is best measured in discrete 'slots' or as a continuous resource (e.g. Bays \& Husain, 2008; Luck, 2008; Rouder et al., 2008; van den Berg, Shin, Chou, George, \& Ma, 2012).

\subsection{Vigilance}

In Section 2.1.1 it was noted that vigilance can be regarded as a particular variety of attention characterised by sustained alertness, and that interest in the systematic study of vigilant attention emerged over half a century ago. Today, interest in vigilance persists due to its continuing relevance in a range of practical situations and growing importance in a world of increasing automation. This section looks more closely at vigilance by exploring the kinds of task that are used to measure it in the laboratory, factors that affect performance in these tasks, theories that attempt to account for experimental findings, brain mechanisms of vigilance, and examples of vigilance tasks in the real world. The material covered provides a methodological and theoretical precedent for the experimental work of Chapter 6, which uses eye tracking, pupil and behavioural measures to learn more about cognition and performance in vigilance tasks.

\subsubsection{Vigilance tasks and related measures}

Standard vigilance tasks are characterised by relatively long and uninterrupted periods of time, during which participants must remain alert in order to detect and respond to 
critical signals in the environment (Davies, Jones, \& Taylor, 1984; Mackworth, 1970; Warm \& Jerison, 1984). In his original vigilance task, known as the 'Clock Test', Mackworth (1948) asked his participants to monitor a 6-in. black pointer on a white background as it moved in discrete steps once per second, like the ticking motion of the second hand on a clock. Twelve times every $30 \mathrm{~min}$, but at unpredictable intervals, the pointer would move twice the normal distance - a signal to which the subject had to respond by pressing a switch. This task was undertaken for $2 \mathrm{hr}$, across which time detection accuracy decreased markedly, with the steepest decline occurring between the first and second 30 min periods. Mackworth (1950) observed similar effects in two further tasks of his own design: the 'Synthetic Radar Test', which was similar to the Clock Test but more realistically simulated the visual task of radar operators, and the 'Main Listening Test', where subjects were required to listen to a series of $2 \mathrm{~s}$ tones presented at $18 \mathrm{~s}$ intervals and detect infrequent tones whose duration was slightly increased. The principal measure in each of these experiments was the number of signals that were correctly identified, otherwise known as the detection rate.

In subsequent investigations, new measures for exploring the vigilance decrement were introduced. Notably, Broadbent (1958) used detection latency to measure performance in his 'Twenty Dials Test', where subjects had to monitor twenty steam-pressure gauges for infrequent nontransient danger signals that remained present until a response was made. Here, the vigilance decrement was characterised by RTs that increased in accordance with the amount of time spent on-task. Parasuraman and Davies (1976) also showed that the false alarm rate-the number of times the observer reported a signal when in fact there was no signal—can be combined with detection rate and RT to yield useful insights into vigilance task performance. In Parasuraman and Davies' task, subjects monitored a series of circular light flashes occurring at $4 \mathrm{~s}$ intervals across a 45 min period and responded 'yes' or 'no' after each flash to say whether its luminance was slightly dimmer than usual. Using the principles of signal detection theory (SDT: Green \& Swets, 1974) ${ }^{4}$, they were able to distinguish between changes in RT that arose from shifts in (a) internal decision bias and (b) perceptual sensitivity to the signals.

The previous examples describe the general nature of standard vigilance tasks and the kind of measures that are used to gauge performance. Though there are many variations which are often designed to emulate specific operator settings, the general principles remain the same-subjects respond to infrequent signals across a length of

\footnotetext{
${ }^{4}$ Signal detection theory is an influential framework for understanding decision accuracy, first introduced to psychology by Green and Swets (1974), and since discussed in numerous books (e.g. MacMillan \& Creelman, 2005; Wickens, 2002), book chapters (e.g. MacMillan, 2001) and journal articles (e.g. Stanislaw \& Todorov, 1999; Verde, MacMillan, \& Rotello, 2006).
} 
time, usually at least $30 \mathrm{~min}$, and their detection rate, false alarm rate, RT and other derivative measures are used to make inferences about performance (e.g. Broadbent, 1954; Davies \& Krkovic, 1965; Grier et al., 2003; Helton \& Russell, 2011; Mackworth, 1948; Parasuraman, 1987; Parasuraman \& Davies, 1976). There is however another popular type of vigilance task known as the psychomotor vigilance task (PVT: Wilkinson $\&$ Houghton, 1982) which differs with respect to these principles. In this task, instead of responding to infrequent signals over a prolonged period of time, subjects have to make speeded responses to more regular signals occurring at random intervals over a short period of time, usually 10 min or less. Wilkinson and Houghton's original version of this task was administered on a small hand-held battery-powered device displaying a millisecond counter set to '000'. The subject held the device and quickly pressed a button every time the counter began to increment, which happened at intervals ranging between 1-10 s. When a response was detected, the timer would freeze for 1.5 seconds and the RT was saved before the timer reset to '000'. A variety of performance metrics can be derived from the data produced by this task, but analysis commonly focuses on mean and median RT, the fastest and slowest $10 \%$ of trials, and the proportion of 'lapses', which are usually defined as RTs greater than $500 \mathrm{~ms}$. Whereas standard vigilance tasks aim to emulate the conditions of real-world operator settings, the PVT was originally conceived as a portable means of quickly assessing declines in vigilant attention associated with sleep loss, circadian factors and other environmental stressors. A large body of research testifies to its validity for this purpose (Basner \& Dinges, 2011; Basner, Mollicone, \& Dinges, 2011; Blatter et al., 2006; Caldwell, Prazinko, \& Caldwell, 2003; Dinges et al., 1997; Graw, Kräuchi, Knoblauch, Wirz-Justice, \& Cajochen, 2004; Van Dongen \& Dinges, 2005).

\subsubsection{Factors affecting vigilance}

Research has identified a range of factors that affect vigilance task performance. These factors are many and varied, but can generally be grouped into three broad categories: task-related, environmental and individual. The present section identifies key factors from these three categories.

\section{Task-related factors}

Performance in vigilance tasks is determined to a large extent by the nature of the task itself. For instance, experimental work has revealed that steeper decrements and overall error rates are more likely in tactile or visual tasks compared to auditory 
tasks (Davies et al., 1984; Hatfield \& Loeb, 1968; Hawkes \& Loeb, 1962; Warm $\&$ Jerison, 1984), and that auditory tasks appear to be less stressful to the operator than do visual tasks (Galinsky, Warm, \& Dember, 1993). In addition to the sensory modality of the task, the physical parameters of the signal to which the subject must respond greatly influence performance. Research has shown that error rate and RT can be reduced by making the signal more conspicuous through increasing its intensity (Adams, 1956; Lisper \& Ericsson, 1972; Loeb \& Binford, 1963; Metzger, Warm, \& Senter, 1974; Warm, Epps, \& Ferguson, 1974) or duration (Adams, 1956; Baker, 1963; Warm, Loeb, \& Alluisi, 1970). Further, the probability of signal occurrence influences detection rate, but where intensity and duration effect the subject's perceptual sensitivity, signal probability is thought to exert its effect by influencing their decision criterion (Baddeley \& Colquhoun, 1969; Broadbent \& Gregory, 1965; Parasuraman \& Davies, 1976; Williges, 1971). Finally, task instructions can influence performance through the expectancies they may lead subjects to develop (Dember, Galinsky, \& Warm, 1992; Lucaccini, Freedy, \& Lyman, 1968).

\section{Environmental factors}

Of all the environmental factors that could conceivably influence vigilance task performance, the effects of interfering noise, temperature and vibration have been explored in the greatest detail. Noise in particular has received much attention, with many laboratory experiments exploring the effects of its volume, quality and regularity on vigilance tasks of varying difficulty. As Ballard (1996) pointed out, however, interactions between the characteristics of the noise and the task can produce a range of effects, and there are many inconsistent reports in the literature. Two examples of more consistent findings at opposite extremes are that quiet varied noise like conversation or music can facilitate performance in tasks with low demand (Blackwell \& Belt, 1971; Davenport, 1972; Hancock, 1984; Kirk \& Hecht, 1963), but loud and continuous static noise (e.g. 'white' noise) contributes to poorer performance in tasks requiring a high level of processing (Broadbent, 1953, 1954; Hancock, 1984; Jerison, 1959). Regarding temperature, sudden exposure to heat can facilitate vigilance, but prolonged exposure to temperatures above $32{ }^{\circ} \mathrm{C}$ leads to increased error rates and steeper performance decrements (Hancock, 1984); and exposure to moderate or extreme cold increases the number of detection errors (Enander, 1987; Hancock, 1984). Finally, vertical vibration, when simulated with a vibrating platform at $5 \mathrm{~Hz}$, has been reported to improve vigilance performance (Shoenberger, 1967; Wilkinson \& Gray, 1974), but as noted by Poulton (1977), this effect was probably mediated by arousal. 


\section{Individual factors}

The physiological state of an individual and their disposition towards the task are important determinants of vigilance performance. The effects of being in a sleep deprived state are particularly well documented, with a large body of research showing that total and partial sleep deprivation can increase error rates and RTs in both standard vigilance tasks (Poulton \& Edwards, 1974; Wilkinson, 1960; Williams, Lubin, \& Goodnow, 1959) and PVTs (Basner \& Dinges, 2011; Basner et al., 2011; Blatter et al., 2006; Caldwell et al., 2003; Dinges et al., 1997; Graw et al., 2004; Van Dongen \& Dinges, 2005; Wilkinson \& Houghton, 1982). Rather than decreasing overall performance, the effects of sleep deprivation are thought to be manifest in the form of intermittent lapses in efficiency occurring amid periods of normal functioning (Broadbent \& Gregory, 1963). Performance efficiency is also reliably affected by circadian factors relating to the time of day (Colquhoun, 1971), which vary depending on whether the individual is a strong morning or an evening 'type' (Horne, Brass, \& Pettitt, 1980). States conducive to vigilance can also be induced or sustained by drugs such as caffeine (Hauty \& Payne, 1955), nicotine (Tarriere, Hartemann, \& Niarfeix, 1966) and amphetamines (Mackworth, 1950). With respect to disposition, one of the strongest determinants of performance is the degree to which the subject is motivated to perform the task well (Smith, 1966). This is a particular issue in laboratory-based research, where ecological incentives such as reward or the threat of negative consequences are usually absent. Other individual factors that are often used to predict operational vigilance include sex, age, personality and various indices relating to stress, cognitive resources and psychophysiology (for review, see Reinerman-Jones et al., 2011).

\subsubsection{Contemporary theoretical accounts of vigilance}

Many theories of vigilance were proposed in the years following Mackworth's (1948, 1950) original investigations, each of them offering detailed explanations of the vigilance decrement and general task performance, usually in terms of one principal factor thought to be the primary cause (e.g. inhibition, stimulus expectancy, arousal, motivation: for reviews of these theories, see Frankmann \& Adams, 1962; Parasuraman \& Davies, 1982, pp. 9-24). Modern theories however, as noted recently by Thomson, Besner, and Smilek (2015), generally fall into two broad categories: overload theories and underload theories. Prominent examples of each type of theory are reviewed presently, followed by a review of Thomson et al.'s (2015) resource control theory, which purports to account for the shortcomings of each. 


\section{Overload theories: Resource-depletion}

The principal idea behind overload theories of vigilance is that vigilance tasks are hard work and require effort, and that performance decrements over time are caused by the gradual depletion of a limited pool of information processing resources. The resource depletion hypothesis (Grier et al., 2003; Parasuraman \& Davies, 1977; Warm, Dember, \& Hancock, 1996; Warm et al., 2008) is the most notable variant of this view. It posits that (a) the extent of resource depletion is determined by the specific task demands and the amount of time spent on-task, and (b) the depletion of resources leads to a reduction in the amount of attention that can be allocated to the task, which in turn results in a diminished capacity to detect critical signals (Caggiano \& Parasuraman, 2004). The bulk of evidence supporting this view comes from the numerous studies showing that more demanding tasks (or task conditions) lead to worse overall performance and larger decrements (e.g. Head \& Helton, 2012; Helton \& Russell, 2011, 2013; Helton \& Warm, 2008; MacLean et al., 2009; Parasuraman et al., 2009; Smit, Eling, \& Coenen, 2004; Warm et al., 2008). For example, decrements are ameliorated when the task is made easier by increasing the perceptibility of stimuli (Helton \& Warm, 2008) or by giving warning cues prior to a stimulus (MacLean et al., 2009). Another line of evidence comes from recent studies where reward-based motivation enhanced overall vigilance performance but did not alter vigilance decrements (Esterman et al., 2016; Esterman et al., 2014). This implies that decrements are to some extent caused by the depletion of a limited resource not easily recovered by motivation. As to the nature of the purported resource that is being depleted there has been some debate (e.g. Kurzban, Duckworth, Kable, \& Myers, 2013). It could be that the resource is metabolic in nature (e.g. related to glucose: see Hagger, Wood, Stiff, \& Chatzisarantis, 2010; Kurzban et al., 2013, for discussions on this view), that it is associated with physiological mechanisms involved in circadian rhythms, homeostasis or fatigue (Gunzelmann, Gross, Gluck, \& Dinges, 2009), or that it is more perceptual in its origin, perhaps linked to habituation and adaptation in sensory brain regions (Grill-Spector, Henson, \& Martin, 2006).

\section{Underload theories: Mindlessness and mind-wandering}

Underload theories argue that vigilance tasks are monotonous and understimulating, and that prolonged exposure causes people to gradually withdraw their attention from the perceptual input and therefore become less capable of detecting critical signals. A well known example is the mindlessness hypothesis, which claims that performance decrements emerge when a supervisory attentional system fails to steer attention towards an increasingly monotonous and understimulating task (Manly, Robertson, Galloway, \& 
Hawkins, 1999; Robertson, Manly, Andrade, Baddeley, \& Yiend, 1997). A shortcoming of this account however is that it does not specify what happens to attention when it is withdrawn from the task. Do individuals literally become mindless, or is it simply that their attention is directed elsewhere internally? Noting this lack of specificity, Thomson et al. (2015) suggested that a more accurate and tractable reformulation might be in terms of mind-wandering, rather than mindlessness.

Mind-wandering has been the subject of much investigation outside of the vigilance literature and has a strong theoretical basis (e.g. Mittner et al., 2016; Schooler et al., 2011; Smallwood \& Schooler, 2006, 2015). Specifically, it is thought to be characterised by the decoupling of attention from the external environment and the redirection of attentional resources towards the processing of thoughts, memories and other internal information (Schooler et al., 2011; Smallwood et al., 2011; Smallwood \& Schooler, 2006). The support for mind-wandering as an explanation of the vigilance decrement and related findings comes from self-report studies showing that mind-wandering is negatively related to performance and occurs more frequently as time spent on-task increases (Cunningham, Scerbo, \& Freeman, 2000; McVay \& Kane, 2009; Smallwood, 2003; Smallwood \& Schooler, 2006; Teasdale et al., 1995), and the theoretical standpoint that episodes of mind-wandering consume the same attentional resources that are required for the primary task (Smallwood, 2010; Smallwood \& Schooler, 2006).

\section{Thomson et al.’s (2015) Resource-control theory}

Overload and underload theories of vigilance disagree as to whether vigilance tasks are effortful, and they make different predictions regarding how task demands and task engagement should affect performance. Reviewing the evidence base for these theories, Thomson et al. (2015) found numerous findings that run contrary to the the predictions of each, and concluded therefore that neither offers a complete explanation. As an alternative, they proposed their resource-control theory, which is derived from the resource-depletion account of sustained attention and both the attentional-resource and control-failure accounts of mind-wandering (McVay \& Kane, 2010, 2012; Smallwood $\&$ Schooler, 2006). Specifically, the theory assumes a limited pool of processing resources, but stresses the importance of executive control to ensure that the resources are allocated to the primary task and not wasted in mind-wandering and self-generated thought, for which the individual has an inherent bias. According to this theory, the performance costs associated with the vigilance decrement are caused by a decline in executive control, which leads to a disproportionate amount of resources being devoted to mind-wandering and not enough to the task in hand. Thomson et al. (2015) argue 
that this theory combines aspects of underload and overload theories to provide a more encompassing yet parsimonious explanation of the vigilance decrement and related phenomena, though its specific predictions and suppositions have yet to be tested with extensive empirical investigation.

\subsubsection{Brain mechanisms of vigilant attention}

The advent and availability of biometric technologies have enabled the identification of physiological and neurophysiological correlates of vigilance. It is now known, for instance, that the vigilance decrement is associated with shifts from higher to lower EEG frequencies (Davies \& Krkovic, 1965; Gale, 1977; Makeig \& Inlow, 1993; O’Hanlon \& Beatty, 1977) and reduced amplitude of specific ERP components associated with target discrimination (Davies \& Parasuraman, 1977; Rohrbaugh et al., 1987). Functional imaging and lesion studies have also revealed that one's ability to maintain vigilant attention depends to a large extent on a right-lateralized network of cortical and subcortical brain regions, including the prefrontal cortex, intraparietal sulcus, temperoparietal junction, anterior insula, thalamus, cerebeller vermis and midbrain (for extensive reviews see Fortenbaugh, DeGutis, \& Esterman, 2017; Langner \& Eickhoff, 2013). Another key observation is that vigilance, insofar as it refers to an organisms state of alertness, is closely linked with the noradrenalin (NA) system of the brain, which originates in the brainstem locus coeruleus (LC: Petersen \& Posner, 2012; Posner \& Petersen, 1990). This will be discussed further in the coming section on pupillometry, particularly in Sections 2.5.3 and 2.5.5, which present evidence that nonluminance-mediated changes in pupil size are linked to activity in the LC-NA system and may therefore serve as a useful method of tracking changes in vigilant attention.

\subsubsection{Vigilance in the real world}

Much of the value of research into vigilance lies in its potential to increase our understanding of human performance in operational environments (Howell, 1993; Koelega, 1992; Parasuraman, 1986; Parasuraman \& Davies, 1977, 1982), the need for which is crucial in today's climate of increasing automation (Parasuraman \& Riley, 1997). As Sheridan $(1970,2012)$ has pointed out, the role of workers in human-machine systems is moving away from active control and more towards passive supervision, where action is required only when problems arise. Examples of automated tasks requiring vigilance in this capacity are military surveillance, seaboard navigation, air-traffic control, cockpit monitoring, industrial process control, robotic manufacturing and power plant regulation 
(Arrabito, Abel, \& Lam, 2007; Dorrian, Roach, Fletcher, \& Dawson, 2007; Johnson \& Merullo, 2000; McBride, Merullo, Johnson, Banderet, \& Robinson, 2007; Satchell, 1993; Warm, 1993; Warm \& Jerison, 1984). Vigilance is also central to many tasks with less automation, like long distance driving, agricultural inspection, cytological screening, electrocardiogram monitoring, and airport baggage inspection, (Gill, 1996; Hancock \& Hart, 2002; Hartley, Arnold, Kobryn, \& MacLeod, 1989; Weinger \& Englund, 1990; Wiener, 1984). In all of these situations, failures of vigilant attention can have consequences ranging from the unfavourable to the catastrophic.

Historically, there was much concern expressed in the human factors and experimental psychology literature regarding the value of vigilance research for improving our understanding of operational tasks such as those mentioned above (e.g. Koelega, 1992; Mackie, 1987; Pigeau, Angus, O’Neill, \& Mack, 1995; Sawin \& Scerbo, 1995; Wiener, 1987). For instance, Mackie (1987, p. 725) argued that findings from laboratory experiments lacked generalisability due to their use of "esoteric tasks, limited watch durations, and sterile experimental environments", and Wiener (1987) argued that progress was not being made because of a lack of interest in bridging the gap between the laboratory and the real world. Though such criticisms were perhaps justified in their time, the situation today has improved substantially. There are now an increasing number of experiments which study the performance of both experts and nonexperts in realistic settings or simulated environments (e.g. Dadashi, Stedmon, \& Pridmore, 2013; Donald, Donald, \& Thatcher, 2015; Dorrian et al., 2007; McBride et al., 2007), and there are research-based recommendations for combatting the vigilance decrement in specific situations through intelligent system design (e.g. Arrabito et al., 2007; Donald, 2008) and selection of appropriate personnel (e.g. Reinerman-Jones et al., 2011). With advances in mechanisation and automation, it seems highly likely that vigilance research will continue to play an important part in understanding the human role in rapidly evolving technological environments.

\subsection{Visual search}

Visual search, the act of looking for a specific target among other objects, is a central aspect of human experience. Every day we perform mundane visual search tasks when pursuing short-term goals, like looking for a face in a crowd, a book on a library shelf, or an icon on a computerised display. There are also many socially important tasks in modern society where favourable outcomes depend greatly on efficient visual search, such as satellite image scrutiny, airport baggage screening, and medical image analysis. 
The requirement for visual search itself arises out of our limited capacity for attention: It is simply not possible to process all of the information in our visual environment. We must choose to focus our attention on specific objects, and finding the specific objects requires search. Given the relevance of visual search in a broad range of practical situations and its close relationship with attention, it is not surprising that it has become a topic of great scientific interest. In preparation for the experimental focus of Chapters 4 and 5, this section looks at the general paradigms used to study visual search in the laboratory, influential theories of visual search, the factors that influence performance in visual search tasks both in the laboratory and in the real world, and finally, at some of the most debated issues in the visual search literature.

\subsubsection{The visual search paradigm}

Laboratory-based visual search tasks typically involve an observer looking for one or more specified target stimuli in differently sized sets of nontarget items and indicating with a key-press whether the target is present or absent. Targets are only present on a percentage of trials, typically $50 \%$, and all other trials consist only of nontarget items. Performance is most commonly assessed by studying accuracy and RT measures (Wolfe, 1998b).

When accuracy is the dependent measure of interest, participants are usually exposed to the stimulus for brief periods, within the range of 50-200 ms (e.g. Bergen \& Julesz, 1983a, 1983b; Braun \& Sagi, 1990; Eriksen \& Spencer, 1969; Palmer, 1994; Palmer, Ames, \& Lindsey, 1993; Sagi \& Julesz, 1985; Shiffrin \& Gardner, 1972; Sperling, 1960), and must then search their internal representation of the stimulus to determine whether the target was present or absent. In these paradigms, a mask often follows the stimulus at variable intervals, which is thought to terminate the internal search. Accuracy can be plotted as a function of the stimulus onset asynchrony (SOA) - the time between stimulus and mask - in order to gain insight into the processes involved in search. The general finding is that easier searches can be performed with a high degree of accuracy even with very short SOAs, but that accuracy in more difficult searches requires longer SOAs, and is unlikely to come close to perfect performance (Wolfe, 1998b). Accuracy methods with brief stimulus presentations are useful where the aim is to reduce or eliminate the possibility of voluntary eye movements.

When RT is the primary measure of interest, which is most commonly the case (e.g. Duncan \& Humphreys, 1989; Treisman \& Gelade, 1980; Wolfe et al., 1989), the display typically remains visible until the participant makes a response. RTs for target-present and target-absent trials are then analysed as a function of set size, and 
Literature review

inferences about performance and cognition are made on the basis of the intercepts and slopes of these RT $\times$ set size functions. The principal finding is that slopes are shallow or flat for easier searches but become steeper as the search becomes more difficult (Wolfe, 1998b).

\subsubsection{Theories of visual search}

The visual search paradigm became firmly established as a method of investigating visual attention following the publication of Treisman and Gelade's (1980) influential Feature Integration Theory (FIT), which was based on the findings from a range of visual search experiments. FIT raised many questions about visual attention that could be addressed with further manipulations to the standard experimental paradigm, precipitating a surge of scientific interest and research. This section reviews FIT along with other prominent theories of visual search, each of which is based primarily on the results of accuracy and RT experiments, but also on their authors' understanding of covert attention and knowledge regarding the physiological structure of the visual system.

\section{Feature integration theory}

The Feature Intergration Theory of visual attention (Treisman \& Gelade, 1980) created a lasting legacy with its distinction between parallel and serial search processes. The central proposition was that basic stimulus features such as colour and orientation are at first extracted in a parallel process which operates over the whole of the visual field. So, when a target can be identified by a single feature, like a red ' $\mathrm{O}$ ' among blue ' $\mathrm{O}$ ', $\mathrm{RT}$ is not influenced by the number of nontarget items in the display and the resulting 'parallel' search function is flat. Conversely, when a target is defined by a conjunction of features which are singularly present in the nontarget items in the display, like a red ' $\mathrm{O}$ ' amongst red ' $\mathrm{X}$ 's and blue 'O's, then 'serial' search is required, and RT increases as a function of the number of nontarget items. These two archetypal cases are illustrated in Figure 2.1.

The principal difference between feature and conjunction search, Treisman and Gelade (1980) argued, is that the latter requires the serial allocation of visual attention and the former does not. In conjunction search, focal attention is required to bind the separable visual features into a unitary object which can subsequently be recognised as a target or a nontarget. The time involved with continually relocating the spotlight of covert attention until a decision can be reached regarding the target's presence 


\section{A}

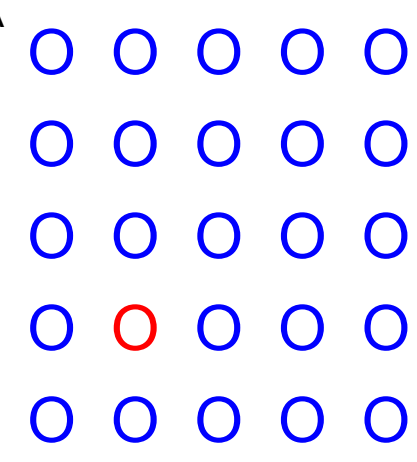

B

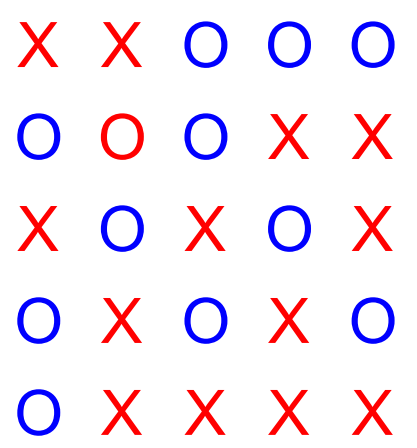

Figure 2.1. The kind of stimuli used by Treisman and Gelade (1980) in their original experiments (find the red ' $\mathrm{O}$ '): (a) feature search, also known as 'parallel' or 'pop-out' search, can be achieved quickly irrespective of set size; whereas (b) conjunction search, also called 'serial' search, takes longer due to the requirement for serial allocation of the spotlight of attention and therefore increases with respect to set size.

or absence gives rise to the steep 'serial' search functions that are associated with conjunction searches. This is the essence of FIT.

A central assumption of FIT is that the visual scene is coded initially along discrete channels, an idea which pairs nicely with neurobiological studies revealing that separate visual properties are selectively processed by specialised neural populations and represented in different regions of cortex (e.g. Maunsell \& Newsome, 1987; Zeki, 1976). The parallels between the assumptions of FIT and the modular nature of the visual system enhanced the appeal of the model, and in the years following its inception, it enjoyed widespread popularity and was taken by many researchers as a novel framework for the psychophysical investigation of early vision. Before long, however, some of the central assumptions of the theory would be challenged by new experimental work. For instance, it was soon clear that the distinction between parallel and serial processing was not so clear-cut and that RT $\times$ set size slopes could vary continuously between the two extremes (e.g. Duncan \& Humphreys, 1989; Wolfe, 1998b). Evidence also emerged that search for targets defined by a conjunction of features could yield flat RT $\times$ set size slopes (e.g. McLeod, Driver, \& Crisp, 1988; Nakayama \& Silverman, 1986), which is at odds with the assumptions of FIT. The theory was refined to accommodate these new experimental advances (Treisman, 1988, 1991, 1993; Treisman \& Sato, 1990; Treisman $\&$ Souther, 1985), but it maintained its two-stage architecture and the central metaphor of a covert attentional spotlight which serially scans the visual scene. 


\section{The Guided Search model}

The Guided Search model (Wolfe et al., 1989) was originally proposed as an alternative to FIT to account for data that was at odds with the parallel-serial dichotomy in general, and more specifically, the proposition that conjunction searches are always serial and self-terminating. In the experiments which formed the basis of the guided search model, Wolfe et al. (1989) observed shallow slopes (relative to what would be predicted by FIT) for colour-form, colour-orientation and colour-size conjunction searches. They also found that slopes for triple conjunction searches of colour-form-size were much shallower than would be predicted by FIT. This lead Wolfe et al. to theorize that the feature maps associated with the parallel stage of processing function together as an attention map - or activation map-which guides the serial deployment of attention by partitioning a stimulus set into nontargets and potential targets. So, when the target is green, serial search will be guided towards green items. The efficiency of the search is determined by the quality of guidance offered by the activation map.

Extensive modelling of visual search behaviour with parameters derived from the original Guided Search experiments has yielded reliable and replicable results (Cave $\&$ Wolfe, 1990), and the model has been continually developed to be brought into alignment with the latest theoretical developments and experimental findings (Wolfe, 1994a, 2006; Wolfe, Cain, Ehinger, \& Drew, 2015; Wolfe et al., 1989; Wolfe \& Gancarz, 1997). In its current state, the Guided Search model uses a single set of parameters to account for a wide-range of search behaviours in laboratory-based tasks. Although the model has been criticised for lacking generalisability to complex real-world visual behaviour, it has inspired work on visual search behaviour for more naturalistic settings (e.g. Võ \& Wolfe, 2012; Wolfe, 1994b) and together with FIT it has served as a critical foundation for the development of advanced computational models of visual cognition (e.g. Itti \& Koch, 2000; Itti, Koch, \& Niebur, 1998).

\section{Attentional engagement theory}

Duncan and Humphreys $(1989,1992)$ proposed a theory of visual search quite different to FIT and Guided Search. They conducted a series of letter-search experiments where they manipulated the orientation, size and eccentricity of target and distractor letters (e.g. participants were required to locate an upright ' $\mathrm{L}$ ' among ' $\mathrm{T}$ 's rotated $90^{\circ}$ clockwise $)^{5}$. Their results suggested to them that search efficiency varies on a continuum, which lead to their rejection of the parallel-serial (efficient-inefficient) dichotomy proposed

\footnotetext{
${ }^{5}$ The essential 'features' in this kind of search are lines of different lengths and orientations, terminators (line endings) and intersections (line crossings).
} 
by FIT. Instead they argued that stimulus similarity determines search efficiency, with a range of search slopes arising from different combinations of target-distractor and distractor-distractor similarity.

Duncan and Humphreys' theory is organised into three distinct stages. The first is a parallel perceptual description stage where a hierarchically structured representation of the visual field is generated in accordance with the kind of grouping laws described by Marr (1982) and Biederman and Ju (1988). Then follows a selection stage where an internal target representation is matched against structural units in the descriptive representation. Finally, structural units with sufficient resemblance to the target representation gain access to VWM, where they are brought into the focus of behaviour and can guide decision and action. The closer the match with a target template, the more likely a structural unit will enter VWM (the converse being true for a distractor template). Unlike FIT and Guided Search, Duncan and Humphreys' theory does not rely on a serial spotlight metaphor of covert visual attention; instead, it postulates the existence of a limited cognitive resource that is allocated to structural units, helping them compete for access into VWM.

\section{Signal detection theory-based approaches}

As well as serving as a useful method for evaluating performance in vigilance tasks (note Section 2.2.1), SDT has also served as the basis for theoretical approaches to visual search (e.g. Eckstein, Thomas, Palmer, \& Shimozaki, 2000; Palmer, Verghese, \& Pavel, 2000; Verghese, 2001). Such theories are in a different class altogether, and differ with respect to the aforementioned principally in that they do not rely on multi-stage architectures (e.g. the parallel-serial dichotomy of FIT and Guided Search). Instead they assume a single stage where all display items are processed in parallel, each evoking noisy internal representations on the basis of their visual properties. The target will score highest on the relevant feature dimension(s), but the effects of neural noise in the visual system mean that overlap in the distributions of internal item representations will occur. The overlap is greater when targets and distractors are similar. Decisions regarding the presence or absence of a target are based on a rule, one of the most popular being the MAX-rule (Hulleman \& Olivers, 2017), where the decision is made on the basis of the single item which scored highest on the relevant feature dimension(s). As the number of distractors increases, so does the probability that one of them will evoke a target-like internal representation, which means that the evidence for a target being present decreases in accordance with an increase in set size. 


\subsubsection{Factors affecting visual search performance in the labora- tory and the real world}

From the vast and burgeoning visual search literature of the last several decades it is possible to identify a range of factors that affect performance. Of course, in visual search tasks which require prolonged and uninterrupted search, then performance could also be affected by any of the factors known to affect vigilant attention, some of which were outlined in Section 2.2.2; but this section focuses specifically on the effects that stimulus properties and other task-related factors specific to visual search have on performance.

\section{Stimulus properties}

One of the clearest findings to emerge from the experimental work that formed the basis of FIT (Treisman \& Gelade, 1980) and Guided Search (Wolfe et al., 1989) is that stimulus features are an important determinant of search performance. Notably, if a target is defined by a single unique feature, like the case in Figure 2.1a, then search is highly efficient. The Guided Search theory proposes that this is because features guide attention towards the relevant parts of the visual field. But what constitutes a feature, and which features guide attention are important questions in their own right. Reviewing these questions, Wolfe and Horowitz (2004) found strong and convincing evidence that colour, motion, orientation and size all guide attention; but also that there were many examples of probable and possible features that facilitate guidance, including but not limited to curvature, steroscopic depth, shading, and line termination. In addition to the low-level visual effects of features, research has shown that search becomes more difficult in accordance with increased similarity between targets and distractors (e.g. Nagy \& Sanchez, 1990; Nothdurft, 2000) and increased heterogeneity of distractors (Duncan \& Humphreys, 1989). Other notable effects of stimulus properties are that targets are harder to detect as they become more abstractly defined (Wolfe \& Horowitz, 2004), as the visual scene becomes more cluttered (Beck, Lohrenz, \& Trafton, 2010; Rosenholtz, Li, \& Nakano, 2007), and as retinal eccentricity of search elements increases (Carrasco, Evert, Chang, \& Katz, 1995; Geisler \& Chou, 1995; Scialfa \& Joffe, 1998).

\section{Target prevalence}

In a visual search task, the proportion of trials where a target is present is referred to as target prevalence. In Section 2.3.1 it was noted that typical laboratory search tasks have 
a target prevalence of $50 \%$, a setting which aims to ensure that participants actually engage with the search task and do not merely guess. However, a substantial amount of research shows that prevalence itself plays a large role in determining performance, with lower prevalence corresponding to higher rates of miss errors and decreased RTs on target-absent trials (Godwin, Menneer, Cave, Thaibsyah, \& Donnelly, 2014; Godwin, Menneer, Riggs, Cave, \& Donnelly, 2014; Wolfe, Brunelli, Rubinstein, \& Horowitz, 2013; Wolfe, Horowitz, \& Kenner, 2005; Wolfe et al., 2007; Wolfe \& Van Wert, 2010). This resonates strongly with the robust findings from the vigilance literature which show that detection rate is reduced when background event rate increases (e.g. Broadbent \& Gregory, 1965; Colquhoun, 1961; Mackworth, 1970) ${ }^{6}$. A common interpretation of the prevalence effect is that individuals respond to low target prevalence by adopting a conservative decision criterion, meaning they are less likely to consider something a target when the a priori probability of its actually being a target is low (Wolfe et al., 2007).

The effects of target prevalence are of prime importance when we consider that socially important search tasks_-like airport baggage and medical image screening — have a very low natural target prevalence. In routine mammography the probability of an image showing signs of cancerous tissue is around $0.3 \%$ (Gur et al., 2004), and in airport baggage inspection, though the probability of finding an item requiring some form of action is less rare ${ }^{7}$, it is still much lower than the $50 \%$ standard used in the laboratory (Rubinstein, 2001; Wolfe et al., 2013).

\section{Number of targets}

Search for a single target is the most common form of laboratory search task, but real-life searches often involve multiple targets with different identities. To return to the examples of radiology and airport baggage screening, a mammogram may contain two separate

\footnotetext{
${ }^{6}$ Low-prevalence visual search tasks are similar to vigilance tasks in that both require search for an infrequent target. However, in visual search, the target generally remains present until a response is made, whereas vigilance tasks are more likely to contain faint and infrequent signals. As Wolfe et al. (2005) point out, this is an important difference, because it means that a fleeting lapse in attention will not lead to an error. Another important difference is that visual search involves looking for a target among concurrent distractors, whereas vigilance tasks usually present stimuli one at a time (Wolfe et al., 2007).

${ }^{7}$ Whilst the probability of finding a genuine threat (e.g. guns, knives, improvised explosive devices) is extremely rare, transportation security officers must also search for prohibited items (e.g. water bottles, certain electronic equipment) and areas of opacity which could be concealing a threat or prohibited item. On top of this, quality control measures such as 'Threat Image Projection' are used to place target items in the X-ray image in order to test the speed and accuracy of operator search performance (Hofer \& Schwaninger, 2005; Rubinstein, 2001; Schwaninger \& Bolfing, 2008). The extent to which Threat Image Projection increases target prevalence is security sensitive information, but it is safe to assume that the overall prevalence of items requiring action remains below the $50 \%$ typically used in laboratories (Wolfe et al., 2013).
} 
abnormalities and an X-ray image of a passengers bag may contain several prohibited items. An established finding in the first case is that the probability of detecting a given abnormality is lower if another abnormality has already been detected (Berbaum et al., 1990; Samuel, Kundel, Nodine, \& Toto, 1995; Tuddenham, 1962), which is known in the radiology literature as 'satisfaction of search' (Wolfe, Palmer, \& Horowitz, 2010). This finding has also been demonstrated in the cognitive psychology literature using simplified displays and nonexpert searchers (e.g. Adamo, Cain, \& Mitroff, 2013; Fleck, Samei, \& Mitroff, 2010; Gorbunova, 2017), although in this context it is sometimes referred to differentially as 'subsequent search misses' (e.g. Adamo et al., 2013; Biggs, 2017; Cain, Adamo, Mitroff, Cosson, \& Dash, 2013; Gorbunova, 2017). A similar finding is that simultaneously searching for two dissimilar targets, which may not be present in the same display, has a negative impact on performance accuracy compared to searching for either target on its own (Godwin, Menneer, Cave, \& Donnelly, 2010; Godwin, Menneer, Cave, Helman, et al., 2010; Menneer, Barret, Phillips, Donnelly, \& Cave, 2007; Menneer, Cave, \& Donnelly, 2009). This has been called the 'dual-target cost', and is especially relevant in the context of airport baggage screening, where operators must search for many different broadly defined items (e.g. Menneer, Donnelly, Godwin, \& Cave, 2010).

Another real-world example of multiple target search that has been studied in the laboratory is foraging (e.g. Cain, Vul, Clark, \& Mitroff, 2012; Ehinger \& Wolfe, 2016; Wolfe, 2013; Zhang, Fougnie, Gong, Alvarez, \& Wolfe, 2015), examples of which are picking berries in a field of berry bushes or apples in an orchard. Assuming that the aim of a forager is to maximise her gain, she will adopt the optimal foraging strategy of picking as many of the best fruits (e.g. the most ripe apples or the biggest berries) in as short a time as possible (Charnov, 1976; Pyke, Pulliam, \& Charnov, 1977; Stephens \& Krebs, 1986). The interesting question here therefore is not when the forager decides to terminate the search, but when she decides that it is time to abandon the current patch and move on to the next. One influential address to this problem, known as Marginal Value Theorem (Charnov, 1976), predicts that the forager will move to the next patch not when they have found all of the targets, but when the average rate of return from the current patch drops below the average for the entire field ${ }^{8}$. If this is correct, given the evolutionary significance of foraging behaviour, it is likely that the tendency is so deeply ingrained as to be a causal factor of errors in modern tasks demanding exhaustive

\footnotetext{
${ }^{8}$ The average rate of return depends on a range of factors, like the speed with which resources can be gathered from a patch, seasonal effects on patch quality, and the time taken to travel between patches (for further information, see Wolfe, 2013).
} 
search, where there can be serious consequences if even a single 'berry' is missed (Wolfe, 2013).

\section{Visual context}

In the laboratory, the most common form of visual search experiment involves participants searching for a simple target in arrays of simple items, configured randomly on a display screen with a blank background. Clearly this is different from real-world scenarios, where the objects of search and their surroundings are generally of greater complexity. As an example, compare searching for the red ' $\mathrm{O}$ ' in Figure 2.1b to the task of searching for an apple in a friend's kitchen. In both tasks, attention may be guided towards the relevant features (i.e. redness / roundness), but in the real-world example it may also be guided by semantic or episodic knowledge regarding the likely location of the target (Evans, Greene, Wolfe, \& Võ, 2011; Wolfe \& Horowitz, 2017). That is, we know that an apple is more likely to be found on a kitchen surface or near a banana than on the floor or near a shoe (semantic guidance); and if we remember the location of the fruit bowl from a prior visit, this knowledge may guide us quickly towards our target (episodic guidance). Though largely overlooked by early theories, knowledge regarding the effects of visual context on search performance and object identification in naturalistic scenes has been available for many years (e.g. Biederman, 1972; Loftus \& Mackworth, 1978; Mackworth \& Morandi, 1967; Palmer, 1975; Yarbus, 1967).

A drawback to studying the effects of visual context on search performance in naturalistic scenes is that 'visual context' is hard to define and control for experimentally (Chun, 2000). Without an efficient way to quantify the semantics of a scene, to define the positional constraints of the objects within it, or to account for differences in learning and the influence of prior knowledge, results can be ambiguous and difficult to interpret. In an attempt to overcome these limitations, Chun and Jiang $(1998,1999)$ devised what they called the contextual cueing paradigm, which closely resembles a traditional visual search task, but has under-the-hood manipulations designed to examine the effects of visual context on search performance. In their original work, Chun and Jiang (1998) asked participants to search for letter 'T's rotated either to the left or the right, hidden amongst rotated letter 'L's, and to respond as quickly as possible by pressing one of two buttons to indicate the direction of rotation. Crucially, a subset of stimuli was repeated across the course of the experiment, with fixed spatial arrangements of the target and distractor elements. Despite being unaware of this repetition and having no explicit memory of target locations, participants identified targets consistently faster on the repeated displays compared to novel displays, suggesting that their performance 
was being facilitated by implicit learning of associations between target locations and spatial contexts. Similar findings have also been reported when visual context is present in the form of motion trajectories (Chun \& Jiang, 1999) and novel 2- and 3-dimensional shape associations (Chua \& Chun, 2003; Chun \& Jiang, 1999).

\subsubsection{Debated issues}

The early theories of visual search offered new and promising ways to study complex visual phenomena in the laboratory but they began a research tradition that generated much debate, often due to dichotomous terminology for describing how attention operates. This section presents an up-to-date view on two of the topics identified by Wolfe (2003) as sources of enduring controversy in the visual search literature.

\section{Preattentive vs. attentive processes}

Guided Search (Wolfe et al., 1989) and related theories (e.g. Itti \& Koch, 2000; Li, 2002; Nothdurft, 2000; Treisman \& Gelade, 1980) proposed that attention is directed towards salient parts of a scene by the activity of feature-selective neurones in the early visual stream. These 'preattentive processes' were thought to provide a rough-cut abstraction of visual input which can assist attention via two main mechanisms: a bottom-up, stimulus-driven mechanism which highlights particularly salient items in the visual field (Li, 2002; Nothdurft, 2000), and a top-down, goal-driven mechanism which is centred around the task demands to which the observer is subject (Hodsoll \& Humphreys, 2001). The notion of preattentive processing goes back at least to Neisser (1967), and in the context of visual search most researchers today would agree that a location in the visual field receives some form of processing prior to its becoming the object of selective attention (e.g. Wolfe, 2003). However, where once there was interest in categorizing visual mechanisms as either preattentive or attentive, now there is discomfort with the rigidity of this approach. As Nakayama and Joseph (1998) pointed out, it is not parsimonious to postulate a new preattentive module every time an experiment reveals a new visual element that assists rapid target detection. Further, many studies in the last 25 years have shown that tasks which were once considered preattentive, such as contrast discrimination and texture segmentation, are themselves affected by attention (for review, see Carrasco, 2011).

An alternative account to the rigid two-stage view was advanced by Di Lollo, Kawahara, Zuvic, and Visser (2001), whose single- and dual-task visual search experiments suggested to them that efficient performance depends not so much on the 
nature of the task, stimuli, or guidance from preattentive feature maps, but rather on the ability of the individual to dynamically reconfigure their visual system, in light of the current task requirements, to deal efficiently with the expected input. This idea resonated strongly with Monsell's (1996) process of task-set reconfiguration, and was succoured by brain imaging studies showing enhanced activity in visual cortex simply to the expectation of impending stimulation (e.g. Gandhi, Heeger, \& Boynton, 1999; Kastner, Pinsk, De Weerd, Desimone, \& Ungerleider, 1999); as well as behavioural studies where novel nontarget stimuli were automatically processed if their physical features were the same as those expected in the imminent display (i.e. the contingent capture phenomenon, see: Bacon \& Egeth, 1994; Folk et al., 1992; Folk, Remington, \& Wright, 1994; Gibson \& Kelsey, 1998). The emergent picture, which is now widely favoured in the literature, is of a dynamic itterative system where information from reentrant processes guides the control of attention around the visual field (Eimer, 2014).

\section{What role does memory play in visual search?}

The extent to which memory processes guide the deployment of attention during visual search has been a subject of controversy (Wolfe, 2003). The origin of the debate can be traced to a study by Klein (1988) which found that participants were slower to respond to a luminance probe when it appeared at a location which previously contained a nontarget item in a visual search task. Klein invoked the concept of inhibition of return (Posner \& Cohen, 1984) as an explanatory mechanism for this effect, intuitively advancing that such a mechanism could facilitate the foraging behaviour of an organism. This idea was called into question as attempts to replicate the findings failed (e.g. Cohen \& Ivry, 1991; Wolfe \& Pokorny, 1990), but was later strengthened by a corroborative study by Klein and MacInnes (1999) which found that participants were slower to foveate a black disc when it appeared suddenly at a location that was close to a recent fixation in a search for Waldo (as in the popular Where's Waldo ${ }^{\mathrm{TM}}$ books).

Although a functional role for memory in visual search such as that proposed by Klein (1988) sounds intuitively like it would be a useful adaptation-it makes little sense to waste time looking in areas we have already checked-others have argued that amnesia may be a more effective strategy for the visual system. Horowitz and Wolfe (1998) tested the role of memory in visual search by comparing performance in a standard visual search task with a static display to that where the display items were randomly relocated every $\sim 100 \mathrm{~ms}$. Performance in these two conditions was comparable despite the inability of participants to remember the locations of the distractors in the random condition. The authors claimed that this finding was not consistent 
with a memory driven search model. The findings were replicated (Horowitz \& Wolfe, 2001) and developed (Horowitz \& Wolfe, 2003), but Wolfe (2003) eventually conceded that the original claim - that 'visual search has no memory' (Horowitz \& Wolfe, 1998, emphasis added) - is too bold to be consistent with the whole of the literature. The present consensus seems to be that inhibition of return can be found in visual search tasks, but most likely on an object-centred rather than a location-centred basis (Müller \& von Mühlenen, 2000; Takeda \& Yagi, 2000; Tipper, Driver, \& Weaver, 1991) and only for a limited number of recently attended items (Danziger, Kingstone, \& Snyder, 1998; Snyder \& Kingstone, 2000). Further indications of a role for VWM in visual search come from studies with eye movements (Gilchrist \& Harvey, 2000; Körner \& Gilchrist, 2007; Kruijne \& Meeter, 2016; Petersen, Kramer, Wang, Irwin, \& McCarley, 2001) and pupillometry (Porter, Troscianko, \& Gilchrist, 2007). These studies are examined in Sections 2.4 and 2.5 , respectively.

\subsection{Eye-tracking and eye movements}

In everyday tasks like reading, tool use, driving, communicating and, of course, visual search, we move our eyes frequently to bring the incoming light from distal objects into alignment with our foveae, where the high density of photoreceptors affords fine resolution of colour and detail. This 'saccade and fixate' strategy is a fundamental principle of how humans and most vertebrates interact with the world (Land, Mennie, \& Rusted, 1999). It is not surprising, therefore, that the scientific study of eye movements has a rich and detailed history (see Wade \& Tatler, 2005, 2012), nor that there has been considerable investment in developing eye tracking technologies for use in eye movement research. Early methods of eye tracking often involved attaching devices directly to the eye (e.g. Ditchburn \& Ginsborg, 1952; Yarbus, 1967), but modern video-based methods can yield accurate and precise measures with procedures that are neither invasive or even obtrusive (Duchowski, 2007). This section looks first at the types of eye movement that can be measured with eye trackers, then it looks at how eye tracking has been used in psychological research, specifically in the study of vigilance and visual search, before finally exploring some of the practical applications of infrared video-based eye trackers. Further details on the methodological principles of video-based eye tracking and the specific methods used in this thesis are provided in Chapter 3. 


\subsubsection{Eye movements and measures}

As mentioned previously, humans use a saccade and fixate strategy to explore their surroundings (Land et al., 1999). Saccades are fast, ballistic movements of the eye that relocate gaze to different parts of the visual field. They can have a broad range of amplitudes, and they have a duration and peak velocity that is proportional to their size (Gilchrist, 2011). Humans make around 3-4 saccades per second, but this changes depending on the activity with which they are engaged (Rayner, 2009). In between saccades are periods of fixation ${ }^{9}$ where the eyes remain relatively still, allowing visual information to be gathered from the environment. In addition to saccades and fixations, human vision is also supported by three other distinct types of eye movement: stabilizing mechanisms (the vestibulo-ocular and optokinetic reflexes), pursuit, and vergence (Land, 2012). These eye movements serve the perceptual functions of maintaining a steady retinal image in various viewing conditions (stabilizing mechanisms and pursuit) and ensuring that the optical axis of each eye intersects at the object of fixation (vergence).

From a cognitive perspective, saccades and fixations are the most interesting eye movements, primarily because they convey information about the moment-to-moment focus of visual attention. However, as noted previously in Section 2.1.1, the spotlight of visual attention can shift without an accompanying movement of gaze (e.g. Posner, 1980), which means that when analysing eye movements over a visual stimulus, one can say that a part of the stimulus was looked at or fixated but not with certainty that it was actually perceived. There is currently no tractable way of gauging the extent to which an object of fixation was perceived other than by looking at the duration of fixation and task performance measures (e.g. accuracy). Fortunately, the separation of covert attention from gaze position appears to occur more in highly constrained experimental conditions than it does in natural vision, which means that in complex goal-driven tasks such as reading, scene viewing and visual search, covert attention is mostly overlapping with gaze position (Rayner, 2009). Beyond the simple analysis of gaze position, it is also possible to derive many other measures from eye tracking data. For instance, eye trackers can give information on the rate, duration, latency, amplitude, accuracy, velocity, direction and curvature of saccades; as well as the frequency and duration of fixations and eye blinks, all of which may be used to make inferences about

\footnotetext{
${ }^{9}$ The term fixation implies stillness, and is therefore technically a misnomer, because the eyes are never perfectly still. Even during periods of so called fixation, the eyes make many tiny movements known as fixational eye movements (i.e. microsaccades, drift and tremor), the function of which has been the subject of much debate (e.g. Engbert \& Kliegl, 2003; Martinez-Conde, Macknik, \& Hubel, 2004; Poletti, Listorti, \& Rucci, 2013; Rucci, Iovin, Poletti, \& Santini, 2007).
} 
the cognitive mechanisms involved in a given task (see below). A comprehensive list of eye tracking measures can be found in Holmqvist et al. (2011).

\subsubsection{Eye tracking in psychological research}

The mainstay use of eye tracking technology is in diagnostic research throughout a range of academic, commercial and industrial domains (see Duchowski, 2002, for a broad overview). In psychological research, eye tracking has been used extensively to study eye movements in many different information-processing tasks. Reading has probably received the most research interest over the years (see Rayner, 1978, 1998, 2009), but there has also been considerable focus on scene perception and visual search (Rayner, 2009). In addition to this, eye tracking has been brought to bear in task domains requiring high levels of expertise such as driving (Land \& Tatler, 2001; Underwood, Chapman, Bowden, \& Crundall, 2002), aviation (Ahlstrom \& Friedman-Berg, 2006; Sarter, Mumaw, \& Wickens, 2007), chess (Charness, Reingold, Pomplun, \& Stampe, 2001; Reingold, Charness, Pomplun, \& Stampe, 2001), medicine (Donovan, Manning, \& Crawford, 2008; Donovan, Manning, Phillips, Higham, \& Crawford, 2005), typing (Inhoff, 1991; Inhoff \& Gordon, 1997), and sport (Land \& Furneaux, 1997; Land \& McLeod, 2000); and it has also been utilised in the study of everyday action tasks like walking (Hollands, Patla, \& Vickers, 2002; Patla \& Vickers, 2003), and making tea and sandwiches (Hayhoe, 2000; Land \& Hayhoe, 2001; Land et al., 1999). An in depth discussion of the findings from such a broad range of studies is beyond the scope of the current thesis. The present section focuses specifically on eye movement research in the two principal topics of interest: vigilance and visual search.

\section{Vigilance}

Vigilance is not a mainstream topic in eye movement research, but the vigilance decrement and associated brain states have been linked to a range of eye movement metrics (e.g. McClelland, Pilcher, \& Moore, 2010; McGregor \& Stern, 1996; Van Orden, Jung, \& Makeig, 2000). Unlike research on other tasks such as reading, scene perception and visual search, where eye tracking is most often used to study how eye movements facilitate effective performance, eye tracking studies of vigilance have tended to focus on how suboptimal performance is reflected in changes in eye movement characteristics over time. For example, a number of studies, involving both real-world simulation tasks and traditional vigilance tasks, have shown that both eye-blink rate and mean eye-blink duration increase in accordance with time-on-task, reflect declines in perfor- 
mance, and correlate with self-reported measures of sleepiness, fatigue and alertness (Brookings, Wilson, \& Swain, 1996; McGregor \& Stern, 1996; McIntire, McKinley, Goodyear, \& McIntire, 2014; Morris, 1996; Stern, Boyer, \& Schroeder, 1994; Van Orden et al., 2000; Wilson, 1990; Wilson \& Skelly, 1987). Similarly, saccaddic rate and saccadic velocity decrease as time spent on-task increases, and this is also associated with declines in performance, sleepiness and fatigue (Becker \& Fuchs, 1985; Bocca \& Denise, 2006; Galley, 1989; McClelland et al., 2010; McGregor \& Stern, 1996; Morris, 1996; Nakayama, Takahashi, \& Shimizu, 2002; Van Orden et al., 2000). Research from the sleep deprivation literature has also linked declines in task performance to various changes in eye movement measures, including those outlined above (e.g. De Gennaro, Ferrara, Urbani, \& Bertini, 2000; Russo et al., 2003; Zils, Sprenger, Heide, Born, \& Gais, 2005).

Taken together, the aforementioned research suggests that the vigilance decrement may arise in part from oculomotor impairments caused by fatigue or sleep deprivation, or from neurophysiological changes, associated with increasing time-on-task, which are reflected in the ocular measures themselves. This has lead some to speculate that eye movement metrics may serve as a useful means of evaluating fitness for duty in operational environments requiring vigilant attention, or perhaps even as part of a real-time monitoring system for predicting performance decrements before they occur (e.g. Kristjansson et al., 2009; McClelland et al., 2010; Van Orden et al., 2000).

\section{Visual search}

Visual search has received a great deal of scientific interest in the last 50 years, but most of the research has been conducted without measuring eye movements and most theories have tended to overlook their significance (Findlay \& Walker, 1999). In their Active Vision account of human visual behaviour, Findlay and Gilchrist (2001, 2003, 2005) criticize theories of visual search and much of the experimental work upon which they are based for placing too much emphasis on covert attentional mechanisms and neglecting one of the more obvious features of human visual behaviour: the use of eye movements to bring objects of interest into alignment with the fovea. Everyday experience readily affirms the importance of eye movements in visual search, as does a wealth of laboratory research (e.g. Findlay, 1997; Findlay \& Walker, 1999; Kowler, Anderson, Dosher, \& Blaser, 1995; Walker, Deubel, Schneider, \& Findlay, 1997); yet the dominant theories overlook this aspect of visual function in favour of much less intuitive ideas, like the passive covert serial scanning mechanism originally proposed by FIT (Treisman \& Gelade, 1980) and maintained in Guided Search (Wolfe et al., 1989). 
This apparent neglect appears to have changed in more recent times, however, as many studies have shown that eye movement measures can yield valuable insights into the moment-to-moment processing of information in visual search tasks (e.g. Najemnik \& Geisler, 2005; Trukenbrod \& Engbert, 2007; Williams \& Pollatsek, 2007; Zelinsky, Rao, Hayhoe, \& Ballard, 1997), and modern theories of visual search are starting to pay more consideration to eye movements (e.g. Eckstein, 2017; Hulleman \& Olivers, 2017; Zelinsky, 2008).

One question to which eye movement studies have contributed substantially is whether visual search has memory. As discussed in Section 2.3.4, this has been a subject of considerable debate. Horowitz and Wolfe (1998) advanced the extreme position that visual search has no memory, but in the years that followed there amassed substantial evidence to the contrary. Much of this was from eye movement studies showing that, whilst observers do sometimes refixate items during search, the pattern of refixations is not consistent with the predictions of a memoryless search model (Beck, Peterson, Boot, Vomela, \& Kramer, 2006; Beck, Peterson, \& Vomela, 2006; Geyer, Von Mühlenen, \& Müller, 2007; Gilchrist \& Harvey, 2000; Körner \& Gilchrist, 2007, 2008; Petersen et al., 2001). For example, refixations tend not to occur for items that were recently fixated (Beck, Peterson, \& Vomela, 2006; Geyer et al., 2007; Körner \& Gilchrist, 2007), and most refixations tend to be directed at targets (Petersen et al., 2001), which implies that search is at least to some extent facilitated by memory for previously visited items.

In addition to progressing the memory debate, eye movement studies of visual search have also helped researchers to understand many other search-related phenomena that were often overlooked in earlier studies, such as the factors that determine where and when people look (e.g. Trukenbrod \& Engbert, 2007; Zelinsky et al., 1997), and the advantages of previewing a search array (e.g. van Zoest, Lleras, Kingstone, \& Enns, 2007; Watson \& Inglis, 2007). Scope and space precludes a full treatment of this research, which is already covered in breadth by Rayner (2009). A final noteworthy point however is that eye movement recordings can be used in conjunction with other physiological measures to gain deeper insight into search processes. Examples of this include the fixation-related potential (e.g. Kamienkowski, Ison, Quiroga, \& Sigman, 2012) and fixation-aligned pupillary response averaging (Klingner, 2010a, discussed further in Section 2.5.5 and Chapter 5) techniques, where the physiological responses are time-locked to fixations on display items. In this type of application, eye movement measures serve an auxiliary purpose, enhancing the spatio-temporal resolution of other physiological measures. 


\subsubsection{Interactive applications of video-based eye tracking}

As mentioned briefly in the previous chapter, the applications of eye tracking technology can be broadly classified as being diagnostic or interactive in nature (see Duchowski, 2002). Research applications are typically diagnostic in that they involve the post hoc, offline analysis of data collected during an experiment. Interactive applications of eye tracking are different in that they involve using the eye tracker as a real-time input device into a computerised system. This section looks briefly at the interactive use of eye tracking in selective systems and gaze-contingent displays.

\section{Selective systems}

In eye tracking-based selective systems, eye gaze is used to select on-screen information in the same way that a mouse would be used. Typical examples include using gaze to select on-screen buttons or menu options (Jacob, 1991) and to operate virtual keyboards (i.e. 'eye typing': see Majaranta \& Räihä, 2002). Such applications have proven extremely useful as a communication modality for physically handicapped users, but in routine circumstances they are usually suboptimal to alternatives (e.g. mouse control) due to a lack of spatial precision and complications involved with selecting on-screen resources (e.g. the 'Midas touch' problem: see Duchowski, Medlin, Gramopadhye, Melloy, \& Nair, 2001). Another innovative use of gaze was given by Vertegaal (1999), who devised a system to facilitate communication in multi-party teleconferencing situations. In this particular example, eye gaze serves to provide on-screen indications of who is talking to who, as well as which parts of a shared document are being looked at by other system users.

\section{Gaze-contingent displays}

In addition to serving as a means for interaction with computers, eye gaze can also be used for gaze-contingent display rendering. This is common in reading and (to a lesser extent) visual search research, where foveal masking and moving window techniques are used to obscure or degrade parts of a stimulus based on gaze position (Rayner, 1998). Outside of the research domain, gaze-contingent methods have been used extensively to enable the intelligent rendering of displays such that high-resolution information is centred at the user's locus of gaze, and peripheral information is reduced or degraded. The purpose of this technique is generally to ease the load on transmission bandwidth and computational resources in demanding visual applications, such as driving and flight simulation, or virtual reality (Reingold et al., 2003). 


\subsection{Pupillometry}

Pupillometry, the measurement of pupil size, is a technique now used widely in psychological research for gaining insight into cognitive function. It became popular in the 1960s following the influential work of Hess and Polt (1960), who linked pupil dilation to the interest value of pictorial stimuli (see below), but its use diminished gradually as neuroscience and other peripheral physiological techniques came to the fore. When video-oculography became widespread in the late 90s, however, researchers gained access to high-resolution information on pupil size simply as a 'by-product' of collecting eye tracking data, which lead to a resurgence of interest and an increasing amount of scientific publications.

Pupil size measures are of central importance to the experimental work in this thesis, so it is essential to learn more about how they have been used previously. This section provides a broad overview of pupillometry research that has been conducted to date, focusing on the cognitive factors that are known to affect pupil size, the neural mechanisms of pupil control, practical applications of pupillometry, and finally, in line with the aims and scope of the current thesis, pupillometric studies of vigilance and visual search. Issues concerning the collection, processing and analysis of pupil size data are reviewed in the following chapter.

\subsubsection{Cognitive modulation of the pupillary light response (PLR)}

The PLR is the well known dilation and constriction of the pupil which occurs following changes in light levels incident on the eye. It is an autonomous bodily function which serves the primary purpose of fine tuning visual acuity by mediating the amount and trajectory of light that strikes the retina (Campbell \& Gregory, 1960; Hirata, Yamaji, Sakai, \& Usui, 2003; Laughlin, 1992; Woodhouse, 1975; Woodhouse \& Campbell, 1975). Although traditionally thought of as being purely reflexive in its nature, converging evidence from the last decade has revealed that the PLR itself is subject to modulation by cognitive factors. One particular finding is that the pupil reacts to subjective perceptions of lightness rather than simply to the physical light that enters the eye. Under conditions of binocular rivalry, where visual consciousness alternates between different stimuli presented to each eye, changes in pupil size reflect the luminance of the stimulus that reaches conscious awareness (Fahle et al., 2011; Naber et al., 2011). These findings echo the results of earlier studies which showed that light flashes elicit more constriction when presented to a dominant eye as compared to when they are presented to the same 
eye under conditions of perceptual suppression (Bárány \& Halldén, 1948; Lorber, Zuber, $\&$ Stark, 1965).

Physically present stimuli are not the only source of cognitive modulation of the PLR. Recent reports have demonstrated that the mere suggestion of a light source being present can lead to pupillary constriction. For instance, studies by Binda, Pereverzeva, and Murray (2013a) and Naber and Nakayama (2013) found greater pupil constriction to pictures of the sun as compared with pictures of identical luminance and closely matched low-level image properties. In a similar vein to this, Laeng et al. (2012) showed that the PLR can be triggered by illusions of brightness, and Laeng and Sulutvedt (2014) even found that simply asking participants to imagine familiar scenarios that are intrinsically dark or bright (e.g. a 'dark room' or a 'sunny sky') causes the pupil to dilate and constrict, respectively. Visually presented and spoken words conveying a sense of brightness and darkness also seem to invoke the PLR (Mathôt, Grainger, \& Strijkers, 2017).

Finally, another class of noteworthy observations are those to do with the effects of selective attention on the PLR. When participants maintain central fixation on displays containing bright and dark areas in the periphery, their pupils will constrict if they attend covertly (i.e. without eye movements) to the bright area and dilate if they attend covertly to the dark area (Binda \& Murray, 2015; Binda et al., 2013a; Mathôt, van der Linden, Grainger, \& Vitu, 2013). Naber, Alvarez, and Nakayama (2013) demonstrated that this phenomenon can be utilised as a means of inferring the locus of covert spatial attention to one of multiple peripherally presented images flickering from bright to dark at different frequencies, ranging from 0.3-3.4 Hz. Research has also shown that the PLR follows feature-based attention to overlapping dark and bright surfaces (Binda, Pereverzeva, \& Murray, 2014) and prepares for the luminance of a saccade target location before the eyes actually move (Mathôt, van der Linden, Jonathan, \& Vitu, 2014). Taken together, these findings strongly suggest that the PLR is not simply a low-level reflex, but that it is affected by higher level cognitive processes and has strong links with the visual attention system of the brain.

\subsubsection{Cognitive factors linked to the psychosensory pupil response}

We know today that every vivid mental event, every physical effort, every impulse of will, each activation of attention, and especially each affect causes pupillary dilation just like every sensory stimulus that flows to the brain from the periphery 
-Oswald Bumke (1910) ${ }^{10}$

Man may either blush or turn pale when emotionally agitated, but his pupils always dilate

—Irene Loewenfeld (1958)

The findings outlined above in Section 2.5.1 show how the PLR is modulated by cognitive factors, but the majority of cognitive pupillometry studies are concerned principally with the psychosensory pupil response (PPR), which is much smaller in magnitude than the PLR and occurs in the absence of luminance changes. These cognitive effects on pupil size have already been the subject of many extensive reviews (see Andreassi, 2000; Beatty, 1982b; Beatty \& Lucero-Wagoner, 2000; Einhäuser, 2017; Goldwater, 1972; Laeng et al., 2012; Sirois \& Brisson, 2014). What follows is a broad overview of the literature in this area, focusing on some of the key cognitive factors that have been linked to the PPR. Findings from well known studies important for understanding the literature on the whole, as well as those from lesser known studies important for the context of the current thesis, are discussed.

\section{Arousal and interest}

In their seminal work on cognitive pupillometry, Hess and Polt (1960) discovered that the pupils of heterosexual males and females dilated more when they viewed nude pictures of the opposing sex compared to when they viewed pictures of the same sex; that the pupils of females dilated more to pictures of babies and pictures of mothers holding babies; and that the pupils of neither males nor females dilated nearly as much when viewing pictures of landscapes. These findings were interpreted in terms of a sexual dichotomy with respect to the interest value of the stimuli, because pupil dilation was greatest when individuals viewed pictures that were logically of more interest to their sex and sexual orientation. A similar picture viewing experiment by Hess et al. (1965) yielded comparable results for heterosexual and homosexual males, with pupil dilation to nudes scaling in accordance with sexual orientation. Returning to these findings years later however, Aboyoun and Dabbs (1998) measured pupil dilation of males and females in response to clothed and unclothed pictures of both sexes and found that dilation was always greater for nudes, irrespective of the sex of the participant, which lead them to question whether the pupil response was mediated by emotional or sexual arousal, as is often assumed, or simply the novelty of the stimuli. A problem

\footnotetext{
${ }^{10}$ Translated in Loewenfeld (1993, pp. 667)
} 
in pinpointing these effects to arousal is that arousal itself is a broad and unspecific aspect of physiology, with connotations ranging from the sexual and emotional through to wakefulness, general task engagement and vigilance. Whilst other studies have found that the pupil dilates to emotional stimuli, such as emotionally charged rather than neutral words (e.g. Bradley, Miccoli, Escrig, \& Lang, 2008), the difficult task is to identify the type of arousal and dissociate the effects of arousal per se from other cognitive factors that cause pupil dilation.

\section{Cognitive load}

Interest in cognitive pupillometry was born out of studies looking at pupil responses to potentially arousing stimuli, but the emphasis soon shifted to information processing. In particular, a substantial amount of research has focused on how pupil size changes with respect to cognitive load. Some of the earliest studies in this area used mental arithmetic tasks to induce cognitive load, showing that the pupil dilates as participants attempt to solve a problem and that the degree of dilation scales with the difficulty of the problem (e.g. Boersma, Wilton, Barham, \& Muir, 1970; Hess \& Polt, 1964). Other studies using different methods have shown that pupil size increases with the number of digits stored in WM (Kahneman \& Beatty, 1966) and in accordance with the difficulty of pitch discrimination tasks (Kahneman \& Beatty, 1967) and letter matching tasks (Beatty \& Wagoner, 1978). It was on the basis of such evidence that Kahneman (1973) chose pupil dilation as the preferred index of mental processing load for his effort-based theory of attention, arguing that its sensitivity to both between-task and within-task variations in processing demands makes it highly suitable. Later research by Ahern and Beatty $(1979,1981)$ also showed that the magnitude of pupil responses to set cognitive tasks differed between groups of participants with high and low psychometric intelligence ${ }^{11}$, which is further evidence for the suitability of pupillometry as an index of cognitive load.

\section{Memory}

Kahneman and Beatty's (1966) general observation that pupil size increases with WM load spurred further investigations into the effects of specific memory processes on the cognitive pupil response. One early study by Magliero (1983) found that pupil size depends on the spacing between repetitions of identical or related words during the encoding phase of an auditory memory experiment, with larger pupils at longer

\footnotetext{
${ }^{11}$ The high intelligence group showed less dilation than the low intelligence group, presumably reflecting the individual differences in cognitive effort required for the tasks.
} 
intervals and smaller pupils at shorter intervals. In a more recent example, Võ et al. (2008) reported a 'pupil old / new effect', whereby words learnt in the encoding phase of a recognition memory experiment elicited larger pupil responses during retrievel than words that were not learnt. Subsequent studies by Heaver and Hutton (2011) and Otero, Weekes, and Hutton (2011) replicated this finding, providing further indication that the pupil response during recognition judgements may reflect the strength of the memory trace from the encoding phase. Whether pupil size at the time of encoding predicts success at recall however is presently unclear. Whilst there is some evidence to this effect (e.g. Kafkas \& Montaldi, 2011; Naber, Frässle, Rutishauser, \& Einhäuser, 2013; Papesh, Goldinger, \& Hout, 2012), there is a lack of agreement as to which metric of pupil size change is associated with better recall (e.g. dilation vs. less dilation vs. constriction). Goldinger and Papesh (2012) provide a good review of the literature on memory studies using pupillometry.

\section{Decision making}

Knowledge regarding a link between pupil size and decision making goes back at least as far as a study by Simpson and Hale (1969), where participants in two groups were asked to make simple lever movements. One group had to decide which direction to move the leaver whereas the other received instruction, and the decision group showed significantly greater pupil dilation than the instruction group. In more recent years, the effects of decision making on the pupil have been subject to fine-grained analysis. One particularly informative study showed how pupil dilation not only accompanies decisions, but betrays the timing of them, even when the button-press response signalling the decision is delayed (Einhäuser et al., 2010). Other studies have shown that pupil dilation predicts gambling decision errors (Preuschoff et al., 2011), provides insight into latent decision processes (Cavanagh, Wiecki, Kochar, \& Frank, 2014) and reveals upcoming choice and individual bias (de Gee, Knapen, \& Donner, 2014), but there is a lack of agreement in this literature as to whether the observed pupil effects reflect the cognitive processes that precipitate decisions, the adaptive shifts in brain state that are provoked by changing task demands, or the final choice which terminates the decision process. It is quite likely that all of these factors have some influence on pupil size, and that the variable interpretations reflect the substantial differences between the kind of decisions made in each of the tasks. 


\section{Attentional orienting and salience}

Transient pupil dilation has long been associated with the orienting response (Lynn, 1966; Pavlov, 1927; Sokolov, 1963), but only recently has this been studied systematically in humans. Wang and Munoz (2014) presented participants with brief peripheral luminance contrast stimuli (gabor patches) and found that they evoked transient pupil responses of a strength and magnitude which scaled with the contrast-based salience of the stimulus. The characteristics of these pupil responses in humans were comparable to those evoked in monkeys by similarly salient stimuli (Wang, Boehnke, Itti, \& Munoz, 2014), and also through microstimulation of their superior colliclus (SC: Wang et al., 2014; Wang, Boehnke, White, \& Munoz, 2012), a brain structure which is strongly implicated in attentional shifts and the orienting response (Boehnke \& Munoz, 2008, also, see below in Section 2.5.3). These findings, together with the numerous studies that have linked stimulus saliency to shifts of gaze and attention (for reviews, see Carrasco, 2011; Kowler, 2011), suggest that transient pupil dilation may be a component in the orienting response to contrast-based stimuli. It has been argued that the function of this purported component of the orienting response is to enhance perceptual sensitivity to the eliciting stimulus (e.g. Lynn, 1966; Wang et al., 2012), but empirical evidence to support this is lacking (Nieuwenhuis, de Geus, \& Aston-Jones, 2010). It should be noted that these effects of attentional orienting are qualitatively different from the PLR-modulating effects of selective covert attention to bright and dark surfaces, mentioned in Section 2.5.1, which are manifest through sustained enhancement of cortical processing of stimuli that would normally cause the pupil to change in size.

\section{Target detection without an overt motor response}

The pupillometry literature is replete with studies where pupil dilation was observed around the time of a motor response signalling some aspect of task fulfilment, often the detection of a target stimulus or event (e.g. de Gee et al., 2014; Einhäuser et al., 2010; Einhäuser et al., 2008; Fahle et al., 2011; Hupé et al., 2009; Kloosterman et al., 2015; Porter et al., 2007; Privitera, Carney, Klein, \& Aguilar, 2014; Privitera et al., 2010; Richer \& Beatty, 1985; Richer, Silverman, \& Beatty, 1983; Smallwood et al., 2011). As noted by Pierre (1973) and later by Hupé et al. (2009), this response likely reflects the additive effects of various component processes, including detection, the decision to respond, and the preparation and execution of the motor command itself. Pinpointing the effects of target detection on the pupil response is therefore challenging in standard experimental paradigms, because detection is almost always indicated with a speeded button-press response. Recently however, some studies have circumvented this issue 
Literature review

by removing the detection response or delaying it until a later point. Privitera et al. (2010) conducted two experiments where participants had to report the presence of targets during rapid serial visual presentation (RSVP). In both experiments, participants signalled target detection with a button-press, but then some of the participants took part in a rerun where they simply had to count the targets or keep looking for targets. Buttonpress detections in the first runs evoked large pupil responses of the kind observed frequently throughout the literature, but simple detections without button-presses (as compared to misses in the button-press conditions) also evoked significant though attenuated pupil responses. Similar effects have also been reported when participants made delayed responses to targets in attentional blink and visual search tasks (Klingner, 2010a; Wierda et al., 2012). Although far from decisive, this evidence suggests that pupil dilation in the absence of an overt behavioural response may serve as a useful index of target detection.

\subsubsection{Neural mechanisms of pupil control}

The aperture of the pupil is determined by the tone of the sphincter and dilator pupillae, the two opponent smooth muscles of the iris. The sphincter is innervated by the parasympathetic system and receives cholinergic input from nerve fibres originating in the Edinger-Westphal (EW) nucleus, which itself is connected to the retina via the pretectal nucleus of the midbrain. Pupil constriction following a light stimulus is effected primarily by excitatory activity in this pathway (Clarke \& Ikeda, 1985; Clarke, Zhang, \& Gamlin, 2003). The dilator on the other hand is under sympathetic control, receiving excitatory input via noradrenergic (NA) projections from the locus coeruleus (LC: Loewenfeld, 1993). The LC also has inhibitory connections to the EW nucleus, which means that pupil dilation, insofar as it is mediated by NA-release in the LC (see below), occurs directly as a result of excitation in the sympathetic pathway to the dilator muscle and indirectly from inhibition of the parasympathetic pathway (Samuels $\&$ Szabadi, 2008a, 2008b). Dissociating these effects (i.e. less constriction from more dilation) is possible through pharmacological intervention (e.g. see Hou, Freeman, Langley, Szabadi, \& Bradshaw, 2005; Koss, 1986; Phillips, Szabadi, \& Bradshaw, 2000), but is not easily achieved with standard pupillometry (although see Steinhauer, Siegle, Condray, \& Pless, 2004). 


\section{Pupil size and the LC-NA system}

The LC is a small brainstem nucleus comprising the largest collection of noradrenergic neurons in the central nervous system (Astafiev, Snyder, Shulman, \& Corbetta, 2010). Through a widespread network of afferent projections, the LC innervates the cortex with the neurotransmitter NA, which is known to play a crucial role-together with other neurotransmitters such as dopamine, serotonin and acetylcholine-in the modulation of arousal, cognition and autonomic function (Berridge, 2008; Berridge, Schmeichel, \& España, 2012; Berridge \& Waterhouse, 2003; Mather \& Harley, 2016; Samuels \& Szabadi, 2008a, 2008b; Sara, 2009; Sara \& Bouret, 2012). Specific theories of LC-NA function (e.g. Aston-Jones \& Cohen, 2005; Bouret \& Sara, 2005; Mittner et al., 2016; Yu \& Dayan, 2005) have recognized two distinct modes of LC activity which correspond to different behavioural states. The phasic mode is characterised by short bursts of activation and supports task engagement, whereas the tonic mode is characterised by sustained increase in baseline activation and supports disengagement. In their adaptive gain theory of LC-NA function, Aston-Jones and Cohen (2005) suggest that transitions between these modes of activity serve to optimise the trade-off between exploitation (phasic) and exploration (tonic) of the environment, helping an organism to adapt its behaviour in order to maximise utility in both the short and long term.

The usefulness of pupillometry for making inferences about cognitive function rests to a large extent on the functional association between nonluminance-mediated changes in pupil size and activity in the LC-NA system. The most direct and striking evidence for this comes from neural recording and microstimulation studies in monkey which show a strong temporal coupling between LC activity and changes in pupil size (Joshi, Li, Kalwani, \& Gold, 2016; Rajkowski, Kubiak, \& Aston-Jones, 1993; Varazzani, San-Galli, Gilardeau, \& Bouret, 2015). Further evidence of a less-direct nature comes from a range of human studies which combined pupil size measurements with pharmacological manipulations, functional imaging, and experimental manipulation of common factors known to drive changes in pupil size and LC activity (Alnaes et al., 2014; Aston-Jones \& Cohen, 2005; Beatty, 1982a, 1982b; de Gee et al., 2017; Einhäuser et al., 2008; Gilzenrat et al., 2010; Hou et al., 2005; Jepma \& Nieuwenhuis, 2011; Morad, Lemberg, Yofe, \& Dagan, 2000; Murphy, O'Connell, O’Sullivan, Robertson, \& Balsters, 2014; Murphy, Robertson, Balsters, \& O'Connell, 2011; Phillips et al., 2000; Richer \& Beatty, 1987; Urai, Braun, \& Donner, 2017). Taken together, this evidence strongly implies that fluctuations in pupil size under conditions of constant luminance are closely related to LC activation; but as Costa and Rudebeck (2016) warn in their comments on Joshi et al.'s (2016) findings, the precise neural mechanisms 
of this relationship are presently unclear. It is quite possible that the pupil-LC-NA relationship is mediated by a third variable, as of yet not understood.

\section{Pupil size and the superior colliculus (SC)}

Although cognitive effects on pupil size have most frequently been attributed to the activity of the LC-NA system, evidence from recent years suggests that activity in the $\mathrm{SC}$ is also an important determinant. The $\mathrm{SC}$ is a phylogenetically ancient midbrain structure which plays a causal role in the control of spatial attention and eye movements (Gandhi \& Katnani, 2011; Krauzlis, Lovejoy, \& Zénon, 2013; White \& Munoz, 2011), and is thought to encode salience and behavioural relevance of stimuli in service to various components of the orienting response (Boehnke \& Munoz, 2008; Corneil \& Munoz, 2014; Fecteau \& Munoz, 2006; Knudsen, 2007; McPeek \& Keller, 2004; Mysore \& Knudsen, 2013; Sparks, 1986). Evidence linking transient pupil dilation to the orienting response and SC activity in humans and other animals was discussed previously in Section 2.5.2. Most striking are the findings from studies in nonhuman animals showing that microstimulation (subthreshold for saccade generation) of SC (or its homologue) triggers transient pupil dilation (e.g. Netser, Ohayon, \& Yoram, 2010; Wang et al., 2014; Wang et al., 2012), and that salient stimuli evoke both transient pupil dilation and SC activation which scales with stimulus salience (Netser, Dutta, \& Gutfreund, 2014; Wang \& Munoz, 2014). Collectively, these findings implicate the SC in cognitive modulation of pupil size, but as with the pupil-LC link, the neural mechanisms are not fully understood. A detailed review of the full range of the evidence for cognitive modulation of pupil size by activity in the SC is given by Wang and Munoz (2015), and what is known about the underlying neural circuitry by Corneil and Munoz (2014). An important point to note is that the SC and LC could subserve complementary functions in the cognitive modulation of pupil size, and need not be thought of as being mutually exclusive. Dissociating their relative contributions will no doubt be the aim of future studies combining pupillometric and neurophysiological methods.

\subsubsection{Practical applications of cognitive pupillometry}

The reliable and observable PLR has long served physicians and clinicians as a practical method of assessing retinal and brainstem function. The cognitive pupillary response on the other hand is much smaller in magnitude, requires specialist equipment in order to obtain reliable and meaningful measurements, and lacks specificity regarding the underlying neurocognitive mechanisms. Historically this has constrained the practical 
utility of cognitive pupillometry, but this has changed with the advent, development and widespread availability of video-based eye tracking equipment. The present section sheds light on some of the progress that has been made by focusing in turn on a selection of the practical applications of cognitive pupillometry that have become the focus of research.

\section{Lie detection}

Knowledge of the connection between pupil size and cognitive factors such as effort, arousal and decision making has prompted some to investigate the potential for using pupillometry as a means of detecting lies and deception. In an early example, Berrien and Huntington (1943) found increased pupil dilation in 'guilty' participants from a mock crime experiment compared to those who were 'innocent'. Subsequent studies have also shown that participants' pupils dilate more when they harbour guilty knowledge as to when they do not (Lubow, 1996), and when they provide deceptive rather than honest information in a range of contexts (Dionisio, Granholm, Hillix, \& Perrine, 2001; Heilveil, 1976; Janisse \& Bradley, 1980; Wang, Spezio, \& Camerer, 2010). Together these findings suggest that pupillometry could be useful as a measure of deception in scenarios where the emphasis is on additive effects over a series of trials (e.g. see Wang et al., 2010), but the lack of specificity and sensitivity of pupil responses to individual acts of deception means that a pupillometric lie detection tool fit for use in court or interrogation is untenable. Further, as with the traditional polygraph method of lie detection, where accuracy can be reduced by countermeasures such as counting backwards from 7 or biting one's tongue during control questions (Honts, Devitt, Winbush, \& Kircher, 1996), an individual could easily fool a pupillometric lie-detection system by doing some mental multiplication or thinking of a bright or dark room.

\section{Assessing sleepiness}

As well as responding reflexively to light and psychosensory stimulation, the pupils can also exhibit synchronous and rhythmical patterns of dilation and constriction, which are known as pupillary hippus, or sometimes, pupillary unrest. This behaviour has been linked by many studies to fatigue and sleepiness in the past (e.g. Crawford, 1936; Lowenstein, Feinberg, \& Loewenfeld, 1963; Lowenstein \& Loewenfeld, 1964; Yoss, Moyer, \& Hollenhorst, 1970a, 1970b), and in more recent years, it has become the focus of attempts to infer these brain states from pupil data. McLaren, Erie, and Brubaker (1992) developed a method of computerized analysis whose assessment of alertness 
from pupillograms correlated highly with the subjective assessments of 3 experienced physicians; and many other studies have found good levels of agreement between indices of pupillary unrest and self-reported sleepiness (e.g. Lüdtke, Wilhelm, Adler, Schaeffel, \& Wilhelm, 1998; Morad et al., 2000; Wilhelm et al., 2001; Wilhelm, Lüdtke, \& Wilhelm, 1998). All in all, this is a successful application of pupillometry, and purpose built systems for gauging sleepiness from measurements of pupil size are now commercially available (e.g. the Pupillographic Sleepiness Test by AMTech, Weinheim, Germany).

\section{Real-time assessment of cognitive workload}

The association between pupil size and cognitive load has found practical utility in real-time monitoring situations. One method, the Index of Cognitive Activity ${ }^{\mathrm{TM}}$ (Marshall, 2002), is a patented pupillometric technique for acquiring objective measures of cognitive workload in real-time. Its utility lies in its purported efficiency at measuring cognitive pupil reflexes whilst factoring out those caused by changes in retinal illumination. Due to its trademark status, there is little readily available information about how the Index of Cognitive Activity ${ }^{\mathrm{TM}}$ is calculated, but more general information about the technique and its proposed uses can currently be obtained at the following web address: http://www.eyetracking.com/Software/Cognitive-Workload. Another good example of real-time monitoring with cognitive pupillometry was given by Bhavsar, Srinivasan, and Srinivasan (2016), who examined the performance of 44 participants as they played the role of 'control room operator' in a highly realistic ethanol process simulation task. During the task, participants had to recognise and respond to various plant abnormalities, and measurements of pupil size in the abnormal situations showed distinct signatures of their abilities to successfully manage the problem.

\section{Communicating with 'locked-in' patients}

Stoll et al. (2013) demonstrated how patients with 'locked-in' syndrome, who have suffered complete loss of motor control, can signal 'yes' and 'no' responses by using mental arithmetic as a tool to modulate their pupil size. Patients were first asked a question, to which the correct answer was already known, and were then presented with subsequent displays showing mathematical problems corresponding to the answers 'yes' and 'no'. Patients were instructed to attempt to solve the problem which corresponded to the appropriate answer to the question, and based on the timing of pupil responses Stoll et al. (2013) were able to decode the correct answer with above-chance accuracy for most patients and up to $90 \%$ accuracy for one particular individual. Although 
limited to a throughput of around 2 'yes' / 'no' questions per minute, this method requires no movement and does not depend on any form of training, making it useful for establishing first contact (i.e. testing awareness) with patients in a vegetative state.

\section{Voluntary manipulation of pupil size as an active HCI input channel}

In the aforementioned example, voluntary control over pupil size via cognitive exertion enabled interpersonal communication in a contrived situation. Mounting evidence also suggests that voluntary manipulation of pupil size through cognitive exertion and various other strategies may serve a useful purpose as a generalised input mechanism into HCI systems. Ekman, Poikola, Mäkäräinen, Takala, and Hämäläinen (2008) were among the first to demonstrate this, with a study where participants attempted to intentionally increase or decrease their pupil size using strategies of physical regulation (e.g. relaxation, self-induced pain), cognitive processing (e.g. reversing words in their mind, performing calculations) and affective control (e.g. positive and negative imaginations). Live feedback was provided during testing sessions in the form of a black circle on a green background which expanded and contracted to show modulation from baseline. The results clearly showed that participants could alter their pupil size by up to $20 \%$ using the various strategies, with effects emerging around $1.5 \mathrm{~s}$ after trial onset and stabilizing after around $3 \mathrm{~s}$. Subsequent similar work by Ehlers and colleagues (Ehlers, Bubalo, Loose, \& Huckauf, 2015; Ehlers, Georgi, \& Huckauf, 2014; Ehlers, Strauch, Georgi, \& Huckauf, 2016) has supported these initial reports, but also revealed that the capacity for self-regulation of pupil size with live feedback varies substantially between individuals and strategies, does not appear to improve with training over a 4-week period, and is only effective for increasing rather than reducing pupil size. Therefore voluntary pupil size changes may in principle serve as a viable input modality into HCI systems, but further work is needed to establish the true potential of this method.

\section{The mind-writing pupil}

In Section 2.5.1 it was noted that the PLR is affected by luminance levels at the locus of covert spatial attention, and that this enabled Naber, Alvarez, and Nakayama (2013) to infer which of multiple objects flickering from bright to dark at different frequencies in the periphery was being covertly attended to by a participant. Building on these findings, Mathôt, Melmi, van der Linden, and Van der Stigchel $(2015,2016)$ were able to design an innovative pupil-computer-interface keyboard which allowed individuals to spell words and write short sentences without ever actually looking at the required letters (hence they named it 'the mind-writing pupil'). In their method, participants 
fixate a central point on the screen and attend covertly to their choice of multiple letters, which are situated in the periphery within circles that oscillate between bright and dark. Importantly, half of the circles oscillate in counterphase to the other half, which means the oscillations in pupil size-which reflects the brightness changes of the attended area — can be used to progressively rule out half of all possible choices until only the target letter remains ${ }^{12}$. Although spelling with this method was laborious, with each character of functional text taking on average $75.2 \mathrm{~s}$ to generate, selection accuracy was around $90 \%$. This makes the mind-writing pupil a potentially viable solution to some practical problems where time is not of the essence. The authors suggested it could be used as a means of communicating with 'locked-in' patients, to input passwords in an ultra secure fashion, and to train sustained attention.

\section{Pupil-assisted target selection (PATS)}

Recent work suggests that the short-latency pupil dynamics which accompany target detection without an overt motor response (see Section 2.5.2) could help to facilitate input commands in eyes-only HCI. Strauch, Greiter, and Huckauf (2017a, 2017b) are in the process of developing a method of pupil-assisted target selection (PATS), which integrates short-latency pupil size changes and fixation dwell time information to infer user choice. With PATS, users signal their intention to select on-screen objects by fixating them, and selection is achieved when certain predetermined criteria regarding dwell time and pupil size changes are fulfilled. An initial demonstration (Strauch et al., 2017a) was given with a search and selection task, where participants had to select a target shape from several shapes positioned in a circle. For this task, selection with PATS was affirmed when fixation dwell time exceeded $1000 \mathrm{~ms}$ or when pupil size surpassed a predetermined threshold (calculated individually for each participant), whichever occurred first. Selection times with PATS were consistently faster than they were with purely dwell time based and pupil-based selection modes, but this came at the price of more false positives when compared to the dwell time only approach. In a further demonstration (Strauch et al., 2017b), PATS enabled participants to complete eye-typing assignments with only minimal false positives, but performance metrics did not outperform those obtained with standard dwell time based interfaces. Together these examples show, perhaps for the first time, how eyes-only selective HCI systems can benefit from automatic detection of short-latency pupil dynamics.

\footnotetext{
${ }^{12} \mathrm{~A}$ video demonstration of the method can be accessed via the following link: https://www.youtube. com/watch?v=RHOyZFMI418.
} 


\subsubsection{Pupillometry in vigilance and visual search}

Whilst there are many pupillometry studies where the participants' task involved elements of vigilance, visual search or both, few have used pupil metrics specifically to explore performance and cognition in vigilance and visual search tasks of the kind outlined in Sections 2.2.1 and 2.3.1. One possible reason for this is that researchers have been deterred by methodological complications, particularly those which arise when attempting to use intricate visual paradigms to study cognitive influences on pupil size. Perhaps the most well known of these is the difficulty involved in dissociating cognitively induced effects on pupil size from those induced by changes in stimulus luminance or retinal and ambient illuminance; but there is also the issue of pupil foreshortening in some video-based eye tracking systems, whereby eye rotation leads to erroneous estimates of pupil size. Though not insurmountable, the measures for avoiding or overcoming such problems often impose design constraints which can be especially limiting for vigilance and visual search paradigms. Dealing with these issues is a central part of the current thesis, and more information on them is provided in the next chapter and the coming experimental chapters. The present section simply reviews the findings from existing pupillometric studies of vigilance and visual search, and asks what pupillometry may yet contribute towards our understanding of cognition in these tasks.

\section{Vigilance}

As noted previously, vigilance is considered to be a particular variety of attention characterised by sustained alertness, and is assessed in tasks which test a participants ability to respond quickly and accurately to stimuli over prolonged stretches of time. The two general classes of vigilance task outlined in Section 2.2.1 were the standard vigilance task, where participants respond to infrequent signals across lengthy uninterrupted periods, and the psychomotor vigilance task, where participants respond to more frequent signals over shorter periods. Although the pupillometry literature is sparse as regards these two specific tasks, with only a handful of studies to the authors knowledge (Beatty, 1982a; Kristjansson et al., 2009; Unsworth \& Robison, 2016), the evidence suggesting that pupil size may serve as a useful indicator of vigilant attention is far ranging. As noted previously, the vigilance decrement has been linked to arousal, sleepiness, fatigue and changing activity in the LC-NA system, all of which are reflected in the dynamics of the pupil. Further to this, some of the studies cited earlier in Section 2.4.2, which linked combinations of ocular metrics to declining vigilance performance, included pupil measures. Van Orden et al. (2000) showed that mean pupil 
size decreased as time-spent-on-task increased and McClelland et al. (2010) reported similar results. Another general finding from pupillometric studies of tasks requiring continuous or intermittent vigilance is that stimulus-evoked pupil responses reduce in amplitude as time-on-task increases (Hopstaken, van der Linden, Bakker, \& Kompier, 2015; Unsworth \& Robison, 2016; van den Brink et al., 2016). Taken together, this suggests that pupil measures, either in isolation or combination with other measures, may serve a useful purpose in real-time monitoring systems for predicting changes in performance caused by drowsiness, time-on-task, or declines in vigilant attention. Chapter 6 revisits this idea with a closer examination of the methods and findings of the above-cited studies and with two original experiments designed to examine the relationship between pupil and performance measures in vigilance tasks.

\section{Visual search}

Relatively few studies have measured pupil size in visual search tasks. Among the first to so this were Porter et al. (2007), who found that pupil size increased gradually as participants conducted searches, and that the magnitude of increase was affected by the difficulty of the search (as determined by set size and and distractor heterogeneity manipulations). On the basis of their findings they concluded that pupil size reflected the increasing effort required as search progresses, and they also suggested that their observed pupil responses may reflect the accumulation of memory load for previously visited item locations. Privitera et al.'s (2010) finding of transient pupil dilation following target detection with and without button-presses, discussed in Section 2.5.2, is also relevant in the context of visual search. This was an RSVP experiment, where target and distractor stimuli were presented in a central location and participants did not have to move their eyes, but similar findings have also been found in visual search under typical free-viewing conditions (Klingner, 2010a). Overall, the evidence suggests that pupillometry may serve as a useful means to explore cognitive mechanisms involved in search, but due to the complex nature of search tasks, the broad range of factors that cause the pupil to dilate (see Section 2.5.2) and various methodological complications (discussed in Chapter 3), it is a challenge to understand the relationship between observed pupil responses and the various task components. Chapters 4 and 5 aim to drive progress in this regard by using different forms of visual search task in combination with a range of methods and analysis techniques in order to identify an effective experimental approach for using pupillometry to investigate visual search processes. 


\section{Chapter 3}

\section{General methodology}

\subsection{Introduction}

This chapter provides detail on general aspects of eye tracking and pupillometry methodology, as well as data processing and analysis techniques, that are common to all of the experiments in this thesis. Specific methodological details for each experiment, such as those to do with stimulus preparation and experimental design, will be given in each of the respective methods sections. The data used to illustrate the pupillometry methods are derived from a simple experiment exploring the effects of three levels of luminance stimuli on the PLR, the details of which can be found in Appendix A.

\subsection{Eye-tracking methods}

\subsubsection{Infrared video-based eye tracking}

The problem of determining gaze position has been solved in many innovative ways throughout the history of eye tracking research. Today, the most popular solutions use computer algorithms to analyse the relative position of ocular features in video recordings made under infrared illumination. The dominant principle involves calculating the location of the centre of the pupil relative to the location of the glint on the cornea caused by the infrared light source. With the appropriate calibration procedures, these two reference points can be used to map gaze position on a two-dimensional plane with good accuracy. The centre of the pupil alone can be used to estimate gaze position, but accuracy in this approach will be compromised by head movements. The positional relationship between the centre of the pupil and corneal reflection changes 
with eye rotation and only minimally with head movements, allowing for eye and head movements to be disambiguated (Duchowski, 2007).

\subsubsection{The EyeLink 1000 system}

All eye tracking and pupillometry data in the current thesis were acquired using an EyeLink 1000 system (SR Research Ltd., Ontario, CA) in tower mount configuration (Figure 3.1a), which uses a chin and forehead rest for head stability. The EyeLink 1000 is an infrared video-based eye tracking system with an industry-leading specification, making it suitable for the current research. It is capable of 250,500 or $1000 \mathrm{~Hz}$ monocular video recording, determines gaze position with an average accuracy of $0.25^{\circ}$ visual angle and resolves pupil size to within $0.2 \%$ of the diameter (SR Research Ltd., 2010). It uses the pupil-corneal-reflection eye tracking principle outlined previously and implements two pupil detection models: Centroid and Ellipse Fitting. The former uses an algorithm to calculate and track the centre of the thresholded pupil mass, whereas the latter fits an ellipse to the pupil and tracks the centre of the ellipse. The Centroid model is recommended when using the system in tower mount configuration, and the Ellipse Fitting model is used exclusively for the remote configuration, but can also be useful in tower mount configuration for participants whose eyelids tend to partially obscure the pupil. Whichever algorithm is used, the output of pupil size data, which is given in arbitrary pixel units (area or diameter, depending on the choice of the operator), is always based on the Centroid model.
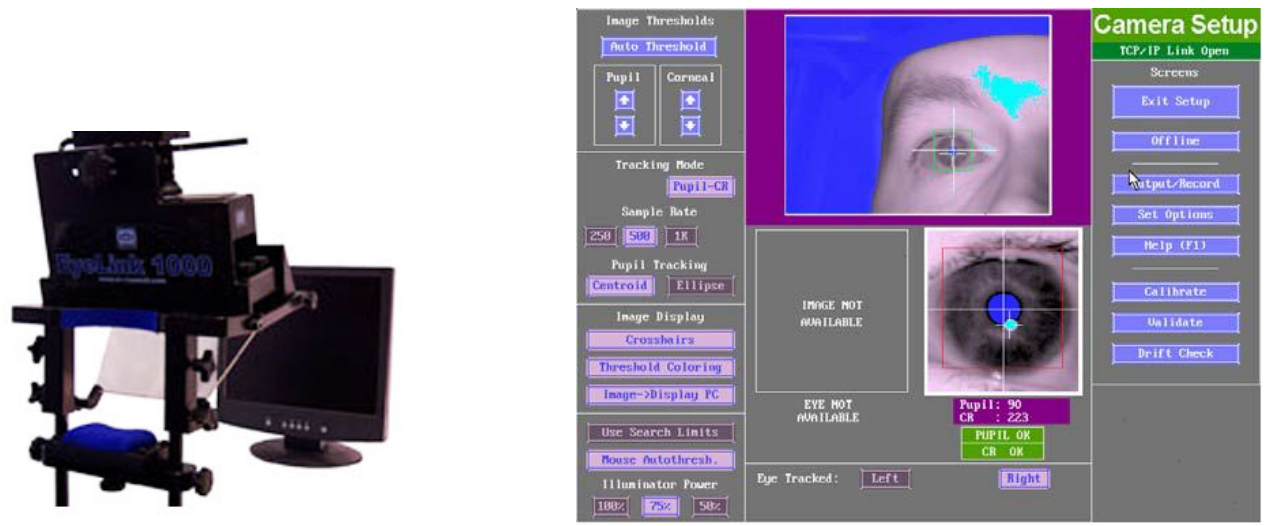

Figure 3.1. Typical view of the EyeLink 1000 system in tower mount configuration (left) and of the EyeLink Host user interface (right). 


\subsubsection{Saccadic thresholds and event parsing}

All eye tracking data in the current thesis were parsed on-line into meaningful ocular events (i.e. blinks, saccades and fixations) by the EyeLink parser, a proprietary event detection algorithm which uses velocity, acceleration and motion thresholds to detect saccades. The threshold parameters can be adjusted manually, but the EyeLink Host software offers two standard configurations of saccade sensitivity: 'NORMAL', which is suitable for cognitive research, and 'HIGH', for psychophysical research. The experiments in this thesis address questions to do with cognition and therefore make exclusive use of the 'NORMAL' configuration ${ }^{1}$, which implements a velocity threshold of $30^{\circ} / \mathrm{s}$, an acceleration threshold of $8000^{\circ} / \mathrm{s}^{2}$ and a motion threshold of $0.1^{\circ}$.

During recording, the parser checks the velocity and acceleration of the eye for each sample as it comes available. If either velocity or acceleration exceeds its given threshold, and the subsequent movement of the eye exceeds the motion threshold, the start of a saccade event is logged. The end of the saccade comes when both velocity and acceleration drop back below their thresholds. Samples in between saccades are grouped into fixations, and blinks are registered as periods of signal loss which occur between artefactual saccades caused by the rapid occlusion of the pupil by the eyelid and the rapid downward shift in the computed position of the pupil centre this causes. It is possible to parse data with other algorithms after it has been collected - an approach that is common in psychophysical studies of fixational eye movements (e.g. see Engbert \& Kliegl, 2003; Engbert \& Mergenthaler, 2006; Martinez-Conde, Macknik, \& Hubel, 2000)—but the experiments in the current thesis rely exclusively on the EyeLink parser for initial event identification.

\subsubsection{Hardware configuration and experiment programming}

Tower-mount configuration of the EyeLink system requires a computer to host the eye tracker and perform the eye tracking computations, and a display computer to run the experimental application, log behavioural responses, and communicate with the eye tracker via an Ethernet link. Schematic and photographic pictures of this setup from the laboratory are presented in Figure 3.2.

Experiment programming can be achieved in numerous ways due to multiple language support and compatibility with third-party software, but in this thesis, all experiments were programmed using Experiment Builder (SR Research Ltd., Ontario,

\footnotetext{
${ }^{1}$ The HIGH configuration implements a velocity threshold of $22 \%$ s, an acceleration threshold of $3800^{\circ} / \mathrm{s}^{2}$ and a motion threshold of $0.0^{\circ}$. This results in the detection of more saccades, but also increases the risk of false positives.
} 
CA), the proprietary software of SR Research. Experiment Builder has a graphical user interface with convenient 'drag and drop' functions for performing the most common actions required in eye tracking experiments, allowing the researcher to develop experiments with relative ease. Specialised functionality, when required, was added with custom scripts written in the Python programming language.
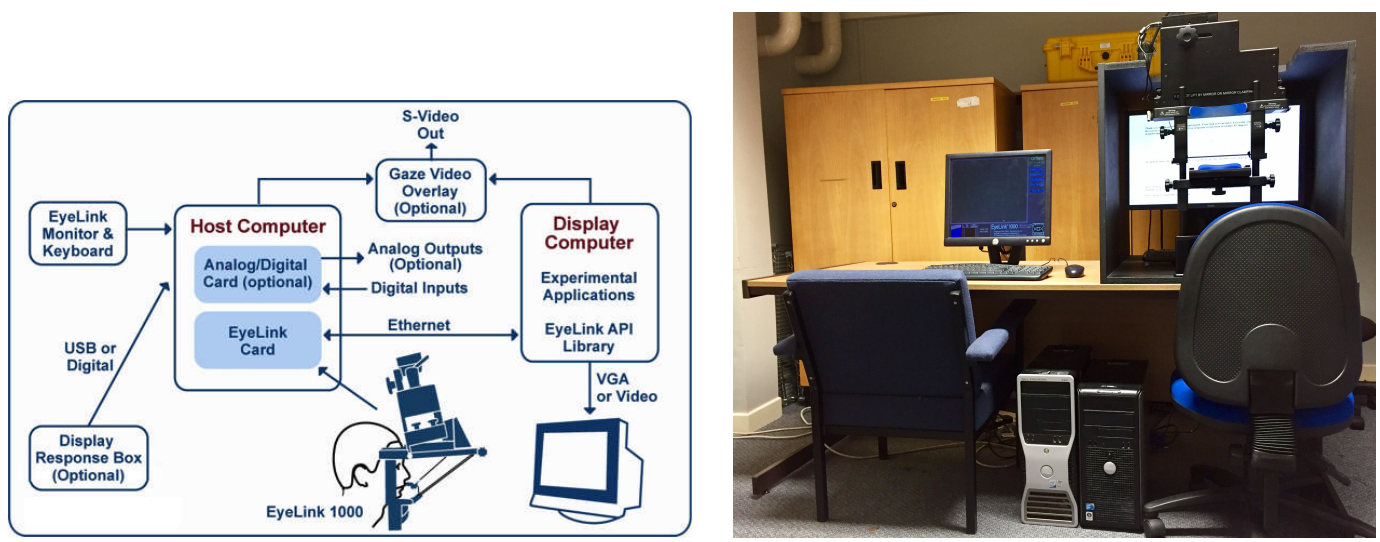

Figure 3.2. A schematic diagram illustrating the typical configuration of the EyeLink system (left) and a photograph of the laboratory setup used for most of the experiments in the thesis (right). The eye tracker and display are enclosed within a luminance tunnel, which shields the eyes from luminance changes and visual distractions in the periphery.

\subsubsection{Calibration, validation, drift checking}

Eye trackers must be calibrated in order to determine the relationship between the positions of the pupil and corneal reflection on the camera image and the gaze position on the display. This typically involves asking participants to fixate a number of targets which appear at various positions on the experimental display. For each fixation, the pupil-centroid position on the camera image is recorded, and the resulting set of target and pupil-centre positions is used as a model to compute gaze position. A validation routine can then be performed to check for problems with the calibration data before proceeding with data collection. This is similar to the calibration-a set of targets are presented for subjects to fixate, and the calibration model is used to estimate gaze position. Then, the difference between the location of the target and computed gaze position is calculated, which is the error. Gaze position errors arise mostly from physiological control of eye movements at the calibration stage, but noise and resolution in the eye tracking system also contribute. All calibration routines in the current thesis aimed for an average error of less than $0.5^{\circ}$ of visual angle, and less than $1.5^{\circ}$ for individual calibration points. 
During an experiment it is possible that the calibration model will become invalid, usually as a result of head movements, which means that the accuracy of gaze position recordings will be compromised. To test for this, drift checks are performed periodically. The purpose of a drift check is to assess the current validity of the calibration model during an experiment. A drift check involves presenting a single target for the participant to fixate and then calculating the distance between the target and the computed gaze position in degrees of visual angle. If the error is consistently large, then another calibration should be performed. The specific details of calibration, validation and drift checking routines are described individually for each experiment.

\subsubsection{Data output and handling}

The data recorded by the EyeLink system are saved in the proprietary EDF (EyeLink data file) format, which contains the raw data for each sample (i.e. $x$ and $y$ gaze coordinates, pupil size, velocity, acceleration), information about the events identified by the EyeLink parser, message output from the experiment program, and other useful information. This file can be viewed using the (also proprietary) DataViewer software (SR Research Ltd.), which has many convenient functions for exploring, visualising, and obtaining detailed reports on, the data. It is also possible to convert the EDF file to an ASCII text file using the 'EDF2ASC' utility supplied by SR Research, which allows for a more flexible approach using customised analysis scripts. In the current thesis, data were processed and analysed by combining DataViewer's functionality with the flexibility afforded by custom scripting in the Python and $\mathrm{R}$ programming languages. Numerous open source libraries were used, but extensive use was made of the SciPy Stack for Python (e.g. numpy, scipy, matplotlib, pandas) and Cili (Acland \& Braver, 2014), an eye tracking data tools package with convenient functions for handling pupillometry data.

\subsection{Pupillometry methods}

\subsubsection{Technical issues}

Methods for cognitive pupillometry have advanced considerably since the early studies which relied on hand measurement of low frequency photographic images of pupils (e.g. Hess \& Polt, 1960; Kahneman \& Beatty, 1966). This is largely thanks to advances in display technology, developments in stimulus presentation software, and the advent of high-speed eye tracking devices which yield accurate and precise measurements of 
pupil size. Yet even with this progress, pupillometry remains a delicate art, with a range of technical issues that can complicate experimental design and interpretation of data. In experiments using visual stimuli, two of the most important issues to consider are stimulus confounds and, if using a video-based eye tracker, pupil foreshortening.

\section{Stimulus confounds}

When drawing inferences about cognitive processes from pupil responses evoked by visual stimuli one must be confident that the observed effects are caused by experimental manipulations and not by any other properties of the stimuli. Luminance is the most obvious stimulus property that can affect pupil size, but research has also shown that the pupil can be affected by a range of other factors, including stimulus colour, spatial frequency, contrast, size and movement (Barbur, Harlow, \& Sahraie, 1992; Barbur, Wolf, \& Lennie, 1998; Goldwater, 1972; Kohn \& Clynes, 1969; Ukai, 1985; Watson $\&$ Yellott, 2012; Woodhouse, 1975). It is therefore important that stimuli are balanced between different conditions with respect to these visual properties. The experiments in the current thesis achieve this by making exclusive use of grey-scale stimuli and ensuring that luminance, spatial frequency and contrast are matched between conditions.

\section{Pupil foreshortening}

Cameras on video-based eye tracking devices view the eyes from a fixed position. When someone gazes straight down the optical axis of the camera, the full area of their pupil is projected onto the photographic sensor, ensuring that size estimates are closest to their true value. But when eye position deviates from the optical axis, the shape of the pupil as it appears from the optical perspective is distorted. If not accounted for in the system's software, this 'foreshortening error' guarantees that pupil measurements will be confounded to some extent by eye position (Brisson et al., 2013; Gagl, Hawelka, \& Hutzler, 2011; Hayes \& Petrov, 2015).

The EyeLink 1000 system does not compensate for pupil foreshortening. The user manual states that 'Pupil size measurements are affected by up to $10 \%$ by pupil position' (SR Research Ltd., 2010, pp. 98), and advises researchers interested in pupil measurements to design experiments where participants should not move their eyes during trials. While this approach may minimise the error, it is clearly a significant design constraint which does little justice to the ecology of human visual behaviour. For pupillometry experiments where stimuli appear across a large part of the screen, it is necessary to counterbalance or randomise the location of stimuli, and ideally, use a corrective procedure to reduce the impact of the error. Both of these approaches are 
adopted in the current thesis. The visual search and vigilance experiments in Chapters 4 and 6 involve centrally presented stimuli and do not permit eye movements during trials, whereas the visual search experiment in Chapter 5 involves stimuli which span a larger portion of the screen, permits eye movements, and corrects for pupil foreshortening with a data-driven method proposed by Brisson et al. (2013). A more detailed treatment of the foreshortening issue is given in Chapter 5 .

\subsubsection{Data processing}

As with many other forms of physiological measurement, pupil data must be processed before they are subject to statistical analysis. The three main processing stages used in the current thesis are blink interpolation, filtering and down-sampling. Event related analysis also requires the additional steps of epoching, baseline normalisation and averaging.

\section{Interpolation}

A video-based eye tracker can estimate pupil size only when the pupil is visible to its camera, so when a participant blinks during an experiment, signal is temporarily lost. This blink-related signal loss is clearly visible in the top panel of Figure 3.3 at the times where pupil size falls sharply to zero. Blinks can be treated simply as missing data, but an alternative that is used in virtually all published pupillometry papers is to estimate the values of missing data from the intact data surrounding each blink event. As noted by Mathôt (2013), though one can debate the theoretical meaningfulness of this approach, it has appreciable practical advantages when it comes to data analysis and visualisation.

The EyeLink parser used for blink detection in the current thesis records blink events as periods of complete signal loss nested between two artefactual saccades, which are the result of artificial motion caused by the progressive occlusion of the pupil by the eyelid. Data between the artefactual saccade events is unreliable (SR Research Ltd., 2010), so the minimum requirement is to interpolate between the start of the first saccade and the end of the second saccade (e.g. Hong, Walz, \& Sajda, 2014). As is evident in Figure 3.4, however, this approach often fails to capture aberrant data at the 'tail end' of the blink. To account for this, the current thesis employs a method proposed by Acland and Braver (2014), which extends the recovery index from the end of the second artefactual saccade to the first sample where the $z$-scored rate of change is less than $10 \%$ of the average rate of change within a $1000 \mathrm{~ms}$ moving window. 

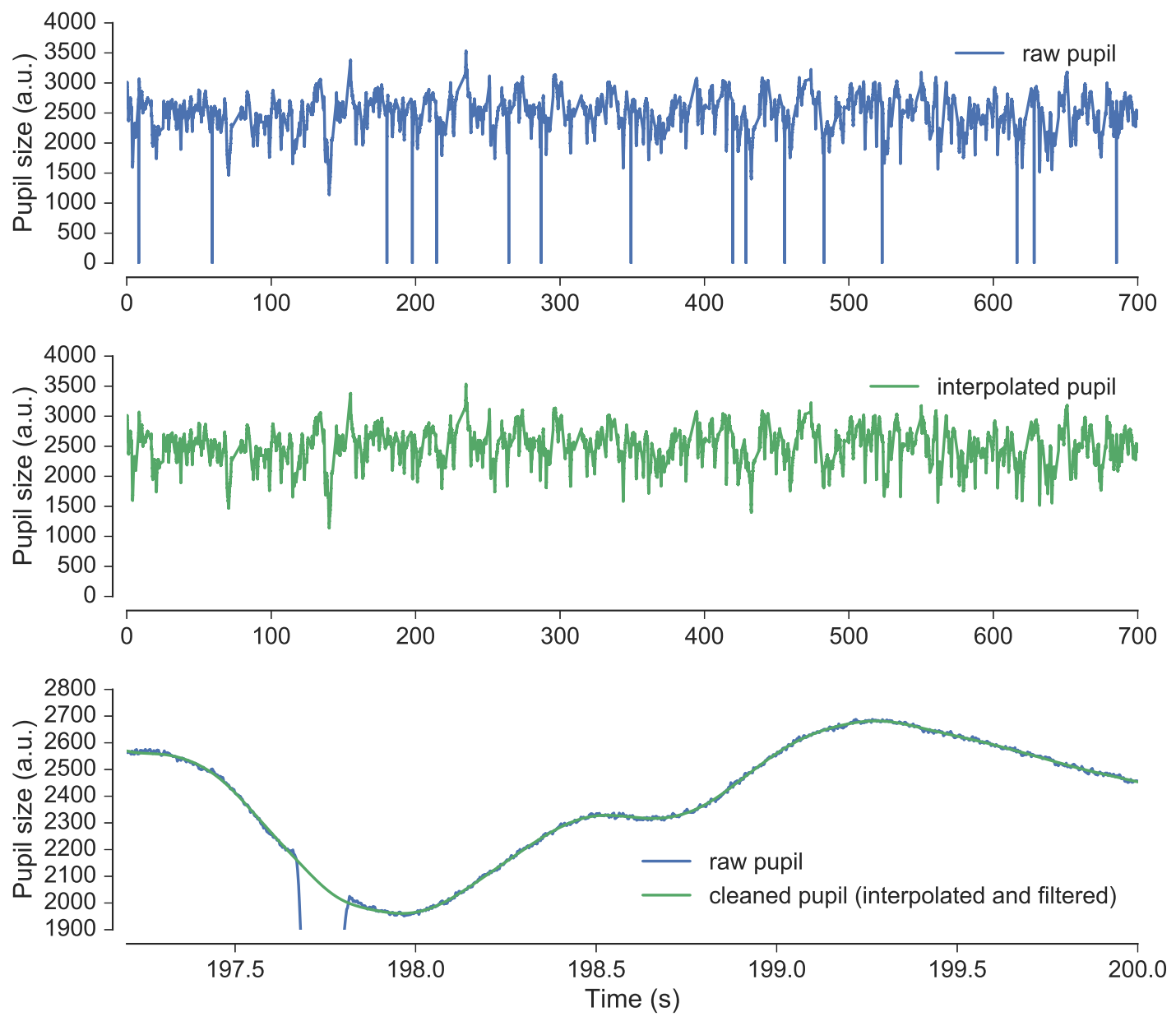

Figure 3.3. Cleaning the pupil data with linear interpolation and digital filtering. The top panel shows a section of the raw pupil time-course of a single participant in the experiment from Appendix A. Data loss caused by blinks can be seen clearly at the times where pupil size falls to zero. The middle panel shows the same time-course after applying a linear interpolation to the pupil data between the start and end times of each blink event identified by EyeLink's criteria, with some adjustment of recovery time. The bottom panel shows the effects of linear interpolation and $4 \mathrm{~Hz}$ filtering on a close-up of the same data. The cleaned pupil has no blink-related signal loss and is noticeably smoother than the raw pupil trace. 

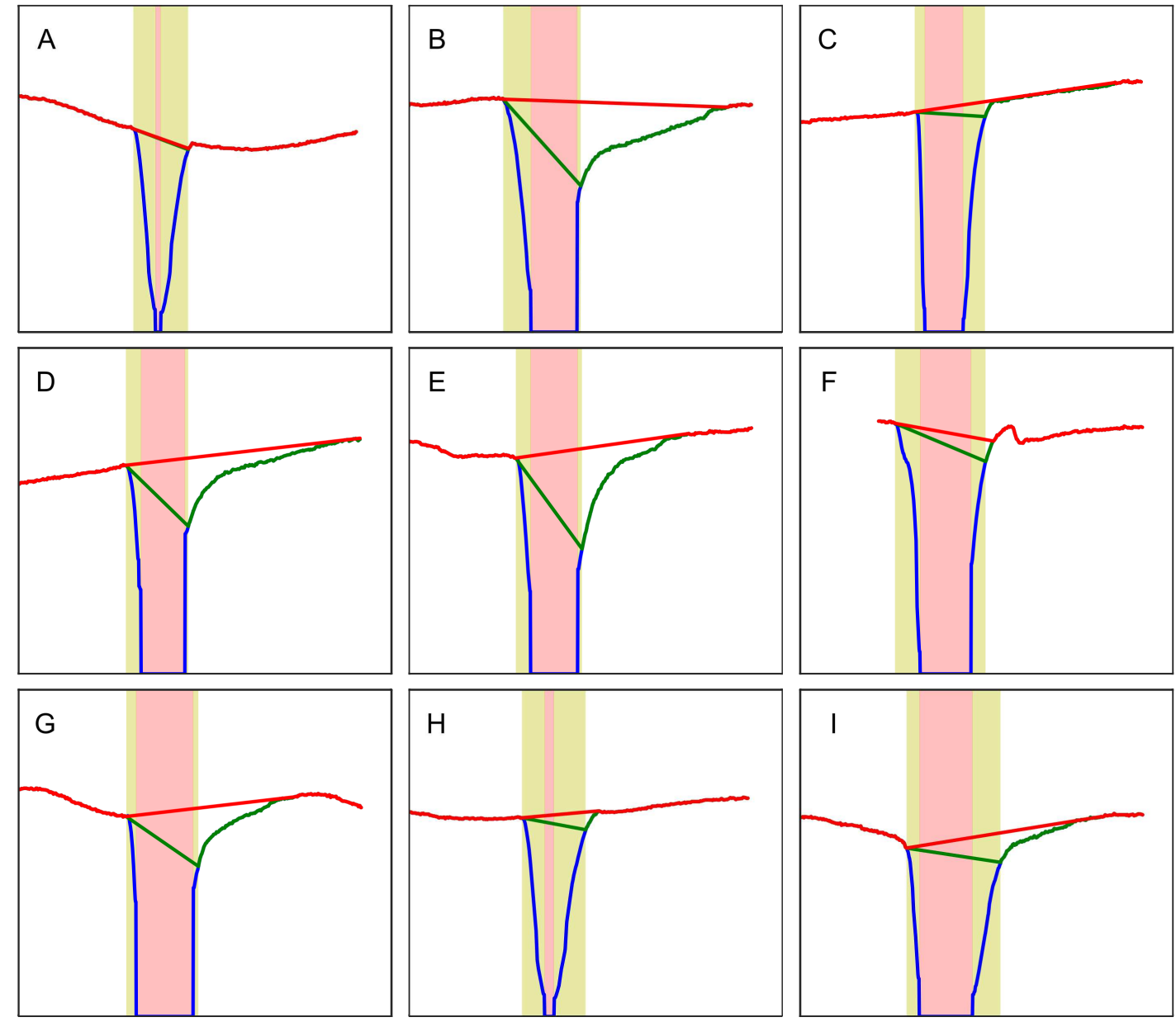

\section{- raw pupil basic interpolation}

z-threshold adjusted recovery

Figure 3.4. Schematic representation of 9 of the blink events from the top panel of Figure 3.3, showing two different approaches to linear interpolation (pupil traces are overlapping where only one colour is visible). Basic interpolation often fails to account for data which are still noticeably part of the blink artefact (e.g. for all but panel A). Acland and Braver's (2014) method of interpolation accounts for this by extending the recovery index of the blink from the last sample of the second artefactual saccade to the first sample where the $z$-scored rate of change is less than $10 \%$ of the average rate of change within a $1000 \mathrm{~ms}$ moving window. Vertical shaded regions indicate the temporal extent of artefactual saccades (yellow) and periods of complete signal loss caused by blink events (red). 


\section{Filtering}

The cognitive pupillary response is slow-moving and limited to a frequency range below $4 \mathrm{~Hz}$ (Hoeks \& Levelt, 1993; Loewenfeld, 1993), yet the pupil signal often contains higher frequency fluctuations, mainly the result of system measurement noise. Because these higher frequencies are thought not to reflect any cognitively meaningful information (see Kloosterman et al., 2015), filtering them from the data has become a standard practice. There are many ways to do this, but the present thesis adopts the common standard (e.g. de Gee et al., 2014; Kloosterman et al., 2015; Privitera et al., 2014) of applying a third order Butterworth filter with a $4 \mathrm{~Hz}$ cut-off to the pupil data. The effects of this processing step can be seen in the bottom panel of Figure 3.3, where the filtered pupil trace is noticeably smoother than the unfiltered trace.

\section{Down-sampling}

All pupillometry data in the current thesis were down-sampled to $50 \mathrm{~Hz}$ for ease of analysis. This sampling frequency is used commonly in the pupillometry literature (e.g. Hopstaken, van der Linden, et al., 2015; Klingner, 2010a; Nuthmann \& van der Meer, 2005; Porter et al., 2007; Starc, Anticevic, \& Repovš, 2017; van der Meer, Friedrich, Nuthmann, Stelzel, \& Kuchinke, 2003; Wierda et al., 2012) as it provides ample temporal resolution to capture the full range of pupil dynamics and a manageable amount of data for conducting statistical analysis.

\section{Epoching, baseline normalisation, averaging}

As with other continuous physiological variables like heart rate, EEG and skin conductance, the aim of most pupillometry experiments is to explore changes in pupil size with respect to stimulus exposure in different experimental conditions. This objective requires an event-related approach where fixed length epochs of pupil data are timelocked to the onset of specific periods of interest (e.g. at the appearance of a stimulus) and expressed in a form showing change from a baseline period around the onset of the period of interest. In the current thesis, this was achieved using the following formula:

$$
\% \text { change }=\frac{X \text { data }_{i}-\text { baseline }}{\text { baseline }} * 100
$$

where $X$ dat $_{i}$ is each sample in the epoch and baseline is the average pupil size during the baseline period. It is possible to convert arbitrary pixel units into absolute physical 
units, but this requires custom calibration with an artificial pupil of known size at the start of every recording session ${ }^{2}$.

Once the pupillometry data have been epoched and aligned to events of interest, the next step is to average together the data for each given time point within an experimental condition. This procedure attenuates variation in the pupil signal that is not related to the experimental manipulation. Averaging together the subject averages for a specific condition produces a grand-average waveform, such as those which are displayed in Figure 3.5.

\subsubsection{Statistical analysis}

Statistical methods are used to determine how likely it is that observed results are due to chance. A common approach with pupillometry data is to calculate a summary statistic for a chosen interval, such as the mean or maximum pupil size in the two seconds after stimulus presentation, and then use frequentist statistical methods such as $t$-tests and analysis of variance (ANOVA) to test for significance (e.g. Aboyoun \& Dabbs, 1998; Ahern \& Beatty, 1979; Bradley et al., 2008; Heaver \& Hutton, 2011; Hess \& Polt, 1960; Johnson, 1971; Kahneman \& Beatty, 1966; Otero et al., 2011; Partala \& Surakka, 2003; Richer \& Beatty, 1985; Simpson \& Molloy, 1971; Unsworth $\&$ Robison, 2017). Another approach that is becoming more common in recent years (e.g. de Gee et al., 2017; de Gee et al., 2014; Kloosterman et al., 2015; Knapen et al., 2016; Privitera et al., 2014; Privitera et al., 2010) is to assess the statistical significance of experimental effects over time - that is, in a number of time-bins in the peri-stimulus interval. This has a number of advantages. First, it is not always obvious prior to data collection which interval should be used to calculate summary statistics, and the post hoc decision over which interval to use can be a source of experimenter bias. Second, characteristics of nonluminance-mediated changes in pupil size (e.g. temporal locus, magnitude, duration) depend on their neurocognitive origins. Sometimes they are small and transient (e.g. Privitera et al., 2010; Wang \& Munoz, 2014), whereas other times they are large and protracted (Kahneman \& Beatty, 1966; Porter et al., 2007). Using a fixed interval may fail to capture the full range of experimental effects. Finally, pupil size is a continuous variable, and as such it is much more natural and informative to explore the significance of experimental effects with respect to time.

\footnotetext{
${ }^{2}$ To convert the arbitrary units to absolute values, one could print out a black dot of known size (e.g. $8 \mathrm{~mm}$ ) with a laser printer to serve as an artificial pupil. This artificial pupil could then be placed as close as possible to the participant's eye at the start of the session and a short recording made. It would then be possible to perform a linear interpolation of the participant's actual pupil size based on this value.
} 
The main disadvantage of conducting statistical tests on pupil time-courses as opposed to summary statistics is that a larger quantity of tests must be carried out, which dramatically increases the probability of a type 1 error (i.e. a false positive) if conventional $t$-test statistics are used. For instance, the experiment outlined in Appendix A explored the effects of three incrementing levels of luminance on pupil modulation. All luminance stimuli reliably evoked a PLR which unfolded over the course of about three seconds, results which are clearly visible in Figure 3.5. Assessing the significance of change from baseline for each condition could be achieved by conducting a two-tailed $t$-test against the null hypothesis that the pupil mean for each specific time-point is not different from the baseline prior to the onset of the luminance increment. With data downsampled to $50 \mathrm{~Hz}$, this requires $150 t$-tests, substantially raising the probability of a type- 1 error. The standard approach to correct for this would be to apply the Bonferroni correction, whereby the critical value for significance is divided by the number of tests carried out. With the present example, this lowers the threshold for significance from .05 (the conventional threshold used throughout the thesis) to .0003 , meaning that only time points where $p<.0003$ would pass the threshold for significance. This is a heavy penalty, and amounts to a substantial reduction in statistical power (e.g. Nakagawa, 2004).

The experimental work in the current thesis avoids both the multiple comparisons problem and the Bonferroni correction by using cluster-based nonparametric permutation tests - as described by Maris and Oostenveld (2007) — to assess the probability of significance for pupillometric effects expressed as a function of time. Such tests are used commonly with neuroimaging data (Blair \& Karniski, 1993; Bullmore et al., 1999; Guthrie \& Buchwald, 1991; Holmes, Blair, Watson, \& Ford, 1996; Kaiser \& Lutzenberger, 2005) and have been used increasingly for pupillometry data in recent years (e.g. Kloosterman et al., 2015; Knapen et al., 2016; Privitera et al., 2010; Starc et al., 2017). They are flexible in nature, and their validity does not depend on the probability distribution of the data or the type of statistical test that is used to quantify an experimental effect (Maris \& Oostenveld, 2007). The general procedure for conducting these tests is described next, with an example implementation on the data from the simple pupillometry experiment described in Appendix A. For a more thorough description of this statistical method, complete with mathematical proof of how it controls for the multiple comparisons problem, please refer directly to Maris and Oostenveld (2007). 


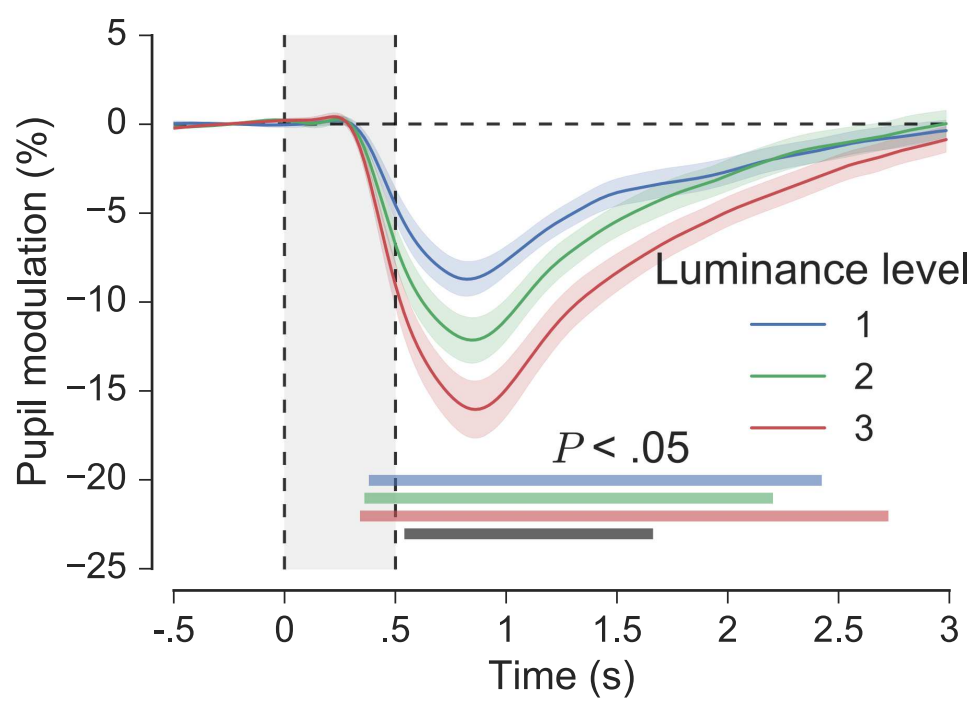

Figure 3.5. Grand average pupil responses to stimuli with three incrementing levels of luminance (see Appendix A for details). The light-grey shaded area spanning the $y$-axis denotes the onset and duration of the luminance increment, and the shaded areas surrounding the pupil traces show the standard error of the mean (SEM, bootstrapped, 5000 iterations). The horizontal coloured bars show, for each luminance level, clusters of significant change from baseline as identified by nonparametric permutation tests (1024 permutations, $p<.05$, cluster-corrected). The dark horizontal line denotes the period at which a significant main effect was present ( $F$-test, 1024 permutations, $p<$ $.05)$. 


\subsubsection{General procedure for cluster-based nonparametric permu- tation tests}

The general procedure for conducting nonparametric permutation tests to assess the probability of significance for a difference between two experimental conditions is as follows (adapted from Maris and Oostenveld, 2007): (1) Gather the observations for the two experimental conditions together in a single set; (2) randomly partition this set into two subsets, each containing as many observations as there were in the original two conditions; (3) compute the test statistic on the random partition; (4) repeat the previous two steps many times and build a histogram of test statistics; (5) determine the $p$-value as the proportion of partitions that resulted in a more-extreme test statistic than that which was originally observed between the two conditions; (6) if the $p$-value is smaller than the alpha threshold for significance, reject the null hypothesis. As with traditional frequentist statistical methods, the probability of this procedure leading to the false rejection of a well-specified null hypothesis is equal to the critical alpha threshold.

When using nonparametric permutation tests with pupillometry time-series, where an effect can be present at multiple time-samples, it is necessary to use a statistic which controls for the multiple comparisons problem. One solution to this is the clustermass statistic (e.g Bullmore et al., 1999), which involves the clustering of contiguous time-samples that show similar differences in magnitude and sign. The process for calculating the cluster-mass statistic is as follows (adapted from Maris and Oostenveld, 2007): (1) For each sample, compute a test statistic which quantifies the experimental effect between the conditions (e.g. a $t$-test or an $F$-test); (2) select all samples where test statistics surpassed a predetermined threshold (e.g. the critical value from the sampling distribution of the test statistic under the appropriate degrees of freedom); (3) cluster all of the selected samples together into contiguous sets; (4) calculate the cluster-mass by summing all of the test statistics within each cluster; (5) take the largest of the cluster-level statistics; (6) estimate the $p$-value using the general permutation procedure described in the previous paragraph.

The procedures described above are used throughout the thesis to assess the probability of significance for experimental effects in pupillometry time-series. The tests were implemented with functions from the MNE package for the python programming language (Gramfort et al., 2013; Gramfort et al., 2014). To illustrate the use of these tests, an example is provided in Figure 3.5, where the aim was simply to evaluate the statistical significance of pupil modulation from baseline for three incremental luminance stimuli, and also the main effect of Luminance Level (further details in Appendix A). Significant modulation from baseline for each luminance condition was 
assessed using one-sample $t$-tests with a comparison value of 0 (i.e. the average pupil modulation in the baseline period), and the main effect of luminance was assessed with $F$-tests. The results clearly show that all luminance stimuli caused significant change from baseline, and also that there was a significant main effect of Luminance Level, whereby constriction was greater as luminance increased.

\subsection{Summary and overview of experimental chapters}

Up to this point, the primary aims, key topics, and general methods of the thesis have been described. Chapter 1 introduced attention and WM, vigilance, visual search, eye tracking and pupillometry within the contexts of psychological research and practical relevance to real-world issues. Chapter 2 elaborated on the theory and experimental findings which underpin the current state of scientific understanding regarding these topics, and the present chapter has covered the general eye tracking and pupillometry methods that are used throughout the thesis. The next three chapters present the results of original experiments designed to explore how changes in pupil size relate to various aspects of performance in visual search and vigilance tasks. Chapters 4 and 5 explore how the different cognitive processes contribute to pupil dilation in visual search tasks, with emphasis on the methodology required for controlling stimulus confounds and pupil foreshortening. Chapter 6 explores how pupil measures relate to performance measures in vigilance tasks, focusing on the PVT and 'standard' vigilance task frameworks. Finally, Chapter 7 considers the methodological and theoretical implications of experimental outcomes. 



\section{Chapter 4}

\section{Pupillometric effects of effort and target detection in visual search with brief displays}

\subsection{General introduction}

This chapter and the next are concerned principally with what pupillometry can reveal about the cognitive processes involved in visual search. As noted in Section 2.5.5, a limited number of studies have explored this to date, possibly due to difficulties in dealing adequately with confounds associated with visual stimuli and the pupil foreshortening error (PFE) in video-based eye trackers (see Section 3.3.1). Further to this, even where adequate control can be exerted over these confounds, pinpointing any observed pupil effects to a specific cognitive mechanism is by no means trivial, because visual search involves many distinct component processes which may contribute individually to pupil dilation. For example, a typical visual search task may involve orienting attention around a display, detecting targets and distractors, deciding whether a target is present or absent, and indicating this decision with a button-press. Search may also involve memory processes to some extent, and harder searches may require more cognitive effort than easier searches. The evidence that was reviewed in Section 2.5.2 suggests that each of these search processes is likely to make its own individual contribution to pupil dilation, but of the few pupillometric studies of visual search, none have attempted to pull any of these effects apart.

A better understanding of how visual search processes are differentially reflected in pupil dilation patterns would have two main advantages. First, it would enhance the utility of pupillometry as a tool for use in laboratory-based studies of visual search by al- 
lowing researchers to target increasingly specific questions and make more theoretically informed interpretations of experimental findings. Second, it would provide a better idea as to the scope for using pupil metrics for applications in operational environments, like medical image analysis and X-ray baggage screening, where tasks are generally more complex than those used in laboratory settings. The present chapter focuses specifically on two component processes of visual search that have been recognised to contribute to pupil dilation: effort and target detection. The evidence for these effects on pupil dilation was covered in some detail at various points throughout Section 2.5, but here it is reviewed in greater depth.

\subsubsection{Pupillometric effects of effort in visual search}

The general link between pupil dilation and cognitive effort has long been established (e.g. Beatty \& Wagoner, 1978; Kahneman, 1973; Kahneman \& Beatty, 1966, 1967), but only relatively recently was it demonstrated that pupil dilation reflects the variable effort required for visual search tasks of different difficulty. Porter et al. (2007) conducted two experiments in which participants searched for forward facing 'C's among distractor 'C's facing other directions. In the first experiment, participants had to report whether a target was present or absent, and search difficulty was manipulated by varying the set size ( 9 or 10 vs. 29 or 30) and heterogeneity of distractors (1 vs. 3 fixed distractor orientations). In the second experiment, participants had to report whether 1 or 2 targets were present in large set sizes ( 29 or 30 ) with heterogeneously oriented distractors. Both experiments also included a 'counting' condition, where the task was simply to count the number of display elements and report whether the total number was odd or even. The two main findings across all search conditions in both experiments were that (a) pupil size increased gradually from stimulus onset until the response, corresponding closely with the period of search-related processing, and (b) the extent of pupil dilation was greater for the more difficult searches as compared to the easier searches. Interestingly, the most pronounced pupil responses were observed in the counting conditions, which lead the authors to suggest that the variable effort required across all of the conditions was due to the differential load placed by each of them on locational memory ${ }^{1}$. Further evidence for this interpretation came from the second experiment when comparing present-absent search with 1- vs. 2-target search, where pupil dilation in both conditions followed similar patterns but was boosted slightly towards the end of the search in the

\footnotetext{
${ }^{1}$ Accuracy in counting required perfect locational memory from the outset to avoid missing or double-counting an item whereas accuracy in the present-absent search for targets was not dependant on locational memory.
} 
1- vs. 2-target condition, suggesting an increased accumulation of locational memory load (i.e. memory for the location of the first target).

Porter et al. (2007) were able to make informed interpretations of their pupil data in terms of the effects of underlying cognitive processes because of the high level of control that they exerted over their stimuli and task. Crucially, stimuli were monochrome and the background was kept at a constant brightness, minimising the confounds associated with the pupil's visual reflexes (discussed in Section 3.3.1). A mask consisting only of the background brightness was also presented prior to each search array, which adapted the pupil to the general luminance levels and minimised the luminance transition between displays solely to that which was caused by the appearance of the elements in the array. These methodological precautions enabled the authors to interpret the observed pupil responses as a reflection of the moment-tomoment cognitive processing involved in the search task. The experiments of Porter et al. were the first to demonstrate that pupil dilation can be used as a valid measure of effort in visual search tasks without strict control over gaze position and without exact matching of the low-level visual properties of stimuli. Subsequent studies conducted in the same lab built on the success of these original experiments, using pupil size as an index of the cognitive effort involved in feature and conjunction search in healthy young and old participants (Porter, Tales, et al., 2010), as well as patients with Alzheimer's disease (Porter, Leonards, et al., 2010). Other than these examples however, the use of pupil size to study cognition in free-viewing visual search is rare.

\subsubsection{Pupillometric effects of target detection in visual search}

As mentioned previously in Section 2.5.2, Privitera et al. (2010) observed transient pupil dilation following target detection in two RSVP experiments. In the first experiment, participants monitored a stream of isoluminant object icons $\left(2^{\circ} \times 2^{\circ}\right.$ of visual angle $)$ presented at the centre of a computer screen at a frequency of $0.7 \mathrm{~Hz}$ and had to detect and respond via button-press to icons that could be semantically associated with a given word—either music, brain, or holiday — selected randomly for each participant prior to the start of the experiment ${ }^{2}$. In the second experiment, participants monitored streams of grey-scale images depicting rural and urban areas presented at a frequency of $10 \mathrm{~Hz}$, some of which contained a target helipad symbol at the centre of the image, usually atop a large structure in an urban setting. Here the task was to detect the iconic targets and

\footnotetext{
${ }^{2}$ For example, music could be associated with a picture of a trumpet or a musical note, brain with a picture of a dolphin or a dog, and holiday with a picture of a candle with Christmas holly or a Christmas stocking.
} 
respond via button-press upon detection. The participants in the first experiment had no special training, but those in the second experiment were professional image analysts trained in the analysis of satellite imagery. In both experiments, some of the participants completed a rerun where they simply had to count the targets or keep looking for targets (i.e. they were not required to report detection with a button-press response). The main finding across both experiments was that target detection evoked transient pupil dilation which began between 300-700 ms following target presentation, peaked on average between 1-2 s after presentation, and returned to baseline levels between 2-3 s. This effect was most pronounced in conditions requiring an overt detection response, but it was also significant, albeit attenuated, in the rerun conditions where no response was required. Additional findings to emerge from the second experiment were that the magnitude of dilation following target detection varied as a function of target density ${ }^{3}$ and the time at which the target appeared in the viewing session (larger dilations occurred in sessions with fewer targets and for targets viewed earlier in the session), also that the pupil dilated significantly even when a target was missed in the button-press reporting condition. This latter finding prompted the authors to raise the possibility that pupil dilation may reflect the presence of a target that was not reported as being seen, but may yet have registered at some level of consciousness.

Whilst many studies have reported pupil dilation around the time of a motor response indicating some aspect of task fulfilment (e.g. Einhäuser et al., 2010; Hupé et al., 2009; Kloosterman et al., 2015; Richer \& Beatty, 1985, note also Section 2.5.2), the work of Privitera et al. was among the first to clearly demonstrate an effect of target detection even when the requirement for such a response is absent (but see Hakerem $\&$ Sutton, 1966, for a possible earlier example using close-to-threshold luminance stimuli). Two further studies by Klingner (2010a) and Wierda et al. (2012) corroborate Privitera et al.'s finding. In Klingner's study, participants conducted a serial search for target 'L's among 'T's and reported how many targets were present (i.e. 0, 1, 2, or 3) via mouse-click at the end of the trial. Using a technique he called fixation-aligned pupillary response averaging, where segments of pupil data are selected on the basis of gaze data, temporally aligned and averaged together, Klingner found that fixations on targets evoked transient pupil dilations similar to those observed by Privitera et al. (2010), whereas fixations far from targets did not cause the pupil to dilate. In the study by Wierda et al., participants performed a standard attentional blink task ${ }^{4}$, searching for

\footnotetext{
${ }^{3}$ The experiment was organised into multiple two-minute viewing sessions, each containing different quantities of targets, ranging uniformly between one every $60 \mathrm{~s}$ to one every second.

${ }^{4}$ The attentional blink paradigm is an RSVP paradigm which was developed simultaneously by Broadbent and Broadbent (1987) and Weichselgartner and Sperling (1987), and later refined and popularised by Raymond, Shapiro, and Arnell (1992). In a typical experiment, participants undergo many trials where
} 
upper-case target letters embedded in streams of rapidly presented $(10 \mathrm{~Hz})$ nontarget digits and reporting the target identity (or identities, in trials with two targets) at the end of the trial. The relevant finding in this experiment was that trials containing targets evoked significant transient pupil responses, whereas trials without targets did not ${ }^{5}$.

\subsubsection{Summary and current direction}

The research outlined above provides convincing evidence that pupil dilation during visual search reflects cognitive processes relating to effort and target detection. Specifically, harder searches cause the pupil to dilate more than easier searches (Porter et al., 2007), and target detection is associated with transient pupil dilation, even in the absence of an overt detection response (Klingner, 2010a; Privitera et al., 2010; Wierda et al., 2012). What is unclear from this evidence however is the relative extent to which each of these factors contribute to pupil dilation during visual search and how the effects interact. Porter et al. (2007) manipulated search difficulty and target presence in their experiments and observed that target-present trials evoked higher peaks at the time of response, but they did not explore this effect in detail; Klingner (2010a) reported target detection effects in visual search but did not manipulate search difficulty; and Privitera et al. (2010) and Wierda et al. (2012) noted effects of target detection in RSVP experiments where individual targets and distractors were presented sequentially, but also without systematic control over task difficulty. Therefore, what is known about the effects of effort and target detection on pupil dynamics during visual search comes from

they are presented with a rapid stream of alphanumeric characters (e.g. 10 characters per second for 2 s) and must then respond at the end of the trial as to the identity / presence of two targets-labelled T1 and T2 - that were embedded in the stream. For example, participants might have to report the identity of two uppercase letters embedded in a stream of 18 digits. Crucially, the distance between T1 and T2 is varied across trials, and accuracy in detecting the targets is explored as a function of this distance. The key finding is that whilst participants are generally good at detecting T1, accuracy in detecting T2 depends on its proximity to T1. Specifically, Raymond et al. (1992) reported that accuracy in detecting $\mathrm{T} 2$ is especially poor when it is presented between 180 and $450 \mathrm{~ms}$ after T1, suggesting that the time taken to process T1 leads to a temporary suppression of visual processing. Raymond et al. (1992) coined the term 'attentional blink' to describe this effect, because it is akin to that which might be expected if T1 triggered an eye-blink.

5 Also of general interest from this report is Wierda et al.'s novel 'automated dilation deconvolution' approach to analysing pupillometry data, which enabled them to track the effects of attention on the pupil with a greater temporal resolution than can be achieved with standard pupil waveforms. For example, in trials with two targets (well separated enough to allow dual detection), the standard pupil waveforms showed only a single peak, presumably reflecting the superposition of the attentional effects related to each individual target. However, when the pupil response was modelled as a function of a series of cognitive events using automated dilation deconvolution, clear peaks relating to each target were observed. Although not used in the current thesis, Wierda et al.'s method of analysis shows that in principle it is possible to resolve attentional effects on the pupil which arise from multiple temporally proximate events. 
Pupillometric effects of effort and target detection in visual search

a limited number of experiments, each of which used different methods and focused exclusively on one of the two effects. Consequently it is unclear whether the small and transient effects of target detection would be masked by the larger and more sustained effects of cognitive effort arising from search difficulty. This particular question-which is the focus of the current chapter-has considerable bearing on the feasibility of using pupil dilation as an index of target detection in HCI systems, where practical utility would ultimately depend on the ability to obtain reliable effects of target detection across a range of task conditions with varying difficulty.

A method for simultaneously exploring the effects of effort and target detection on pupil size in visual search must deal adequately with the confounds associated with visual stimuli and pupil foreshortening, both of which were outlined in Section 3.3.1. The aforementioned studies differed with respect to how this was achieved. Each of them controlled for the effects of stimulus luminance by using either balanced monochrome or isoluminant stimuli, but there were key methodological differences to do with stimulus presentation and measurement devices which have bearing on the issue of pupil foreshortening. Porter et al. (2007) and Klingner (2010a) used a typical visual search protocol where participants had to make eye movements to explore the stimulus array, meaning that foreshortening of the pupil image will have occurred. The extent to which this was problematic to their analyses is unclear. It may have been mitigated by corrective measures built into the measurement hardware, such as a pixel-to-millimetre conversion accounting for distance between the eye and camera ${ }^{6}$, but this was not discussed in either of the reports. On the other hand, Privitera et al. (2010) and Wierda et al. (2012), who obtained pupil measurements with the same system used to obtain pupil measurements in the current thesis-an EyeLink 1000 (SR Research Ltd., Ontario, CA), which does not compensate for pupil foreshortening (note Section 3.3.1)—presented targets and distractors sequentially at a central location on the screen and instructed participants not to move their eyes during trials. This is a common approach for eliminating the confound, but also one which constrains experimental design in a way that is problematic for typical visual search paradigms.

\footnotetext{
${ }^{6}$ Klingner (2010a) obtained pupil measurements with a Tobii 1750 remote system which calculates pupil size as the length, in pixels, of the major axis of an ellipse fitted to the camera image of the pupil and converts this measure to millimetres using a scaling factor derived from inter-pupillary distance. This method may not be affected by perspective distortion (Köles, 2017), but it is still subject to errors arising from individual differences in pupil shape and iris anatomy (Gagl et al., 2011). Porter et al. (2007) obtained pupil measurements with a locally developed head-mounted infrared video system which output measurements of horizontal pupil diameter in millimetres. This suggests that a pixel-to-millimetre conversion was implemented by the recording system, but that it may not have accounted for measurement errors arising from changes to horizontal gaze position. The implications of this within the context of Porter et al.'s design and experimental findings will be discussed in the following chapter.
} 
Clearly then, the issue of pupil foreshortening presents a dilemma in the context of visual search which must be addressed at the early stages of design. Ideally, a researcher who wishes to obtain pupil measurements in a standard free-viewing search paradigm should use a measurement system which compensates adequately for gaze position in its estimation of pupil size. Where this is not possible, the effects of gaze position should be apprehended during stimulus and task design (e.g., by ensuring that the effect is not rendered systematic by the positioning of display elements or idiosyncratic search strategies) and a corrective routine should be implemented after the data has been collected (e.g. Brisson et al., 2013; Gagl et al., 2011; Hayes \& Petrov, 2015; Pomplun \& Sunkara, 2003). This approach is adopted in Chapter 5, but the experiments in the current chapter tackle the problem by using the alternative visual search paradigm that was outlined in Section 2.3.1, where compact search arrays are presented for brief durations close to the point of fixation and participants are instructed not to move their eyes during trials (e.g. Bergen \& Julesz, 1983a, 1983b; Braun \& Sagi, 1990; Eriksen \& Spencer, 1969; Palmer, 1994; Palmer et al., 1993; Sagi \& Julesz, 1985; Shiffrin \& Gardner, 1972; Sperling, 1960). This form of visual search is different in that the search is conducted on the internal representation of the stimulus, without eye movements, in order to determine whether a target was present or absent (Wolfe, 1998a). Although this is generally a constrained approach, it eliminates the confound of pupil foreshortening and affords the flexibility to explore the pupillometric effects of effort by manipulating search difficulty (e.g. by varying either set size, distractor heterogeneity or both), and target detection by varying whether a target was present or absent in the array (e.g. a target prevalence of 50\%). Based on the research discussed above, one would generally expect to observe the smallest pupil responses for less-difficult searches without targets and the larger responses for more-difficult searches with targets.

\subsection{Experiment 1}

\subsubsection{Introduction}

The aim of this experiment was to assess the quantitative and qualitative effects of effort and target detection on the dilatory patterns of the pupil in a visual search task with brief stimulus displays. To this end, a simple and straight-forward approach was adopted whereby effort and target detection were explored through manipulating two factors: Set Size (4 vs. 6) and Target (present vs. absent). Small set sizes ensured that all display elements could be presented close to the point of fixation and that VWM would not be stretched too far beyond its known capacity of 4-5 objects (Luck \& Vogel, 1997; 
Phillips, 1974; Sperling, 1960; Vogel et al., 2001). Participants were asked to search for forward facing Landolt ' $C$ 's in arrays of backward facing 'C's and to indicate for each trial whether a target was present or absent, as in Porter et al. (2007). It was predicted that (i) participants would find searches to be more difficult for the larger set sizes due to the capacity limitations of VWM, and that this would be evident from reduced accuracy and perceptual sensitivity (i.e. $d^{\prime}$ ), longer RTs and larger pupil responses; and (ii) pupil responses would be larger for target-present compared to target-absent searches. Also of interest was whether the pupillometric effects of Set Size and Target would interact, and whether they would have distinct signatures in the waveforms observed immediately after stimulus exposure.

\subsubsection{Method}

\section{Participants}

Thirty participants completed the experiment, but 7 were excluded from the analysis due to data quality and task performance issues (see below in Section 4.2.2). Of the 23 that remained, age ranged between $18-36$ years $(M=23.5, S D=3.7)$ and 21 were female. All participants were students at Swansea University reporting normal or corrected-tonormal acuity and colour vision. Participation was voluntary or in exchange for course credit. The experimental protocol was approved by the Ministry of Defence Research Ethics Committee and the Department of Psychology Ethics Committee at Swansea University. Written informed consent was obtained from each participant.

\section{Design}

Two factors were manipulated in a repeated-measures design: Set Size (4 vs. 6) and Target (present vs. absent). Participants searched for forward facing 'C's in compact stimulus arrays of backward facing 'C's presented for $250 \mathrm{~ms}$ close to the point of fixation. It was deemed that $250 \mathrm{~ms}$ would be short enough to prevent large eye movements (e.g. Bergen \& Julesz, 1983b) and long enough to evoke a VWM representation of a sufficient quality for search (e.g. Luck \& Vogel, 1997; Vogel et al., 2001). Following stimulus exposure, participants had to indicate whether a target was present or absent in the array. A single set of 128 stimulus arrangements were used in the experiment. These consisted of 64 target-present and 64 target-absent arrangements for both of the set sizes (i.e. a target prevalence of 50\%). Participants completed 256 trials (each image presented twice) in a single testing session lasting approximately $40 \mathrm{~min}$. The trials were organised into 8 blocks of 32, with each block containing 8 
trials of the four conditions exemplified in Figure 4.1. The order of conditions was randomised within each block. Accuracy and RT (from stimulus onset until response) were recorded for each trial, as well as continuous recordings of gaze position and pupil size. The task was performed in a quiet room with dim lighting.

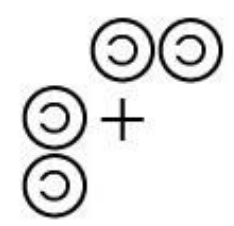

Set Size: 4 Target: absent

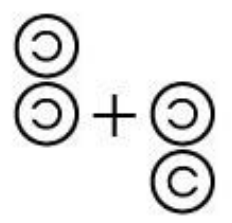

Set Size: 4

Target: present

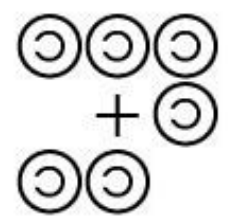

Set Size: 6

Target: absent

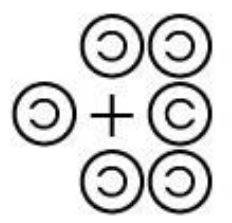

Set Size: 6

Target: present

Figure 4.1. Example search arrays for the four conditions in Experiment 1. The target was a forward facing ' $C$ ' and distractors were backward facing ' $C$ 's. All display items were allocated randomly to 8 possible locations on a grid surrounding the fixation cross, with the target appearing at each location an equal number of times throughout the experiment. All conditions occurred with equal prevalence.

\section{Stimuli and apparatus}

The stimuli were arrays of Landolt 'C's enclosed within an unbroken ring (Hooge \& Erkelens, 1996; Porter et al., 2007), clustered tightly around a central fixation cross (e.g. see Figure 4.1). They were created using vector graphics software and custom python scripts. Individual target and distractor image tiles were made in Adobe Illustrator by placing a black circle with a 2 pt stroke width and 28 px diameter on a white background. Within this circle was placed another of half the diameter, and a white rectangle was then used to punch out approximately $20 \%$ of the circumference on the right hand side of the inner circle to give the ' $C$ ' shape. This formation was then exported as a $32 \times$ 32 px image file in JPEG format. An image file was also created for the distractor (a $180^{\circ}$ rotation of the target), fixation cross, and blink cross. Once these display elements were prepared, the Python Image Library was used to make each of the 128 stimulus arrangements. The fixation cross was placed at the centre of a $162 \times 162$ px canvas and the items were randomly allocated to 8 predefined positions surrounding the cross. For each arrangement, target and distractor locations were chosen randomly from the 8 positions surrounding the fixation cross, but the target was constrained to appear at each 
location equally often across all of the 128 stimulus arrangements which contained a target.

Stimuli were presented on an LG Flatron F900p monitor with a pixel resolution of $1024 \times 768$ and a vertical refresh rate of $60 \mathrm{~Hz}^{7}$. The screen was viewed from a distance of $60 \mathrm{~cm}$, which was maintained by a chin rest and forehead bar. From this distance, each individual display element subtended approximately $1^{\circ} \times 1^{\circ}$ of visual angle, and the whole search array spanned $3^{\circ} \times 3^{\circ}$. Responses were given using the ' $\mathrm{M}$ ' (for present) and ' $Z$ ' (for absent) keys on a standard computer keyboard. Pupil size of the right eye was recorded with an EyeLink 1000 (SR Research, Ontario, CA) system sampling at $500 \mathrm{~Hz}$, and the experiment was programmed in Experiment Builder (SR Research, Ontario, CA). Eye level and camera position remained constant throughout the recording session for each participant.

\section{Procedure}

Participants were instructed to search for forward facing ' $\mathrm{C}$ 's in the briefly presented displays and respond, as quickly and accurately as possible, as to whether a target was present in the array. Prior to the start of the experiment participants were shown examples of each condition along with the correct response. They were told to press the ' $M$ ' key if they thought a target was present, and the ' $Z$ ' key if they thought it was absent. A four-point calibration was performed for the eye tracker at the beginning of the experiment and further calibrations were performed as required between blocks. Each trial began with a drift check at the centre of the screen. A fixation cross then appeared for $1000 \mathrm{~ms}$, after which the search stimulus was displayed for $250 \mathrm{~ms}$. Participants then had to make their response. After $2000 \mathrm{~ms}$, the fixation cross turned red for 1000 ms signalling an 'optimum blink period', where participants were instructed that they could blink without compromising data quality. A schematic illustration of this trial sequence is presented in Figure 4.2.

\section{Data processing and statistical analysis}

Pupillometry data were preprocessed using the methods outlined in Chapter 3: Eyeblinks were reconstructed with linear interpolation and the data were smoothed with a third-order Butterworth filter (4 Hz cut-off). The average amount of data replaced by blink interpolation across all participants that were included in the analysis was $11.2 \%$. Segments of pupil data $3500 \mathrm{~ms}$ in length were extracted for each trial, time-locked to

\footnotetext{
${ }^{7}$ Note that this display hardware differs from that depicted in Figure 3.2, which was used for all other experiments in this thesis.
} 


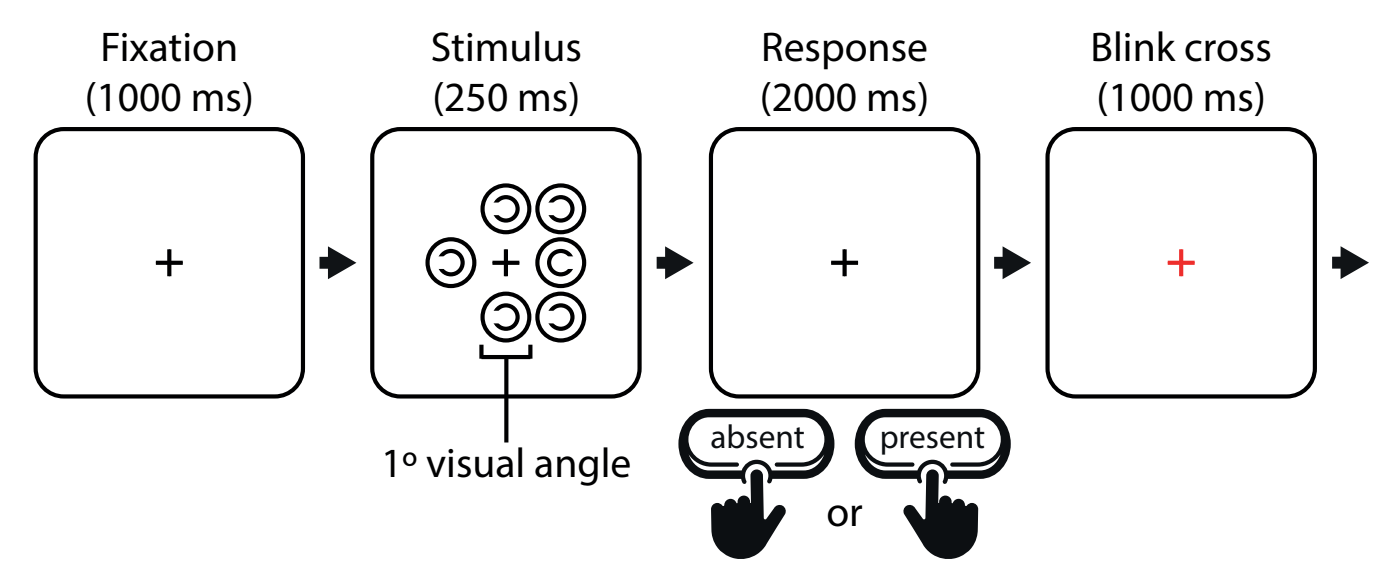

Figure 4.2. Schematic diagram illustrating the trial sequence for Experiment 1. Each trial began with a $1000 \mathrm{~ms}$ fixation. The search array then appeared for $250 \mathrm{~ms}$ and was followed by another $2000 \mathrm{~ms}$ fixation period wherein participants responded 'absent' or 'present'. Finally, the fixation cross turned red for $1000 \mathrm{~ms}$, signalling an 'optimum blink period', where participants were informed they could blink without compromising data quality. Drift corrections and, if necessary, recalibration, took place before the initial fixation.

stimulus onset. These data were expressed as \%-modulation from a baseline calculated as the average pupil size in the $500 \mathrm{~ms}$ period prior to stimulus onset.

The statistical analysis of accuracy, RT and average \%-modulation of pupil size was achieved using two-factor (Target $\times$ Set Size) repeated measures ANOVA. For RT and pupil data, analysis was restricted to correct-only trials. The pupil waveforms were also subject to analysis with nonparametric permutation tests to assess significant modulation from baseline. Because the task resembled a signal detection task, where a signal is either present or absent and an observer responds 'yes' or 'no', the measures $d^{\prime}$ and $c$ were calculated to examine the effect of Set Size on perceptual sensitivity and response bias.

\section{Trial and participant exclusions}

Individual trials were excluded from statistical analysis if (1) a blink event occurred at any time during the $250 \mathrm{~ms}$ stimulus presentation, (2) RT was above or below 2.5 median absolute deviations (MAD) from the participant median (Leys, Klein, Bernard, $\&$ Licata, 2013), (3) average gaze coordinates ( $x$ or $y$ ) across the whole epoch deviated from the fixation cross by $3^{\circ}$ or more, and (4) more than $50 \%$ of pupil data across the epoch had been interpolated. Overall, this lead to the exclusion of 2037 out of 7680 
trials $(26.5 \%)$. The average number of trials excluded for each participant was 67.9 (SD $=74.1)$.

Participants were excluded from further analysis if (1) their number of trial exclusions was above 2 MADs from the median of all participants $(n=6)$, or (2) their proportion of correct responses was below 2 MADs from the median of all participants $(n=2)$. This lead to the exclusion of 7 participants. It is noteworthy that participant exclusion did not alter the general pattern of results.

\subsubsection{Results}

\section{Performance data}

All participants included in the analysis achieved at least $62 \%$ correct responses overall, with the average across all participants and conditions being $78.9 \%(S D=6.9 \%)$. An ANOVA on the accuracy rates revealed significant main effects of both Target, $F(1,22)$ $=4.75, p=.040, \eta_{p}^{2}=0.18$, and Set Size, $F(1,22)=13.76, p=.001, \eta_{p}^{2}=0.38$, with accuracy being higher for target-present trials $(M=83.3 \% \mathrm{~ms}, S D=8.6 \% \mathrm{~ms})$ than for target-absent trials $(M=75.7 \%, S D=13.7 \%)$, and for set sizes of $4(M=81.6 \%, S D=$ $11.2 \%)$ than for set sizes of $6(M=77.3 \%, S D=12.5 \%)$. These findings, displayed on the top row of Figure 4.3, suggest that more effort was required for searches with the larger set size and where no target was present.

Average RT across all participants and conditions was $876 \mathrm{~ms}(S D=159 \mathrm{~ms})$. Again, ANOVA revealed significant main effects of both Target, $F(1,22)=10.10, p$ $=.004, \eta_{p}^{2}=0.31$, and Set Size, $F(1,22)=7.64, p=.011, \eta_{p}^{2}=0.26$. These effects went in the opposite direction to those which were observed for accuracy, with RTs being on average faster for target-present trials $(M=830 \mathrm{~ms}, S D=137 \mathrm{~ms})$ than for target-absent trials $(M=923 \mathrm{~ms}, S D=166 \mathrm{~ms})$, and for set sizes of $4(M=864 \mathrm{~ms}$, $S D=164 \mathrm{~ms})$ compared to $6(M=889 \mathrm{~ms}, S D=154 \mathrm{~ms})$. These effects are shown on the middle row of Figure 4.3, and also indicate that search was more effortful when no target was present and when the set size was larger.

\section{Signal detection measures}

Perceptual sensitivity $\left(d^{\prime}\right)$ and response bias $(c)$ were quantified for both levels of Set Size using SDT. Sensitivity to targets was significantly greater for Set Size 4 compared to Set Size 6, $t(22)=3.78, p=.001, d=1.12$, which can be taken as further evidence for the effort-modulating effects of the set size manipulation. On average, response bias was greater for Set Size 4 than for Set Size 6, but the difference was not significant 

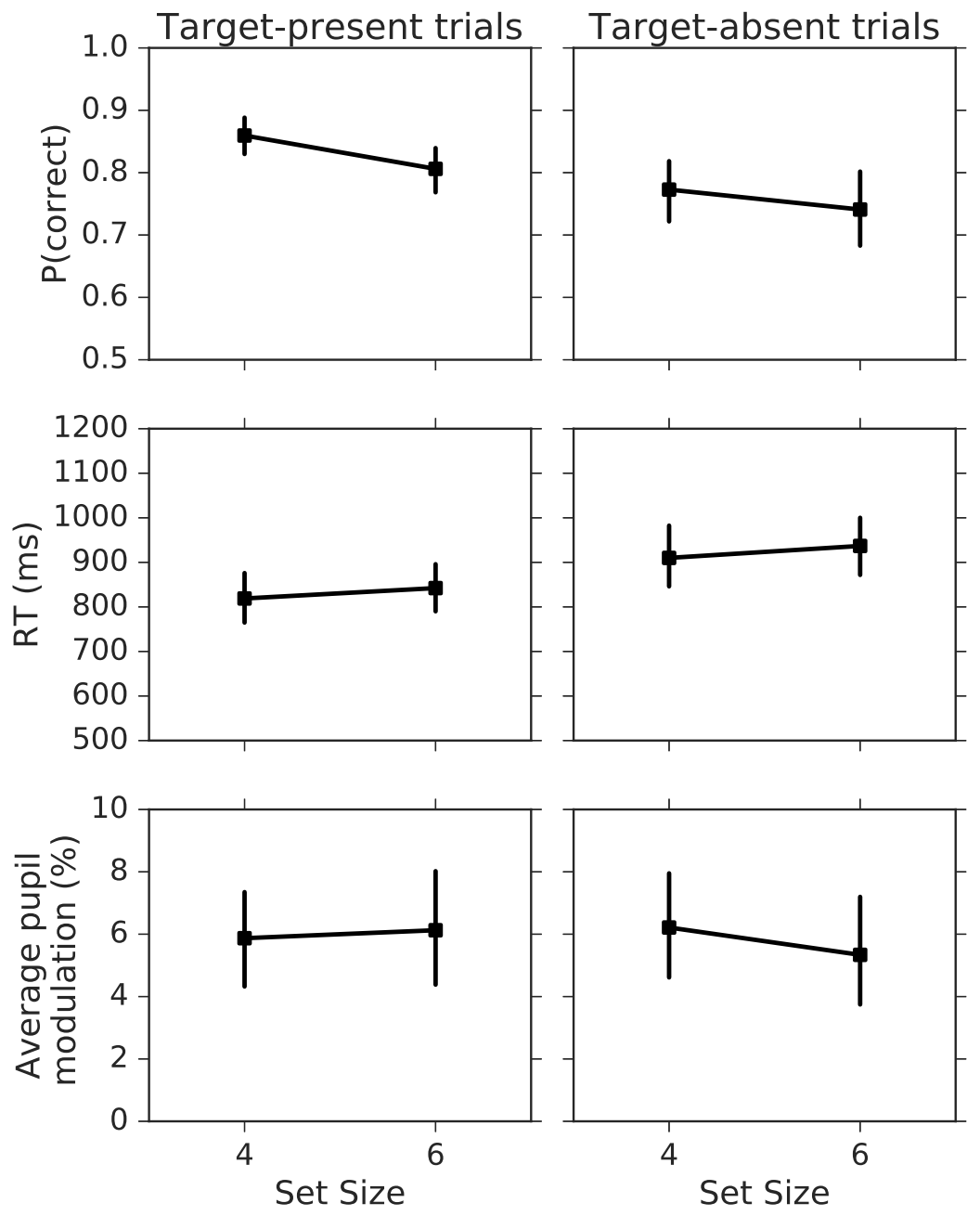

Figure 4.3. Summary of main effects in Experiment 1. Accuracy rates (top), RTs (middle) and average pupil modulations (bottom) are shown for target-present and target-absent trials, and for each level of Set Size. RT and pupil data are shown for correct responses only. Error bars reflect 95\% confidence intervals (bootstrapped, 1000 iterations). 
$(p>.05)$, meaning that the set size difference per se did not bias participants towards responding 'present' or 'absent'. These findings are shown in Figure 4.4.
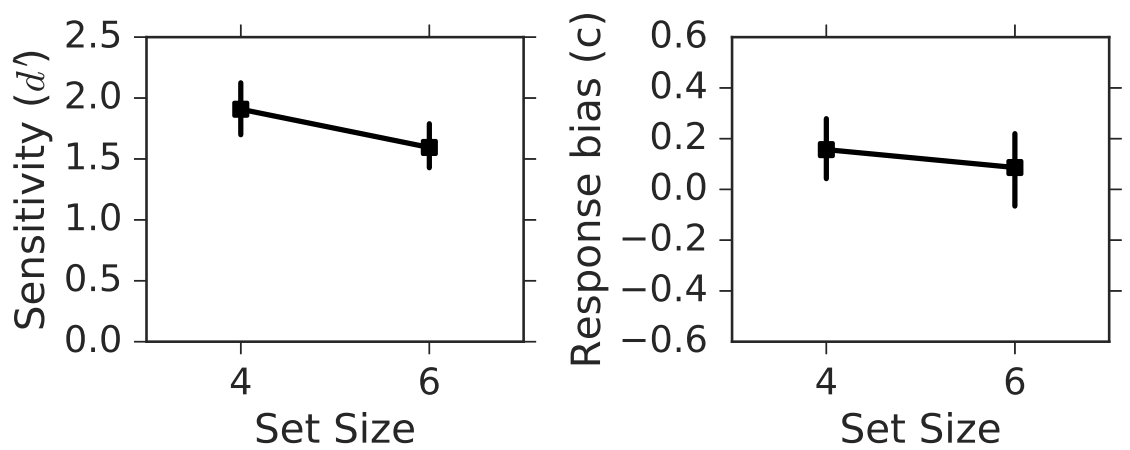

Figure 4.4. Signal detection measures for each level of Set Size in Experiment 2. Sensitivity $\left(d^{\prime}\right)$ was significantly greater for Set Size 4 vs. 6 (left). Response bias (c) was greater on average for Set Size 4 compared to 6, but this difference was not significant (right). Error bars reflect 95\% confidence intervals (bootstrapped, 1000 iterations).

\section{Pupil responses}

Figure 4.5 shows the mean pupil traces for both set sizes on target-present trials (left panel) and target-absent trials (right panel). All conditions elicited roughly the same pattern of pupil dilation. This was characterised by a steady increase in pupil size which, on average, reached peak of $11 \%$ modulation at a latency of 1340 ms poststimulus presentation, and then returned to baseline levels after $3000 \mathrm{~ms}$. Average pupil modulation across all conditions within the $3000 \mathrm{~ms}$ poststimulus period was $5.6 \%$ $(S D=4 \%)$. The temporal extent of significant change from baseline was revealed with cluster-based permutation tests (shown in Figure 4.5). An ANOVA was carried out on the average \%-modulation for the region where all significant clusters overlapped (160 ms prestimulus to $2620 \mathrm{~ms}$ poststimulus). There were no significant main effects of either Target or Set Size $(p>.05)$, but there was a significant interaction, $F(1,22)=$ 4.42, $p=.047, \eta_{p}^{2}=0.17$. Follow-up paired samples $t$-tests revealed that this was due to a Set Size difference on target-absent trials, with average \%-modulation being greater for Set Size 4 compared to set size $6, t(22)=2.30, p=.032, d=-0.68$. This effect is illustrated in Figure 4.6. No other comparisons were significant (all $p \mathrm{~s}>.05$ ). 

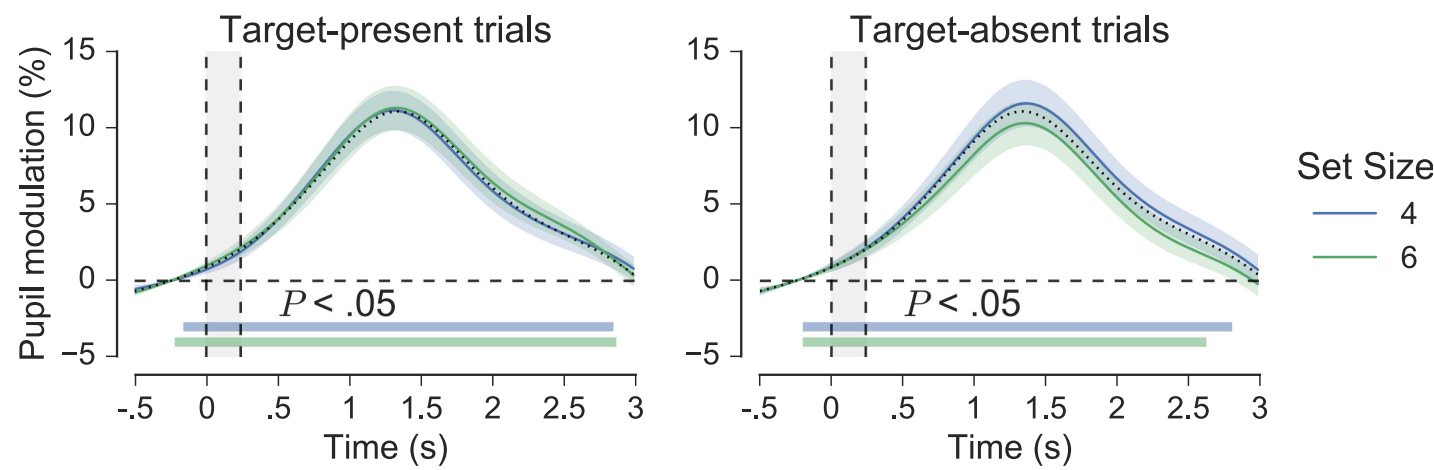

Figure 4.5. Average pupil responses for each trial type in Experiment 1. Stimulus onset occurred at zero for a duration defined by the grey shaded region subtending the vertical dashed lines on each axis. The dotted line on each panel shows the mean pupil trace across all conditions. Shaded areas surrounding each pupil trace show the SEM (bootstrapped, 5000 iterations) and horizontal coloured bars denote periods of significant modulation from baseline, as revealed by nonparametric permutation tests (1024 permutations, $p<.05$, cluster-corrected for multiple comparisons).

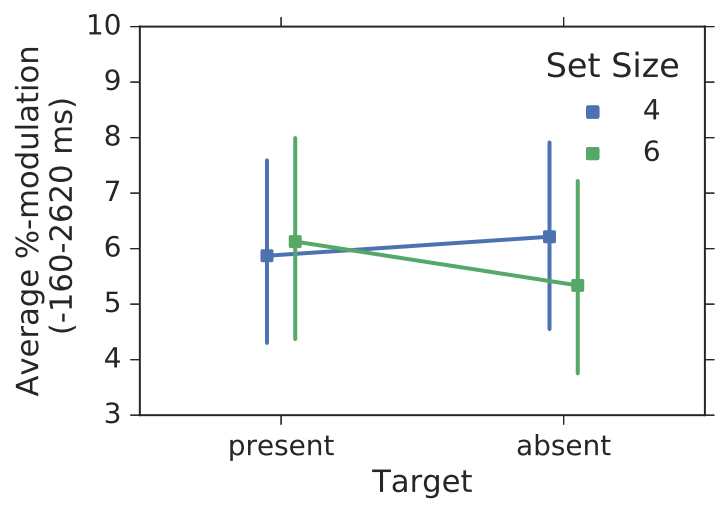

Figure 4.6. The significant interaction between Target and Set Size. Average \%modulation was significantly greater for set size 4 compared to set size 6 on target-absent trials, $t(22)=2.30, p=.032, d=-0.68$. Error bars reflect $95 \%$ confidence intervals (bootstrapped, 1000 iterations). 


\subsubsection{Discussion}

The experiment presented here varied set size and target presence in a visual search task to simultaneously examine the effects of effort and target detection on pupil size. Spatially compact search arrays were presented briefly close to the point of fixation and participants were asked to refrain from making eye movements during experimental trials to remove the possibility that pupil data would be distorted by foreshortening of the camera image and the error that this would introduce to the data. It was predicted that larger set sizes would lead to reduced accuracy, longer RTs and larger pupil responses, and that pupil dilation would generally be larger for target-present compared to target-absent trials.

The performance data indicated that participants detected targets accurately and that searches with the larger set size did indeed require more effort than the smaller set size, with RTs being significantly longer on average for the larger set sizes and perceptual sensitivity $\left(d^{\prime}\right)$ to targets being lower. These effects were not reflected in the pupil data, however, which showed a significant overall response to the stimuli, but little variation between conditions. A significant interaction was observed between Set Size and Target, and follow-up tests revealed this was due to a Set Size difference on target-absent trials, whereby average \%-modulation was significantly greater for Set Size 4 compared to Set Size 6 (Figure 4.6). This finding was somewhat puzzling, as it runs contrary to the general prediction that larger pupil responses would be observed for larger set sizes due to the increased search effort required. Lower accuracy and longer RTs were observed on target-absent trials for Set Size 6 compared to Set Size 4, suggesting that this condition was the more difficult of the two, however this is not reflected in the pupil data.

The general lack of variability in the pupil data with respect to the experimental manipulations despite significant modulation as a result of stimulus exposure suggests that certain aspects of design may have masked any underlying effects. In this experiment, search arrays consisted of black display elements which appeared briefly on a white background close to the point of fixation, constituting a sudden luminance decrement which will have caused the pupil to dilate (Loewenfeld, 1993). In addition to this, participants had to determine whether a target was present or absent for each trial and make speeded button-press responses to indicate their decision, factors which are also known to contribute to pupil dilation (e.g. Einhäuser et al., 2010; Porter et al., 2007; Privitera et al., 2010; Richer \& Beatty, 1985). When designing the experiment it was deemed that these effects would be consistent across trials (with some small variability caused by differences in luminance between the two set sizes), and therefore that any 
effects of effort and target detection would be discernable. However, faith in this assumption may have been misplaced. Given the diminutive nature of the cognitive effects being investigated (the effects of effort and target detection on pupil size described in Sections 4.1.1 and 4.1.2 were typically on the order of $0.5-2 \%$ signal change), the additive nature of the pupillary response (Hoeks \& Levelt, 1993), and the confounding aspects of design mentioned above, it seems likely that any effects of effort and target detection were concealed within the large waveforms observed in this experiment, which could have been driven primarily by the luminance changes associated with stimulus onset, together with the decision-response component of the task. Finally, it is also possible that restricting eye blinks to a specific period, as participants were instructed to do in this task, may itself have been effortful and thereby confounded the pupil responses.

Therefore, to discover the effects of effort and target detection in visual search with brief displays it would appear that a great deal more of attention must be devoted to stimulus and task design, so as to ensure as far as possible that the data are not affected by the decision-response components of the task or luminance artefacts associated with stimulus appearance.

\subsection{Experiment 2}

\subsubsection{Introduction}

The purpose of Experiment 2 was to further investigate the effects of effort and target detection on pupil size in visual search with brief displays by responding to methodological issues which may have compromised the intended effect of manipulations in the previous experiment. Specifically, the aim was to exert greater control over stimulus luminance and the decision-response component of the search task, effectively removing these factors from the poststimulus period, where experimental effects are most likely to be observed. Control over luminance was achieved by changing the stimuli from Landolt 'C's to low-contrast sinusoidal luminance modulations-also known as 'gabor patches' - of varying orientations, whose appearance on a uniform background would not cause any changes in the overall luminance of the display. Gabor patches have been used for this reason in a number of previous pupillometry studies (e.g. see Kloosterman et al., 2015; Privitera et al., 2014), and they have also been used in visual search experiments with brief stimulus displays (e.g. Corbett \& Smith, 2017; Smith \& Sewell, 2013; Smith, Sewell, \& Lilburn, 2015). Control over the decision-response aspect of the experiment was achieved by removing the requirement for a response on half of 
the trials and delaying the response, when it was required, until $2500 \mathrm{~ms}$ after stimulus appearance. These measures should ensure that smaller pupil responses are observed in the poststimulus period overall, and that any variation between conditions be more readily attributable to the cognitive consequences of the experimental manipulations. Given that the key experimental manipulations in this experiment are unchanged, the predictions remained the same (see Section 4.2.1).

\subsubsection{Method}

\section{Participants}

Twenty-six participants completed this experiment but one of them was excluded from data analysis (see below in Section 4.3.2). Of the 25 that remained, age ranged from 19-36 years $(M=21.61, S D=4.83)$ and 17 were female. All participants were students at Swansea University reporting normal or corrected-to-normal acuity and colour vision. Participation was voluntary or in exchange for course credit. The experimental protocol was approved by the Ministry of Defence Research Ethics Committee and the Department of Psychology Ethics Committee at Swansea University. Written informed consent was obtained from each participant.

\section{Design}

The same task design and experimental manipulations were used as for Experiment 1, but with some changes to stimuli and participant interaction. Instead of searching for Landolt 'C's on a white background, participants searched for tilted gabor patches in arrays of horizontal and vertical gabor patches on a grey background. The stimulus arrangements were presented for $200 \mathrm{~ms}$ within $2^{\circ}$ of the central fixation cross. A slightly shorter duration of exposure was chosen for this experiment to further limit the possibility of eye movements, and also because a previous report with similar stimuli and task design (Sewell, Lilburn, \& Smith, 2014) suggested that $200 \mathrm{~ms}$ exposure would result in accuracy rates comparable to those that were observed in Experiment 1 of the current chapter. Example search arrays for each condition are shown in Figure 4.7. Participants completed 512 experimental trials in a single testing session lasting roughly $1 \mathrm{hr}$ (a separate practice block containing 16 trials was completed at the beginning). These were organised into 8 blocks of 66 , each containing approximately equal numbers of all of the experimental conditions exemplified in Figure 4.7. Participants were only asked to indicate whether a target was present on half of the trials, and responses (when required) were made only after a period of $2500 \mathrm{~ms}$ from stimulus presentation had 
elapsed. The cue for participants to make a response was given visually (see later). The number of trials requiring a detection response was also approximately equal for each block. Stimuli arrangements were generated 'on the fly' by the experimental program (more details in Section 4.3.2), with all conditions being randomised for each block. Continuous recordings of gaze position and pupil data were obtained for each trial, and on trials where responses were made, accuracy and RT (from the appearance of the cue until the response, in milliseconds) also. The task was performed in a dimly lit room.

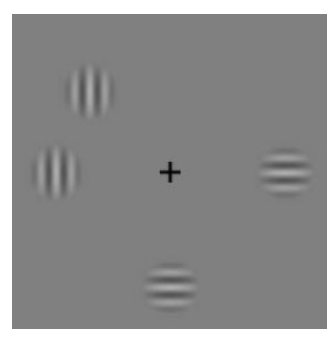

Set Size: 4

Target: absent

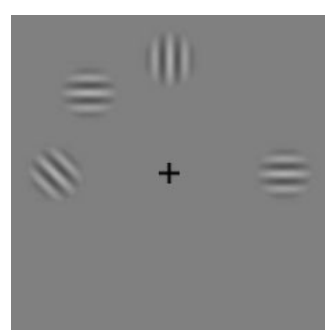

Set Size: 4

Target: present

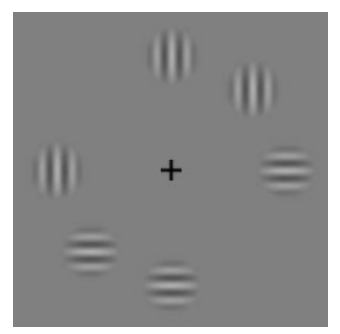

Set Size: 6

Target: absent

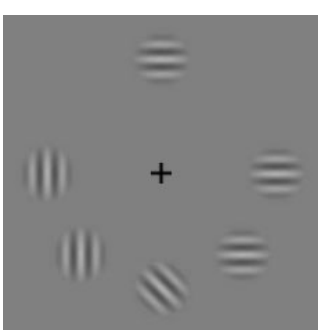

Set Size: 6

Target: present

Figure 4.7. Example stimuli from the four conditions in Experiment 2. The target was a gabor patch with a $45^{\circ}$ tilt, and distractors were horizontal or vertical patches in equal measure (except for target present trials where one of the distractors was replaced by the target). All patches were allocated randomly to one of eight equidistant locations on the circumference of a circle surrounding the fixation cross.

\section{Stimuli and apparatus}

Stimuli in this experiment were gabor patches, enveloped within a cosine window (spatial frequency $=0.1, S D=12,39 \%$ contrast) and presented in sets of 4 or 6 at 8 equidistant locations on the perimeter of a virtual circle $\left(5^{\circ} \times 5^{\circ}\right.$ of visual angle in diameter) surrounding a central fixation cross. The individual gabor patch images were generated online at https://www.cogsci.nl/gabor-generator and saved in JPEG format. They were arranged into search arrays by the experimental program. Trials without targets consisted of horizontal and vertical patches in equal measure, randomly allocated to one of the 8 locations. In trials with targets, one of the distractor patches was randomly selected and replaced with a patch tilted $45^{\circ}$ either to the left or the right, the direction of tilt also being random. The target appeared equally often at all locations across the whole experiment. Each patch spanned roughly $1^{\circ}$ of visual angle.

Stimuli were presented on a 24 in. Ilyama monitor with the pixel resolution set to $1024 \times 768$ (dot-by-dot) at the maximum refresh rate of $144 \mathrm{~Hz}$. The surface 
luminance of the grey background was recorded as $73.56 \mathrm{~cd} / \mathrm{m}^{2}$, and the surrounding dark light of the unused portion of screen as $0.54 \mathrm{~cd} / \mathrm{m}^{2}$ (recorded with a ColorCAL MKII colorimeter, Cambridge Research Systems). The screen was viewed from a distance of $50 \mathrm{~cm}$, which was maintained by a chin rest and forehead bar. From this distance, the individual gabor patches subtended approximately $1^{\circ} \times 1^{\circ}$ of visual angle, with the diameter of the search array spanning a maximum of $5^{\circ} \times 5^{\circ}$. Pupil size of the left eye was recorded with an EyeLink 1000 (SR Research, Ontario, CA) system sampling at $1000 \mathrm{~Hz}$, and the experiment was programmed in Experiment Builder (SR Research, Ontario, CA). Eye level and camera position remained constant throughout the recording session for each participant.

\section{Procedure}

Participants were instructed to search for tilted gabor patches in the briefly presented displays and to indicate, as quickly and accurately as possible, whether a target was present or absent by pressing the ' $\mathrm{M}$ ' key (for present) or the ' $\mathrm{Z}$ ' key (for absent) on the computer keyboard. Prior to the start of the experiment, participants were shown examples of each condition along with its correct response, and it was explained that they should only respond when cued to do so by the appearance of a question mark at the point of fixation. A five-point calibration was performed at the start of the experiment and in between blocks when required. Each trial began with a variable length fixation cross (500-1500 ms), to avoid the possibility that participants would accurately anticipate the appearance of the search display and any effects that this may have on the pupil data. The stimuli then appeared for $200 \mathrm{~ms}$, and this was followed by another $2500 \mathrm{~ms}$ period of fixation. On trials where a response was required, the central fixation cross was then replaced with a question mark of similar size, which cued the participant to indicate whether the target was present or absent by pressing the appropriate response key. When a response was detected, the response cue was replaced by a fixation cross which remained in position for a further $2500 \mathrm{~ms}$. If a button-press was detected at any time other than when the response cue was on the screen, the fixation cross turned red and the words "Bad Trial: only respond when the '?' appears" were shown beneath. This message remained in place for $2000 \mathrm{~ms}$, after which the next trial commenced. This trial sequence is schematically illustrated in Figure 4.8. In this experiment participants were not asked to restrict eye blinks to a specific period of time. 


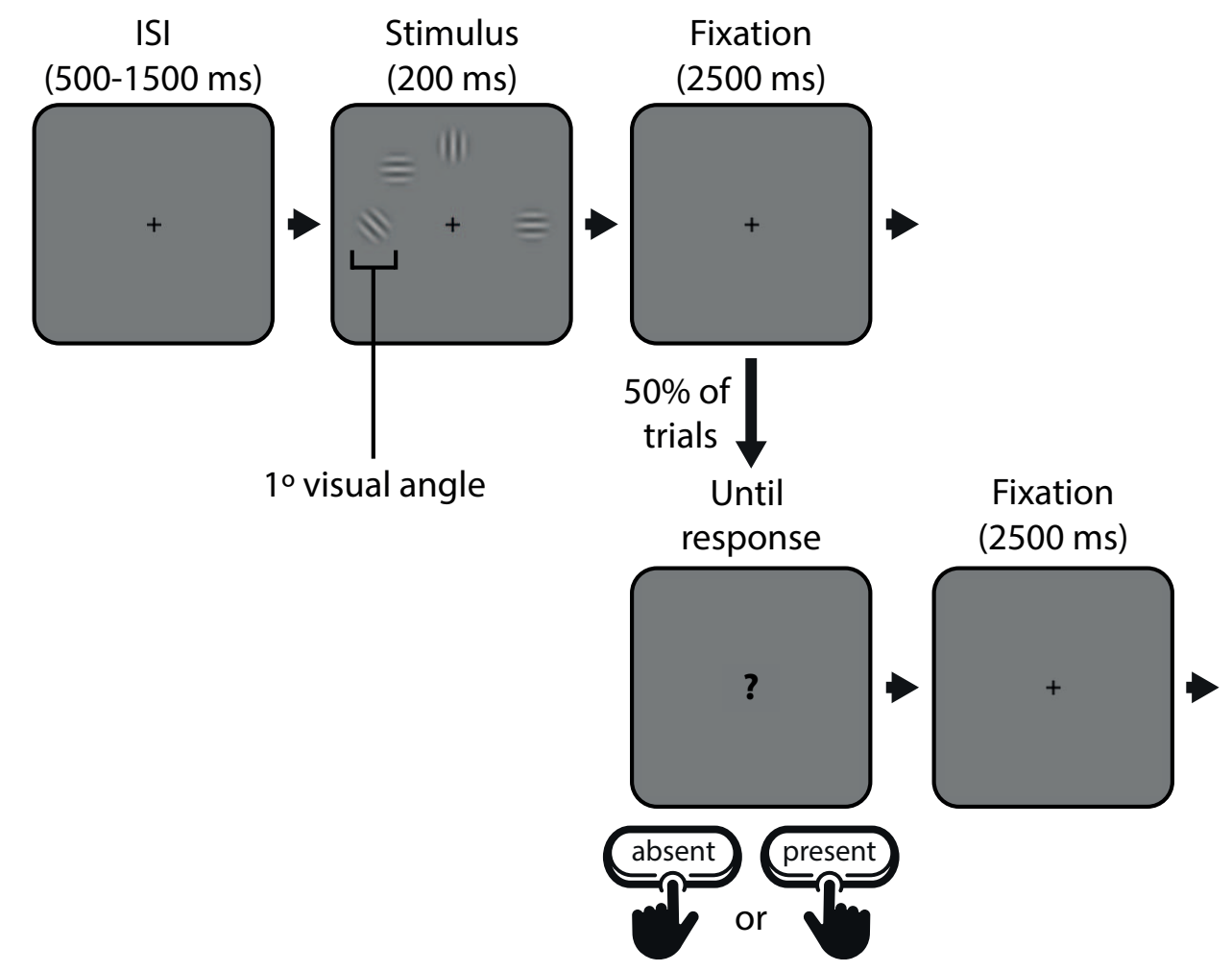

Figure 4.8. Schematic illustration of the trial sequence for Experiment 2. Each trial began with a 500-1500 ms fixation. The stimulus then appeared for $200 \mathrm{~ms}$, and was followed by another $2500 \mathrm{~ms}$ fixation. On trials were a detection response was not required, the trial cycle began anew from this point. When a detection response was required, the fixation cross became a question mark, which remained in place until participants responded 'absent' or 'present'. This was followed by another $2500 \mathrm{~ms}$ fixation, after which the trial sequence was renewed. Drift corrections and, if necessary, recalibration, took place before the initial fixation. 


\section{Data processing and statistical analysis}

Pupil data were processed in a similar fashion to Experiment 1. Eye-blinks were reconstructed with linear interpolation and the data were smoothed with a third-order Butterworth filter ( $4 \mathrm{~Hz}$ cut-off). The average amount of data replaced by interpolation was $22.6 \%$ for all participants that were included in the analysis. Segments of pupil data $2500 \mathrm{~ms}$ in length were extracted for each trial, time-locked to stimulus onset. On trials with detection responses, further segments were extracted, time-locked to the appearance of the response cue. The data were expressed as \%-modulation from a baseline, calculated as the average pupil size in the $500 \mathrm{~ms}$ period prior to stimulus (or response cue) onset.

The pupil waveforms were subject to statistical analysis with cluster-based nonparametric permutation tests to assess the significance of modulation from baseline. Averages for the pupil data were then calculated within the region where all significant clusters overlapped, separately for the stimulus and detection response waveforms. Statistical analysis of RT data and the average \%-modulation data was achieved using two-factor (Target $\times$ Set Size) repeated-measures ANOVA. As with Experiment 1, the signal detection measures $d^{\prime}$ and $c$ were calculated to examine differences in sensitivity and response bias between the two set sizes. For RT and pupil data, analysis was restricted to correct-only trials.

\section{Trial and participant exclusions}

Individual trials were excluded from statistical analysis if (1) a blink event occurred at any time during the $200 \mathrm{~ms}$ stimulus presentation, (2) a button press was detected at any time other than when the question mark was on the screen (in trials where a detection response was made), (3) RT was above or below 2.5 MADs from the participant median, (4) average gaze coordinates ( $x$ or $y$ ) across the stimulus or response epoch deviated from the fixation cross by $3.5^{\circ}$ or more, and (5) more than $50 \%$ of pupil data across the stimulus or response epoch had been interpolated. Overall, this lead to the exclusion of 2232 out of 13728 trials (16.3\%). The average number of trials excluded for each participant was 85.8, with a standard deviation of 71.2. One participant was excluded from the analysis for their number of trial exclusions being 2.5 MADs above the median of all participants (this did not change the overall pattern of findings). 


\subsubsection{Results}

\section{Performance data}

All participants included in the analysis attained at least $64.9 \%$ correct responses, with the average across all participants and conditions being $89 \%(S D=10.2 \%)$. An ANOVA on the accuracy rates revealed significant main effects of both Target, $F(1,22)=12.22$, $p=.002, \eta_{p}^{2}=0.34$, and Set Size, $F(1,22)=5.34, p=.03, \eta_{p}^{2}=0.18$, with accuracy being higher on target-present $(M=96 \%, S D=5.8 \%)$ compared to target-absent $(M=$ $83.1 \%, S D=19.2 \%)$ trials, and for Set Size $4(M=90.6 \%, S D=13.5 \%)$ compared to Set Size $6(M=88.7 \%, S D=197.4 \%)$. Like the corresponding analyses in the previous experiment, these results can also be taken as an indication that Target and Set Size affected the difficulty of the search. No significant interaction was observed.

The average RT across all conditions was $607 \mathrm{~ms}(S D=188 \mathrm{~ms})$. ANOVA revealed a significant main effect of Target, $F(1,22)=14.67, p=.001, \eta_{p}^{2}=0.38$, whereby RT was longer for target-absent $(M=644 \mathrm{~ms}, S D=201 \mathrm{~ms})$ than for targetpresent trials $(M=570 \mathrm{~ms}, S D=168 \mathrm{~ms})$. The effect of Set Size was not significant $(p>.05)$, which does not support the notion that larger set sizes made the search more difficult in this experiment. The interaction between Target and Set Size was not significant. These performance data are summarised on the top (accuracy) and middle (RT) rows of Figure 4.9.

\section{Signal detection measures}

There were no significant differences in perceptual sensitivity $\left(d^{\prime}\right)$ or response bias $(c)$ between Set Size 4 and Set Size 6 ( $p>$.05: Figure 4.4).

\section{Pupil responses}

The mean pupil traces for all conditions are shown in Figure 4.11. As with Experiment 1 , all conditions elicited very similar patterns of dilation. The group-level average pupil responses to stimuli (top row of Figure 4.11) were defined by an average peak dilation of $2.6 \%$ modulation at a latency of $1180 \mathrm{~ms}$ poststimulus onset, and a recovery to baseline levels after approximately $1800 \mathrm{~ms}$. Average pupil modulation in the $2500 \mathrm{~ms}$ stimulus period across all conditions was $0.58 \%$. The permutation tests revealed this general effect of stimulus exposure to be significant for all conditions $(p<.05$ : Figure 4.11, top row). ANOVA was conducted on the averages calculated for the region where all significant clusters overlapped (from 600 to $1480 \mathrm{~ms}$ in the poststimulus period), but 

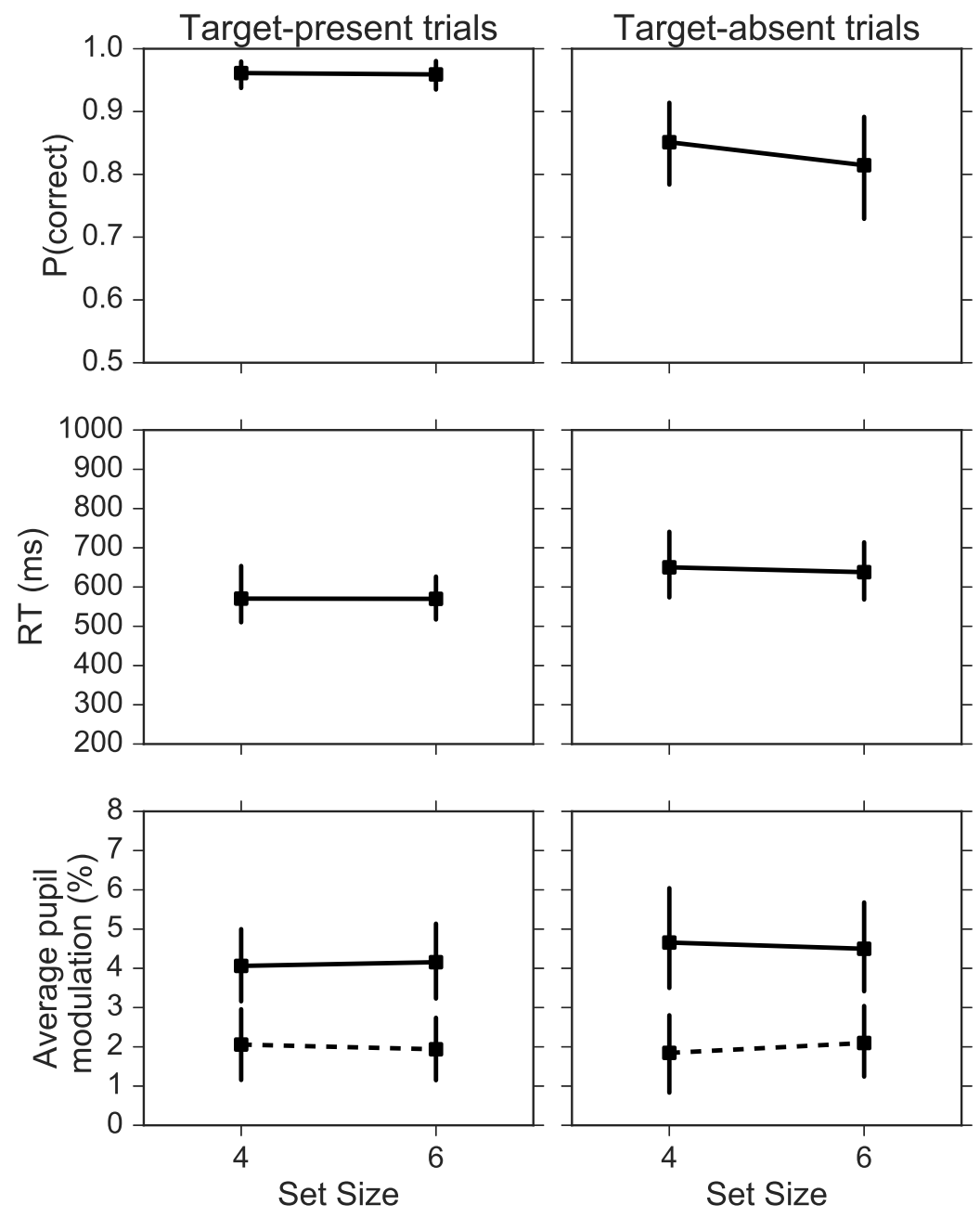

Figure 4.9. Summary of main effects in Experiment 2. Accuracy rates (top), RTs (middle) and average \%-modulation of pupil size (bottom) are shown for target-present and target-absent trials, and for each level of Set Size. RT and pupil data are shown for correct responses only. On the graphs showing pupil responses, the solid lines show the average \%-modulation following the response cue and the dashed lines show the average \%-modulation following stimulus presentation. Error bars reflect $95 \%$ confidence intervals (bootstrapped, 1000 iterations). 

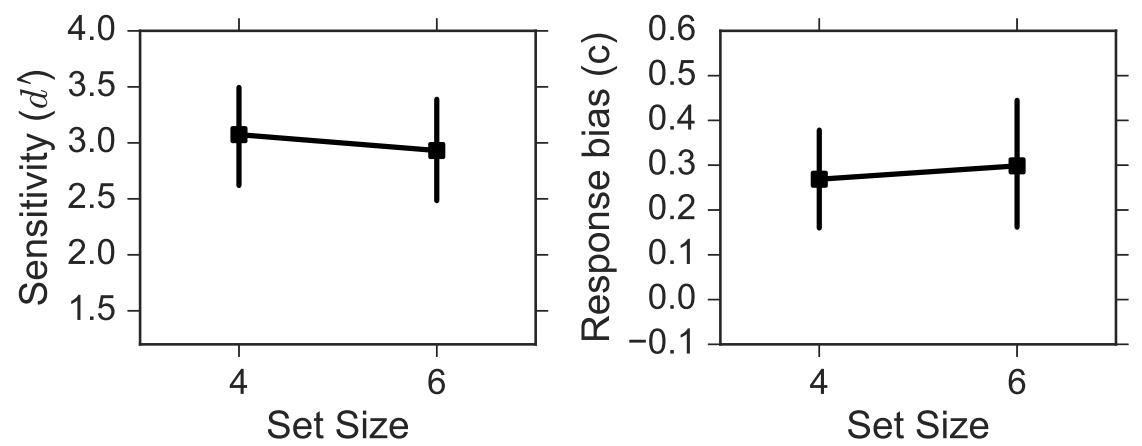

Figure 4.10. Signal detection measures for each level of Set Size in Experiment 2. Sensitivity $\left(d^{\prime}\right)$ was significantly greater for Set Size 4 vs. Set Size 6 (left). Response bias (c) did not differ significantly between Set Sizes. Error bars reflect 95\% confidence intervals (bootstrapped, 1000 iterations).

neither Target nor Set Size had a significant effect ( $p>.05$ : bottom row of Figure 4.9, dashed lines).

The pupil responses during the behavioural response periods (bottom row of Figure 4.11) were also subject to analysis. These were larger than the responses to stimuli, with a peak dilation of $3.27 \%$ modulation at a latency of $1320 \mathrm{~ms}$, approaching baseline by the end of the $2500 \mathrm{~ms}$ period. Average pupil modulation for the detection responses was $4.34 \%$. The permutation testing revealed the effect of a detection response to be significant in all conditions ( $p<.05$ : Figure 4.11, bottom row). The region defined by the overlap of all significant clusters was between 260 and $2500 \mathrm{~ms}$ in the poststimulus period. ANOVA did not reveal any significant effects of Target or Set Size on the average pupil data within this region $(p>.05$ : bottom row of Figure 4.9, solid lines).

\subsubsection{Discussion}

This experiment addressed methodological issues arising from the previous experiment that were thought to have confounded the observed pupillary responses. Most notably, low-contrast gabor patches were used as the search stimuli instead of Landolt 'C's, and the decision-response component of the task was delayed by $2500 \mathrm{~ms}$. The former of these alterations aimed to minimise the extent to which stimulus appearance would invoke the PLR, and the latter aimed to remove the effects of decision making and buttonpressing from the poststimulus period. Together it was thought that this would help to isolate cognitive effects on the pupil arising from the experimental manipulations. 

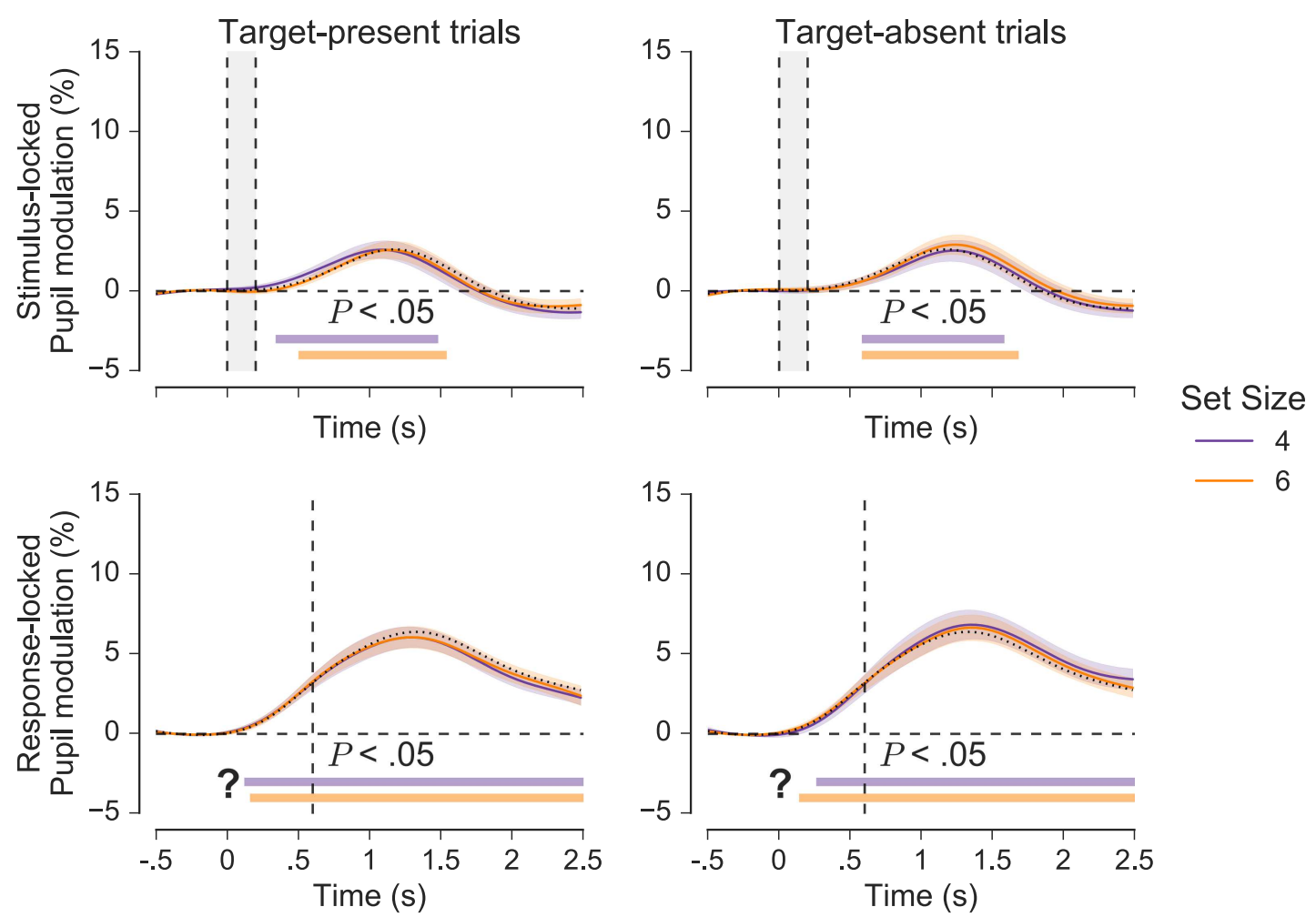

Figure 4.11. Average pupil responses for each trial type in Experiment 2, with $y$-axis at same scale as for Experiment 1. The top row shows pupil responses following stimulus presentation, which occurred at zero for $200 \mathrm{~ms}$ (shown as the grey shaded region subtending the vertical dashed lines on each axis). The bottom row shows pupil responses following the response cue, which occurred at zero. The dotted trace on each panel shows the mean pupil trace across all conditions, and the dashed vertical line on each of the bottom panels shows the average RT across all conditions. Shaded areas surrounding each pupil trace reflect the SEM (bootstrapped, 5000 iterations) and horizontal coloured bars denote clusters of significant modulation from baseline, as revealed by nonparametric permutation tests (1024 permutations, $p<.05$, clustercorrected for multiple comparisons). 
As with Experiment 1, it was predicted that larger set sizes would lead to worse performance measures and larger pupil responses, and that pupil dilation would be larger for target-present compared to target-absent trials.

Once again, the pupil data still showed little variability with respect to Target and Set Size. There was however significant modulation from baseline in the poststimulus period, despite there being no change in overall luminance at the time of stimulus exposure. These observed responses must therefore have been caused by some physical property of the stimuli other than luminance, certain aspects of cognition linked to task performance, or a combination of both. Regarding the physical properties of the stimuli, the spatial frequency component of the gabor patches could have been causing the pupil to dilate (e.g. Barbur et al., 1992; Barbur et al., 1998; Goldwater, 1972; Woodhouse, 1975). However, if this was the case, one would expect to see bigger responses on average for the larger of the two set sizes, because arrays of 6 gabor patches contain precisely one third more spatial frequency information than arrays of 4 . Therefore the observed responses following stimulus exposure are most likely a reflection of the cognitive processes involved in the task. The lack of variability in pupil dilation with respect to Target and Set Size does not preclude the possibility that target detection and effort still contributed to the observed responses in some way. It could be that these effects were present in some capacity on every trial. For instance, Set Size appears to have caused no changes in effort, or at least none that can be ascertained from the RT data, but each trial may have required a similar level of cognitive effort which caused the pupil to dilate. Similarly, although participants were instructed to search for tilted gabor patches, the correct recognition of arrays without targets was also an important aspect of task fulfilment. This means that both target-present and target-absent trials may have functioned as integrated target percepts in their own right, each requiring their own response, which would lead to a consistent effect of target detection on every trial.

Another factor which may have contributed to the observed pupil responses is the decision component of the task. Although behavioural responses were only collected on half of the trials with a button-press delayed by $2500 \mathrm{~ms}$ from the appearance of the stimulus, participants most likely made the actual decision over whether to respond 'present' or 'absent' in the poststimulus period in preparation for the response cue, should it appear. This interpretation is consistent with recent studies that have linked pupil dilation to the decision component in forced-choice detection tasks (e.g. de Gee et al., 2017; de Gee et al., 2014; Urai et al., 2017, see also Section 2.5.2).

Finally, the bottom row of Figure 4.11 shows a substantial effect of pupil modulation during the response period. The only display change at this point was the transition 
Pupillometric effects of effort and target detection in visual search

between the fixation cross and the response cue, which were of approximately equal size, suggesting that the effects of luminance can be safely discounted. The large responses observed at this point therefore most likely reflect the effects of response execution, together with any latent decision processes that were not fully formed in the poststimulus period. Richer and Beatty (1985) observed pupil responses of a similar shape and magnitude in a simple detection task, and many other recent studies have reported similar effects of motor activity on pupil dilation (e.g. Einhäuser et al., 2010; Hupé et al., 2009; Kloosterman et al., 2015; Privitera et al., 2010, see also Section 2.5.2).

\subsection{Overall discussion}

Whilst the pupil data in these experiments showed limited significant variability with regards to the experimental manipulations of Target and Set Size, they did help to confirm certain characteristics of the cognitive pupillary response and provide some indication as to the validity for using pupillometry to explore cognitive processes in visual search with brief stimulus displays. In the first experiment, large responses to the stimuli were observed, reflecting the luminance decrement associated with stimulus onset and the effects of the decision-response component of the task that were present for each trial. In the second experiment, the effects of luminance and button-pressing were removed from the poststimulus period and much smaller responses were observed, most likely reflecting the preparatory decision process and the 'detection' of whether the search stimulus did, or did not, contain a target. Additionally, the waveforms during the behavioural response periods for Experiment 2 confirm a substantial effect of buttonpressing, corroborating similar observations from many other pupillometry studies (e.g. de Gee et al., 2014; Einhäuser et al., 2010; Einhäuser et al., 2008; Fahle et al., 2011; Hupé et al., 2009; Kloosterman et al., 2015; Porter et al., 2007; Privitera et al., 2014; Privitera et al., 2010; Richer \& Beatty, 1985; Richer et al., 1983; Smallwood et al., 2011).

The lack of significant main effects of Target and Set Size in both experiments despite the removal of luminance and button-pressing confounds in the second experiment suggests that these manipulations were not sufficient to evoke reliable effects of effort and target detection. Regarding the Set Size manipulation, it could be that the difference in effort required for arrays of 4 and 6 items was negligible, or simply not big enough to evoke observable differences in pupil dilations. Porter et al. (2007) did report larger pupil dilation on average for larger set sizes, but this effect corresponded to a 
larger difference in RTs, and the quantitative difference in set sizes was much larger (a difference of 20 items compared to the difference of 2 items used in these experiments). Set sizes in the current experiment were constrained to such small quantities to ensure that all items could be positioned at a similar distance to the fixation cross during the brief period of exposure, ruling out the possibility that some items would receive preferential processing on account of their proximity to the locus of gaze, and also to avoid pushing VWM too far beyond its known capacity for 4-5 objects (e.g. Luck \& Vogel, 1997; Phillips, 1974; Sperling, 1960; Vogel et al., 2001). Regarding the Target manipulation, what seems clear upon reflection is that the target itself was not the object of search. When participants had to respond, they had to say either that the target was present or absent, meaning that search arrays with and without targets effectively constituted separate targets in themselves, each requiring a different response, and each of them important to the fulfilment of the task. Pursuing this line of reasoning, the significant modulation observed following stimulus exposure in Experiment 2 might be thought of as an effect of target detection where one of two possible targets (i.e. target-present and target-absent search arrays) was present on every single trial. Such an interpretation would be consistent with the adaptive gain theory of LC-NA function (Aston-Jones \& Cohen, 2005), which posits that phasic activation of the LC in response to task relevant stimuli facilitates behavioural exploitation of the environment.

In conclusion, the experiments outlined in this chapter demonstrated cognitive pupil responses to search in the most general terms, but they did not appear to be sensitive to manipulations of target presence and search difficulty. This may be largely attributable to the design constraints that were necessary to remove the confound of pupil foreshortening. Briefly presented search arrays with small set size differences may not be sufficient to push the cognitive system hard enough for any effects to be observed in the pupil, and the forced-choice nature of the task may have undermined the effect of target detection. A more optimal approach might therefore be to use pupillometry to explore search processes in standard search paradigms, allowing eye movements, but adopting countermeasures and / or corrective measures to deal with pupil foreshortening. After all, this would be necessary in any form of practical application, where critical stimuli rarely appear for brief periods around the locus of fixation, and must instead be searched for with eye movements. 



\section{Chapter 5}

\section{Pupillometry with foreshortening correction in free-viewing visual search}

\subsection{General introduction}

The experiments presented in the previous chapter were designed to explore what pupillometry can reveal about the cognitive processes of visual search, specifically those linked to effort and target detection. In both of the experiments, compact search arrays with small set size differences were presented for brief durations close to the point of fixation and participants had to search their VWM representation of the stimulus to determine whether a target was present or absent, indicating their response with a button-press. Brief stimulus presentations and the prohibition of eye movements were precautions taken to ensure that the pupil data were not systematically affected by the pupil foreshortening error (PFE), where changes in gaze position relative to the optical perspective of the camera lead to false estimates of pupil size. These methodological precautions all but eliminated the PFE, but they also imposed design constraints which limited the scope for, and efficacy of, experimental manipulations, and ultimately resulted in paradigms with narrow relevance to the ecology of human visual search behaviour. The present chapter therefore continues the pupillometric investigation into visual search, but with the alternative approach of allowing eye movements and attempting to counter the PFE through aspects of experimental design and with corrective procedures.

To devise a robust methodology that will be fit for the present aims it is necessary to review the relevant methods already established. It has already been expressed that 
the use of pupillometry in free-viewing search paradigms is uncommon. The notable examples of Porter et al. (2007) and Klingner (2010a) were outlined in Section 4 of the previous chapter. Whilst these experiments shared some basic characteristics, they differed markedly with respect to how the pupillometry data were analysed. Porter et al. adopted what might be described as a 'whole-trial' approach, aligning pupil data to stimulus onset and the moment of response, looking at the general pattern of changes between these two anchor points, and collapsing the data to summary statistics for the purpose of analysis. Klingner on the other hand used a novel technique called 'fixation-aligned pupillary response averaging', which involves identifying events of interest from gaze data once the experiment is complete, aligning segments of pupil data to these events, and then averaging. Klingner's whole report was in fact concerned principally with evaluating the validity of this technique, which up to that point had not been established. The results were promising, suggesting that it can help to isolate pupillometric cognitive effects associated with task components which occur at unpredictable times during unstructured visual tasks, but there was limited statistical analysis or discussion of findings with respect to the experimental psychology literature. Each of these approaches to analysing pupillometry data are now discussed with regards to the context within which they were implemented and their suitability for making inferences about cognitive processes in visual search. First however, the PFE will be reviewed in detail, together with three reports that have explored this issue with EyeLink systems and proposed corrective procedures for dealing with it.

\subsubsection{Pupil foreshortening and corrective procedures}

The PFE is caused by perspective distortion arising from changes in eye position relative to the optical axis of the camera. If unaccounted for by a measurement system and not recognised as a problem by its user, the PFE can lead to misinterpretations of pupil data. Gagl et al. (2011) provided an elegant demonstration of the problem within the context of a reading experiment. Using an EyeLink 1000 system, they first quantified the PFE with an artificial eye which was made to mimic the eye movements that would be required for a sentence reading task. Despite having a pupil of fixed size, the changes in horizontal gaze position across $28^{\circ}$ of visual angle caused pupil recordings to vary between $+5 \%$ and $-13 \%$ from baseline. Gagl et al. subsequently observed similar patterns of error in the pupils of real participants as they performed sentence reading and 'Z-string' scanning ${ }^{1}$ tasks requiring the same changes in horizontal gaze position. Pupil size was greater on average for sentence reading, as would be expected based

\footnotetext{
${ }^{1} \mathrm{~A}$ 'Z-string' scanning task is a reading task where the words are composed entirely of the letter Z.
} 
on the differences in cognitive load, but the recordings for both conditions visibly contained the same pattern of error that was observed with the artificial eye. Gagl et al. then evaluated two functions for correcting the error: a physical function based on the known parameters of the experimental layout (i.e., the distance from the artificial eye to the screen, its fixed pupil size and horizontal gaze position relative to the screen), and a synthetic function based on polynomial curve fitting. Both of the functions calculated the measurement error for a given horizontal gaze coordinate and yielded values which could then be used to correct the pupil data obtained from the real participants. When applied to the sentence reading task, both corrective procedures visibly removed the systematic error and left pupil responses which closely matched those observed in previous studies investigating reading with isolated words (e.g Võ et al., 2008). The best results were obtained with correction parameters derived from the synthetic function and the artificial eye data, but similar results were obtained by fitting the synthetic function to the data for the 'Z-string' scanning task. Gagl et al. concluded that an effortless perceptual task with oculomotor demands that are similar to the experimental task might serve as a practical basis for deriving corrective measures when researchers do not have access to an artificial eye model and the equipment required for its precise control.

Gagl et al.'s (2011) demonstration of the PFE and its subsequent correction focused exclusively on horizontal gaze position. In a free-viewing task, such as natural scene viewing or visual search, it would also be necessary to account for the effect of vertical eye movements. Brisson et al. (2013) devised a method for this which was similar in principle to the 'Z-string' scanning task used by Gagl et al. Participants tracked a small blue ellipse as it traced an elliptical pattern counter-clockwise around the screen. Although the stimuli were of a constant luminance and the task was low in arousal and cognitive demand, the pupil data showed clear patterns of sinusoidal variation which mirrored the sine and cosine functions of the movement of the stimulus. The error was quantified with a linear regression which estimated pupil diameter as a function of the horizontal and vertical coordinates of gaze. With the EyeLink 1000 system ${ }^{2}$, this regression explained on average $9.9 \%\left(R^{2}\right)$ of the variance in pupil size. The coefficients from the regression model associated with the $x$ and $y$ gaze coordinates could then be used to correct the pupil data accordingly. This method constitutes a practical solution to correcting the PFE in free-viewing experiments providing the trajectory of the tracking object during the estimation procedure maps the horizontal and vertical limits of the experimental stimuli.

\footnotetext{
${ }^{2}$ Brisson et al. also quantified the error using two other eye trackers.
} 
The most comprehensive method to date for correcting the PFE was provided by Hayes and Petrov (2015), who mapped the error across the full extent of a 21-in. screen using artificial eyes. With the help of laser orientation apparatus and equipment for mechanical calibration, the eyes were made to 'fixate' small black target discs arranged in a grid across the screen. Grid-based error maps were then produced for three artificial eyes with different fixed pupil sizes across three different geometrical layouts, all of which showed systematic variations in pupil size with respect to gaze position. Correction was implemented using a geometric model, where the error was defined as a function of the cosine of the angle between the eye-to-target and eye-tocamera axes. This corrective model decreased the root mean squared error of pupil size measurements by $82.5 \%$ when its parameters were fixed with regards to the physical geometry of the experimental layout, and by $97.5 \%$ when they were optimised to fit the observed error maps. Implementation of this corrective procedure requires exact replication of one of the three experimental layouts used by Hayes and Petrov, or the similar use of an artificial eye model to map the error for one's own layout.

\subsection{2 'Whole-trial' analysis of pupil data}

As explained in the previous chapter, Porter et al. (2007) recorded pupil size as participants searched for target 'C's among distractor 'C's facing other directions, with the aim of gaining insight into the cognitive effort required for search conditions of different difficulty. Button responses were made on every trial to indicate whether, depending on the task and condition, a target was present or absent, 1 or 2 targets were present, or there was an odd or even number of items in the display. All trials therefore involved a 2-alternative forced choice decision process and ended with a button response to communicate the decision. Pupil data between stimulus onset and the moment of response were time-normalised to account for differences in RT between trials and to ensure that averages reflected equivalent types of cognitive processing at all stages of performance. Data across the whole period were then collapsed to averages for the purposes of statistical analysis. The general pattern of pupil responses during task performance, together with the results from the analyses on the averages, confirmed that pupil size increased in accordance with search difficulty (i.e. as set size and distractor heterogeneity increased). The largest and steepest responses however were observed in the counting condition, where accuracy depended on perfect locational memory (to avoid double counting), leading the authors to suggest that effort due to locational memory load may have been the principal driving force behind the pupil responses observed during search. Support for this idea was also derived from the second experiment, 
where the slope of pupil dilation was steeper in a window towards the end of search in the 1- vs. 2-target condition compared to the present-absent condition, suggesting a cognitive component linked to the discovery of the first target and the remembrance of its location.

Overall, Porter et al.'s approach to analysing pupil data yielded valuable insights regarding the general shape of pupil responses in visual search and the effects of search difficulty. The locational memory interpretation also seems plausible in light of the fact that pupil dilation was larger and steeper in the early stages of counting and towards the end of search for 1 vs. 2 targets, where higher demands on locational memory would be expected. The authors note however that the general shape of the pupil responses was not the best basis for inference, because differences in RT - up to around $5 \mathrm{~s}$ on average in the first experiment-will have influenced the extent to which each waveform was stretched or compressed to fit the normalised timescale. Another general observation was that the responses most likely represented a composite of several task processes, such as automatic reflexes, the motor response at the end of every trial, and cognitive components to do with effort, memory load, target detection and decision making. Again, this interpretation is plausible given the range of known cognitive effects on the pupil (note Section 2.5.2), but Porter et al.'s design and method of pupil analysis did not isolate the relative contribution of these individual task processes to the observed responses.

One final issue to consider regarding the pupillometry methods used by Porter et al. (2007), which was touched on briefly in the previous chapter, is if and how the PFE affected their observations. The authors made recordings with a locally developed head-mounted video-based system which measured the horizontal width of the pupil, always at its widest point (Alexandridis, Leendertz, \& Barbur, 1991). Measurements for each participant were then converted from volatages to milimeters using a calibration procedure involving a printed black dot of a known size being placed just in front of the pupil.As the system always recorded horizontal and not the maximum diameter of the pupil ${ }^{3}$, changes in horizontal gaze position will have introduced measurement error. This would have been most problematic if exacerbated by certain regularities in participant viewing behaviour. For instance, if participants systematically 'read' the search arrays from left to right, which one could imagine would be a tempting strategy in the counting condition, where the largest responses were observed. This however can not be verified and remains a matter of speculation, as gaze position was not recorded in these experiments.

\footnotetext{
${ }^{3}$ The major axis of the pupil ellipse would always reflect the true maximum diameter.
} 


\subsubsection{Fixation-aligned pupillary response averaging}

Fixation-aligned pupillary response averaging was proposed by Klingner (2010a) as a technique for exploring changes in cognitive load that occur at unpredictable times during a visual task. In essence, it involves identifying events of interest by examining gaze data, extracting segments of pupil data which coincide with these events, and then temporally aligning and averaging these data together. Klingner illustrated the technique with example analyses for a visual search and a map reading task.

In the visual search task, participants had to count the number of target 'L's that were present in an array of distractor 'T's and report at the end of the trial via mouseclick whether $0,1,2$ or 3 targets were present. The targets were black, presented on a grey background, and masked with ' $\mathrm{X}$ 's prior to search onset to minimise the effects of brightness and contrast transitions on the pupils' reflexes. The gaze events used to select the pupil data were fixations on targets (within $1.25^{\circ}$ ) and fixations away from targets (at least $5^{\circ}$ ), none of which were among the first or last 5 fixations of a trial. Average pupil responses from $2 \mathrm{~s}$ before the fixation to $3 \mathrm{~s}$ after the fixation were calculated for 770 target fixations and 1511 control fixations across 17 participants. Whereas control fixations showed no consistent response, target fixations evoked small transient pupil dilations of the kind observed by Privitera et al. (2010), changing by around $0.06 \mathrm{~mm}$ and peaking around $1 \mathrm{~s}$ after fixation. Subdivision of the target fixations into discoveries $(n=449)$ and revisits $(n=321)$ revealed that the pupil response following revisits began slightly before the fixation, which was interpreted as possible evidence for pupillometric effects of memory processes, as may be associated with recalling the location of a previously visited target or planning a saccade in order to affirm its location.

In the map reading task, participants explored a fabricated map with a range of abstract symbols, each of them indicating the habitat locations of different species of frog or toad. The instructions were to explore the map and look up the symbols in a legend to see which represented frogs and which represented toads. As with the visual search task, the display was low in contrast and did not involve changes in luminance. The gaze events used to select pupil data in this task were scan paths encompassing legend references, characterised as fixation sequences from a cluster of map symbols to the legend, and then back to the same cluster of symbols. The epochs were aligned with respect to the saccades to and from the legend, and time-normalisation was used to transform the pupil data and account for differences in legend reference dwell time. The average pupil response for 925 legend references across 15 participants showed a small dilation of around $0.02 \mathrm{~mm}$ beginning around $1 \mathrm{~s}$ prior to the saccade to the legend, a constriction and recovery of around $0.06 \mathrm{~mm}$ when looking at the legend, and a return 
to the map at somewhat higher pupil size. This pattern of results was speculatively interpreted as a reflection of WM load, which, it was suggested, would increase when committing a map symbol to memory, be released when examining the legend, and finally increase again as the running count of frogs and toads was updated according to the symbol's classification.

Klingner's (2010) report proved that fixation-aligned pupillary response averaging is a valid approach to the analysis of pupillometry data in free-viewing visual tasks. Unlike the standard alternative of time-locking pupil data to stimulus or response events, this method does not provide a continuous measure of task performance. Instead it has the advantage of flexibility, allowing researchers to define their own events of interest within the task. What is more, measures can be taken during event selection to ensure as far as possible that the extracted epochs only contain cognitive effects associated with specific task components. For instance, in his visual search example, Klingner was interested in the effect of target fixation, and therefore ensured that motor responses and display changes would not affect the averages. He did this by discarding the first and last 5 fixations of each trial, and by having participants respond with a mouse click at the end of each trial to indicate how many targets they had found. This meant that the dilation observed in the averaged waveform reflected the attentional processes to do with target detection, as no other task components were consistently present.

Foreshortening may not have been a systematic source of error in Klingner's work due to the use of a Tobii 1750 system for data collection. This system yields millimetre measurements by first recording the largest diameter, in pixels, of an ellipse fitted to the pupil image and then multiplying this value by a scaling factor derived from the recorded interpupillary distance, which accounts for the distance between eye and camera. Although this method may not be affected by pupil foreshortening, it is still affected by small errors arising from differences in iris anatomy and pupil shape (Gagl et al., 2011).

\subsubsection{Summary and current direction}

The research outlined above highlights the methodological challenges associated with designing free-viewing pupillometry experiments that are sufficiently well-controlled so as to allow for cognitive interpretations of the pupillary response. The issue of pupil foreshortening was discussed first, along with three methods that have been proposed for its correction. Precisely controlled artificial eyes provided the best insight into the error and yielded the best corrective functions (Gagl et al., 2011; Hayes \& Petrov, 2015), but data-driven practical alternatives have been implemented to good 
and reliable effect (Brisson et al., 2013; Gagl et al., 2011). Two established approaches for analysing pupil data in visual search were also discussed: whole-trial analysis (Porter et al., 2007), which looks at the general pattern of pupil responses between stimulus onset and the moment of response, and fixation-aligned pupillary response averaging (Klingner, 2010a), which focuses on pupil dynamics surrounding specific task components identified via gaze data.

A more-detailed insight into what causes the pupil to dilate during visual search might be gained from a synthesis of some of the aforementioned methods. For instance, with an appropriate task design, whole-trial analysis and fixation-aligned pupillary response averaging could be used in conjunction with one-another to address complimentary questions about task-relevant cognition, the former giving a general idea about the processing involved across the entire search period and the latter focusing more specifically on processes associated with events occurring at unpredictable times during search. Together with carefully prepared stimuli and a procedure for correcting the PFE, this would constitute a robust methodology for investigating some of the unresolved questions regarding the cognitive pupil dynamics of visual search.

\subsection{Experiment 3}

\subsubsection{Introduction}

The main experiment in this chapter, Experiment 4, uses pupillometry to examine cognitive processes in free-viewing visual search. The full details of Experiment 4 are given in Section 5.3, but to summarise briefly, participants completed 152 trials of multiple-target search across two separate experimental sessions. The search arrays spanned $20^{\circ} \times 20^{\circ}$ of visual angle and were displayed for up to $10 \mathrm{~s}$, meaning that every trial would contain eye movements and therefore that pupil size measurements would be affected by the PFE. Precautions were taken against this issue at the level of stimulus design by ensuring the random positioning of display elements, but eye movements would still add noise to the pupil data and the error could be made systematic throughout trials by regularities in participant viewing behaviour. Experiment 3 was therefore conducted in conjunction with Experiment 4 to gain a general understanding of how pupil size varied with respect to gaze position in the visual search task for each participant recording session and to devise corrective measures for potential application to the pupil data. This was achieved using an adaptation of the ellipse tracking task implemented by Brisson et al. (2013), which was outlined in Section 5.1.1. Participants were asked to track a small blue ellipse as it traced a circular pattern counter-clockwise 
around the screen. Crucially, the path of the ellipse subtended the horizontal and vertical limits of the portion of the screen where stimuli could appear in the visual search task of Experiment 4, and there were no changes in geometry between the two experiments or within each recording session. This ensured that any error patterns observed in Experiment 3 would be indicative of the extent of the error for Experiment 4, and consequently that the pupil data for Experiment 4 could be corrected using the data-driven procedures outlined by Brisson et al. Based on the findings of Brisson et al. and the other studies described in Section 5.1.1, it was predicted that pupil size would vary significantly as a function of both horizontal and vertical gaze position.

\subsubsection{Method}

\section{Participants}

All participants who took part in Experiment 4 took part in Experiment $3(n=27)$, once at the beginning of each of the two experimental sessions (54 sessions in total: see Section 5.3.2 for participant information).

\section{Task, stimuli and apparatus}

The task was a close replication of Brisson et al. (2013). Participants tracked a small blue ellipse $\left(1.8^{\circ} \times 1.8^{\circ}\right)$ for $30 \mathrm{~s}$ as it traced a circular pattern on a grey background counterclockwise around the screen ( 6 full rotations). The task is a convenient method for measuring pupil size across a range of $x$ and $y$ gaze coordinates on the screen. Crucially, the motion path of the ellipse mapped the horizontal and vertical limits of the portion of the screen where stimuli could appear in the visual search task of Experiment $4\left(20^{\circ} \times 20^{\circ}\right.$, see Figure 5.1). The stimuli were presented on a 24-in. Ilyama monitor running at a resolution of $1024 \times 768$ with a refresh rate of $144 \mathrm{~Hz}$. The monitor and eye tracker were enclosed such that the only direct illumination came from the display screen. The screen was viewed from a distance of $40 \mathrm{~cm}$, which was maintained by a chin rest and forehead bar. Using a colorimeter (ColorCAL MKII, Cambridge Research Systems), the surface luminance of the grey background was recorded as $73.56 \mathrm{~cd} / \mathrm{m}^{2}$, and the surrounding dark light of the unused portion of screen as 0.54 $\mathrm{cd} / \mathrm{m}^{2}$. Pupil size and gaze data were recorded monocularly (left eye) using an EyeLink 1000 (SR Research, Ontario, Canada) system in tower mount configuration sampling at $1000 \mathrm{~Hz}$. Stimulus presentation was managed with Experiment Builder (SR Research, Mississauga, Ontario, Canada). 


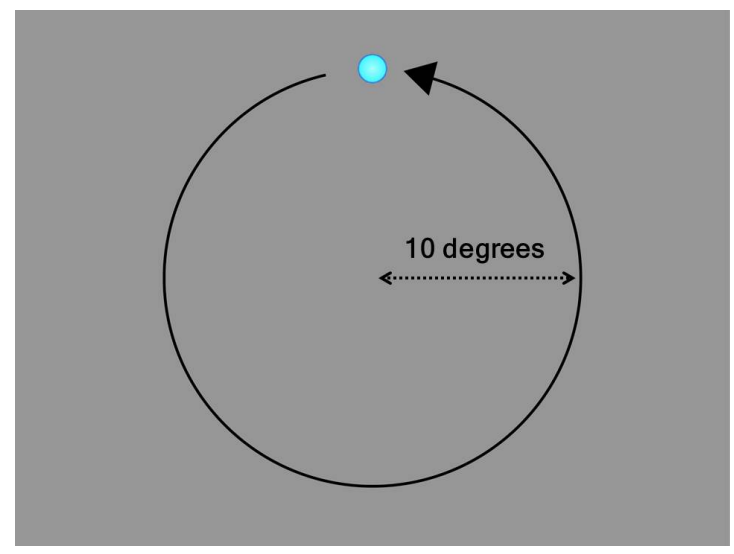

Figure 5.1. The tracking object with its trajectory shown by a circular arrow (not to scale). The path of the object mapped the horizontal and vertical limits of the portion of the screen where display items could appear in the visual search task of Experiment 4.

\section{Procedure}

Experiment 3 was completed by all participants from Experiment 4 at the start of each session. A 5-point calibration routine was first performed, then participants received on-screen instructions to fixate the ellipse in its starting position at the top of the screen and to track it as closely and accurately as possible without trying to anticipate its motion. Trials were initiated by the participant with a button press when they were ready to begin. Pupil area and gaze coordinates were recorded throughout.

\section{Data processing and statistical analysis}

Pupillometry data were preprocessed using the methods outlined in Chapter 3. Eyeblinks were first reconstructed with linear interpolation and the data were then smoothed with a third-order Butterworth filter (4 Hz cut-off). The average amount of data that were replaced by blink interpolation across all sessions was $2.46 \%$. As per Brisson et al. (2013), the relationship between pupil size and point of gaze was quantified using multiple linear regression. This was done for each individual recording session using the following formula:

$$
P^{\prime}=b_{0}+b_{1} X+b_{2} Y+e
$$

Where $P^{\prime}$ is the estimated pupil size, $X$ and $Y$ are the horizontal and vertical gaze coordinates, and $e$ is the residual error. The coefficients from the regression models for each session were then used to correct pupil size measurements as follows: 


$$
P_{c}=P-b_{1} X-b_{2} Y
$$

Where $P_{c}$ is the corrected pupil size, $P$ is the recorded pupil size, $X$ and $Y$ are the relevant gaze coordinates, and $b_{1}$ and $b_{2}$ are the coefficients from the regression model.

\subsubsection{Results}

A significant regression equation was found for all sessions, with all predictors being significant (all $p s<.05$ ). Individual regression models are not explored in detail as they simply reflect factors that were not under experimental control, such as between-session differences in geometrical layout and the average participant pupil size in native pixel units for each individual recording session. The average parameters across all regression models however can give some general insight into the pattern of the PFE. On average, $34.36 \%$ of variance (mean $R^{2}=.343$, ranging from .048 to $.734, S D=.182$ ) in pupil data was accounted for by changes in $X$ and $Y$ gaze coordinates. The average regression coefficient for $X$ was -0.483 (ranging from -1.146 to -0.122 , $S D=0.276$ ), and for $Y$ it was 0.009 (ranging from -0.712 to $0.518, S D=0.257$ ). From this it can be gleaned that pupil size was affected mostly by changes in horizontal gaze position and that rightward horizontal shifts in gaze were always associated with decreasing pupil size. This is appropriate given that pupil size was always recorded from the left eye, making the pupil position consistent between sessions with respect to the optical axis of the camera. On the other hand, changes in vertical gaze position caused the pupil to either increase or decrease in size, depending on the session. This can be linked to differences in eye level arising from session-specific adjustments to the chin rest of the tower-mount, which were practically necessary to enable eye tracking and ensure participant comfort. The findings described above are illustrated clearly in Figures 5.2 and 5.3: The former shows corrected and uncorrected pupil size data for all sessions (with regression lines) as a function of horizontal and vertical gaze position, and the latter shows grand averages of pupil size and gaze position between all sessions as a function of time. The effects of applying the correction with Equation 5.2 are visualised in the right-hand panels of Figure 5.2 and the bottom panel of Figure 5.3.

\subsubsection{Discussion}

The purpose of Experiment 3 was to measure the PFE and derive correction coefficients that could potentially be used to correct the pupil data from Experiment 4 for gaze position artefacts. Participants completed an adaptation of the ellipse tracking routine 

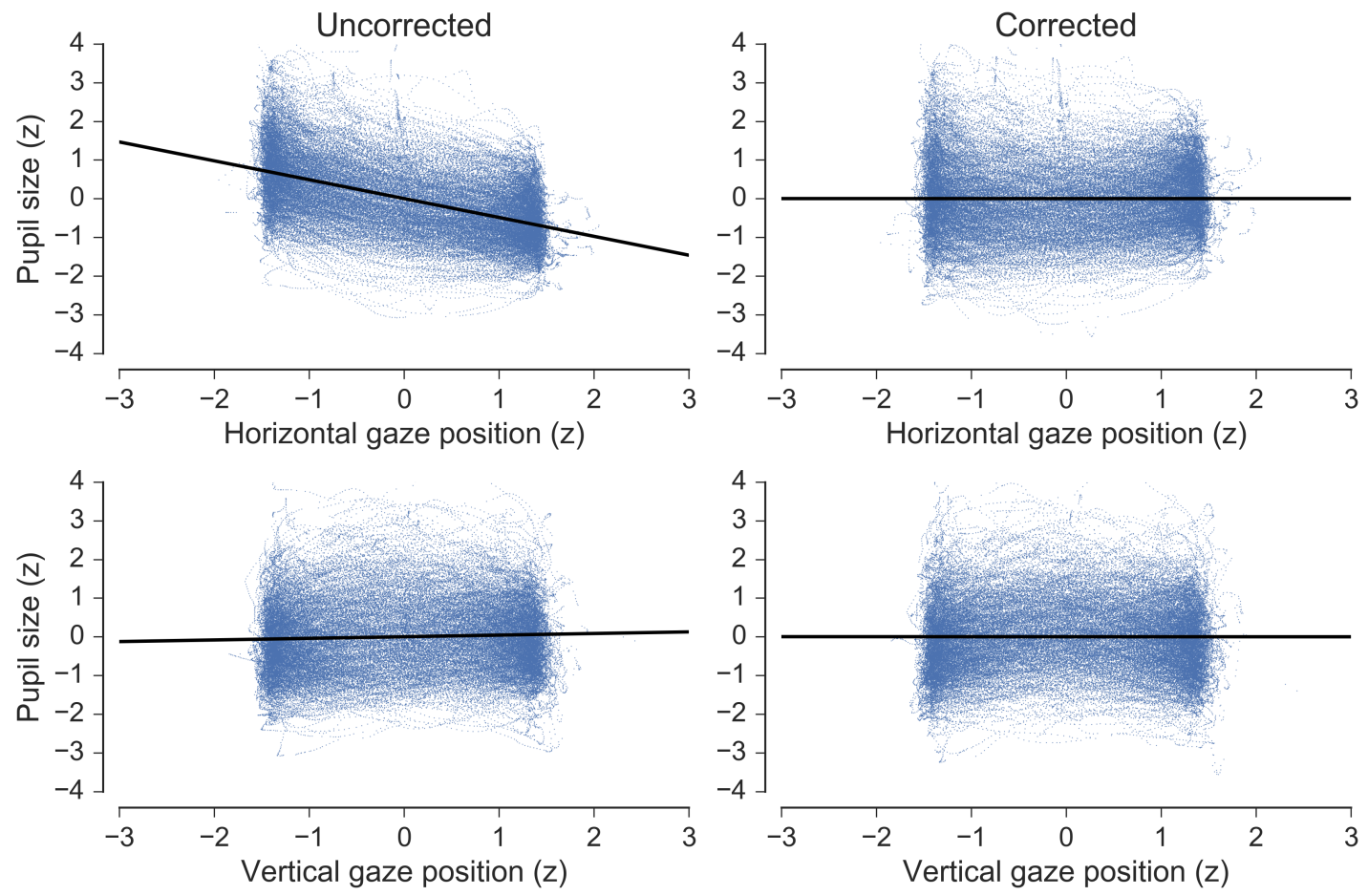

Figure 5.2. Pupil size across all sessions in Experiment 4 as function of horizontal (top row) and vertical (bottom row) gaze position. Uncorrected pupil data are shown in the left column and corrected data in the right column. 

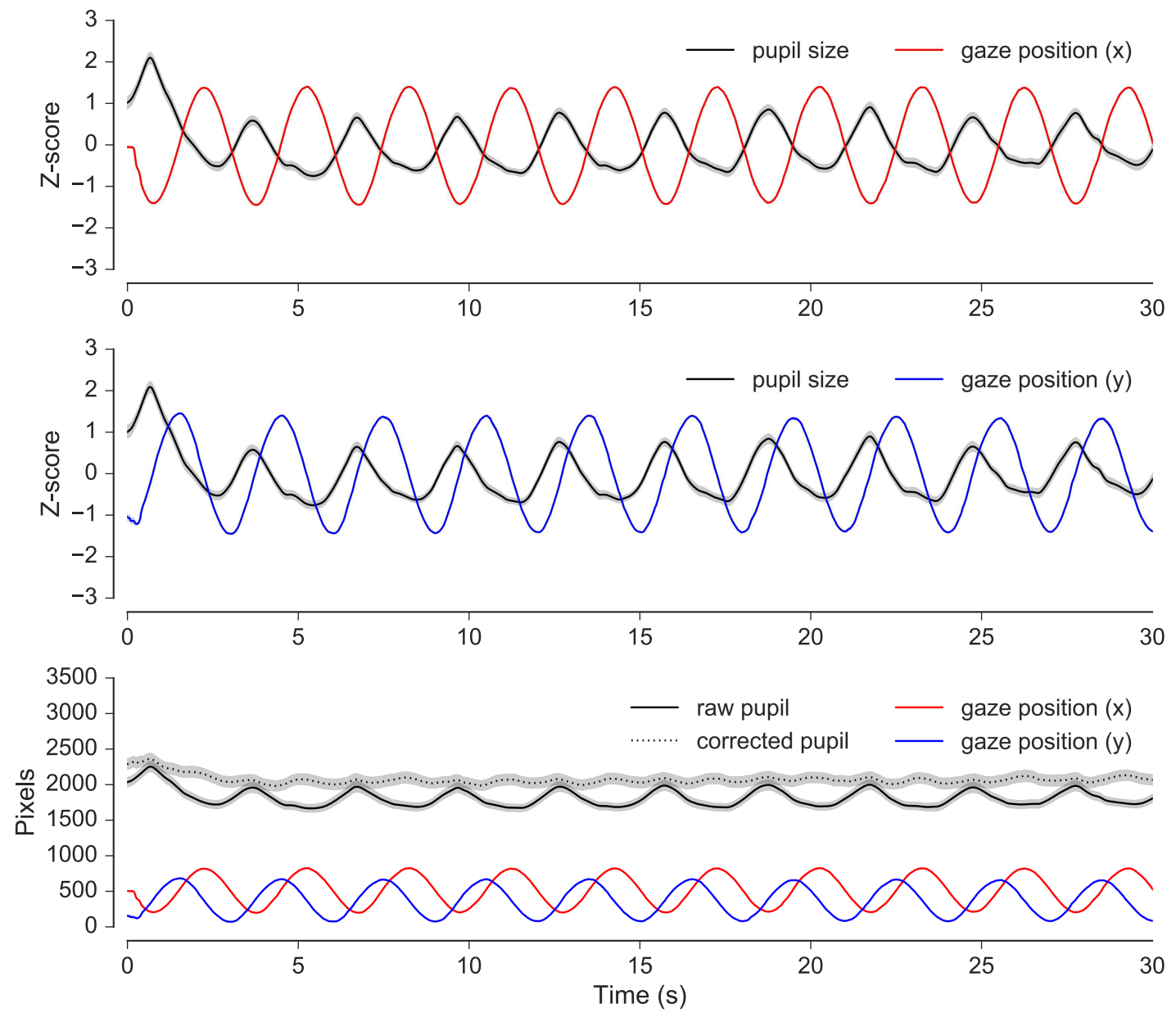

Figure 5.3. Pupil size and gaze position as a function of time in Experiment 4 (averages across sessions). The top and middle panels illustrate the covariance between pupil size and horizontal and vertical gaze position, respectively. The bottom panel shows pre and postcorrected pupil data together with the horizontal and vertical gaze coordinates in pixel units. The covariance between pupil size and gaze position is noticeably diminished after the correction was applied. Shaded areas surrounding the pupil traces reflect the SEM (bootstrapped, 5000 iterations). 
used by Brisson et al. (2013), which allows for repeated assessment of pupil size across a range of $x$ and $y$ gaze coordinates in a short period of time. As observed by Brisson et al., despite the minimal cognitive demands of the task, pupil size was found to vary systematically as a function of gaze position throughout the $30 \mathrm{~s}$ recording, showing a sinusoidal variation which closely mirrored the sine and cosine functions of the circular motion of the ellipse (Figure 5.3).

Regression analyses revealed that pupil size in every session was significantly predicted by gaze position, with all individual predictors being significant. In the current experiment, an average of $34.36 \%$ of the variance in pupil size was explained by changes in gaze position, which is considerably higher than the value of $9.9 \%$ reported by Brisson et al. (2013) for the EyeLink system. The difference here can be linked to two factors. First, the trajectory of the tracking object in the current adaptation spanned twice as many degrees of vertical visual angle and $7^{\circ}$ more horizontal visual angle, which ensured larger deviations in gaze position relative to the optical axis of the camera, and therefore larger PFE. Second, Brisson et al. measured pupil size in diameter with an EyeLink 1000 system in desktop configuration, whereas in the current experiment pupil size was measured in area with the same system but in tower-mount configuration. This means that the average recorded pupil size for this experiment $(M$ $=1817, S D=442$, ranging from 912 to 3103 , in native pixel units) was most likely larger on average than it was for Brisson et al.'s experiment, which would lead to higher covariance (Brisson et al. did not report pixel measurements so this can not be verified).

Using Equation 5.2 it was possible to correct pupil size such that its covariance with gaze position was noticeably diminished. The flattening of regression slopes in Figure 5.2 and the reduced amplitude of sinusoidal variation in corrected pupil size in the bottom panel of Figure 5.3 both illustrate this effect. Overall, these observations suggest that the regression models from the current experiment can be safely used to correct the pupil data from Experiment 4 for gaze position artefacts on a trial-by-trial basis, as the experimental layout did not change between experiments. However, one potential limitation of this data-driven approach to correcting pupil data is its implicit assumption that pupil size does not actually depend on gaze position, and that any observed relationship is consequently artefactual. As Mathôt, Fabius, Van Heusden, and Van der Stigchel (2018) point out, this assumption may be flawed. For example, if participants are required to make uncomfortable eye movements to foveate extreme parts of the screen, the pupil may dilate because of the mental and physical effort involved with this action. Observing such effects in a saccade and fixate task, Mathôt, Melmi, and Castet (2015) opted against data-driven correction, as in this case it would 
have corrected true cognitive effects as though they were artefactual. Another issue specific to the current design is that participants began trials with a button-press, the pupillometric effects of which are noticeable at the beginning of trials. As this may have compromised the quality of the regression analyses, a more optimal solution in a future implementation would be to omit the button press, or have the motion pattern begin following a variable delay after a button press.

\subsection{Experiment 4}

\subsubsection{Introduction}

Experiment 4 was designed with the aim of examining the pupillometric effects of effort and target detection, as reported by Porter et al. (2007) and Klingner (2010a), in further detail. First, Porter et al. found greater pupil responses in search for 1 vs. 2 targets and interpreted this in terms of a memory component associated with the discovery of the first target. If this effect was indeed linked to memory, it should scale with number of targets in the search, because the demands on locational memory would increase with each successive target discovery. Second, all trials in Porter et al.'s experiments involved a binary decision and a button response which appeared to evoke a large pupil dilation, making it hard to evaluate the relative contribution of this task component to the observed pupil responses. It would be interesting to see how the pupil dynamics were changed towards the end of search if decisions and button responses were not always required. Finally, Klingner (2010a) reported effects of target detection with fixation-aligned pupil responses, but the averages were taken across a small sample of subjects and the statistical significance of the effect was not quantified. The reliability of these findings and whether they can be observed between subjects is therefore a question that remains to be addressed.

With the above directions in mind, a visual search task was devised combining aspects of the designs used by Porter et al. (2007) and Klingner (2010a). Participants searched for forward facing Landolt ' $C$ 's in arrays of heterogeneously oriented distractor 'C's. Trials contained up to three targets, and the participants had to report the number of targets via mouse-click at the end of the trial. A maximum search time of $10 \mathrm{~s}$ was allowed, but participants were also given the option to terminate the search with a button press at any time if they thought they had found all of the targets. If pupil dilation is linked to spatial memory load it should be lowest for trials without targets and should increase as the number of targets increases, as the location of each additional target must be remembered to ensure accuracy and facilitate efficiency. Termination searches 
should exhibit large peaks at the time of search offset due to the effects of the decision process and motor response, whereas nontermination-searches should not exhibit these effects. Finally, fixations on targets should evoke transient pupil dilations compared to fixations on distractors.

\subsubsection{Method}

\section{Participants}

Twenty-seven participants ( 19 women; age range $18-31$ years, $M=21.74, S D=3.51$ ) completed the experiment voluntarily or in exchange for course credit. All participants were students at Swansea University reporting normal or corrected-to-normal acuity and colour vision. Participation was voluntary or in exchange for course credit. The experimental protocol was approved by the Ministry of Defence Research Ethics Committee and the Department of Psychology Ethics Committee at Swansea University. Written informed consent was obtained from each participant.

\section{Design}

A repeated-measures design was used, with the number of targets present in the search array as the only systematic experimental manipulation (Targets: $0,1,2,3$ ), as per Klingner (2010a). Participants searched for forward-facing Landolt 'C's in arrays of heterogeneously oriented distractor ' $\mathrm{C}$ 's, rotated through $90^{\circ}, 180^{\circ}$ or $270^{\circ}$, as in the 'hard search' conditions in the experiments of Porter et al. (2007). Set size was fixed at 30 display items for all trials. Arrays were displayed for $10 \mathrm{~s}$ or until participants opted to terminate the search by pressing the space bar, on belief of finding all targets. Participants indicated the number of targets thought to be present via mouse-click after the search. The experiment consisted of 144 experimental trials spread evenly across two sessions, both of which lasted approximately $1 \mathrm{hr}$ and were completed within a week of one-another. In each session, participants completed 4 practice trials (one for each level of Targets) before undertaking 4 blocks of 18 experimental trials. The same set of 152 search arrays was used for each participant in the experiment (37 for each level of Targets), all of which were prepared in advance along with a luminance mask to match (see below). An example search array for a trial with 3-targets is shown together with its mask in Figure 5.4. The order of array presentation was randomised across the whole experiment. Accuracy and search time (from search onset until termination or the maximum time of $10 \mathrm{~s}$ ) were recorded for each trial, as well as continuous recordings of gaze position and pupil size. 


\section{Stimuli and apparatus}

Stimuli were arrays of Landolt 'C's enclosed within an unbroken ring, as used previously by Porter et al. (2007). They were created using vector graphics software and custom python scripts. Individual target and distractor image tiles were made in Adobe Illustrator by placing a black circle with a 2.7-point stroke width and 30 px diameter on a grey background. Within this circle was placed another of half the diameter, and a grey rectangle was then used to punch out approximately $20 \%$ of the circumference on the right hand side of the inner circle to give the ' $\mathrm{C}$ ' shape. This formation was then exported as a $60 \times 60 \mathrm{px}$ image file in JPEG format. An image file was also created for each of the distractor orientations, the fixation circle and the masking element (identical to the targets and distractors but for an unbroken inner circle). The Python Image Library was then used to make each of the 152 stimulus arrangements. The fixation circle was placed at the centre of a $1024 \times 768$ px canvas and the display elements were randomly allocated to predefined locations on a $10 \times 10$ virtual grid $(600 \times 600$ pixels) with a uniform grey background. The four locations at the centre of the grid were excluded to avoid rapid processing of display items appearing close to fixation at the beginning of a trial. Spatial clustering of targets was avoided by randomly allocating each target at first to a quadrant of the grid, and then to a location within that quadrant. A mask was made to match each search array (see Figure 5.4).

Stimuli were presented on a 24-in. Ilyama monitor running at a resolution of $1024 \times 768$ with a refresh rate of $144 \mathrm{~Hz}$. The monitor and eye tracker were enclosed such that the only direct illumination came from the display screen. The screen was viewed from a distance of $40 \mathrm{~cm}$, which was maintained by a chin rest and forehead bar. From this distance, targets and distractors subtended approximately $1^{\circ} \times 1^{\circ}$ of visual angle and the portion of the screen where targets could appear subtended $20^{\circ} \times 20^{\circ}$. Using a colorimeter (ColorCAL MKII, Cambridge Research Systems), the surface luminance of the grey background was recorded as $73.56 \mathrm{~cd} / \mathrm{m}^{2}$, and the surrounding dark light of the unused portion of screen as $0.54 \mathrm{~cd} / \mathrm{m}^{2}$. Pupil size and gaze data were recorded monocularly (left eye) using an EyeLink 1000 (SR Research, Ontario, Canada) system in tower mount configuration sampling at $1000 \mathrm{~Hz}$. Eye level and camera position remained constant throughout the recording session for each participant. Stimulus presentation was managed with Experiment Builder (SR Research, Mississauga, Ontario, Canada). 

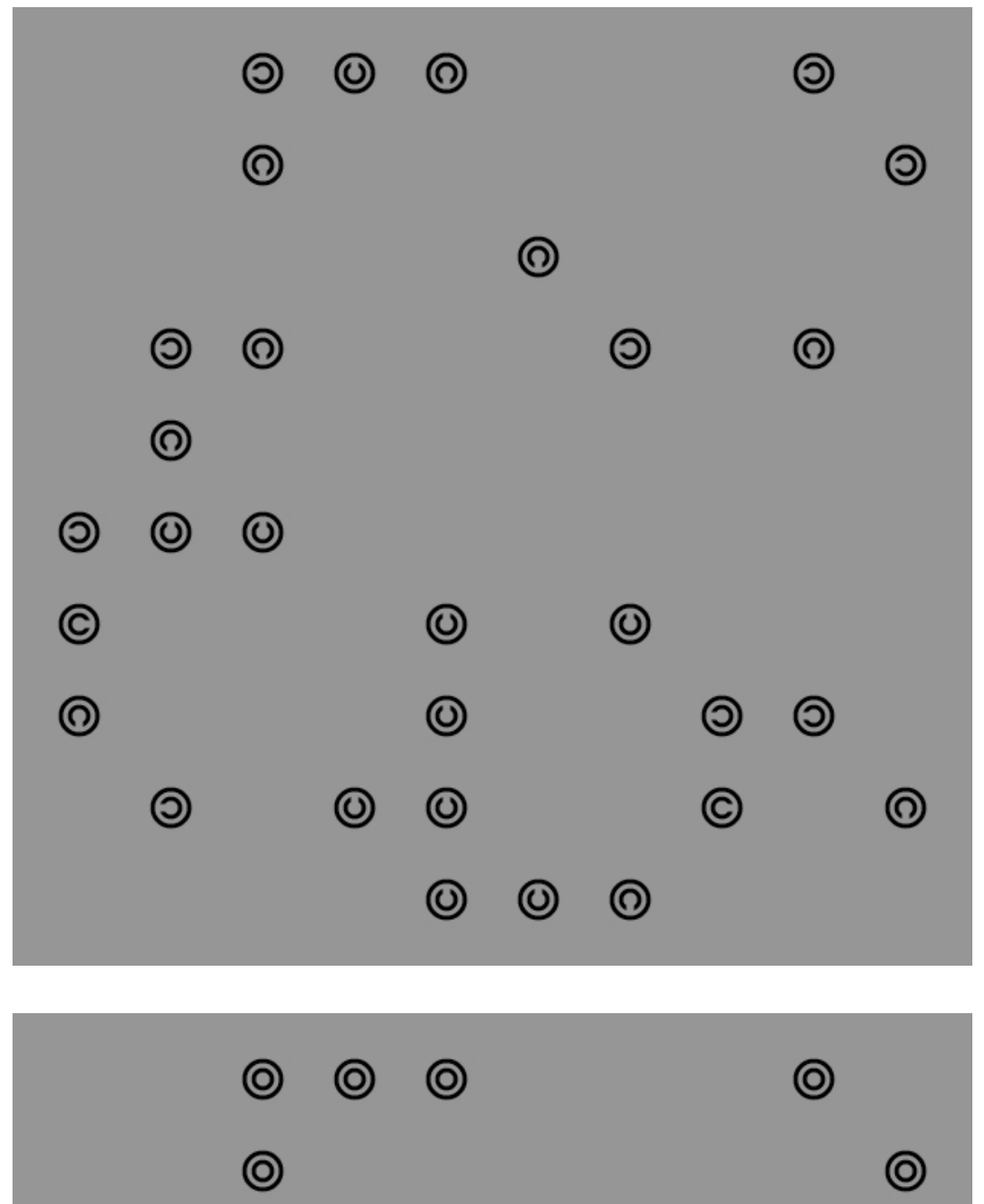

()
() (
(2)
()

(อ)

() () ()

()

()

()

() (ㅇ

()

() (

()

()

() () ()

Figure 5.4. Example 2-target search array (top) and its mask (bottom), not shown to scale. 


\section{Procedure}

Participants were instructed to find all of the targets and report via mouse-click after each trial, as accurately as possible, how many targets were present. They were informed that they had up to $10 \mathrm{~s}$ for each search, but also that they could terminate the search prematurely by pressing the space bar if they thought that they had found all of the targets. A 13-point calibration and validation routine for the eye tracker was performed at the beginning of the experiment, and then again as required throughout. Four practice trials were completed (one for each level of Targets) prior to the main experimental trials. Each trial began with a drift check followed by a $2000 \mathrm{~ms}$ fixation period. The mask array then appeared as participants maintained fixation, which served to minimise any luminance artefacts that would be associated with the appearance of the stimulus array. After $4000 \mathrm{~ms}$ the mask was replaced by the stimulus array and the search commenced. The stimulus array remained in place for $10 \mathrm{~s}$ or until participants pressed the space bar, after which the mask returned to the screen for $2000 \mathrm{~ms}$. The response screen subsequently appeared, and participants responded to the question 'How many targets were present?' by using the computer mouse to select the appropriate on-screen selection box. After the response was collected, a blank display was presented for 2000 $\mathrm{ms}$, then the trial cycle began anew. This sequence is illustrated in Figure 5.5, and Figure 5.6 shows gaze and fixation data for a typical trial visualised over the stimulus array.

\section{Data processing}

Pupillometry data were preprocessed using the methods outlined in Chapter 3. Eyeblinks were first reconstructed with linear interpolation. The pupil data were then corrected for gaze position using Equations 5.1 and 5.2, with the coefficients from the session-specific regression models. Data were then smoothed with a third-order Butterworth filter (4 Hz cut-off). The average amount of data replaced by blink interpolation across all participants that were included in the analysis was $5.15 \%$. Whole-trial analysis and fixation-aligned pupillary response averaging were used to explore pupil data, the details of which are given separately below. The behavioural performance measures were accuracy, search time and the proportion of termination-searches. For search time, the analysis was restricted to correct-only trials. 


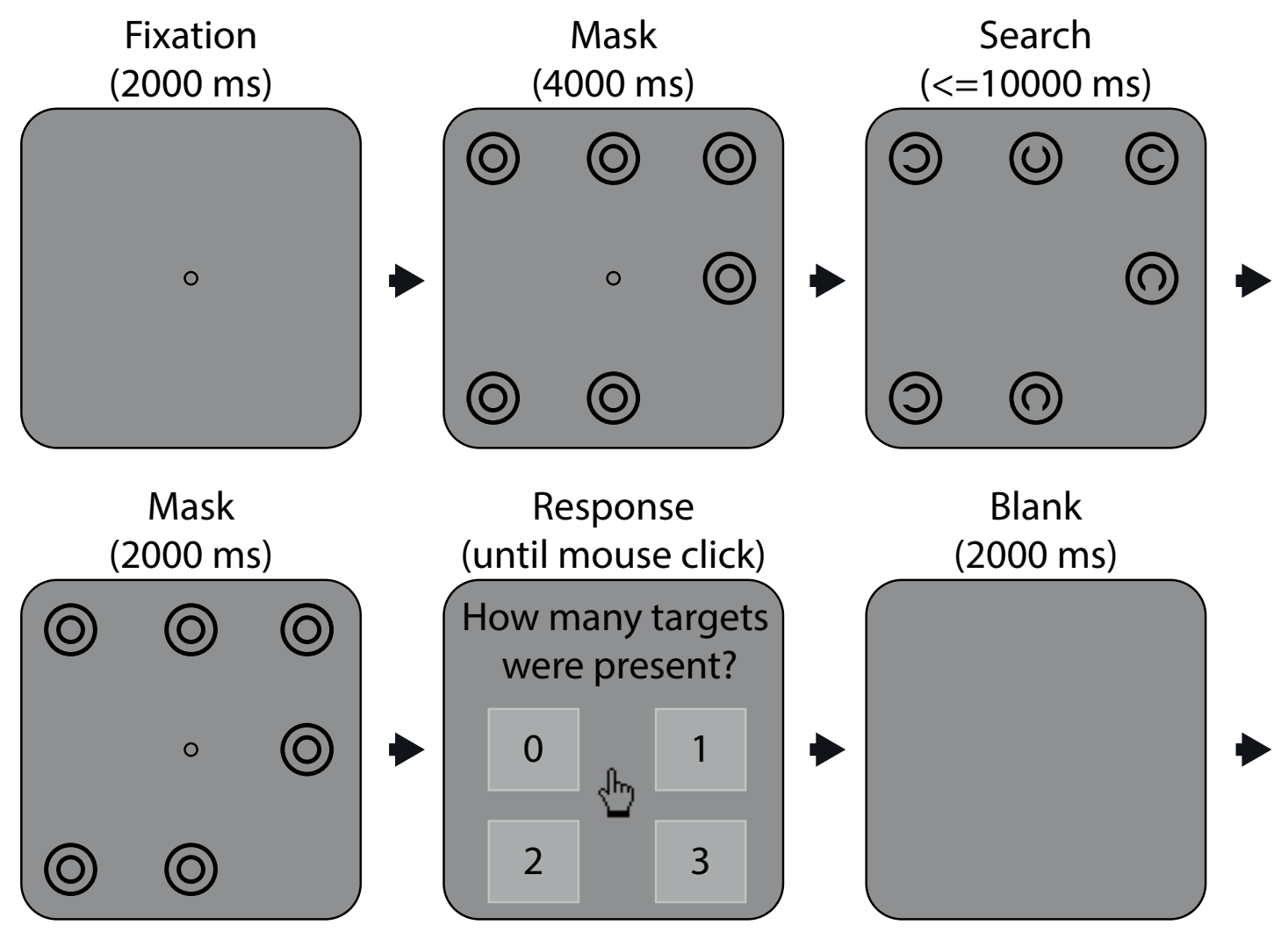

Figure 5.5. The trial sequence used in Experiment 4. Each trial began with a 2000 ms fixation. Then the mask appeared, with the fixation circle remaining on the screen. After 4000 ms the mask transitioned to the search array, which remained on screen for either $10 \mathrm{~s}$ or until participants indicated they had finished the search by pressing the space bar. The mask then reappeared for $2000 \mathrm{~ms}$ prior to the response screen, where participants responded to the question 'How many targets were present?'. The trial cycle ended with a 2000 ms blank screen, followed by a drift check. Displays have been simplified for purposes of illustration. 


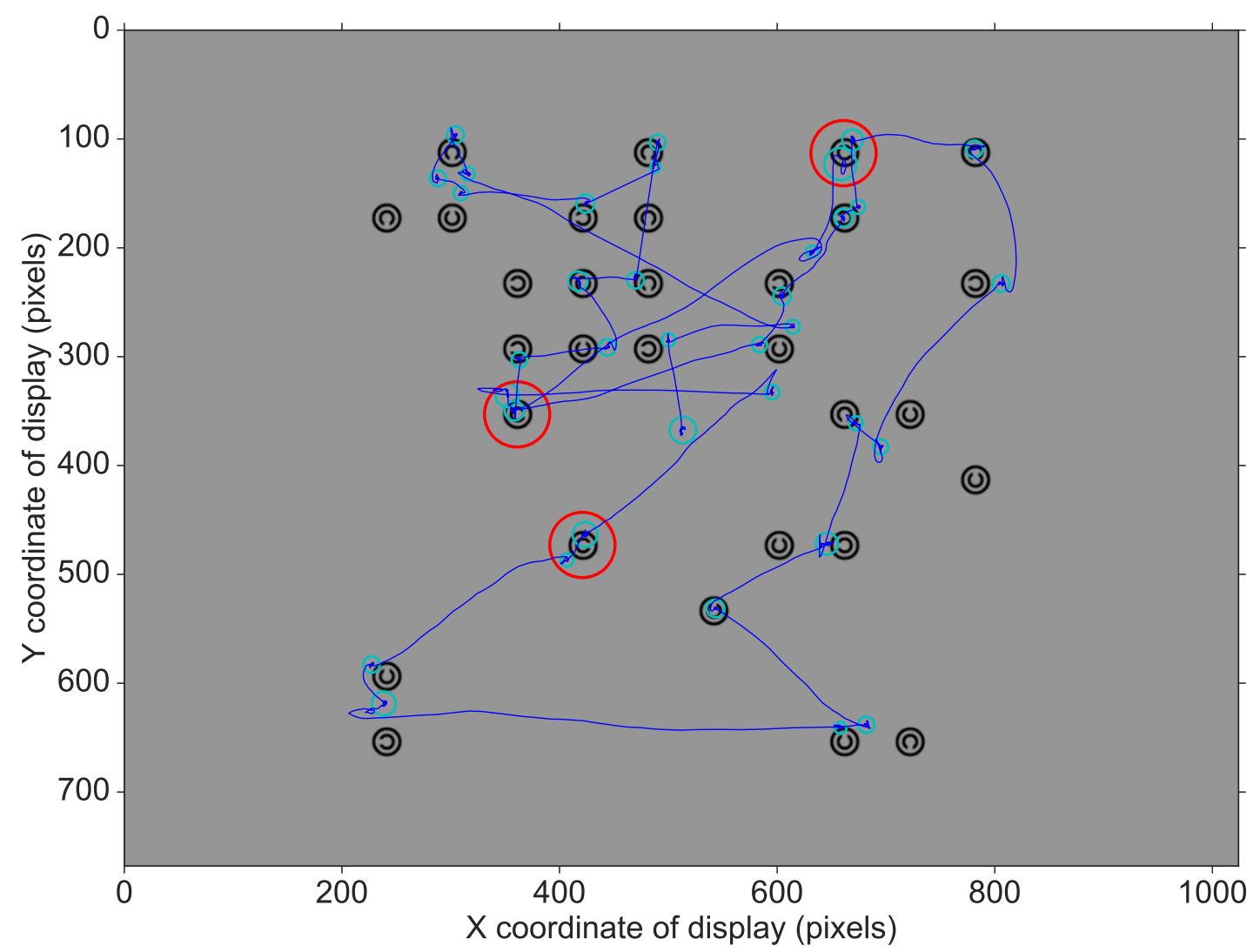

Figure 5.6. Example trial data for a 3-target trial with image and interest area overlay. Gaze data are shown in dark blue and fixation data in light blue, with the size of the circles representing fixation duration. The red lines delimit the extent of the interest areas that were used to classify fixations as belonging to a particular item (shown here only for targets). 


\section{Whole-trial analysis of pupil data}

Whole-trial pupil analysis was conducted to assess the effect of Targets on the general pattern of pupil responses. Average pupil traces for each level of Targets were timelocked to search onset and offset. The data between these anchor points were then normalised to account for differences in search time. This method ensures that pupil traces are compared at roughly equivalent points of task-related processing (Porter et al., 2007). Pupil data were expressed as \%-modulation from baseline, with the baseline calculated as mean pupil size across $1000 \mathrm{~ms}$ prior to search onset. Only trials which resulted in a correct response were included in the analysis.

\section{Fixation-aligned pupillary response averaging}

Fixation-aligned pupillary response averaging was used to gain insight into pupil dynamics surrounding target and distractor fixation during search. This was achieved following the general method outlined by Klingner (2010a), but with some differences to how fixations were identified. Klingner identified fixations with a dispersion threshold technique described by Widdel (1984), which resulted in fixations having a minimum duration of $160 \mathrm{~ms}$. In the current experiment, fixations were identified using EyeLink's standard event parsing algorithm. Accordingly, any sample that did not exceed the motion $\left(0.1^{\circ}\right)$, velocity $\left(30 \%\right.$ s) or acceleration $\left(8000^{\circ} / \mathrm{s}^{2}\right)$ thresholds for saccade detection was deemed to be part of a fixation.

After data collection, the following procedure was used to identify target and distractor fixations for further analysis. Brief fixations $(<100 \mathrm{~ms})$ were merged with neighbouring fixations $\left(<1^{\circ}\right.$ visual angle), or discarded. All remaining fixations were then assigned to display items if they fell within $1.25^{\circ}$ of their centre, and those which could not be assigned to a display item in this way, or were consecutive fixations on the same display item, were discarded. To ensure a level of parity in the duration of target and distractor fixations, all fixations whose duration was below $120 \mathrm{~ms}$ or above 3 standard deviations from the mean of all target fixations were discarded. Finally, to ensure as far as possible that fixation-aligned responses would only reflect cognitive components associated with target and distractor fixation, it was necessary to remove those which may have been contaminated by display changes or button responses. Fixations which occurred within the first $1000 \mathrm{~ms}$ of search and those which occurred within $2500 \mathrm{~ms}$ of a button response were therefore not included in the analysis. The former of these criteria (similar to Klingner's removal of the first 5 fixations from each trial) should ensure that pupil responses would not be systematically confounded by the small luminance changes associated with the transition between the mask and search 
arrays. The latter ensured that responses would not be systematically confounded by the effects of button responses, which begin to emerge up to $1500 \mathrm{~ms}$ prior to the recording of the response (e.g. Einhäuser et al., 2010; Richer \& Beatty, 1985). The frequency distributions of all remaining target and distractor fixation durations are shown in Figure 5.7. Once fixations were identified, epochs of pupil data from 500 ms prior to fixation onset until $2000 \mathrm{~ms}$ after onset were extracted. These data were expressed as \%-modulation from a baseline calculated as the mean pupil size in the 500 $\mathrm{ms}$ period prior to fixation onset.
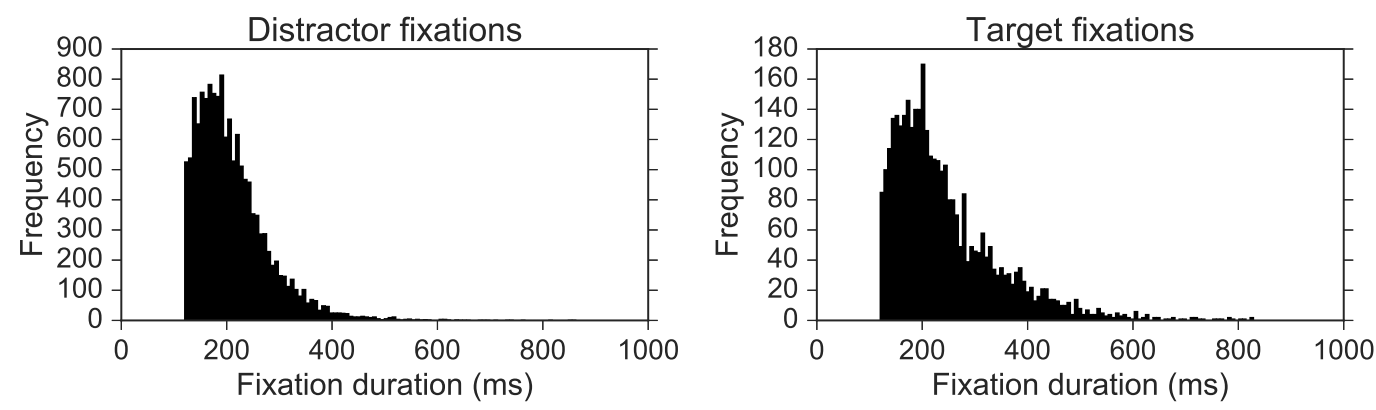

Figure 5.7. Frequency distributions for target and distractor fixation durations (bin width $=10 \mathrm{~ms}$ ).

One of the aims of the fixation-aligned response analysis was to replicate Klingner's (2010) effect of target detection, whereby on-target fixations evoked transient pupil dilation and off-target fixations did not. For this comparison, the fixation-aligned responses to targets and distractors were averaged together for each participant, and then grand-averages were taken to examine the effect between subjects, something which was not examined in Klingner's report. The next question of interest was if, and how, pupil responses differed between initial fixations and refixations on an item. Klingner reported that pupil dilation for target revisits started prior to the actual fixation, but did not check for an effect of distractor revisitation. In fact, Klingner compared target fixations to 'control fixations', the principal criteria for which was that they were at least $5^{\circ}$ from a target item. This means that his control fixations may have been fixations on distractors, but could just as easily have been fixations on blank portions of the screen. Therefore it is unclear whether refixations on distractors would cause the pupil to dilate. The final question of interest was whether fixation-aligned pupil responses were sensitive to the accumulation of memory load that would be expected as more targets are discovered during search (e.g. Porter et al., 2007). To examine this, target fixations from trials with multiple targets (i.e. Targets: 2, 3) were grouped by the order in which they were discovered. After virtually all third target discoveries, 
a keypress was present, meaning that these responses could not serve as a reliable basis for inferences about cognitive processes. The analysis was therefore restricted to first and second target discoveries, which represent an accumulation of load without contamination from cognitive and motor processes triggered by the end of search.

\section{Statistical analysis}

Performance measures were analysed using one-factor (Targets: 0, 1, 2, 3) repeatedmeasures ANOVAs. Where appropriate, $p$-values were adjusted for violations of sphericity using the Greenhouse-Geisser correction. Scalar values of pupil data (e.g. whole-trial averages) were also analysed in this way. The statistical evaluation of pupil modulation from baseline and differences in modulation between conditions was achieved using two-tailed non parametric permutation tests with cluster-based correction for the multiple comparisons problem (Maris \& Oostenveld, 2007). F-tests were used for exploring the main effects of variables with more than two conditions (i.e. Targets) and $t$-tests for comparisons between two conditions (e.g. target vs distractor). The significance thresholds for test statistics were determined theoretically from the appropriate degrees of freedom, with $\alpha=0.05$.

\section{Trial and participant exclusions}

Individual trials were excluded from statistical analysis if over $50 \%$ of the pupil data were interpolated. One participant was excluded from all analysis for terminating $100 \%$ of trials and attaining only chance accuracy (the same participant who did not yield usable correction data from Experiment 3). Exclusion of this participant did not affect the overall pattern of results. All remaining participants attained at least $78 \%$ accuracy across all conditions.

\subsubsection{Results}

\section{Performance data}

The results for the performance measures are summarised in Figure 5.8. All participants included in the analysis attained at least $78 \%$ accuracy across all trial types. There was a significant main effect of Targets on accuracy, $F(2.29,57.33)=68.08, p<.001$, $\eta_{p}^{2}=0.73$, which was characterised by a downward trend from $98.8 \%(S D=2.9 \%)$ in 0 -target trials to $77.5 \%(S D=9 \%)$ in 3-target trials. Bonferroni-corrected follow-up tests revealed all pairwise comparisons to be significant (all $p \mathrm{~s}<.001$ ), except for $1 \mathrm{vs}$ 
2 targets $(p=.127)$. These results are generally consistent with those from previous experiments involving search for multiple targets, where increasing numbers of targets lead to more subsequent search misses (Adamo et al., 2013; Biggs, 2017; Cain et al., 2013; Gorbunova, 2017).

A significant main effect of Targets was observed for both search time, $F(1.67$, $41.75)=55.57, p<.001, \eta_{p}^{2}=0.69$ (middle panel of Figure 5.8), and the proportion of self-terminated trials, $F(1.76,44.19)=17.79, p<.001, \eta_{p}^{2}=0.42$ (right panel of Figure 5.8). These effects were inversely related to one another, with search time being shorter in conditions where more self-terminations were observed. There were no significant differences in either of the measures for all possible comparisons involving trials with 0-2 targets (all $p \mathrm{~s}>.05$ ), but 3-target trials differed significantly to all other trial types for both measures (all $p s<.001$, Bonferroni-corrected). These findings are consistent with the demands of the task in that participants knew they could terminate search without compromising accuracy after the discovery of a third target.
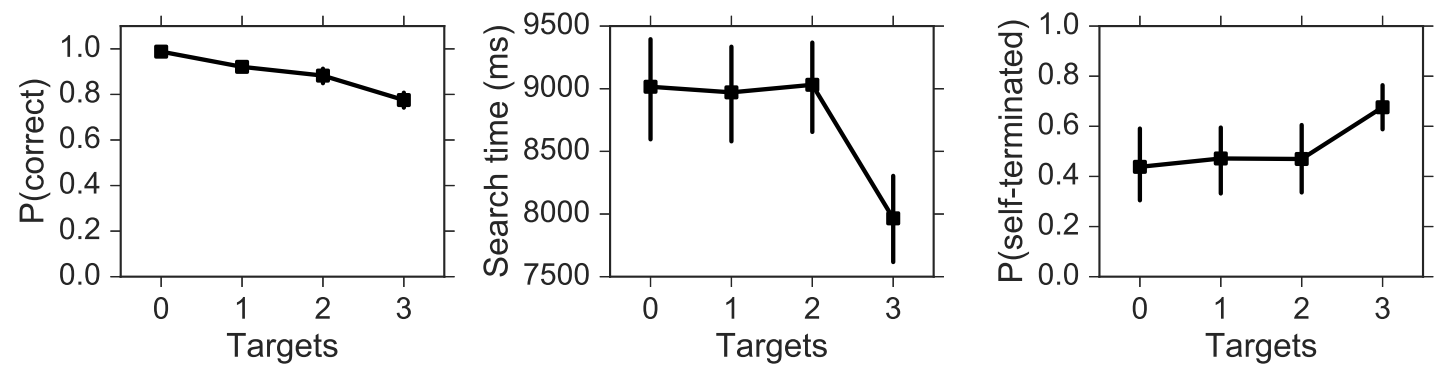

Figure 5.8. Accuracy (left), search time (middle) and the proportion of self-terminated searches for each level of Targets. Error bars, where visible, show 95\% confidence intervals (bootstrapped, 1000 iterations).

\section{Pupil data}

Whole-trial analysis of pupil data was first conducted to gain insight into the general pattern of pupil responses across the task. Figure 5.9 shows the grand average pupil traces for each level of Targets, time-locked to search onset, phase of performance and search offset. Pupil size increased prior to onset ${ }^{4}$, and then continued to increase once the search began, peaking at around $2000 \mathrm{~ms}$ into the search. After this initial peak, pupil size began to decrease slightly, but then increased again towards the end

\footnotetext{
${ }^{4}$ The rising baselines can be attributed to the luminance decrement associated with the appearance of the stimulus mask. Also, the fixed $4000 \mathrm{~ms}$ duration of the mask display may have afforded accurate anticipation of the appearance of the stimuli, something which is known to affect pupil size (Kahneman $\&$ Beatty, 1966, 1967). A simple jitter routine might have ameliorated this effect.
} 
of the search. All conditions followed this general pattern, although there was a more pronounced dilation and subsequent peak at search offset for 3-target trials compared to all other trial types. This was at once recognised as an effect of self-termination, which was more frequent in trials with three targets (right panel of Figure 5.8). To confirm that this was indeed the source of the effect, pupil traces at search offset were grouped by whether the trial was self-terminated or not. On trials which were self-terminated, the pupil began to dilate at least $1000 \mathrm{~ms}$ prior to search offset and peaked shortly afterwards. This pattern differed significantly from trials that were not self-terminated, where pupil size remained relatively constant around search offset $(p<.05$, clustercorrected permutation test: see Figure 5.11). This finding can readily be attributed to the effects of the decision to terminate the search and the subsequent motor act, both of which have been observed in similar contexts (e.g. Einhäuser et al., 2010; Richer \& Beatty, 1985). A one-factor ANOVA conducted on the average pupil responses revealed no significant main effect of Targets on pupil size $(p>.05)$.
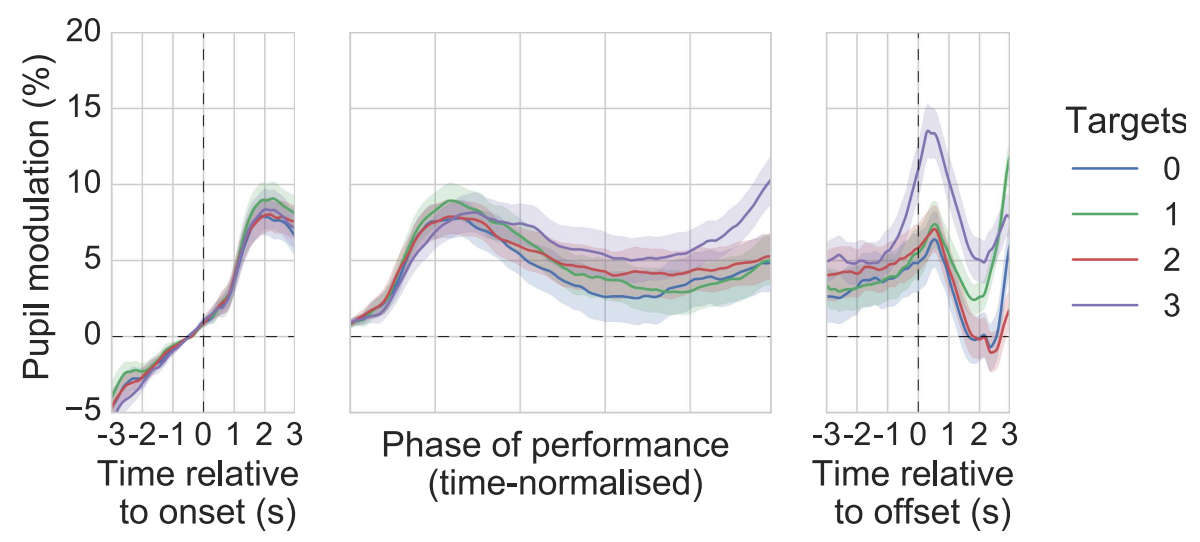

Figure 5.9. Average pupil traces for each level of Targets. The data in the left and right panels are time-locked to stimulus onset and search offset, respectively. The central panel shows pupil traces across the phase of performance (time-normalised). Shaded areas surrounding each pupil trace reflect the SEM (bootstrapped, 5000 iterations).

Although pupil data were corrected for gaze position using the regression model from the ellipse tracking routine specific to each session, they may have been affected by error that was not captured by the model and which may have been rendered systematic by regularities in participant viewing behaviour. To check whether participants consistently viewed the search arrays in a particular way, the average horizontal and vertical gaze coordinates across the phase of performance were calculated. If viewing behaviour was mostly random, then horizontal and vertical gaze coordinates would not deviate significantly from the centre of the screen. Figure 5.10 clearly shows this not to 
be the case. From the pattern of vertical gaze position it can be inferred that participants generally favoured a top-to-bottom search strategy. This means that any variability in pupil size arising from changes in vertical gaze position that was not accounted for by the correction procedure may have systematically affected the observed pupil responses.

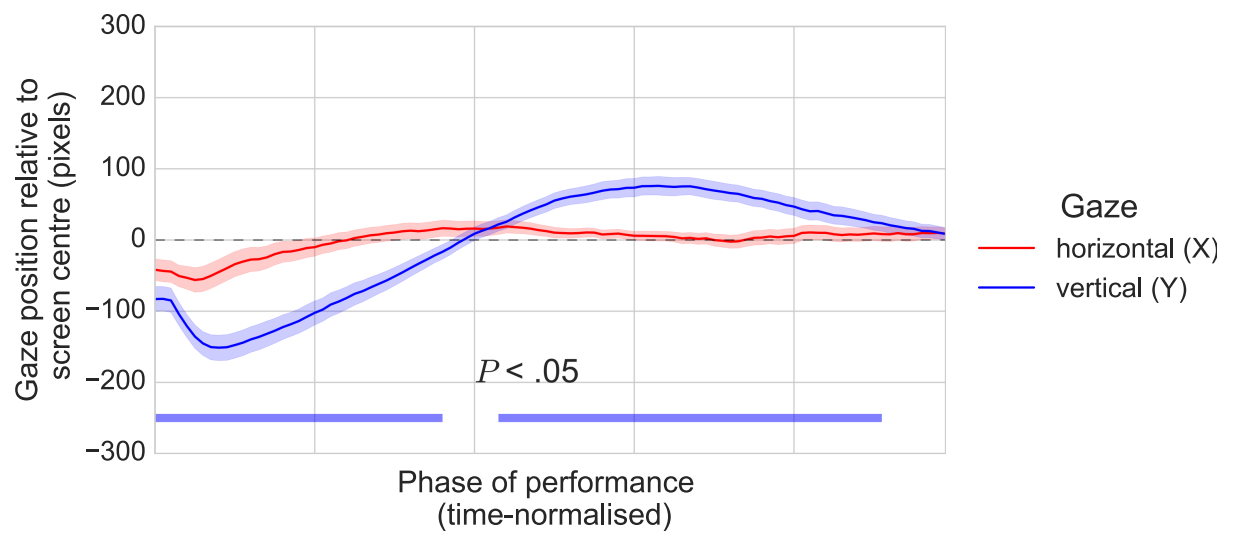

Figure 5.10. Average horizontal and vertical gaze position across the phase of performance (time-normalised). The pattern of vertical gaze position suggests that participants generally favoured a top-to-bottom strategy for viewing the stimuli. Shaded areas surrounding each pupil trace reflect the SEM (bootstrapped, 5000 iterations).

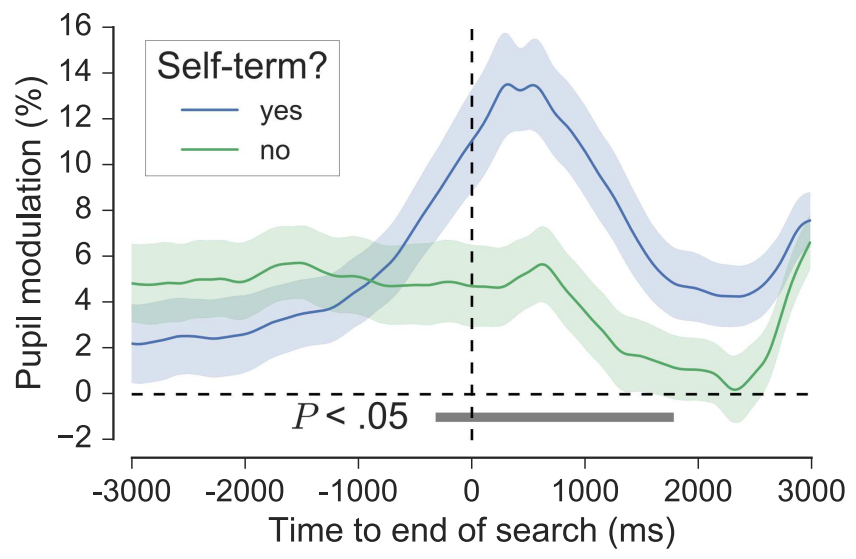

Figure 5.11. Average pupil traces for all participants at search offset, grouped by whether the trial ended with a button press or not. The horizontal grey bar indicates significant differences between the two traces, as revealed by nonparametric permutation tests (1024 permutations, $p<.05$, cluster-corrected for multiple comparisons). Shaded areas surrounding each pupil trace reflect the SEM (bootstrapped, 5000 iterations).

To gain a greater level of insight into the cognitive pupil dynamics during search, fixation-aligned pupillary response averages were calculated for all target and distractor 
fixations which fit the criteria set out in Section 5.3.2. The across-trial effect of target fixation reported by Klingner (2010a) was here replicated at the group-level, between participants (Figure 5.12). Target fixations lead to significant pupil modulation which on average reached peak of $2.02 \%$ at a latency of $680 \mathrm{~ms}$ from fixation onset, returning to baseline levels after around $2000 \mathrm{~ms}$. This effect differed significantly from the effect of distractor fixation, which was characterised by relatively stable pupil responses and no significant change from baseline $(p<.05$, cluster-corrected permutation test: see Figure 5.12). The effect of target fixation was present even in data that were not corrected for pupil foreshortening (right panel of Figure 5.12). To gain a measure of the reliability of this effect, it was visualised separately for each individual participant (Figure 5.13). Twenty-two of the 26 participants included in the analysis showed pupil responses that were greater on average for target fixations compared to distractor fixations, and at least 11 of these displayed the characteristic peak that was present in the grand-average waveform for targets. Finally, single-trial reliability was examined by selecting one participant who displayed prototypical pupil responses to targets and distractors (Participant 17) and visualising one-hundred randomly selected target and distractor epochs (Figure 5.14). This perspective shows the considerable variability in the individual epochs that contribute to the participant averages, much of which is likely to have arisen from gaze position artefacts unaccounted for by the PFE-correction procedure.

After comparing the effect of target and distractor fixation, fixations were split into discoveries (initial fixations) and revisits (refixations). Klingner (2010a) observed pupil dilation almost $500 \mathrm{~ms}$ prior to fixation onset for target revisits. This effect is not replicated here, but the responses for target discoveries and target revisits were significantly different ( $p<.05$, cluster-corrected permutation test: shown on the left panel of Figure 5.15). On average, pupil responses to target revisits reached peak modulation of $3.41 \%$ at a latency of $520 \mathrm{~ms}$ from fixation onset, whereas target discoveries reached peak modulation of $2.01 \%$ at a latency of $840 \mathrm{~ms}$. Interestingly, distractor revisits also caused significant modulation, which on average reached peak of $1.47 \%$ at a latency of $500 \mathrm{~ms}$ from fixation onset $(p<.05$, cluster-corrected permutation test: shown on the right panel of Figure 5.15).

Finally, to see if fixation-aligned responses were sensitive to the accumulation of memory load that would be expected as more targets are discovered during search, target fixations from trials with multiple targets were grouped by the order in which they were discovered (Discovery Order: 1st, 2nd). Only 1st and 2nd target discoveries were analysed, because 3rd target discoveries were virtually always followed by a 

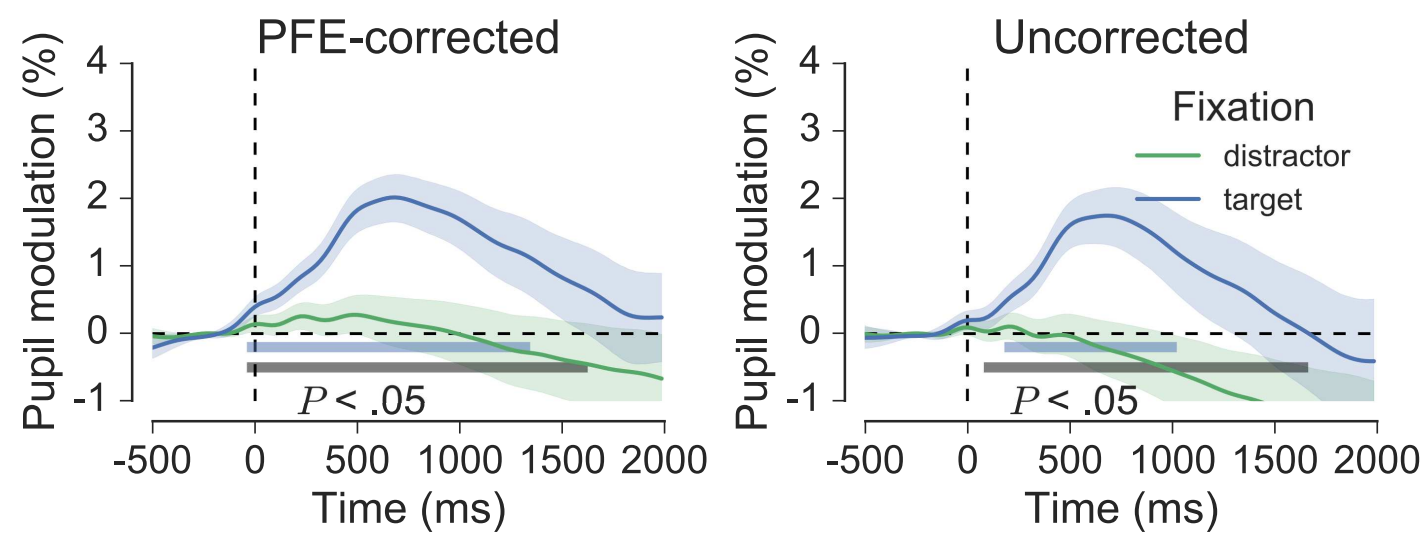

Figure 5.12. Grand-average fixation-aligned pupillary responses for target and distractor fixations, shown for PFE-corrected (left) and uncorrected pupil data (right). The horizontal blue bars denote significant modulation from baseline, and the grey bars indicate significant differences between the coloured traces, as revealed by nonparametric permutation tests $(1024$ permutations, $p<.05$, cluster-corrected for multiple comparisons). Shaded areas surrounding each trace reflect the SEM (bootstrapped, 5000 iterations). It is noteworthy that the significance of target vs. distractor fixation effect was not dependant on the data-driven PFE correction procedure.

button response shortly afterwards, and could therefore not serve as a reliable basis of inference for memory processes. The effect of Discovery Order is shown in Figure 5.16. The pupil responses associated with 1 st and 2 nd discoveries differed most consistently between 0 and $500 \mathrm{~ms}$ after fixation, but the $p$-value associated with this difference was not significant $(p>.05)$.

\subsubsection{Discussion}

The aim of this experiment was to establish a suitable method for exploring the cognitive pupil dynamics of free-viewing visual search. A previous experiment by Porter et al. (2007) suggested that pupil dilation in visual search reflects cognitive effort, possibly arising from memory load associated with remembering target locations, but the wholetrial approach to the analysis of pupillometry data did not account for the effects of other task components that were present in their design and which are known to cause pupil dilation, such as target detection (e.g. Klingner, 2010a; Privitera et al., 2010; Wierda et al., 2012) and response preparation and execution (e.g. Einhäuser et al., 2010; Hupé et al., 2009; Kloosterman et al., 2015; Privitera et al., 2014; Privitera et al., 2010; Richer \& Beatty, 1985). The current experiment used a task similar to that which 

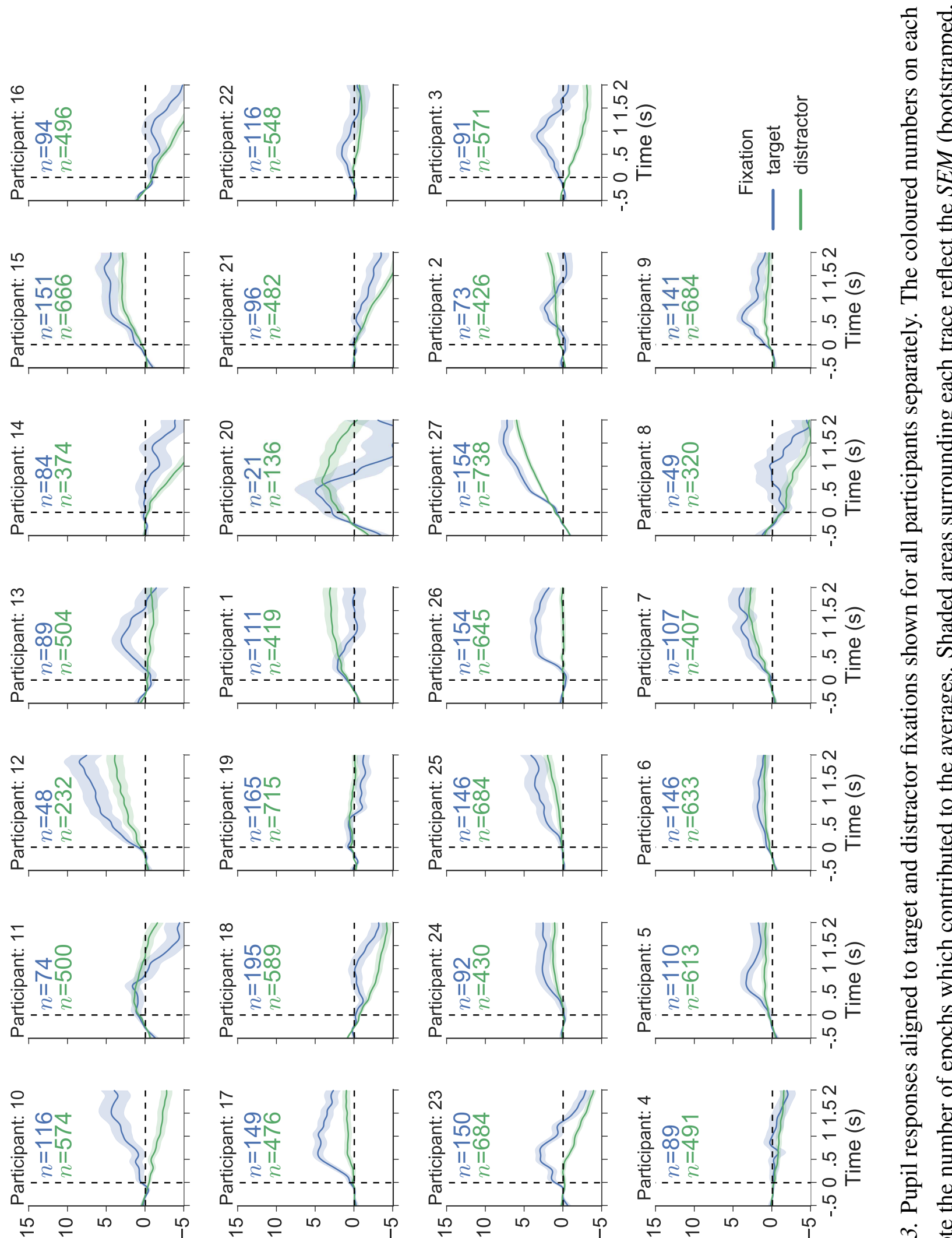

(\%) uo!̣ejnpom !!dnd (\%) uo!̣e|npom !!dnd

(\%) uo!̣ejnpom !!dnd (\%) uo!̣ejnpou !!dnd

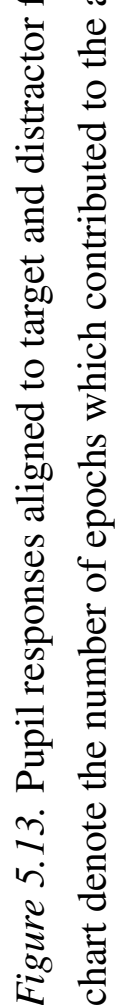




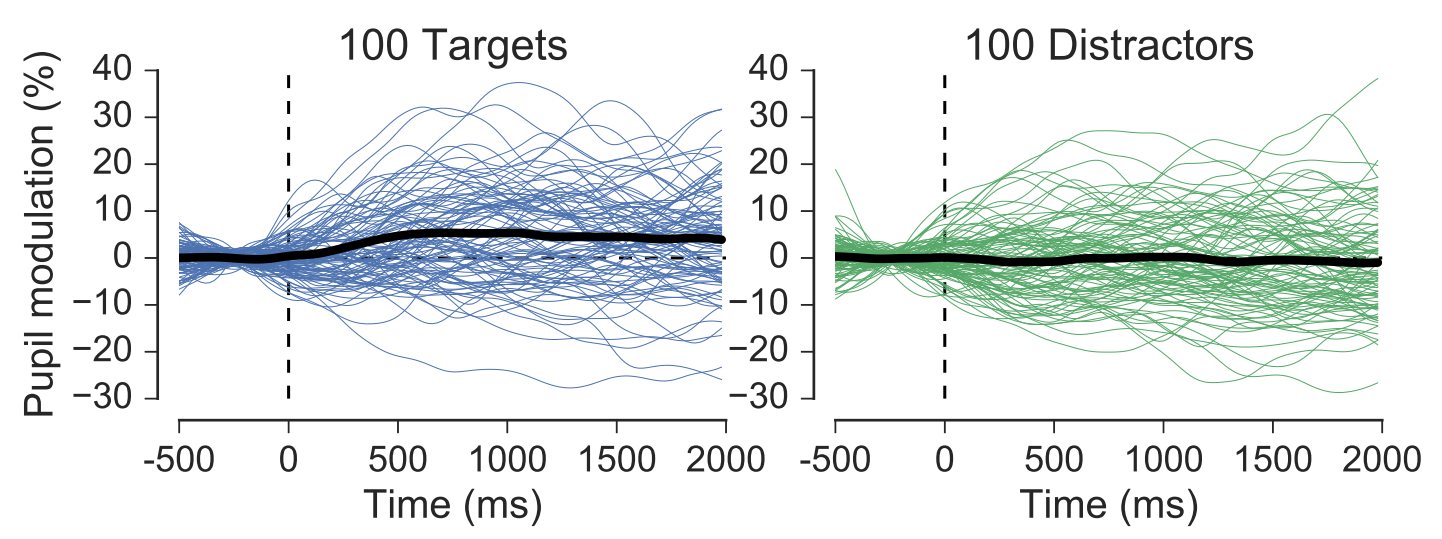

Figure 5.14. Single-trial variability for target and distractor fixations in one subject with prototypical averages (Participant 17). One hundred target and distractor epochs were chosen at random and visualised, with their respective averages shown in black.
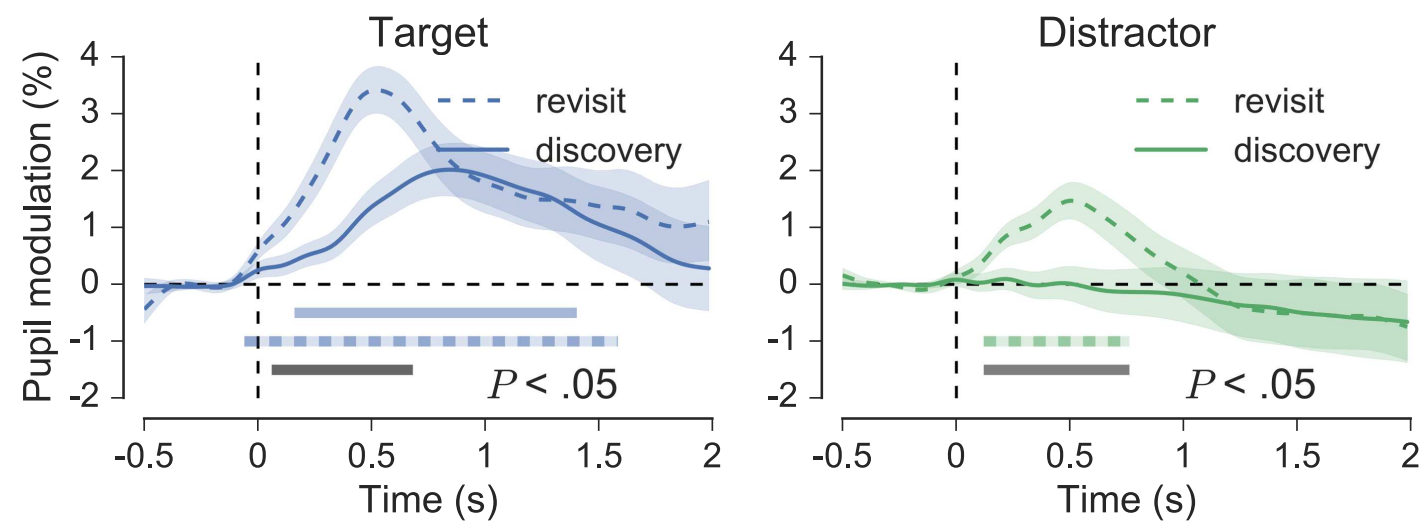

Figure 5.15. Grand-average pupil responses for target and distractor discoveries and revisits. Shaded areas surrounding each trace reflect the SEM (bootstrapped, 5000 iterations). Horizontal coloured bars indicate clusters of significant modulation from baseline and grey bars indicate significant differences between the coloured traces, as revealed by nonparametric permutation tests (1024 permutations, $p<.05$, clustercorrected for multiple comparisons). 


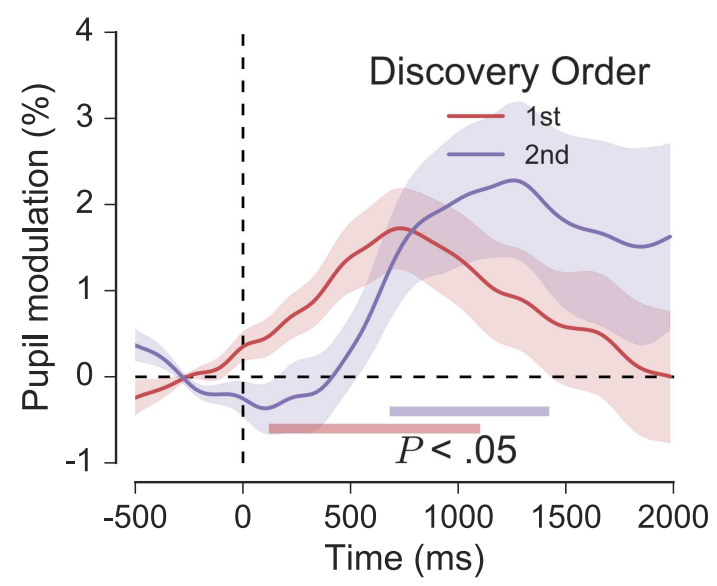

Figure 5.16. Fixation-aligned pupil responses for first and second target discoveries in trials with multiple targets. Shaded areas surrounding each trace reflect the SEM (bootstrapped, 5000 iterations). Horizontal coloured bars indicate clusters of significant modulation from baseline, as revealed by nonparametric permutation tests (1024 permutations, $p<.05$, cluster-corrected for multiple comparisons).

was used by Porter et al., the differences being that up to 3 targets could be present and participants could choose whether to terminate the search before they reported how many targets were present at the end of the trial via mouse-click. In addition to Porter et al.'s whole-trial approach to the analysis of pupil data, fixation-aligned pupillary response averaging (Klingner, 2010a) was used to explore changes in pupil size associated with task components during search. It was predicted that transient pupil dilations would coincide with target detection during search and with button presses to terminate search.

First, it should be noted that the performance measures are in keeping with findings from previous studies of multiple target search and with what can be expected given the demands of the task. Accuracy decreased as Targets increased, which can be explained in terms of the well-characterised phenomenon of subsequent search misses (Adamo et al., 2013; Biggs, 2017; Cain et al., 2013; Gorbunova, 2017, see also Section 2.3.3 of the current thesis). According to Cain et al. (2013), subsequent search misses arise primarily from scanning errors (i.e. failure to fixate the target during the search period), but are also associated with the cognitive effects of targets that have already been found (e.g. depletion of WM resources that could have been used for further search), and to a lesser extent, with recognition, decision and strategy errors. Also observed in the current experiment was a reduced search time and increased proportion of termination-searches for 3-target trials compared to all other conditions. These 
findings can be readily understood in terms of the task demands and the general strategy adopted by participants. It was known in advance that up to 3 targets could be present on every trial, meaning that accurate responses depended on finding either 3 unique targets or on exhaustively inspecting each item in the search array. If 3 unique targets were found before all of the items were inspected, participants could immediately terminate the search and provide the correct answer with relative confidence. However, without the discovery of a third unique target, accuracy always depended on a thorough inspection of the array, leading to longer search times and a reduced proportion of termination-searches.

The results of the whole-trial analysis of pupil data are now considered. In Porter et al.'s (2007) experiments, pupil size began to increase at the beginning of search, plateaued mid-search and then increased again towards the end of search when participants made a response-a pattern that was generally consistent with the findings from pupillometry experiments using tasks other than search up to that point (e.g. Beatty, 1982b; Hyönä, Tommola, \& Alaja, 1995; Kahneman, 1973). The same general pattern of search-related pupil size changes observed by Porter et al. was found in the present experiment, although pupil size decreased more dramatically after the initial peak, and only peaked at the end of search for 3-target trials. The large peak at the end of 3-target trials was clearly related to self-termination with a button response, which occurred more frequently in this condition (right-hand panel of Figure 5.8). To examine this finding further, the pupil data at search offset were grouped by whether the trial was self-terminated or not (Figure 5.11). Interestingly, the significant difference in pupil size between termination and nontermination searches appeared up to $250 \mathrm{~ms}$ before search offset. This pattern of results is strongly redolent of findings from an experiment by Einhäuser et al. (2010), where participants were required to press a button at any time during a $10 \mathrm{~s}$ period, and pupil size associated with the button response began to emerge up to $1500 \mathrm{~ms}$ prior to the event. Therefore, the present experiment provides support to Einhäuser et al.'s suggestion that pupil dilation may betray the timing of a decision before it is reported voluntarily. This pupillometric observation is similar in principle to the so called prereadiness potential, an ERP signal which begins to emerge before participants report becoming aware of an intention to press a button (Libet, Gleason, Wright, \& Pearl, 1983). Finally, although pupil size did appear to be larger on average for trials with more targets, this effect was not significant.

Whereas whole-trial analysis gave general insight into the patterns of pupil dilation observed across the task, it did not say anything about the pupil dynamics surrounding task components during search. The fixation-aligned pupillary response 
averages did however shed some light on this. Target fixation evoked brief transient pupil dilation (shown in Figure 5.12), similar to that which was observed by Klingner (2010a) with his original use of the method. This effect could not have been linked to display changes or button responses, because epochs were only analysed if the associated fixation did not fall within $1000 \mathrm{~ms}$ of the display change at the beginning of the experiment or $2500 \mathrm{~ms}$ of search offset (whether brought about by a button response or not). Also in line with Klingner's findings is the present finding that distractor fixation did not cause the pupil to dilate. This main finding of a difference in pupil modulation for target and distractor fixations was statistically significant at group-level for 26 participants - a considerable improvement on Klingner's original observations which were made across all epochs from 17 participants and not quantified statistically. Also noteworthy is that this finding was not dependant on correction for gaze position, and was in fact little changed for PFE-corrected and uncorrected pupil data (Figure 5.12). The effect of target vs. distractor fixation in the present experiment therefore provides further support for the validity of the fixation-aligned pupillary response averaging technique, and also corroborates findings from other pupillometry studies where effects of target detection were observed in the absence of a motor response (e.g. Privitera et al., 2010; Wierda et al., 2012).

The pupillometric responses to targets observed in the current experiment most likely reflect phasic activation of the LC in response to the discovery of task-relevant stimuli. Phasic responses in the LC following the detection of target stimuli have been observed directly with single unit recordings in nonhuman primates (e.g. Rajkowski et al., 2004), and some studies have even shown a tight correlation between this activity and concurrent measures of pupil size (e.g. Joshi et al., 2016). Noradrenalin released by the LC has a neoromodulatory role in cognition and perception, enhancing effects on attention, decision processes, memory and acuity (Aston-Jones \& Cohen, 2005; Bouret \& Richmond, 2009; Bouret \& Sara, 2005). Therefore it seems likely that phasic LC activation following target discovery in the current experiment, as revealed by transient pupil dilation, reflects cognitive processes linked to the encoding of target location and the update to the running count of targets in WM for report at the end of the trial.

In addition to replicating Klingner's (2010) observations, the current analysis aimed to provide more information on the reliability of the effects of target and distractor fixation at both the participant and single-epoch level. As can be seen in Figure 5.13, the majority of participants displayed larger responses to targets overall, but the characteristic peak for target fixation observed in the group-level average was not always present. At the single epoch level, pupil data were very noisy (shown in Figure 5.14), 
suggesting limitations on the practical utility of these findings. For instance, inferring target presence from individual epochs of pupil data would not be possible in the present experimental context. Much of the variance at the single trial level is likely to be due to gaze position artefacts that were not remedied by the PFE correction procedure. This contention is based on visual inspection of the individual epochs in Figure 5.14, whose erratic fluctuations in pupil size are uncharacteristic of normal pupillary behaviour for a cognitive task under conditions of constant luminance (see Loewenfeld, 1993; Mathôt et al., 2018).

Finally, Klingner (2010a) reported that pupil dilation began earlier when targets were revisited. In search of this effect, fixations were split into discoveries (initial fixations on a display item) and revisits (refixations on a display item). Target revisits evoked significantly larger pupil responses than target discoveries, and although dilation did not begin $500 \mathrm{~ms}$ before fixation, peak dilation was earlier on average (left panel of Figure 5.15). It is unclear what the cause of this difference should be. Klingner, who reported dilation up to $500 \mathrm{~ms}$ prior to the fixation, suggested the effect could be linked to saccade planning to reaffirm the location of a previously visited item. Clearly this explanation does not fit the present findings, where dilation begins only milliseconds before the fixation for targets. It could be that the larger dilations observed for target revisits reflect a cognitive mechanism associated with the awareness of having already counted the target, which maintains accuracy by ensuring that they are not added to the running total. However, given the similarity of the revisitation component in both targets and distractors with respect to time and magnitude, it is also possible that the larger pupil responses following revisitations were linked to systematic effects associated with fixation patterns or pupil foreshortening.

\subsection{Overall discussion}

The research presented in the current chapter confronted a range of methodological issues in an attempt to gain further insight into the cognitive pupil dynamics of free-viewing visual search. Experiment 3 demonstrated the PFE and how it can be ameliorated, albeit not completely removed, using an established data-driven regression procedure (Brisson et al., 2013). Experiment 4 used the regression models from Experiment 3 in order to correct for gaze position artefacts in a free-viewing visual search task, and also implemented two different yet complementary techniques for analysing the pupil data. Whole-trial analysis (Porter et al., 2007) was used to explore the general pattern of pupil size changes across the duration of the task. Though it did not suggest 
any robust cognitive effects arising from differences in the number of targets that were present in the search, it did provide general insight into search-related processing and helped to characterise the substantial effect of pupil dilation associated with an optional motor response. Fixation-aligned pupillary response averaging (Klingner, 2010a) was used to gain insight into the pupil dynamics surrounding fixations on targets and distractors during search whilst removing the confounds associated with display changes and button-pressing. The main finding to emerge through the use of this technique was that of small transient pupil dilations following fixations on targets, but not distractors. This effect was robust at the group-level, though it was less consistent at the level of individual participants, and even less so at the level of single fixation epochs.

From a purely practical perspective, a key message of the findings from the current chapter is that, although pupillometry in free-viewing visual search paradigms is not without complications, it is by all means possible and can lead to useful insights into search-related processes. The PFE was unquestionably a source of error in the data of Experiment 4 and was likely a substantial contributing factor to the noise observed at the level of single fixation epochs for the effect of target detection. However, the grouplevel statistical significance of the main experimental effect of target detection was not contingent on PFE correction, which suggests that the random positioning of search elements during stimulus preparation may have been a sufficient countermeasure per se. This implies that for the purposes of cognitive research where inferences are to be based on group-level statistics, it may not be necessary to correct for the PFE in free-viewing paradigms providing the locations of task-relevant stimuli are effectively randomised. It may also be possible to further discourage idiosyncratic viewing behaviour, such as the overall preference for a top-to-bottom search strategy observed in Experiment 4, by varying the locus of fixation at the start of each trial.

Overall, the present chapter has set out a strong methodological basis for pupillometric studies of free-viewing visual search. The synthesis of methods across both experiments lead to greater insights into search-related cognitive processing than the highly constrained approach of brief stimulus presentations and constant fixation that was used in the previous chapter. Future studies could combine alternative and more reliable methods of PFE-correction (e.g. model-based correction Gagl et al., 2011; Hayes \& Petrov, 2015) with whole-trial analysis, fixation-aligned pupillary response averaging, and equally mindful approaches to stimulus preparation and task design in order to address more detailed questions about the temporal dynamics of pupil-linked cognition in visual search. 


\section{Chapter 6}

\section{Pupil size and performance measures during vigilance tasks}

\subsection{General introduction}

In the previous two chapters, the experimental focus was on the cognitive pupil dynamics of visual search. The present chapter explores what eye tracking and pupil measures can reveal about cognitive processes involved in vigilance task performance. As discussed in detail in Section 2.2, vigilance tasks test one's ability to remain alert and respond to critical signals over prolonged periods of time, and have been used for over half a century to systematically study vigilant attention in a range of settings. To date, few studies have examined how pupil metrics relate to performance in vigilance tasks, but there is a broad range of evidence linking changes in pupil size to sleepiness, arousal, fatigue and activity in the LC-NA system - all of which have been associated with the vigilance decrement (e.g. Crawford, 1936; Hopstaken, van der Linden, et al., 2015; Hou et al., 2005; Joshi et al., 2016; Loewenfeld, 1993; Lowenstein et al., 1963; Lowenstein \& Loewenfeld, 1964; Phillips et al., 2000; Rajkowski et al., 1993; Varazzani et al., 2015; Yoss et al., 1970a, 1970b, see also Sections 2.2.4, 2.5.4 and 2.5.5 of the current thesis). A more detailed knowledge of how pupil responses vary throughout vigilance tasks and how they relate to task performance measures will aid future research efforts and also help to establish the practical potential for using pupil measures to asses vigilance in operational environments.

The two main genres of task outlined in Chapter 2.2.1 were standard vigilance tasks and psychomotor vigilance tasks (PVT), both of which represent different approaches to the measurement of vigilant attention. Standard vigilance tasks generally aim to explore vigilant attention by simulating the conditions of real world scenar- 
ios where monotonous repetitive tasks have become commonplace due to industrial mechanisation, a classic example being the Mackworth clock-procedure (Mackworth, 1948), which lead to the discovery of the vigilance decrement (note Section 2.2.1). Although standard vigilance tasks can vary in many ways, their main characteristic is that observers must remain alert and respond to critical signals presented against a background of noncritical signals over prolonged, unbroken stretches of time, usually at least 30 min (Frankmann \& Adams, 1962; Parasuraman \& Davies, 1976). PVTs on the other hand require observers to make speeded responses to more frequent signals occurring at random intervals over short periods of time, and are usually administered repeatedly to measure changes in vigilant attention arising from various environmental stressors, such as time spent on-task and sleep deprivation (Dinges et al., 1997). Both types of task are reviewed again here, together with the pupillometric research that has been conducted with them.

\subsubsection{Pupillometry and PVTs}

Since their conception, PVTs have been used to assess vigilant attention in clinical, operational and research environments (Dorrian, Rogers, \& Dinges, 2005), and have become one of the most widely used neurobehavioural tests in circadian rhythm and sleep research (e.g. Basner \& Dinges, 2011; Basner et al., 2011; Chua et al., 2012; Dinges et al., 1997; Drummond et al., 2005; Graw et al., 2004; Roach, Dawson, \& Lamond, 2006; Van Dongen \& Dinges, 2005). The standard format is a 10 min task recording RTs to simple visual (sometimes auditory) stimuli which occur at random inter-stimulus intervals (ISI), usually between 2-10 s (Dinges \& Powell, 1985; Dorrian et al., 2005). A broad range of outcome metrics can be derived from the data, but performance is most commonly assessed by examining mean RTs and the number of lapses, which are normally defined as RTs greater than $500 \mathrm{~ms}$ or twice the mean RT (Basner \& Dinges, 2011). Declines in vigilant attention result in an overall slowing of RTs and a gradual increase in the number of lapses across repeated task administrations. Such effects are reliably produced by sleep deprivation and increased time spent on-task (Gunzelmann et al., 2009).

PVTs are often used in field studies where an experimenter collects data from many participants under various conditions for later analysis. In these instances, the task is usually administered via dedicated hardware, such as the PVT-192 (e.g. Loh, Lamond, Dorrian, Roach, \& Dawson, 2004; Roach et al., 2006) or the Palm-PVT (e.g. Thorne et al., 2005). These devices are portable all-in-one solutions to presenting stimuli, recording responses and storing data. Although the stimulus can vary between 
devices, the prototypical stimulus is the onset of a 4-digit millisecond counter displayed on an LED screen (e.g. Dinges \& Powell, 1985; Wilkinson \& Houghton, 1982). The counter is fixed at '0000' between trials but begins to increment every 2-10 s, prompting the participant to respond. Once the response is detected, the counter freezes and after a short while resets to zero. When PVTs are used in research laboratories they are often administered on a standard computer, with stimuli presented on a monitor and responses recorded on a keyboard, mouse or dedicated response box (e.g. Basner \& Dinges, 2011).

Declining PVT performance has been linked to various physiological measures such as increasing theta activity in the EEG (Caldwell et al., 2003), increasing heart rate variability (Chua et al., 2012) and increasing fMRI activation in the 'default mode network' (Drummond et al., 2005) —all of which are known to reflect autonomic arousal. A handful of studies have also explored how changes in pupil size relate to PVT performance. One study by Kristjansson et al. (2009) examined pupil responses in an 'enhanced' PVT, where participants had to respond to a running counter every 1.5 to 3.5 s continuously for an hour ${ }^{1}$. Pupil data from $102 \mathrm{~ms}$ before response onset to $918 \mathrm{~ms}$ after the response were extracted for temporally adjacent normal-latency responses (selected from the midrange RTs) and long-latency responses (selected from the 50 slowest RTs), and then individually parametrised into measures reflecting average pupil diameter, linear dilation rate and curvilinear dilation rate. Two key findings emerged from this experiment. First, prestimulus baseline pupil diameter was significantly greater for normal-latency responses compared to long-latency responses, suggesting that longer RTs were associated with lower alertness at the time of stimulus onset. Second, after controlling for the differences in prestimulus baseline, the Yintercepts, linear change rates and curvilinear change rates were all significantly greater for long-latency responses than for short-latency responses, with linear slopes (reflecting dilation velocity) differing as early as $264 \mathrm{~ms}$ after response onset. Although there was considerable variation at the level of individual subjects, the general observations implied that real-time parametrisation of pupil data following detection responses could serve as a method for assessing levels of alertness on a moment-to-moment basis.

McClelland et al. (2010) conducted a study exploring how various oculomotor measures relate to performance on cognitive tasks under conditions of sleep deprivation. Participants completed 5 testing sessions throughout a night of wakefulness, with each session involving an oculomotor assessment and a cognitive testing battery. The

\footnotetext{
${ }^{1}$ Given its length and uninterrupted nature, this task could also be thought of as a standard vigilance task with a high event rate. However, it is reviewed here due its use of the running counter stimulus and variable ISI, which are characteristic of PVTs.
} 
oculomotor assessment was conducted at the beginning of each session and included measurements of initial pupil diameter, constriction amplitude and constriction latency in response to a light stimulus. The cognitive tests involved a standard PVT task administered via a PVT-192 device (e.g. Loh et al., 2004; Roach et al., 2006). Pupil size was not recorded during PVT task administration, but declining performance across the 5 sessions was significantly predicted by two of the pupil measures. Specifically, initial pupil diameter and constriction latency were negatively associated with PVT performance, such that a $1 \mathrm{~mm}$ decrease in initial pupil diameter was associated with a 21 $\mathrm{ms}$ increase in RT on the PVT, and a $1 \mathrm{~ms}$ decrease in constriction latency was associated with a $23 \mathrm{~ms}$ increase in RT. These findings suggest that repeated measurements of pupil diameter and constriction latency may serve as a useful index of declines in PVT performance.

A recent study by Unsworth and Robison (2016) has linked pupil measures to both lapses in attention on a PVT and self-reported attentional states. Participants responded to a running counter stimulus every $2-10 \mathrm{~s}$ in 5 contiguous blocks of 32 trials, and also responded to random thought probes that were inserted after some trials to indicate their mode of thought prior to the appearance of the probe (e.g. task-related thought, mind-wandering). PVT performance was measured in terms of mean RTs, number of lapses and the standard deviation of RTs, all of which trended significantly upwards across each block. Analysis of the thought probe responses indicated that reports of mind-wandering increased across blocks and reports of being on-task decreased. The results from the pupillometry analysis showed that pretrial baseline pupil diameter as well as task-evoked responses decreased with time spent on-task. Additionally, RTs were longer and both pretrial baseline pupil diameter and task-evoked responses were smaller for trials when participants reported being off-task at the thought probe. These findings imply that fluctuations in pupil size track the attentional state of an observer, and may therefore serve to indicate when lapses of attention are most likely to occur.

Massar, Lim, Sasmita, and Chee (2016) conducted two pupillometry experiments where participants performed a PVT in various reward conditions. In the first experiment, participants completed three runs where they could earn either 0,1 or 10 cents for fast responses (i.e. baseline, low and high reward). Each run lasted 6 min and the stimulus was a running counter with onsets ranging between 2-12 s. Reward had a significant effect on the number of lapses and RTs, both of which decreased as the reward increased. Additionally, prestimulus pupil diameter increased significantly as a function of reward, suggesting that the prospect of reward is associated with a higher level of arousal or attentional effort. The findings were replicated in another experiment where the 
low-reward condition was substituted for a random reward condition (instead of low reward, participants were told they would receive 10 cent rewards at random times, not contingent upon their performance). In this experiment, an increase in pupil diameter was only present when rewards depended on good task performance. Additionally, in both experiments, prestimulus pupil diameter steadily decreased as a function of time spent on-task, which corroborates the findings of Unsworth and Robison (2016). Overall, these findings are evidence that PVT task performance is improved when greater reward is anticipated, and that this improvement is associated with changes in prestimulus pupil size.

\subsubsection{Pupillometry and standard vigilance tasks}

In the present chapter, standard vigilance tasks are defined as those which require sustaining attention for prolonged, unbroken stretches of time-at least $30 \mathrm{~min}$-in order to respond to critical signals presented against a background of noncritical signals (Frankmann \& Adams, 1962; Parasuraman \& Davies, 1976). The Mackworth Clock Test, described in Section 2.2.1, was the first example of a standard vigilance task used in an experimental setting. Participants watched a black pointer on a blank background as it ticked like the second hand of a clock and had to respond by pressing a switch every time they noticed the pointer move twice the normal distance, which happened 12 times every $30 \mathrm{~min}$ for the $2 \mathrm{hr}$ duration of the task. The task was designed to simulate the conditions of radar and sonar operators during World War 2, who often failed to detect weak signals on their displays signifying the presence of enemy submarines. In Mackworth's experiment, detection accuracy declined by $10 \%$ to $15 \%$ after only 30 min of the task had elapsed, and then continued to decrease gradually for the remainder of the task, suggesting that vigilant attention wanes rapidly during periods of prolonged monitoring. This finding has since been replicated extensively and is now widely referred to as the vigilance decrement (Parasuraman \& Davies, 1982; Parasuraman et al., 1998; Warm \& Jerison, 1984; Warm et al., 2008).

Mackworth's study aroused considerable interest in the scientific exploration of vigilance and gave rise to a large body of empirical work in the domains of human factors and experimental psychology. Human factors researchers began to use vigilance tasks as a means of gaining insight into human performance in operational environments (Adams, 1987; Craig, 1985; Mackie, 1987; Wiener, 1984, 1987), whereas experimental psychologists used them as a method of exploring the various factors that influence human attention (Broadbent, 1958; Broadbent \& Gregory, 1965; Dember \& Warm, 1979). The types of vigilance task that have been used in experimental work are many 
and varied. Key differences between tasks which influence performance are the sensory modality of stimulus presentation (e.g. auditory, visual), the psychophysical dimensions used to define the critical signals (e.g. brightness, loudness), and whether the detection of targets requires successive or simultaneous discrimination ${ }^{2}$ (Warm et al., 2008); but performance ultimately depends on complex interactions of various task-related, environmental and individual factors (Ballard, 1996), many of which were outlined in Section 2.2.2.

Many studies have explored neural and physiological correlates of declining vigilance performance (for review see Fortenbaugh et al., 2017; Oken, Salinsky, \& Elsas, 2006, also see Section 2.2.4), but only a handful of experiments to the authors knowledge have explored how pupil measures relate to performance in a standard vigilance task. The earliest known example is a study by Beatty (1982a), where participants were asked to monitor a string of tones at $3.2 \mathrm{~s}$ intervals continuously for $48 \mathrm{~min}$ and detect target tones, which differed with respect to the nontargets in that they were slightly attenuated in volume. Approximately 12 targets were presented at random intervals every $5 \mathrm{~min}$, with 108 targets being presented across the whole task. Performance was uninterrupted for the full duration of the experiment, but for the purposes of analysis, the data were averaged within thirds of the test. Detection accuracy decreased in accordance with time spent on task, replicating the classic vigilance decrement findings discussed previously in Section 2.2.1. Interestingly, evoked pupillary responses to target stimuli mirrored these results, decreasing in amplitude across each third of the test, but tonic pupillary measurements (obtained prior to each target stimulus) were relatively unchanged. This implied that declining performance may have been linked to gradual disengagement with the task as opposed to an overall reduction in arousal levels, which is consistent with a mind-wandering account of the vigilance decrement (note Section 2.2.3).

Gilzenrat et al. (2010) conducted a series of experiments using tasks similar to the task used by Beatty (1982a). Participants were presented with $250 \mathrm{~ms}$ tones every 2.5 to $5.2 \mathrm{~s}$ continuously for $50 \mathrm{~min}$ and had to respond via keypress to target tones (880 $\mathrm{Hz}, 20 \%$ prevalence) and ignore distractor tones $(800 \mathrm{~Hz}, 80 \%$ prevalence). Focusing closely on the relationship between baseline pupil size, stimulus-evoked responses and performance measures, Gilzenrat et al. found that larger pupils at baseline ( 75 th

\footnotetext{
${ }^{2}$ The distinction between successive and simultaneous vigilance tasks was first made by Parasuraman (1979). Successive tasks are absolute judgement tasks where observers must compare the current sensory input with a template in WM in order to determine whether a particular stimulus is, or is not, a critical signal. Simultaneous tasks on the other hand are comparative judgement tasks, where each stimulus contains all of the information required to determine whether it is (or is not) a signal. Due to the involvement of WM, successive tasks are thought to be more resource demanding than simultaneous tasks.
} 
percentile) were associated with increased errors, longer RTs and reduced stimulusevoked dilation, whereas smaller pupils (25th percentile) were associated with the opposite pattern of results. These findings were taken as evidence for the adaptive gain theory of LC-NA function which proposes that exploratory and exploitative behavioural states are associated with tonic and phasic modes of LC function, respectively (AstonJones \& Cohen, 2005, also see Section 2.5.3). These findings were partially replicated by Murphy et al. (2011), who also reported that stimulus-evoked responses decreased in amplitude across time-on-task in an auditory oddball paradigm, but that baseline pupil diameter increased. This latter finding of increasing baseline pupil diameter was unusual, but the authors pointed out that performance was at ceiling throughout the task, and suggested that the gradual increase in pupil diameter may have been associated with increasing distractedness as participants learned to maintain optimum performance with a diminishing need for task engagement.

Finally, there is only a single study to the author's knowledge which examined pupil and performance measures in a standard vigilance task where critical signals were presented in the visual modality. McIntire, McKinley, and Goodyear (2014) asked participants to monitor a simulated air-traffic control display consisting of 4 triangular jet icons in four separate quadrants. The icons were presented at random going either counterclockwise or clockwise along a flight path and participants had to respond to rare occasions where one of the icons was oriented in the opposite direction to all of the others. The task lasted for $40 \mathrm{~min}$ and was completed 4 times by each participant on successive days. McIntire, McKinley, and Goodyear observed a classic vigilance decrement in performance across each 10 min time-on-task block, and found also that average pupil size decreased in a similar fashion. This study did not examine event-related pupil responses, but it provides support for the general observation of the cooccurrence between decreasing pupil size and declining vigilance performance. It is unclear to what extent the pupil data were affected by the physical characteristics of the stimuli used in this study, as very little methodological information was given in this regard.

\subsubsection{Other relevant findings}

The previous two sections have considered findings from experiments that measured pupil size and behavioural performance in tasks that could be classified as either a PVT or a standard vigilance task - the two main forms of vigilance task that are of interest in the current chapter. The general distinction between these two types of task is useful, as they each represent different yet firmly established approaches to the measurement 
of vigilant attention. However, the distinction is somewhat limiting in that it precludes the consideration of many other relevant findings from pupillometry experiments with tasks that require vigilance but are not PVTs or standard vigilance tasks per se. This section therefore reviews a selection of relevant findings from such experiments.

Van Orden et al. (2000) conducted a study to explore how various ocular measures, including pupil size, relate to performance on a sustained visual compensatory tracking task. Participants completed two 53 min sessions of the task, which involved using a trackball to move a target disk (destabilised by random directional movements) into the middle of an annulus on a CRT screen. Error, which was defined as the distance of the disk from the centre of the annulus, was measured continuously throughout the task in disk-radius units. On average, tracking error increased at the start of each session, reached peak after around $11 \mathrm{~min}$, and then decreased steadily across the remaining duration. Throughout the task, pupil size covaried with performance such that periods of poor performance were associated with smaller pupils and periods of better performance with larger pupils.

Some experiments have explored how fluctuations in pupil size relate to attentional decoupling or task-unrelated-thought, which according to underload theories of vigilance are important determinants of task performance (note Section 2.2.3). Smallwood et al. (2011) compared performance and pupil measures for two versions of a probe detection task, each requiring different modes of cognition. In both tasks, participants were presented with a stream of noncoloured digits for $20 \mathrm{~min}$ and had to respond to coloured probes at variable intervals. In the easier of the two tasks the probe was a coloured digit and participants simply had to indicate whether it was odd or even, meaning accurate performance did not depend on sustained attention to the noncoloured digits. In the more difficult task the probe was a coloured '?' and participants had to indicate whether the previous digit was odd or even, meaning they had to continuously monitor the noncoloured digits in order to perform accurately. Smallwood et al. argued that these tasks were conducive to offline and online modes of thought ${ }^{3}$, something which was confirmed in an initial experience sampling experiment where participants reported being more focused on the past or the future in the easy task, but more focused on the 'here and now' in the difficult task. Pupil diameter, which was recorded throughout each task, exhibited different patterns of activity for task periods and events conducive to online and offline thought. When cognition was online (i.e.

\footnotetext{
${ }^{3}$ Throughout the mind-wandering literature researchers frequently distinguish between online and offline cognition (e.g. Mittner et al., 2014; Smallwood et al., 2011; Smallwood \& Schooler, 2015). During online cognition attention is coupled to perceptual input and sensory events receive enhanced processing, whereas in offline cognition attention is decoupled from perception and internally generated events receive enhanced processing.
} 
when probes were responded to correctly in both tasks) the pupil responded phasically to task relevant stimuli; but when cognition was offline (i.e. for nonprobe stimuli in the easy task and when responses were incorrect in both tasks), the pupil showed high baseline activity and a lack of responsiveness to external stimuli. These findings were taken as support for the decoupling hypothesis of spontaneous thought (e.g. Corbetta \& Shulman, 2002; Raichle, 2010), and together with the findings of subsequent similar studies (e.g. Franklin, Broadway, Mrazek, Smallwood, \& Schooler, 2013; Grandchamp, Braboszcz, \& Delorme, 2014; Smallwood et al., 2012) they suggest that fluctuations in pupil size may serve as an index for changes in attentional state.

Pupillometry has also been used in conjunction with self-report measures to explore mental fatigue in long and demanding tasks. Hopstaken and colleagues (Hopstaken, Van der Linden, Bakker, \& Kompier, 2015; Hopstaken, Van der Linden, Bakker, Kompier, \& Leung, 2016; Hopstaken, van der Linden, et al., 2015) have conducted a collection of experiments where participants had to perform for 2 hours on a visual letter $n$-back task, which required continuous engagement of attention and WM. Participants were presented with letters for $500 \mathrm{~ms}$ every 5 to $5.5 \mathrm{~s}$ and had to indicate whether the letter was a target or a nontarget ${ }^{4}$. Between each block, participants stopped briefly to indicate their current level of task engagement and mental fatigue using a visual analogue scale. In all of the experiments, performance, self-reported attention to the task and baseline pupil size decreased steadily across each time-on-task block. However, before the final block participants were told that the remaining length of the experiment would depend on their performance (it would be shorter if performance was better), which caused performance and pupil measures to return to the levels observed at the beginning of the task. Therefore, as well as providing strong evidence for the covariance between pupil size, task performance measures and mental fatigue, the experiments of Hopstaken and colleagues also show how a reward manipulation can restore pupil size to prefatigue levels.

The final example to be considered is a recent study by van den Brink et al. (2016), which explored pupil size measures during a gradual continuous performance task. Participants had to respond with a keypress to pictures of cities (90\% of trials) and withhold responses to pictures of mountains (10\% of trials) for $8 \mathrm{~min}$ at a time. The images were greyscale and morphed linearly and continuously into one another with $100 \%$ coherence occurring every $800 \mathrm{~ms}$, meaning continuous attention was required and participants could not take 'mini-breaks' between trials. van den Brink et al.

\footnotetext{
${ }^{4}$ In an $n$-back task, a stimulus is a target if it is identical to the stimulus presented $n$ letters previously. The task becomes harder as $n$ increases, because it is necessary to retain more information in WM in order to perform accurately.
} 
initially observed typical time-on-task effects across the 8-min duration of each task (participants completed 3 blocks with a break in between), whereby baseline pupil diameter and behavioural performance decreased gradually. However, after regressing out the time-on-task effects from the pupil data, the linear relationships disappeared and consistent inverted U-shaped trends emerged, such that longer RTs and accuracy errors occurred when the pupil was both relatively large and small. This finding was discussed in relation to the classic Yerkes-Dodson law (Yerkes \& Dodson, 1908) of optimal arousal for task performance, which is the widely observed phenomenon that both over- and under-arousal can lead to poor task performance.

\subsubsection{Summary and current direction}

Overall, the research outlined in the previous three sections provides strong evidence that pupillometry can be used to gain insight into an observer's attentional state during long and demanding tasks, and that changes in pupil size often reflect changes in behavioural performance. However, a closer look at the findings reveals a lack of consistency regarding the relationship between pupil size and other measures. Some studies reported that moments of off-task thought or poor performance were associated with large pupils at baseline (Franklin et al., 2013; Smallwood et al., 2012; Smallwood et al., 2011; Unsworth \& Robison, 2016), whereas others reported that poor task performance was associated with smaller pupils at baseline (Grandchamp et al., 2014; Hopstaken, Van der Linden, Bakker, \& Kompier, 2015; Kristjansson et al., 2009; Mittner et al., 2014; Van Orden et al., 2000), or that it occurs after a gradual decrease in pupil size (Grandchamp et al., 2014; McIntire, McKinley, \& Goodyear, 2014; Murphy et al., 2011). Further, poor task performance was also found to occur with both relatively large and small baseline pupil size within experiments (Murphy et al., 2011; Smallwood et al., 2012; Unsworth \& Robison, 2016; van den Brink et al., 2016), and one experiment even reported increasing prestimulus pupil diameter as a function of time-on-task in a 37-min auditory vigilance task without breaks (Murphy et al., 2011).

The above discrepancies are most likely due to the interplay of various factors relating to differences in methodology among the discussed studies. For instance, there was considerable variability in how performance was measured. Some studies focused primarily on RT measures, such as mean RTs (McClelland et al., 2010; Smallwood et al., 2012; Smallwood et al., 2011), a fraction of the slowest or fastest RTs (Kristjansson et al., 2009; Unsworth \& Robison, 2016; van den Brink et al., 2016), or RT variability (Murphy et al., 2011); whereas others focused more on perceptual sensitivity (i.e. $d^{\prime}$ : Beatty, 1982a; Hopstaken, Van der Linden, Bakker, \& Kompier, 2015; Hopstaken et al., 2016; 
Hopstaken, van der Linden, et al., 2015) or self-reported measures of task engagement (Franklin et al., 2013; Grandchamp et al., 2014; Mittner et al., 2014). Task demands also varied considerably across experiments, with some requiring only simple detection of stimuli (e.g. PVTs) and others requiring simultaneous (Beatty, 1982a; Gilzenrat et al., 2010; Murphy et al., 2011; van den Brink et al., 2016) or successive (Hopstaken, Van der Linden, Bakker, \& Kompier, 2015; Hopstaken et al., 2016; Hopstaken, van der Linden, et al., 2015; Smallwood et al., 2012; Smallwood et al., 2011) discrimination (see Footnote 2). Finally, a range of stimuli were used, and each may have had unique behavioural and / or pupillometric consequences. For example, the running counter stimulus used in all of the discussed PVT experiments provides feedback which could enable participants to detect declines in their performance and adopt compensatory strategies (Thorne et al., 2005). Also, for studies using visual stimuli, differences in luminance, colour and contrast may have contributed to pupillometric and behavioural variance.

Clearly there is a requirement for further research to focus on the relationship between pupil and performance measures in visual vigilance tasks. Ideally, such research should adhere to well established vigilance task frameworks and use equiluminant, low-contrast, monochrome stimuli. Such an approach would ensure that performance measures have a broad basis for comparison in the vigilance literature and that interpretations of pupil data are not complicated by stimulus-related factors.

\subsection{Experiment 5}

\subsubsection{Introduction}

Experiment 5 sought to further explore how pupil measures relate to PVT task performance, but with a novel pupillometry-optimised stimulus approach. All of the experiments outlined in Section 6.1.1 used the prototypical stimulus of a running millisecond timer that counts up from zero, but as pointed out by Thorne et al. (2005), this may have undesirable consequences. From a behavioural and pupillometric perspective, the two most important points made by Thorne et al. are as follows. First, the intensity of the stimulus changes in a nonlinear fashion as the running counter increases, which could be a source of increasing variance in both behavioural and pupil measures. Second, the counter provides feedback to participants whether feedback is desired or not, which may enable them to monitor their own performance during the session and increase attention or effort to compensate for a noticed decline-a boon which would typically not be available in operational settings. To avoid these issues Thorne et al. 
devised a version of the PVT with a luminance-based graphic stimulus comprising two alternating black and white circular annuli, resembling a target or bull's eye. This PVT was administered on a dedicated hand-held device, much like the PVT-192, and produced highly comparable results despite the different stimulus characteristics.

Because a luminance-based stimulus such as that used by Thorne et al. (2005) would pose problems for pupillometry, the RT-initiating stimulus opted for in the present PVT experiment was a change in the orientation of a low-contrast gabor patch. Participants were instructed simply to continually monitor a gabor patch at the centre of a screen and respond as quickly as possible by pressing the space bar every time it flipped on its side (i.e. when it rotated $90^{\circ}$ ). As in other PVTs, the vigilance element of the task was instantiated with time-on-task ( 13 min) and ISI (4-12 s) parameters. Due to the use of a lower intensity stimulus, it was expected that RTs in the current PVT would be slower on average than for PVTs using a running counter stimulus. However, because the current stimulus approach avoids the confounds of variable stimulus intensity and feedback, behavioural and pupil measures should more faithfully reflect changes in vigilant attention. Based on the general findings outlined in Section 6.1.1, it was predicted that time-on-task, both within and between successive blocks of the PVT, would lead to declining performance and a decrease in pupil size. Also, following the findings of Kristjansson et al. (2009) and Unsworth and Robison (2016), it was predicted that worse performance would be associated with smaller pupils at baseline.

\subsubsection{Method}

\section{Participants}

Twenty-five participants (18 females; age range 18 -36 years, $M=22.96, S D=4.65$ ) completed the experiment voluntarily or in exchange for course credit. All participants were students at Swansea University reporting normal or corrected-to-normal acuity and colour vision. The experimental protocol was approved by the Ministry of Defence Research Ethics Committee and the Department of Psychology Ethics Committee at Swansea University. Written informed consent was obtained from each participant.

\section{Design}

Performance and pupil measures were analysed in a repeated measures design as a function of Trial Group (1, 2, 3, 4, 5: sets of 18 contiguous trials within a PVT block) and Block (1, 2, 3: successive blocks of 90 trials), two factors which aimed to capture 


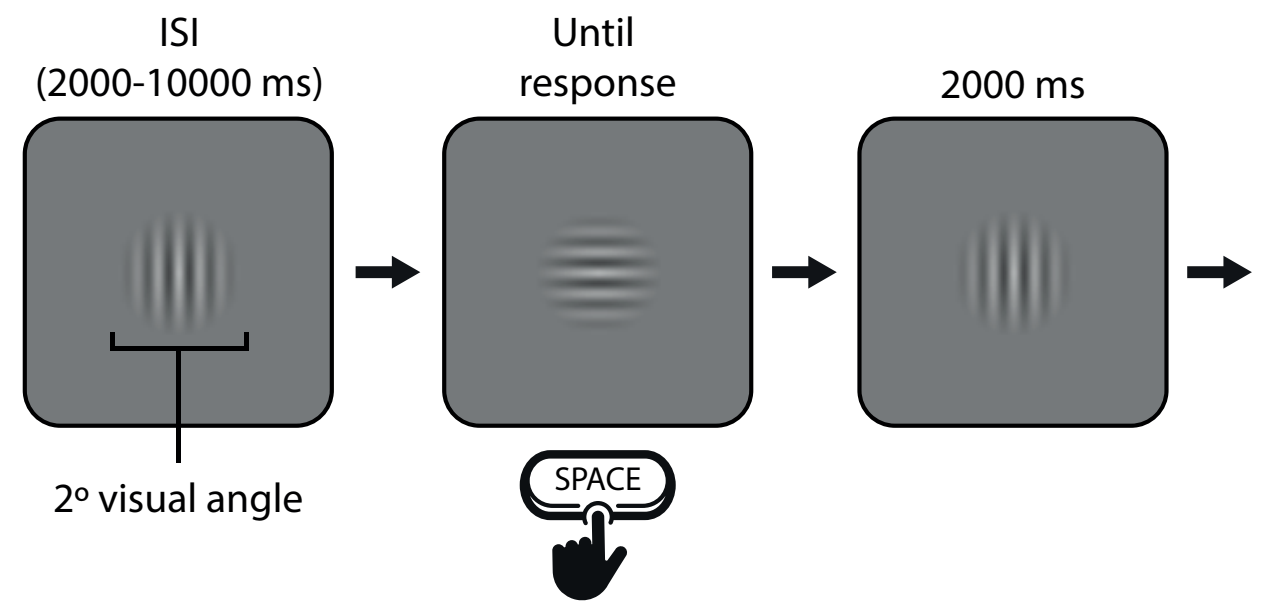

Figure 6.1. The stimuli and trial sequence for Experiment 5. Participants monitored a gabor patch and responded by pressing space every time it flipped on its side, which happened every 4-12 s.

within- and between-block experimental effects. Participants completed 3 blocks of the PVT in a single testing session, taking a forced break of only 1 min between blocks. In each block, participants monitored a gabor patch at the centre of a screen with the instruction to respond as quickly as possible by pressing the space bar whenever the gabor patch flipped $90^{\circ}$ on its side. The ISI, defined as the period between the last response and the next flip of the gabor patch, varied between 4-12 s. This period included a fixed component of $2000 \mathrm{~ms}$ and a random component varying between 2-10 s. The random component was constrained such that a third of the intervals would be short (2000-4666 ms), a third medium (4666-7333 ms), and a third long (7333-10000 $\mathrm{ms})$. Each block lasted approximately $13 \min (M=12.8, S D=0.63)$, with some small variability arising from differences in RT and the random element of the ISI. Continuous recordings of gaze position and pupil data were obtained for each trial, and RT was defined as the time from the flip of the gabor until the key response in milliseconds. The task was performed in a dimly lit room.

\section{Stimuli and apparatus}

The stimulus was a gabor patch enveloped within a cosine window (spatial frequency $=0.1, S D=12,39 \%$ contrast) presented at the centre of the screen, spanning $2^{\circ} \times$ $2^{\circ}$ of visual angle. It was generated online at https://www.cogsci.nl/gabor-generator and saved in JPEG format. The PVT was administered on a 24-in. Ilyama monitor running at a resolution of $1024 \times 768$ with a refresh rate of $144 \mathrm{~Hz}$ and responses were collected on a standard computer keyboard. The monitor and eye tracker were 
enclosed such that the only direct illumination came from the display screen. The screen was viewed from a distance of $40 \mathrm{~cm}$, which was maintained by a chin rest and forehead bar. Using a colorimeter (ColorCAL MKII, Cambridge Research Systems), the surface luminance of the grey background was recorded as $73.54 \mathrm{~cd} / \mathrm{m}^{2}$, and the surrounding dark light of the unused portion of screen as $0.53 \mathrm{~cd} / \mathrm{m}^{2}$. Pupil size and gaze data were recorded monocularly (left eye) using an EyeLink 1000 (SR Research, Mississauga, Ontario, Canada) system in tower mount configuration sampling at 1000 Hz. Eye level and camera position remained constant throughout the recording session for each participant. Stimulus presentation was managed with Experiment Builder (SR Research, Mississauga, Ontario, Canada).

\section{Procedure}

Participants were instructed to monitor the 'circular stimulus' at the centre of the screen and respond as quickly as possible by pressing the space bar whenever the patch flipped on its side, in order to reset it to its original position. These instructions were given in writing at the start of the testing session, together with graphic explanations of the stimulus event and the required action. Participants completed three consecutive blocks, taking a forced break of 1 min between each. A 5-point calibration and validation routine was performed at the start of each block.

\section{Performance and pupil measures}

The behavioural outcome measures used to assess performance in the present task were the mean number of lapses and the mean reciprocal transformation of RT (i.e. 1/RT). These are among the most commonly utilised outcome metrics in the PVT literature, and they have been reported to be among the most sensitive to declines in PVT performance arising from time spent on task (Basner \& Dinges, 2011). Lapses are most commonly defined as RT $\geq 500$ ms (e.g. Basner \& Dinges, 2011; Dorrian et al., 2005; Loh et al., 2004; Van Dongen \& Dinges, 2005), but due to the novel stimulus approach in the present PVT and between-participant variability in RT, lapse thresholds were set individually for each participant as RT $\geq$ twice their median RT, an approach similar in principle to that used by Basner et al. (2011). The reciprocal transform (1/RT) emphasises slowing in the intermediate and optimum response domains and markedly reduces the contribution of long lapses (Basner \& Dinges, 2011). This metric was calculated by dividing each RT (ms) by 1000 , taking the reciprocal transform, and then averaging. It is important to note that the scale of $1 / \mathrm{RT}$ is reversed in comparison to standard RT, such that an increase in 1/RT constitutes an improvement in performance 
and a decrease in $1 / \mathrm{RT}$ constitutes a decline. Infrequent responses without a stimulus or those with RTs $\leq 100 \mathrm{~ms}$ were counted as false starts (errors of commission) and removed from all analyses.

The pupil measures used in the current experiment were baseline pupil size, defined as the average pupil size in the $500 \mathrm{~ms}$ period immediately prior to stimulus onset ( $z$-scored across the whole experiment) and evoked responses, defined as the average pupil modulation in the $1500 \mathrm{~ms}$ period after the response, expressed as percent change from the prestimulus baseline. Time-locking pupil data to response events rather than critical stimulus events ensures that the motor component, which is an inevitable feature of a PVT, contributes consistently to pupil modulation for each trial. To further explore how both pupil measures relate to the extremes of behavioural performance, trials were rank ordered from fastest to slowest RTs and placed into quintiles, allowing for comparison of the baseline and evoked-responses for the fastest and slowest $20 \%$ RTs (i.e. the optimum response and lapse domains). This technique has been used previously in the dual analysis of pupil and performance data in vigilance tasks (e.g. Murphy et al., 2011; Unsworth \& Robison, 2016; van den Brink et al., 2016).

\section{Data processing and statistical analysis}

Pupillometry data were preprocessed using the methods outlined in Chapter 3. Eyeblinks were reconstructed with linear interpolation and the data were smoothed with a 3rd-order Butterworth filter (4 Hz cut-off). The average amount of data replaced by blink interpolation across all participants that were included in the analysis was $18.3 \%$. Segments of pupil data $2500 \mathrm{~ms}$ in length were extracted for each trial, time-locked to the response (-1000 to $1500 \mathrm{~ms}$ ). These data were expressed as \%-modulation from a baseline calculated as the average pupil size in the $500 \mathrm{~ms}$ period prior to the RT-initiating stimulus event.

The data for each of the four outcome metrics were analysed separately using two-factor (Trial Group $\times$ Block) repeated measures ANOVA. Where appropriate, $p$-values were adjusted for violations of sphericity using the Greenhouse-Geisser correction. For the pupil analyses, trials were excluded if any portion of the prestimulus baseline had to be reconstructed with blink interpolation (20.79\% of trials). The pupil waveforms for the fastest and slowest $20 \%$ RTs were also subject to analysis with nonparametric permutation tests in order to assess significant modulation from baseline and the probability of significance for differences between the two groups. 


\section{Participant exclusions}

Two participants were excluded from the analysis for yielding poor quality pupil data (both had over 50\% interpolated data for baselines and over $80 \%$ interpolated data for evoked responses). The general pattern of results was the same both with and without the exclusion of these participants.

\subsubsection{Results}

\section{Performance data}

The distribution of RTs is shown in Figure 6.2. Average RT across all participants was $460 \mathrm{~ms}(S D=77)$. An ANOVA conducted on 1/RT (i.e. the reciprocal transform of RT: see Section 6.2.2) revealed a significant main effect of Trial Group, $F(4,88)=17.46, p$ $<.001, \eta_{p}^{2}=0.44$, a significant main effect of Block, $F(2,44)=28.29, p<.001, \eta_{p}^{2}$ $=0.56$, and a significant Trial Group $\times$ Block interaction, $F(8,176)=2.99, p=.004$, $\eta_{p}^{2}=0.12$. The nature of the interaction is illustrated in the top-left panel of Figure 6.3. Post hoc pairwise comparisons (Bonferroni-corrected) revealed that, in Trial Groups 1-3, 1/RT was significantly greater in Block 1 compared to Blocks 2 and 3 (all $p s<.05$ ). Additionally, 1/RT in Trial Group 4 was significantly greater for Block 1 compared to Block $3(p<.05)$. No other comparisons were significant (all $p \mathrm{~s}>.05)$. Therefore performance, as indexed by 1/RT, was best overall in Block 1 compared to Block 2 and Block 3, although the magnitude of this effect decreased across Trial Groups.

The average number of lapses across all participants was $5.5(S D=3.22)$. An ANOVA conducted on the number of lapses revealed a significant main effect of Trial Group, $F(2.78,61.1)=2.84, p=.049, \eta_{p}^{2}=0.11$, and a significant main effect of Block, $F(2,44)=7.27, p=.002, \eta_{p}^{2}=0.25$. The Trial Group $\times$ Block interaction was not significant $(p>.05)$. Exploring the main effects, Bonferroni-corrected post hoc $t$-tests showed that there were significantly fewer lapses in Trial Group $2(M=0.26, S D=0.5)$ compared to Trial Group $5(M=0.54, S D=0.83: p<.05)$, and in Block $1(M=0.19$, $S D=0.42)$ compared to Block $3(M=0.57, S D=0.76: p<.05)$. No other pairwise comparisons were significant $(p>.05)$. Therefore, the number of lapses increased with time-on-task, both within and between blocks (illustrated in the top-right panel of Figure 6.3). Taken together, the 1/RT and lapse results are consistent with the prediction that performance would decline as time on-task increased. 

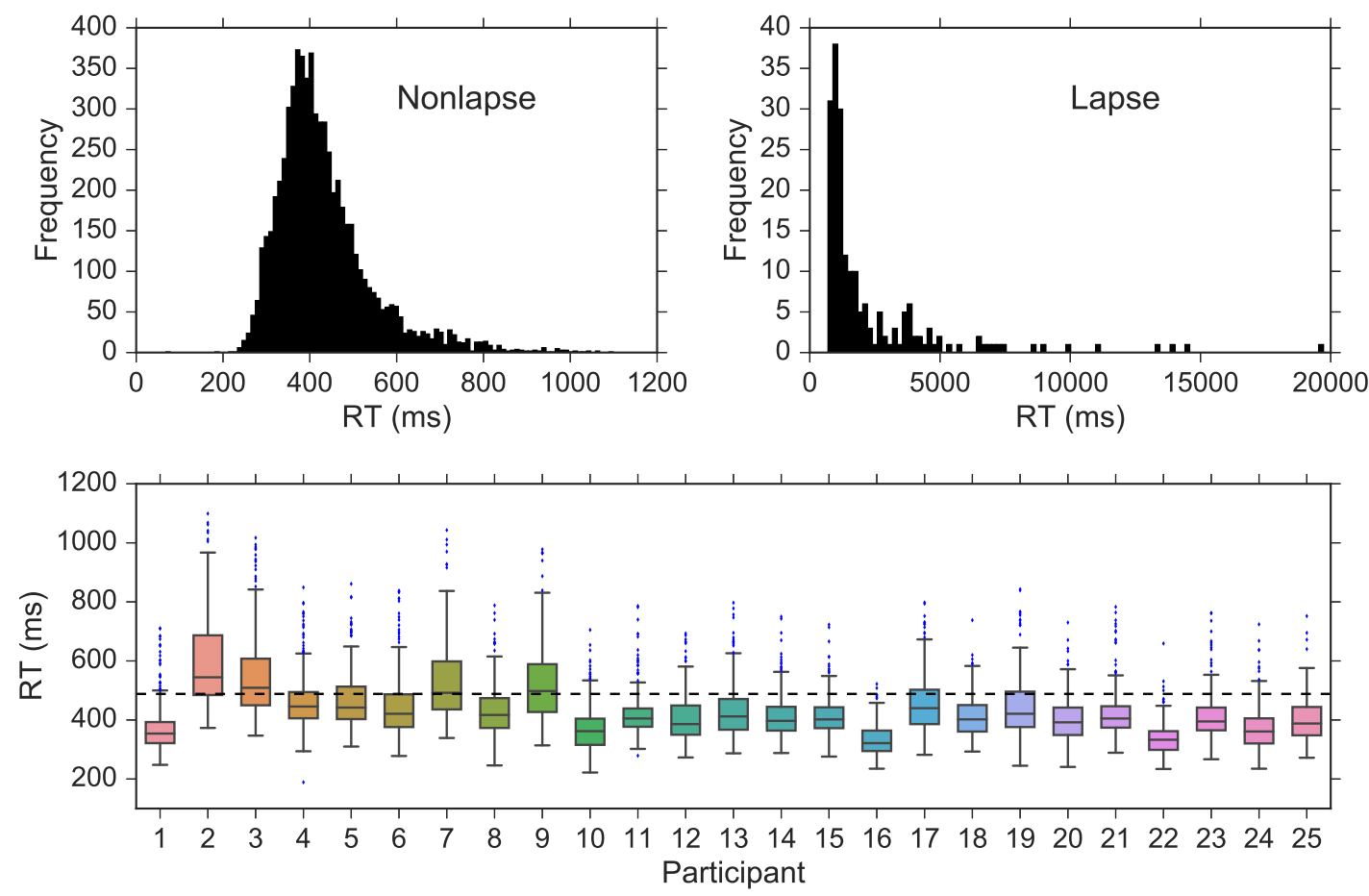

Figure 6.2. RT distributions in Experiment 5. The top-left and top-right panels show RT distributions across all participants for nonlapse and lapse trials, respectively. The bottom panel shows RT distributions for each individual participant, with the horizontal dashed line denoting the overall average RT. 


\section{Pupil data}

An ANOVA conducted on baseline pupil size revealed a significant main effect of Trial Group, $F(2.43,50.99)=13.12, p<.001, \eta_{p}^{2}=0.39$, a significant main effect of Block, $F(1.47,30.78)=5.75, p=.013, \eta_{p}^{2}=0.22$, and a significant Trial Group $\times$ Block interaction, $F(4.19,88.06)=4.64, p=.002, \eta_{p}^{2}=0.18$. The nature of the interaction is illustrated in the bottom-left panel of Figure 6.3. Exploring the interaction, Bonferroni-corrected post hoc $t$-tests revealed that baseline pupil size was significantly greater for Block 3 compared to Block 2 in Trial Group 2, and Block 3 compared to Block 1 in Trial Group 3 (all $p s<.05$ ). No other pairwise comparisons were significant $(p>.05)$.
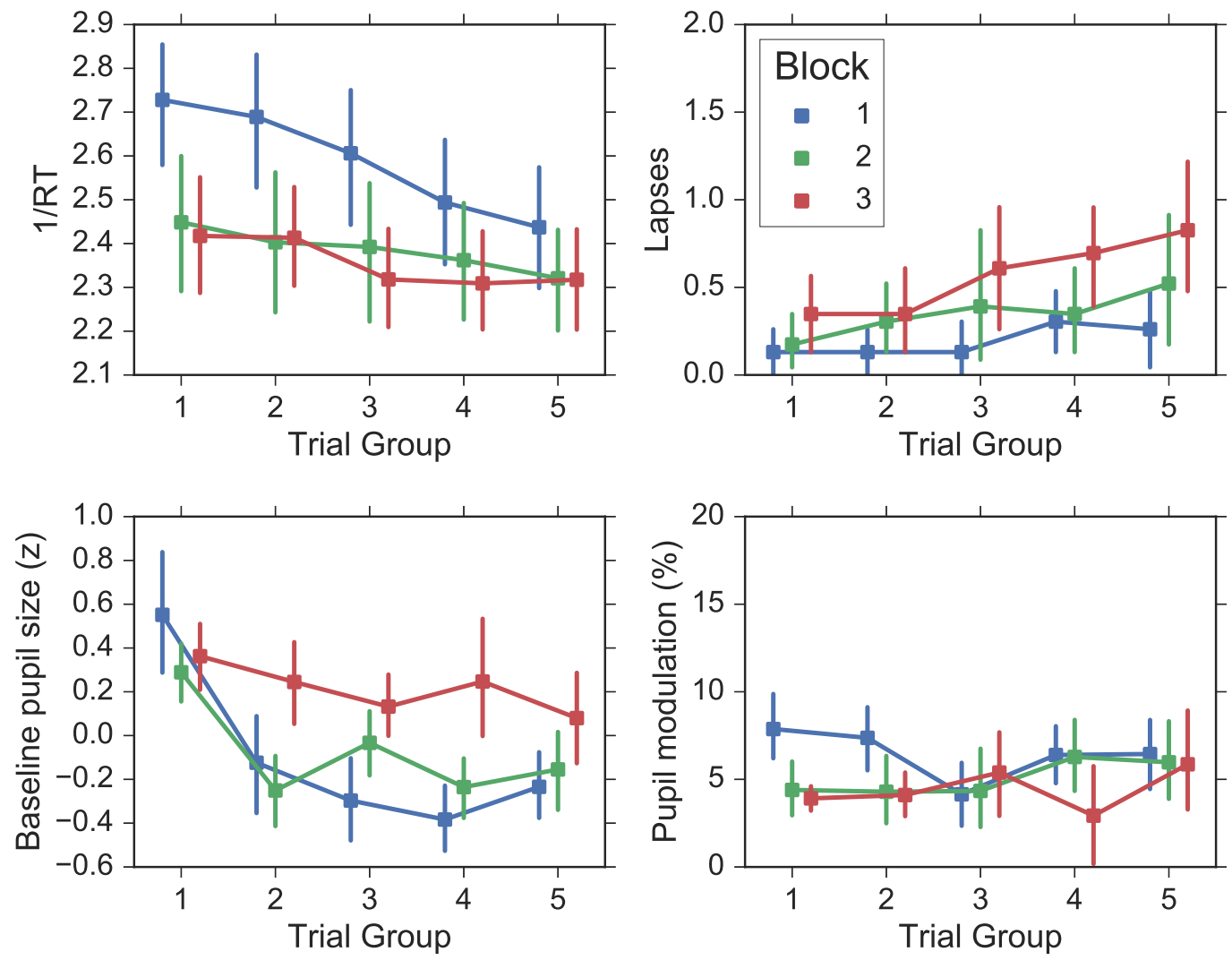

Figure 6.3. Performance (top row) and pupil (bottom row) measures across Trial Group and Block in Experiment 5, with error bars showing 95\% confidence intervals (bootstrapped, 1000 iterations).

Grand-average response-locked pupil traces for each Block are shown in Figure 6.4. The pupil began to dilate slowly following the stimulus event and then rapidly after the response. In the $1500 \mathrm{~ms}$ following the response, there was an average modula- 
tion of $5.22 \%$ and a peak latency of $880 \mathrm{~ms}$. An ANOVA conducted on the scalar pupil measures (as defined in Section 6.2.2) revealed no significant main effect of Trial Group on pupil modulation $(p>.05)$, but a significant main effect of Block, $F(2,42)=4.43, p$ $=.018, \eta_{p}^{2}=0.17$, and a significant Trial Group $\times$ Block interaction, $F(4.73,99.27)=$ $3.11, p=.014, \eta_{p}^{2}=0.13$. Bonferroni-corrected post hoc $t$-tests showed this effect to be driven by significantly greater pupil modulation for Block 1 compared to Block 2 and Block 3 at Trial Group 1, and Block 1 compared to Block 3 at Trial Group 2 (all ps $<.05)$. No other pairwise comparisons were significant $(p>.05)$. Overall, these data support the prediction that pupil size would decrease as time-on-task increased.

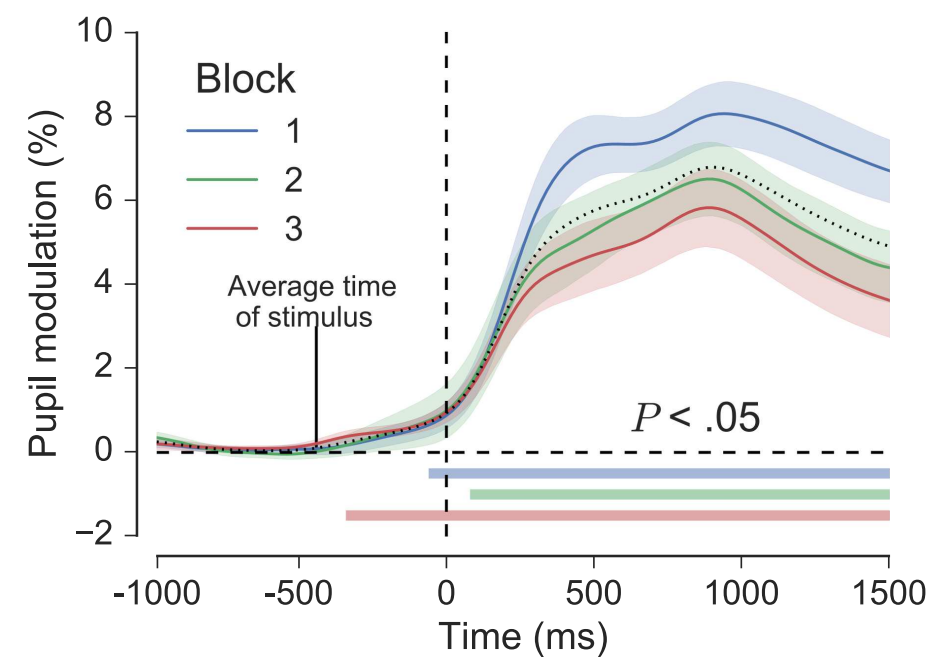

Figure 6.4. Average response-locked pupil traces for each Block in Experiment 5. The black dotted trace shows the overall mean. Shaded areas surrounding the coloured traces show the SEM (bootstrapped, 5000 iterations) and horizontal coloured bars denote clusters of significant modulation from baseline, as revealed by nonparametric permutation tests (1024 permutations, $p<.05$, cluster-corrected for multiple comparisons).

\section{Fastest vs. slowest responses}

The mean RT for the fastest $20 \%$ responses was $329 \mathrm{~ms}(S D=42 \mathrm{~ms})$, and for the slowest $20 \%$ it was $631 \mathrm{~ms}(S D=539 \mathrm{~ms})$. Figure 6.5 shows the pupillometry results for these two extreme quintiles. Baseline pupil size was larger on average for the fastest $20 \%$ of responses compared to the slowest $20 \%$, but the difference was not significant ( $p>.05$, left panel of Figure 6.5). The response-locked pupil traces for the fastest and slowest responses did differ significantly $(p<.05$, cluster-corrected permutation test, right panel of Figure 6.5). This difference pertained primarily to the timing and magnitude of the pupil response. For the slowest responses, the pupil began to dilate 
gradually around the time of stimulus onset (on average $631 \mathrm{~ms}$ prior to the response), and reached a peak of $8.24 \%$, whereas for the faster responses, dilation began after the button-press and reached a peak of 5.95\%. Both fastest and slowest responses shared a peak latency of around $860 \mathrm{~ms}$, but average modulation in the $2000 \mathrm{~ms}$ postresponse period was greater on average for the slowest $(M=7.05 \%, S D=1.13 \%)$ than for the fastest responses $(M=4.51 \%, S D=1.75 \%)$.
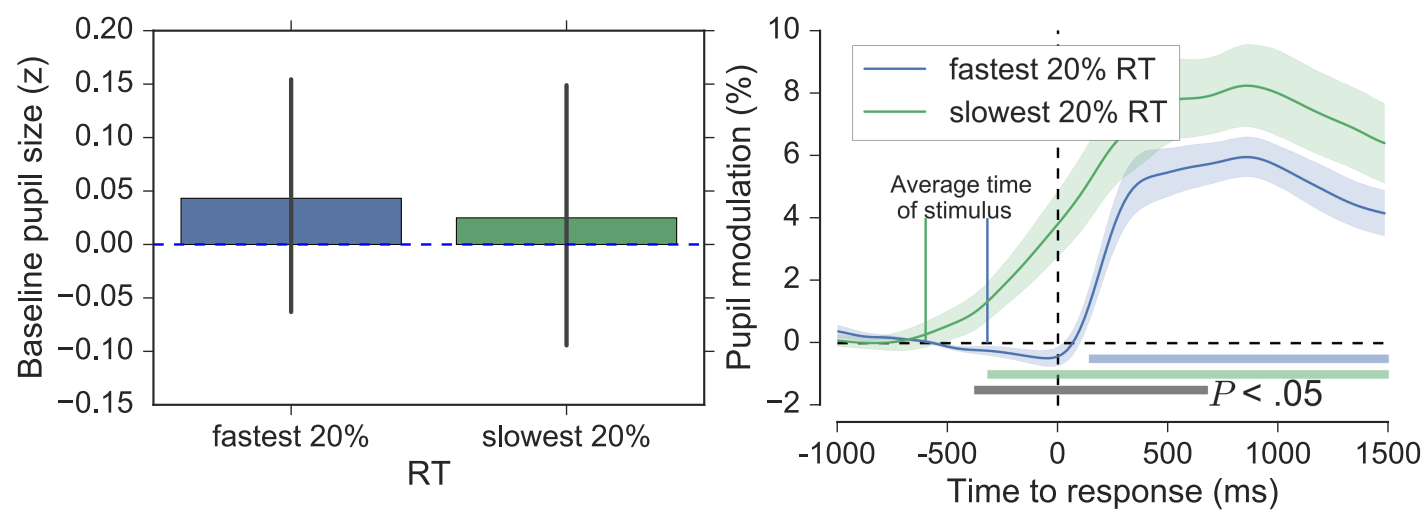

Figure 6.5. Baseline and response-locked pupil measures for the fastest and slowest $20 \%$ RTs in Experiment 5. The left panel shows mean prestimulus baseline pupil size with $95 \%$ confidence intervals (bootstrapped, 1000 iterations) and the right panel shows the evoked responses time-locked to response, with shaded areas surrounding the pupil traces denoting the SEM (bootstrapped, 5000 iterations). Horizontal coloured bars in the right-hand panel denote clusters of significant modulation from baseline for the respective traces (grey bar represents the difference between the traces), as revealed by nonparametric permutation tests (1024 permutations, $p<.05$, cluster-corrected for multiple comparisons).

\subsubsection{Discussion}

This experiment was conducted to further explore the relationship between pupil size and performance measures in a PVT. Participants monitored a low contrast gabor patch under the instruction to respond as quickly as possible every time the patch rotated $90^{\circ}$. The atypical stimulus approach was adopted to avoid confounds associated with the canonical running counter stimulus-namely its variable intensity and the performance feedback that it provides - which could potentially contribute to variance in behavioural and pupillometric measures (Thorne et al., 2005). Participants completed three successive blocks of the task taking only a 1 min break in between, and changes in performance and pupil measures were explored both within and between blocks. It was 
predicted that performance and pupil size would decrease as time-on-task increased, and that worse performance would be associated with smaller pupils at baseline.

The initial point to note is that the novel stimulus approach used in the current experiment lead to longer RTs than are typically observed in PVTs which use the canonical running counter stimulus. In PVTs using the running counter stimulus, average RT for subjectively alert participants is generally in the range of 200 to 300 ms (e.g. Basner et al., 2011; Blatter et al., 2006; Dorrian et al., 2007; Loh et al., 2004; Matsangas, Shattuck, \& Brown, 2016; McClelland et al., 2010; Wilkinson \& Houghton, 1982), whereas in the current PVT, also with subjectively alert participants, average RT was $460 \mathrm{~ms}$. This considerable difference can be readily attributed to differences in stimulus intensity. The running counter stimulus is dynamic and constantly changing, providing a constantly refreshed cue for the participant to respond, whereas a change in the orientation of a low-contrast gabor patch is more subtle and discrete, and issues no such constant cue to respond.

As predicted, the main performance measures analysed in the current experiment exhibited traditional time-on-task effects, with 1/RT decreasing and the number of lapses increasing as time-on-task increased. This general pattern was observed within and between each block of the PVT for both performance measures. For 1/RT, the biggest change was between the first block and the two subsequent blocks, with the difference being largest across the first three trial groups. Lapses were relatively few in number, but increased gradually and linearly within each block, and also between successive blocks. These patterns in the performance data were statistically robust even without the state manipulations (e.g. time of day, sleep deprivation) and large number of repeated tests that are often integral to the design of mainstream PVT research (e.g. Basner et al., 2011; Blatter et al., 2006; Dorrian et al., 2003; Graw et al., 2004; Loh et al., 2004; Manousakis, Maccora, Ftouni, \& Anderson, 2017).

With regards to the pupil data, the pattern of within-block declining baseline pupil size broadly reflected the decline in task performance, corroborating findings from previous PVT studies (Massar et al., 2016; Unsworth \& Robison, 2016) as well as various other studies which examined pupil and performance measures in tasks requiring vigilance (e.g. Grandchamp et al., 2014; Hopstaken, van der Linden, et al., 2015; McIntire, McKinley, \& Goodyear, 2014; van den Brink et al., 2016; Van Orden et al., 2000). However, the relationship between baseline pupil size and task performance was not simple and linear. Participants performed best and had the largest baseline pupil size at the beginning of each block, but the sharp drop in baseline pupil size which occurred between the first and second Trial Group did not accompany a commensurate 
drop in performance. Further, baseline pupil size was largest overall and showed the least variability across Trial Groups in Block 3, where performance was at its worst. In a similar fashion, the evoked responses were largest at the beginning of the first block, where performance was best, but were more erratic with respect to the performance data at other times. These patterns in the pupil data are consistent with the general prediction that pupil size would decrease as time-on-task increased, but they run contrary to the prediciton that worse performance would be reflected in smaller pupils at baseline.

Previous experiments have provided conflicting evidence as to whether optimal task performance is associated with larger or smaller pupils at baseline (e.g. Kristjansson et al., 2009; Unsworth \& Robison, 2016, see also Section 6.1.4). To further explore this issue with the current data, baseline and evoked responses for the trials with the fastest and slowest 20\% RTs were compared. Whilst there was no significant difference in baseline pupil size between these two groups of trials, there was a clear difference between the observed pupil traces. For the faster RTs, dilation did not begin until after the response was made, whereas for the slower RTs, dilation began around the time of the stimulus, and increased gradually until after the response was made. Average modulation in the postresponse period was also greater for the slowest RTs. The finding of gradual dilation leading up to a button-response was observed in the previous two chapters, and is well documented in the pupillometry literature as an indication of the decision component of an overt response (e.g. Einhäuser et al., 2010; Richer \& Beatty, 1985). However, it is also known that genuine cognitive effects on pupil size tend not to develop until at least $220 \mathrm{~ms}$ from the causal event (Mathôt et al., 2018; Mathôt, Siebold, et al., 2015), so the finding of immediate yet gradual onset of dilation around the time of stimulus occurrence that was observed for slower RTs in the present study should be treated with caution. Likely this can be linked to the greater variability of RTs in the lapse domain.

The findings from the current experiment are generally consistent with previous studies showing time-on-task effects on performance and pupil size, but they do not align perfectly with a specific theory of vigilance. The understimulating and unrewarding nature of the task does however provide ripe conditions for mind-wandering, suggesting that mind-wandering may have been partly responsible for the decline in performance. Previous studies have also reported larger pupils at baseline during periods of mindwandering and poor task performance (e.g. Franklin et al., 2013; Smallwood et al., 2012; Smallwood et al., 2011; Unsworth \& Robison, 2016), which is the pattern that was observed in block 3 of the current experiment. Research also suggests that very short breaks can reduce mind-wandering and lead to performance improvements 
by temporarily boosting motivation (e.g. Ariga \& Lleras, 2011; Ralph, Onderwater, Thomson, \& Smilek, 2016; Ross, Russell, \& Helton, 2014), something which fits with the pattern of data in the current experiment, where participants' performance was restored to more-optimal levels after taking a 1-min break in between each block.

It is important to recognise that a number of factors relating to the individual state of the participants could have influenced the results of the present experiment. For example, performance in PVTs is well understood to be affected by sleep pressure (Blatter et al., 2006), time-of-day and its interaction with circadian rhythms (Graw et al., 2004; Van Dongen \& Dinges, 2005), and the consumption of stimulants such as caffeine (Van Dongen et al., 2001). The current experiment did not control for any of these factors, but this could easily be achieved in a subsequent study. For instance, circadian effects could be controlled for by excluding strong morning and evening 'types' (e.g. Horne et al., 1980) and testing participants at the same times during the day, after they have reported having similar amounts of sleep. Participants could also be instructed not to consume caffeine (or other stimulants) on the day of testing to eliminate this confound. Finally, rather than having participants complete only three blocks with a 1 min break in between, a more traditional PVT design involving multiple tests throughout the day could be used to explore how the relationship between pupil and performance measures changes over time.

One final point to note is that there were issues with the design of the current PVT which limit the extent to which it can be closely compared to other PVTs. The use of an alternative stimulus was desirable to avoid certain confounds, but the experiment also differed in terms of block length and ISI. In their general recommendations for the standardised design and analysis of PVTs, Basner and Dinges (2011) suggest using an ISI of 2-10 s and having a fixed block length of $10 \mathrm{~min}$. Due to the way the current experiment was programmed, ISI was 4-12 seconds and block length was variable ( $M$ $=12.8 \mathrm{~min}, S D=0.63 \mathrm{~min}$ ). In a future experiment, the design could be tweaked in order to bring it into closer alignment with the task specifications set out by Basner and Dinges (2011), which would broaden the basis for comparison of experimental findings in the wider literature.

Overall, the current experiment has provided a fresh perspective on the PVT and yielded results which have helped to characterise the relationship between pupil size measures and behavioural performance. 


\subsection{Experiment 6}

\subsubsection{Introduction}

The aim of Experiment 6 was to look in to the relationship between pupil and performance measures in a standard vigilance task with stimuli presented in the visual modality. Only one of the 'standard' vigilance experiments described in Section 6.1.2 used visual stimuli (McIntire, McKinley, \& Goodyear, 2014), but the analysis for this experiment was correlational in nature and simply explored how average pupil size and performance measures showed a similar decline across four successive 10-min periods of continuous performance. This is in contrast to the auditory vigilance experiments described in the same section (Beatty, 1982a; Gilzenrat et al., 2010; Murphy et al., 2011), where there was considerable focus on event-related responses. The present experiment therefore aimed to examine event-related pupil responses in a novel vigilance task with visual stimuli, whilst controlling appropriately for the PFE and luminance confounds.

A novel attentionally demanding vigilance task was designed which involved monitoring four centrally presented equiluminant visual stimuli for $30 \mathrm{~min}$ in order to detect and respond to brief targets occurring with temporal and spatial uncertainty against a high background event rate. A relatively high number of targets ( 6 per minute) were used in order to ensure that a suitable amount of event-related data would be generated for the analysis (e.g. Mackie, 1987). First, it was predicted that performance measures across successive 10 min blocks of task performance would show the classic vigilance decrement that has been reported widely in the literature. Second, based on the most consistent findings from the research outlined in Section 6.1, it was predicted that the magnitude of response-locked (i.e. 'phasic') pupil size changes would decrease across the duration of the task.

\subsubsection{Method}

\section{Participants}

Twenty-eight participants (23 females; age range $18-32$ years, $M=20.07, S D=2.8$ ) completed the experiment voluntarily or in exchange for course credit. All participants were students at Swansea University reporting normal or corrected-to-normal acuity and colour vision. The experimental protocol was approved by the Ministry of Defence Research Ethics Committee and the Department of Psychology Ethics Committee at Swansea University. Written informed consent was obtained from each participant. 


\section{Design}

The task used in the current experiment was designed to adhere to the general principles of standard vigilance paradigms (e.g. see Baddeley \& Colquhoun, 1969; Mackworth, 1948, 1950; Parasuraman \& Davies, 1976) whilst controlling for the PFE and stimulus confounds. In brief, participants were asked to monitor four low-contrast gabor patches arranged squarely around a central fixation circle. The gabor patches rotated synchronously in a clockwise ticking motion at a rate of 120 ticks per minute $\left(30^{\circ}\right.$ rotation per tick), and targets were defined as instances where one of the gabor patches became briefly out of sync with the others (i.e. it missed a tick: see Figure 6.1 for an illustration). The task lasted for $30 \mathrm{~min}$, during which time continuous monitoring was required. Six targets were presented every minute at pseudorandom intervals (there was a minimum of $6 \mathrm{sec}$ between each target and targets did not occur within $2 \mathrm{sec}$ of the beginning or end of the task), meaning the task contained 180 targets overall. Targets occurred equally often at all of the four locations, although this was randomised across the whole experiment, meaning that spatial uncertainty as to the location of the target would contribute to task difficulty (e.g. Broadbent, 1958; Mackie, 1987; Warm et al., 2008). All participants completed one trial of this experiment in a single testing session lasting approximately $40 \mathrm{~min}$.

\section{Stimuli and apparatus}

The stimuli in this experiment were four gabor patches enveloped within a cosine window (spatial frequency $=0.1, S D=12,39 \%$ contrast) arranged squarely around a central fixation circle, each spanning $1^{\circ} \times 1^{\circ}$ of visual angle (see Figure 6.6). The gabor patches were generated online at https://www.cogsci.nl/gabor-generator and saved in JPEG format. The vigilance task was presented on a 24-in. Ilyama monitor running at a resolution of $1024 \times 768$ with a refresh rate of $144 \mathrm{~Hz}$ and responses were collected on a standard computer keyboard. The monitor and eye tracker were enclosed such that the only direct illumination came from the display screen. The screen was viewed from a distance of $40 \mathrm{~cm}$, which was maintained by a chin rest and forehead bar. Using a colorimeter (ColorCAL MKII, Cambridge Research Systems), the surface luminance of the grey background was recorded as $73.54 \mathrm{~cd} / \mathrm{m}^{2}$, and the surrounding dark light of the unused portion of screen as $0.53 \mathrm{~cd} / \mathrm{m}^{2}$. Pupil size and gaze data were recorded monocularly (left eye) using an EyeLink 1000 (SR Research, Mississauga, Ontario, Canada) system in tower mount configuration sampling at 1000 Hz. Eye level and camera position remained constant throughout the recording session 
for each participant. Stimulus presentation was managed with Experiment Builder (SR Research, Mississauga, Ontario, Canada).

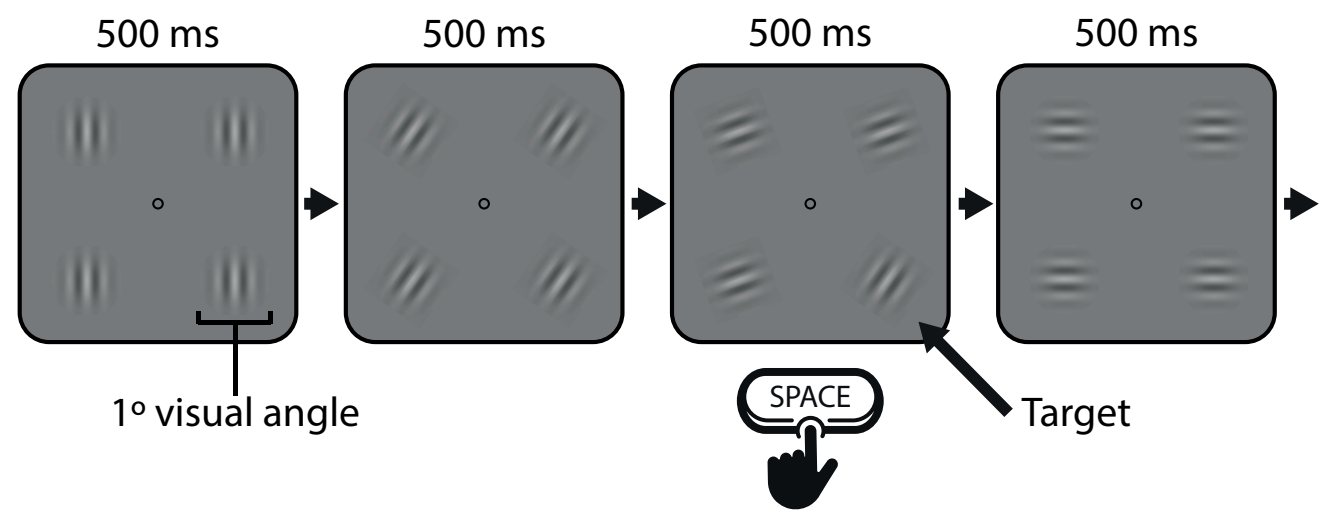

Figure 6.6. Schematic diagram showing the stimuli and trial sequence of Experiment 6. Participants had to respond by pressing space every time one of the gabor patches did not follow the standard sequence, which happened 6 times every minute.

\section{Procedure}

On arrival to the lab, participants were told that for the next 30 min they would be required to monitor four circular patches rotating with a ticking motion at the centre of the screen. It was explained to them that, from time to time, one of the patches would briefly become out of phase with the others, and that this was the target to which they had to respond. Participants were not given any further information about the frequency or temporal and spatial uncertainty of the targets. Once participants were comfortable with the definition of a target, they were instructed that their task was to press the space bar as quickly as possible every time they noticed such an event. Participants were forewarned that the task was monotonous, but that they should try to be as accurate as possible. They were also instructed to maintain central fixation on the screen. A 5-point calibration and validation routine was performed at the start of the experiment.

\section{Performance and pupil measures}

Task performance was assessed with RT, accuracy (i.e. percent hits and false alarms), and the SDT measures sensitivity $\left(d^{\prime}\right)$ and response bias $(c)$. Hits, correct rejections, misses and false alarms were determined by using an iterative algorithm to assign button responses to stimulus events. For each response, the RT in milliseconds to the last target was calculated. If the RT was greater than $6 \mathrm{~s}$ (the minimum time between targets), the response was allocated to the nearest elapsed neutral event and counted as a false 
alarm. All remaining responses were then grouped together and a permissible range for hits was determined as \pm 3 MADs from the participant median RT. Accordingly, all responses which occurred within 119 to $1200 \mathrm{~ms}$ of targets were counted as hits, and responses with RTs outside this range, as previous, were allocated to the nearest neutral event and counted as false alarms. The resulting distribution of RTs and permissible hit range is illustrated in the top panel of Figure 6.7. Finally, targets without a valid response were counted as misses, and all remaining neutral events as correct rejections. This process of dealing with responses in sustained-attention tasks with high event rates is similar to that used by Esterman et al. (2016) and van den Brink et al. (2016). For the purposes of analysis, performance measures were calculated within 10-min periods of the task.

Scalar baseline and evoked pupil measures were derived for all stimulus events and behavioural responses. For stimulus events, baseline pupil size was defined as the average pupil size in the $500 \mathrm{~ms}$ prior to the event, whereas for behavioural responses the baseline period was offset by a further $500 \mathrm{~ms}$ (i.e. from -1000 to $-500 \mathrm{~ms}$ prior to the response) in order to avoid contamination from fluctuations in pupil size associated with response preparation (e.g. see Einhäuser et al., 2010; Richer \& Beatty, 1985). All baseline measures were $z$-scored across the whole experiment. Evoked responses were defined as the average \%-modulation in the $2000 \mathrm{~ms}$ period after the onset of stimulus events, and in the $1500 \mathrm{~ms}$ after the onset of key-press responses.

\section{Data processing and statistical analysis}

Pupillometry data were preprocessed using the methods outlined in Chapter 3. Eyeblinks were reconstructed with linear interpolation and the data were smoothed with a 3rd order Butterworth filter ( $4 \mathrm{~Hz}$ cut-off). The average amount of data replaced by blink interpolation across all participants that were included in the analysis was $10.7 \%$. Segments of pupil data $2500 \mathrm{~ms}$ in length were extracted for each stimulus and response event. This epoch included a $500 \mathrm{~ms}$ baseline, which for stimulus events was the 500 ms period prior to the onset of the event. For response events the baseline period was offset by a further $500 \mathrm{~ms}$.

To examine the general shape of pupil data associated with each of the signal detection theory outcomes, event- (misses and correct rejections) and response-locked (hits and false alarms) average pupil traces from across the whole experiment were compared with nonparametric permutation tests. For the effects of time-on-task, performance and scalar pupil measures were averaged within 10 min task periods and 
analysed with repeated measures ANOVAs. Where appropriate, $p$-values were adjusted for violations of sphericity using the Greenhouse-Geisser correction.

\section{Exclusions}

Pupil data associated with stimulus and response events were discarded if the participant blinked during the baseline period or if more than $50 \%$ of the data across the epoch of interest were interpolated. Overall, this lead to the discarding of pupil data for $20.57 \%$ of event-locked epochs and $14.64 \%$ of response-locked epochs. No participants were excluded from the analysis.

\subsubsection{Results}

\section{Task performance}

Overall, the average number of button responses made by participants during the task was $151(S D=51)$. The average percentage of hits (i.e. correct detections) and false alarms (i.e. errors of commission) were $63.43 \%(S D=15.12 \%)$ and $1.08 \%(S D=$ $1.46 \%$ ), respectively. The remaining performance data are summarised in Figures 6.7 and 6.8. The former shows the RT distributions for the whole experiment, including the permissible hit and false alarm ranges (top panel), as well as the individual participant distributions (bottom panel). The latter shows accuracy (percentage of hits and false alarms), RT, sensitivity $\left(d^{\prime}\right)$ and response bias (c), all as a function of Task Period. The average RT for all hits was $671 \mathrm{~ms}(S D=14 \mathrm{~ms})$. Average sensitivity and response bias across the whole experiment were $2.9(S D=0.68)$ and $1.08(S D=0.26)$, respectively, indicating that perceptual sensitivity to targets was good, but also that participants were generally biased to withhold responses to targets.

To examine the effects of time-on-task, one-factor (Task Period) repeated measures ANOVAs were conducted on each of the performance measures. First, the percentage of hits and false alarms were analysed. There was a significant main effect of Task Period on the percentage of hits, $F(2,54)=6.72, p<.002, \eta_{p}^{2}=0.2$, with Bonferroni-corrected $t$-tests showing that participants attained a significantly higher percentage of hits in Task Period $1(M=68.93 \%, S D=14.03 \%)$ compared to Task Period $2(M=61.7 \%, S D=16.82 \%)$ and Task Period $3(M=59.05 \%, S D=20.02 \%), p$ $<.05$. Task Period 2 and Task Period 3 did not differ significantly $(p>.05)$. There was no effect of Task Period on the percentage of false alarms, $(p>.05)$.

A one-way repeated measures ANOVA conducted on the RT data for hits revealed a significant main effect of Task Period, $F(2,54)=16.76, p<.001, \eta_{p}^{2}=0.38$. 
Bonferroni-corrected post hoc $t$-tests showed that RT was significantly lower in Task Period $1(M=643 \mathrm{~ms}, S D=76 \mathrm{~ms})$ compared to Task Period $2(M=676 \mathrm{~ms}, S D=76$ $\mathrm{ms})$ and Task Period $3(M=694 \mathrm{~ms}, S D=83 \mathrm{~ms}$ : both $p \mathrm{~s}<.05)$, but that there was no significant difference in RT between Task Period 2 and Task Period $3(p>.05)$. The same analysis was then conducted on the signal detection measures sensitivity $\left(d^{\prime}\right)$ and response bias $(c)$. There was no significant main effect of Task Period on sensitivity $(p>.05)$, suggesting that participants' ability to discriminate targets from nontargets did not change throughout the task. Task Period did however have a significant main effect on response bias, $F(1.62,47.76)=16.76, p=.003, \eta_{p}^{2}=0.38$. As with RT, Bonferroni-corrected post hoc $t$-tests showed that response bias in Task Period $1(M=$ $0.97, S D=0.26)$ was significantly lower than it was in Task Period $2(M=1.11, S D=$ $0.31)$ and Task Period $3(M=1.15, S D=0.33$ : both $p s<.05)$, but that there was no significant difference between Task Period 2 and Task Period $3(p>.05)$. This suggests that participants became more conservative as the task progressed, and were therefore more reluctant to report that a target was present. As predicted, these performance data are consistent with the classic vigilance decrement.

\section{Pupil data}

Pupil data were time-locked to button-responses for hits and false alarms, and to stimulus events for misses and correct rejections. This was to ensure the comparability of pupil data that were consistently affected by motor acts. The grand-average pupil traces for each of these behavioural outcomes are shown in Figure 6.9. Both response-locked outcomes (hits and false alarms) showed the usual pattern of pupil modulation associated with motor preparation and execution, with dilation beginning up to $500 \mathrm{~ms}$ before the response and peaking shortly afterwards. The permutation tests revealed significant modulation from baseline for hits and false alarms, and also a significant difference between the two outcomes (right panel of Figure 6.9). The main differences between the two traces can be summarised as follows. For hits, pupil modulation reached peak of $7.65 \%$ with a latency of $400 \mathrm{~ms}$ from the response, whereas false alarms reached a similar peak of $7.56 \%$, but with a latency of $640 \mathrm{~ms}$. Average modulation for hits was $3.52 \%$, whereas for false alarms it was $4.57 \%$. As regards the event-locked responses (misses and correct rejections), both outcomes resulted in significant modulation from baseline in the poststimulus period, and there was also a significant difference between the two outcomes (left panel of Figure 6.9). For correct rejections, there was an average modulation of $0.57 \%$ and a peak modulation of $1.19 \%$ at a latency of $2000 \mathrm{~ms}$; whereas 

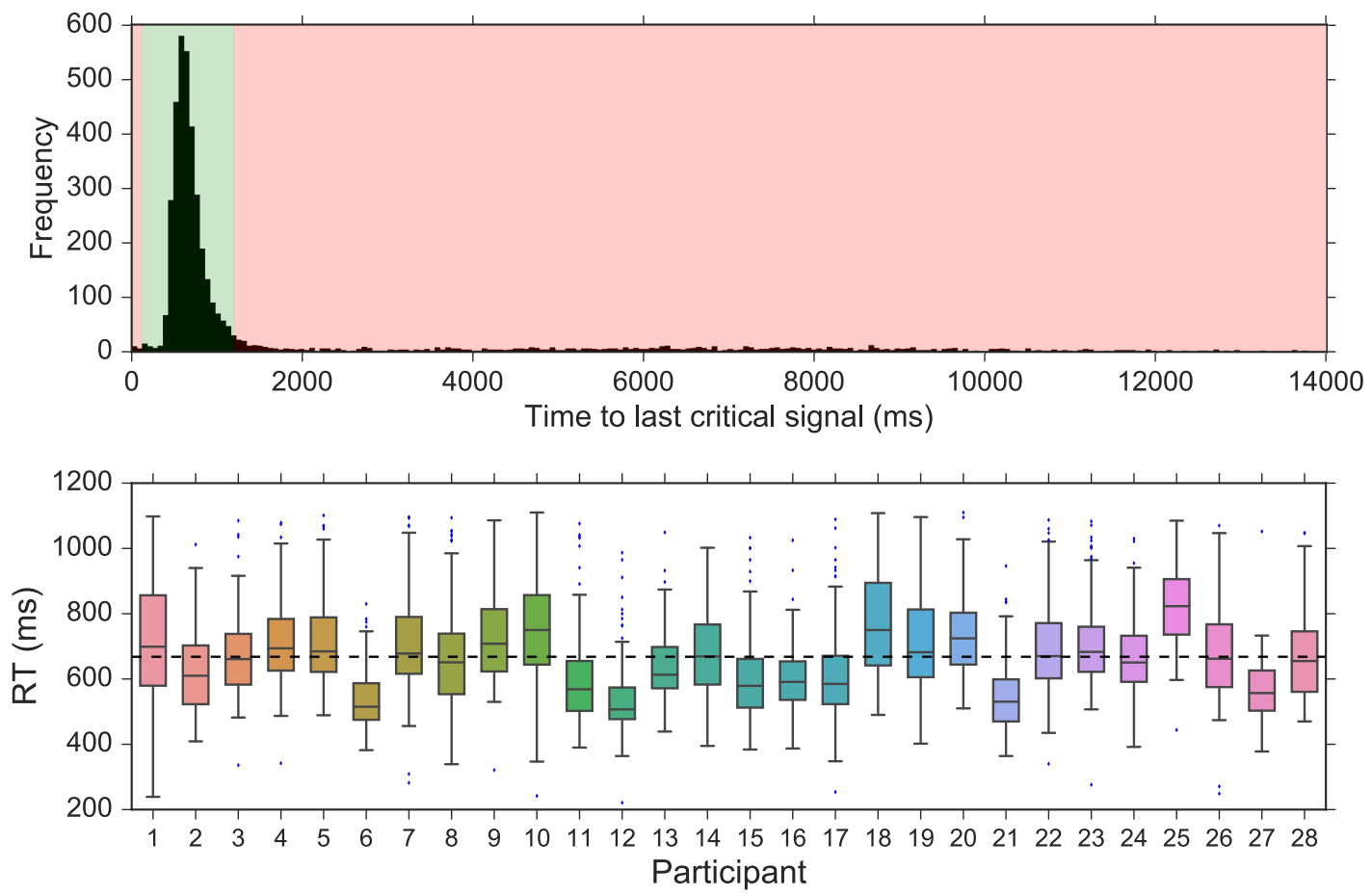

Figure 6.7. RT data from Experiment 6. The top panel shows the distribution of RTs across all participants in $50 \mathrm{~ms}$ bins. The green shaded area demarcates the permissible hit range (from 119 to $1200 \mathrm{~ms}$ after the target), and the red shaded area shows the range where responses were considered false alarms. The bottom panel shows the RT distribution for each individual participant (the horizontal dashed line denotes the overall average RT). 

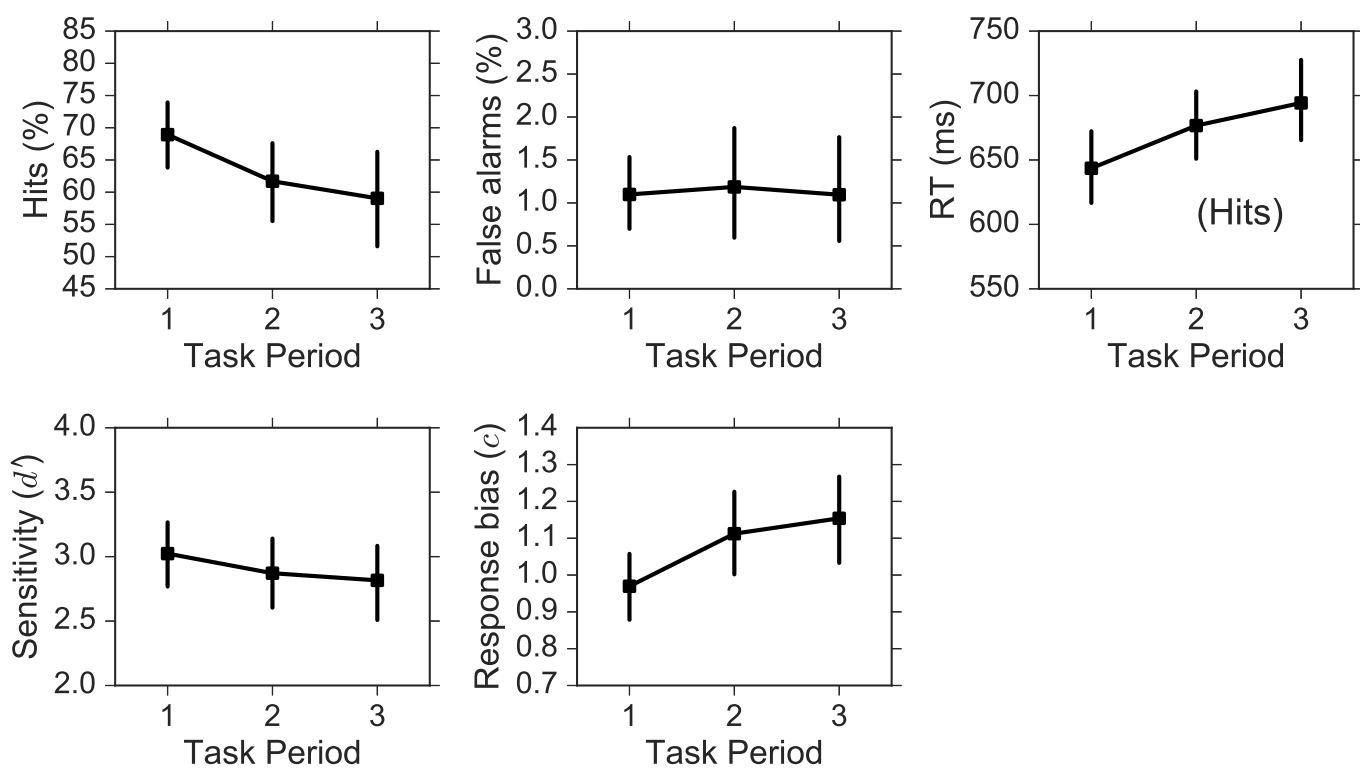

Figure 6.8. Performance measures for each Task Period in Experiment 6: the percentage of hits (top-left), RTs for hits (top-middle) and false alarms (top-right), sensitivity (bottom-left) and response bias (bottom-right). Error bars reflect 95\% confidence intervals (bootstrapped, 1000 iterations).

for misses, there was an average modulation of $1.9 \%$ and a peak modulation of $3.35 \%$ at a latency of $1500 \mathrm{~ms}$.

After examining the event- and response-locked pupil traces across the whole experiment, the scalar representations of pupil size (see Section 6.2.2) for all Outcomes were averaged across the three successive 10 min task periods and analysed with twoway (Outcome $\times$ Task Period) repeated measures ANOVAs. These data are displayed in Figure 6.10. For the event-locked responses, there was no significant effect of either Outcome or Task Period on baseline pupil size, and no significant Outcome $\times$ Task Period interaction (all $p \mathrm{~s}>.05$ ). For the evoked responses, there was a significant main effect of Outcome, $F(1,27)=30.13, p<.001, \eta_{p}^{2}=0.53$, with average \%-modulation being greater for misses $(M=1.84 \%, S D=2.61 \%)$ than for correct rejections $(M=$ $0.41 \%, S D=0.4 \%$ ). There was however no significant main effect of Task Period on the evoked responses, and no Outcome $\times$ Task Period interaction $(p<.05)$. For the response-locked analyses, there were no significant main effects of Outcome or Task Period on baseline pupil size, and no significant Outcome $\times$ Task Period interaction (all $p$ s $>$.05). With the evoked response-locked pupil data, there was no significant main effect of Outcome, but there was a significant main effect of Task Period $F(2,40)=$ 

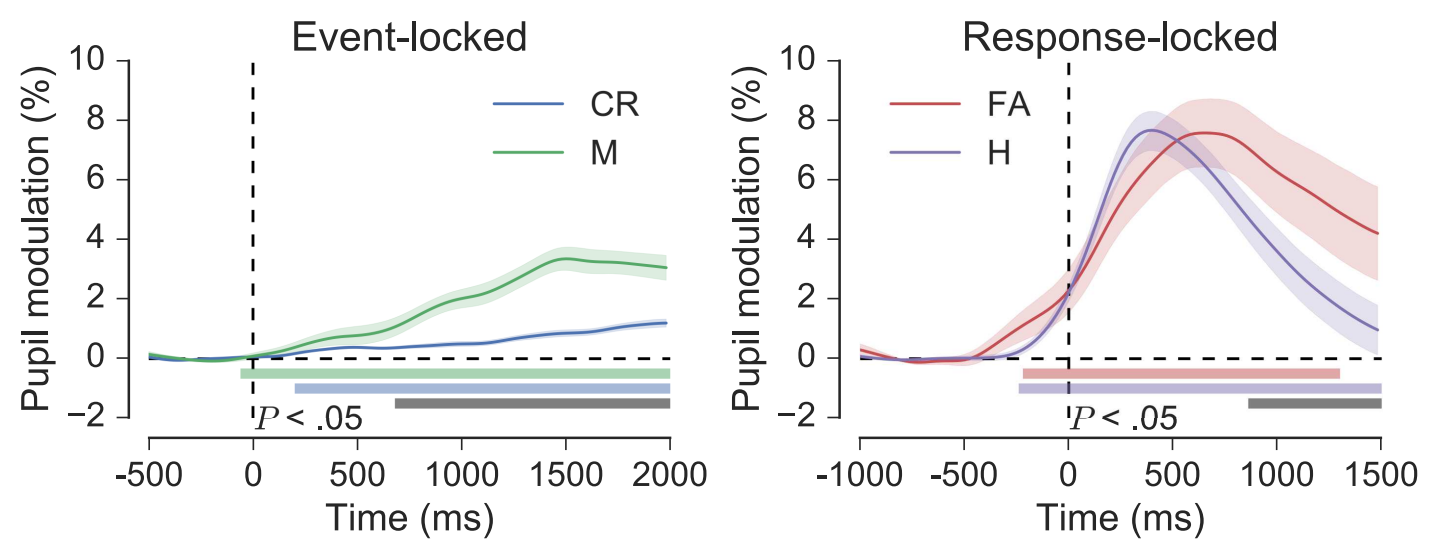

Figure 6.9. Grand average event- and response-locked pupil data in Experiment 6. The event-locked traces in the left panel show the average pupil response for correct rejections $(\mathrm{CR})$ and misses $(\mathrm{M})$, and the response-locked data in the right panel show the average pupil response for false alarms (FA) and hits $(\mathrm{H})$. The horizontal coloured bars indicate clusters of significant modulation from baseline and the gray bars show differences between the respective traces (1024 permutations, $p<.05$, cluster-corrected for multiple comparisons). Shaded areas surrounding the pupil traces denote the SEM (bootstrapped, 5000 iterations).

9.93, $p<.001, \eta_{p}^{2}=0.33$. Post hoc $t$-tests revealed that the average $\%$-modulation for Task Period $1(M=4.37 \%, S D=2.21 \%)$ was greater than it was for Task Period $2(M=$ $1.95 \%, S D=3.04 \%)$ or Task Period $3(M=1.23 \%, S D=2.43 \%$ : both $p$ s $<.05)$, but that Task Periods 2 and Task Period 3 did not differ $(p>.05)$. The Outcome $\times$ Task Period interaction was not significant $(p<.05)$. Overall, these patterns in the pupil data are consistent with the prediciton that the magnitude of phasic responses would decline as time-on-task increased.

\subsubsection{Discussion}

This experiment examined task performance and event-related pupil responses in a vigilance task with visual stimuli. Participants monitored four centrally located equiluminant stimuli continuously for $30 \mathrm{~min}$ with the instruction to respond by pressing the space bar every time they detected a target. The task required successive discrimination (see Footnote 2 of this chapter), but was made difficult by the brief target duration, high background event rate, and the temporal and spatial uncertainty as to the location of targets (Broadbent, 1958; Mackie, 1987; Warm et al., 2008). It was predicted that 
Event-locked pupil averages
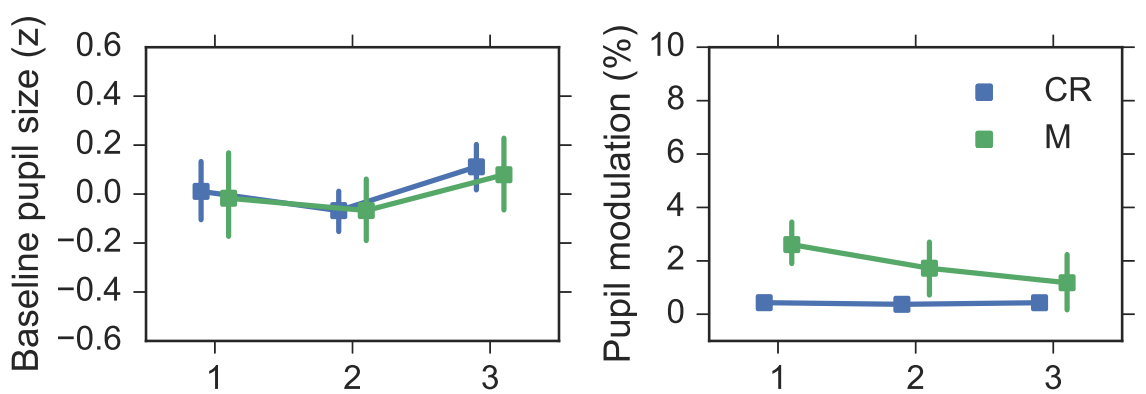

Response-locked pupil averages
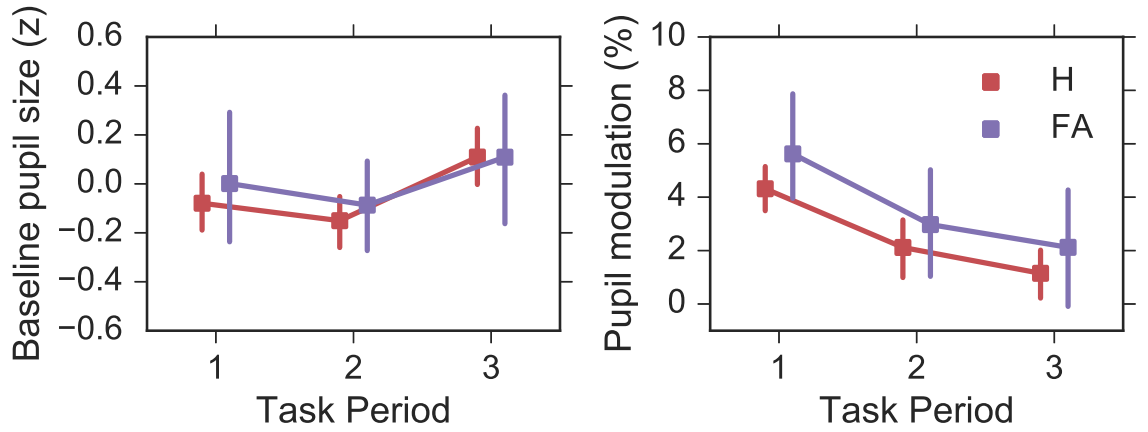

Figure 6.10. Event- (M: misses; CR: correct rejections) and response-locked (H: hits; FA: false alarms) pupil averages across each task Period in Experiment 6. Error bars reflect 95\% confidence intervals (bootstrapped, 1000 iterations). 
task performance and the magnitude of phasic pupillary responses would decrease as time-on-task increased.

Overall, the key performance measures were indicative of a classic vigilance decrement. Both the percentage of hits and the RT for hits changed across each successive 10 min period of the task in a manner reflecting declining performance. These findings are consistent with the well-established findings in the literature regarding the effects of time-on-task on detection performance under conditions of prolonged monitoring (e.g. Broadbent, 1953; Broadbent \& Gregory, 1965; Buck, 1966; Mackworth, 1948, 1950; Parasuraman \& Davies, 1976, 1982; Warm et al., 2008). The signal detection measures, sensitivity and response bias, were calculated to gain further insight into the cause of the declining percentage of hits. Sensitivity did not change across the task, but there was a conservative shift in response bias, suggesting that the decline in accuracy was linked to participants becoming less willing to report a detection, rather than a diminishing ability to discriminate targets from nontargets (Green \& Swets, 1974). This is consistent with previous reports that the vigilance decrement in tasks with high event rates is more closely related to changes in the strictness of the decision criterion over time, rather than perceptual sensitivity (e.g. Baddeley \& Colquhoun, 1969; Broadbent, 1971; Colquhoun, 1961; Parasuraman \& Davies, 1976).

Epochs of event-related pupil data were extracted for all signal detection outcomes in the current task in order to gain insight into the cognitive processing associated with these events. For misses and correct rejections, data were time-locked to the onset of the relevant stimulus event (left panel of Figure 6.9). Both misses and correct rejections resulted in gradual dilation of the pupil in the poststimulus period, suggesting that increasing pupil size was the dominant trend throughout the task (as correct rejections were by far the most numerous events). Notably, misses resulted in reliably greater pupil dilation than correct rejections, a similar observation to that which was made by Beatty (1982a) in an auditory vigilance experiment. As suggested by Beatty (1982a), from a signal detection perspective, the effect of an enhanced pupillometric response to missed targets may reflect increased processing of sensory information for stimuli which fall close to the decision criterion. However, in the present experiment, such an interpretation must be tempered against the possibility that the enhanced response following misses was linked to the preparatory effects of neighbouring button-presses which fell just outside of the permissible hit range. Previous research has shown that the pupillometric effects associated with motor responses can begin to emerge up to 1500 ms prior to the actual response (e.g. Einhäuser et al., 2010; Hupé et al., 2009; Richer \& Beatty, 1985), which means that false alarms with RTs between 1200 ms (the upper 
bound of the permissible hit range) and $2700 \mathrm{~ms}$ may have contributed to the dilatory effect observed for misses. For hits and false alarms, pupil data were time-locked to the button response and showed typical patterns of modulation associated with motor preparation and execution. The main difference between the pattern of dilation for hits and false alarms was that the pupil response for false alarms was larger and peaked later compared to hits. As suggested by Murphy et al. (2011), who made similar observations, this pattern in the data could be associated with the cognitive effects of a performance monitoring process. However, the larger pupil responses to false alarms may also be associated with the higher level of stimulus uncertainty which accompanies these events compared to correct detections (Yu \& Dayan, 2005).

To examine the effects of time-on-task on pupil dynamics, scalar values of baseline and evoked pupil size were calculated for all stimulus and response events and then averaged within thirds of the task (Figure 6.10). Baseline pupil size for all outcomes was relatively unchanged across the duration of the task, a finding which is consistent with the results from the auditory vigilance experiment conducted by Beatty (1982a). This suggests that the mode of organismic activation linked to fluctuations in tonic pupil diameter was not related to the central processes which underpinned the performance decrement observed in the present task. In contrast to this however, the task-evoked pupil responses for misses, hits and false alarms exhibited a marked decline in magnitude across each successive task period. This pattern of change closely paralleled the decline in performance indexed by the percentage of hits and RT for hits, and is therefore consistent with the findings from the vigilance experiments of Beatty (1982a) and Murphy et al. (2011), as well as various other pupillometric studies of tasks requiring sustained attention (e.g. Hopstaken, van der Linden, et al., 2015; Unsworth \& Robison, 2016).

Task-evoked pupillary responses have been linked to phasic activation of the LC-NA system by a broad range of neurophysiological and behavioural studies in both human and non-human primates (e.g. Alnaes et al., 2014; Aston-Jones \& Cohen, 2005; Beatty, 1982a, 1982b; de Gee et al., 2017; Einhäuser et al., 2008; Gilzenrat et al., 2010; Hou et al., 2005; Jepma \& Nieuwenhuis, 2011; Joshi et al., 2016; Murphy et al., 2014; Murphy et al., 2011; Phillips et al., 2000; Rajkowski et al., 1993; Urai et al., 2017; Varazzani et al., 2015, see also section 2.5.3 of this thesis). Further to this, single-unit recording studies in animals have found that phasic activation of the LC-NA system occurs typically in response to task-related events during periods of high performance (e.g. Aston-Jones, Chiang, \& Alexinsky, 1991; Aston-Jones, Rajkowski, Kubiak, \& Alexinsky, 1994; Rajkowski, Kubiak, \& Aston-Jones, 1994). For example, the study by 
Aston-Jones et al. (1994) revealed that noradrenergic neurones in the monkey LC are phasically activated by infrequent target cues during a vigilance task, and also that the amplitude of these phasic responses diminishes over time. On the basis of the tight link between task-evoked pupil responses and phasic activity in the LC, such findings from animal studies bear a striking resemblance to those from the present study.

It is difficult to ascertain whether the findings of the current experiment fit best with a resource depletion, mind wandering, or resource control-failure account of the vigilance decrement (see Caggiano \& Parasuraman, 2004; Smallwood \& Schooler, 2006; Thomson et al., 2015, note also section 2.2.3). Key parts of the puzzle in this regard are whether participants experienced the task as being effortful and the extent to which they engaged in task-unrelated thought throughout, but this information was not obtained in the current experiment. The reason for not obtaining such information is that it requires the use of intermittent thought probes (e.g. Hopstaken, van der Linden, et al., 2015; Smallwood et al., 2004; Unsworth \& Robison, 2016), which involve temporary disengagement and therefore undermine a key aspect of vigilance task design: the requirement for continuous monitoring (Parasuraman \& Davies, 1976). Thought probes may also serve as 'mini breaks', which can disrupt task monotony and therefore improve the vigilance decrement (Ariga \& Lleras, 2011; Ralph et al., 2016; Ross et al., 2014). In the absence of subjective reports, only the pupil data and the nature of the task can serve as a basis for inferring the cause of the vigilance decrement. First, the task itself was prolonged and monotonous, and participants were without an incentive to maintain high levels of performance - conditions which provide fertile grounds for a wandering mind (Smallwood \& Schooler, 2006). Second, the magnitude of task-evoked pupillary responses reduced across the course of the task, something which has previously been associated with disengagement (Hopstaken, van der Linden, et al., 2015) and mindwandering (Smallwood et al., 2011). Therefore, taken together, this suggests that the vigilance decrement in the current experiment may have been linked primarily to mindwandering or resource control-failure, but further data would be required to confirm this.

In terms of its design, the current task can be criticised for its lack of resemblance to real-world vigilance tasks - a criticism that is often directed at laboratory-based studies of vigilance (e.g. Hancock, 2013; Mackie, 1987; Wiener, 1987). The two most notable aspects of task design which invite such criticism are the unrealistically short duration of the vigil and high target rate. Real-world vigilance tasks which take place in operational settings can last for many hours of uninterrupted time, and targets may be as rare as 1 per week (Wiener, 1987). These conditions are clearly much different 
from those of the present task, where the vigil lasted for only 30 minutes and targets were presented at a rate of 6 per minute. As Wiener (1987) points out, however, for laboratory-based studies, short vigils and high target densities are practically necessary in order to generate enough data for analysis, and there is likely not much to be gained from conducting lengthy low target density studies of vigilance.

In sum, the present study replicated the well-known vigilance decrement-the reduction in detection performance that takes place during conditions of prolonged and continuous monitoring. Evoked pupil responses declined across the duration of the task whereas baseline pupil size was mostly unchanged, suggesting that the performance decrement may have been linked to gradual disengagement of attention as the task progressed, rather than a change in organismic arousal state. However, this interpretation is not conclusive due to the lack of self-report data, which is the only means of determining the extent to which participants engaged in task-unrelated thought and experienced the task as being difficult. It is also unclear how much the lack of incentive contributed to poor task performance. Therefore, although the current study provided further insight into the relationship between pupil size and detection performance, the precise cause of the vigilance decrement could not be ascertained. A future implementation of these methods may benefit from collecting self-report data once participants have completed the task and exploring the effects of different reward conditions on both performance and pupil data.

\subsection{Overall discussion}

Recent pupillometric studies of vigilance and sustained attention have suggested that measurements of pupil size could potentially be used in operational settings to monitor performance, and to perhaps even predict and prevent errors associated with lapses of attention before they occur. However, as was made clear in the introductory sections of this chapter, the literature in this area-especially with regards to tasks using visual stimuli-is relatively sparse, and differences in methodology and task requirements have lead to conflicting findings. The purpose of the current chapter was therefore to further explore the relationship between pupil size and performance measures within the context of well-established task frameworks from the vigilance literature.

Perhaps the most consistent finding across both experiments regarding the relationship between pupil size and monitoring performance was that, in line with previous experimental findings (e.g. Beatty, 1982a; Hopstaken, van der Linden, et al., 2015; Unsworth \& Robison, 2016) and the predictions of established theory (Aston-Jones \& 
Cohen, 2005), task-evoked (or 'phasic') pupil responses were generally largest when performance was at its best. This trend was most consistent in Experiment 6, where the decline in detection performance was almost perfectly mirrored by a decline in the magnitude of task-evoked responses associated with hits, misses, and false alarms. In Experiment 5, the relationship between task-evoked responses and performance measures was less consistent, although the largest responses did occur when performance was highest (i.e. at the beginning of the first block). In general, these findings suggest that changes in task-evoked pupil responses may serve as an accurate indication of general task engagement, with a decline in their magnitude over time reflecting cognitive disengagement from the task and an increased likelihood of suboptimal performance.

Less consistent overall in terms of its relationship with performance was baseline, or 'tonic', pupil size. In Experiment 5, baseline pupil size decreased on average both within and between blocks, and although this was the general trend with performance, the relationship between the two was erratic. Moreover, in Experiment 5, baseline pupil size was mostly unchanged across three successive blocks of the task, despite a marked decrement in performance. This lack of consistency fits well with the conflicting findings from the literature summarised in the introductory sections of this chapter, and generally suggests that the relationship between baseline pupil size and task performance is complex, and in need of further characterisation. One possibility raised by van den Brink et al. (2016) is that the effects of time-on-task on baseline pupil size obscure a more nuanced relationship with performance. This suggestion was based on evidence from a gradual-onset performance task (described in Section 6.1.3), where regressing out the effects of time-on-task from the baseline pupil data revealed a quadratic relationship with performance, such that performance was optimal when baseline pupil size was at intermediate levels.

Overall, the experimental work presented in this chapter supports the general notion that changes in pupil size can be used to gain insight into monitoring performance in long and demanding tasks. However, there is clearly a need for further research in order to determine the practical feasibility of utilising pupil size as a psychophysiological marker of attentional lapses in real-time monitoring systems for use in operational settings. Characterising the precise relationship between different measures of behavioural performance, task-related factors and patterns of pupil behaviour will be a crucial next step in this regard. 


\section{Chapter 7}

\section{General discussion}

\subsection{Introduction}

The central aims of this thesis were to explore how eye tracking and pupillometry can be used to gain insight into the cognitive processes involved in visual search and vigilance tasks and to examine the potential for using these methods to assist with socially important tasks in operational settings. Six experiments were designed specifically to build on recent findings suggesting that fluctuations in pupil size under conditions of constant luminance are a useful index of cognitive processes relating to effort and target detection in visual search (Klingner, 2010a; Porter et al., 2007; Privitera et al., 2010), and attentional state and detection performance in vigilance tasks (Kristjansson et al., 2009; Unsworth \& Robison, 2016; van den Brink et al., 2016). The purpose of the present chapter is to discuss the key findings of these experiments within the broader context of the methodological and theoretical themes of the thesis, and to consider their wider implications for practical applications in operational settings. This section summarises the key points from the introductory and experimental chapters and then narrows the focus for the remaining discussion.

\subsubsection{Summary of introductory chapters}

In Chapter 1, the methods of eye tracking and pupillometry were introduced within the context of cognitive psychology, brain-computer interface (BCI) technology, and the field of human-computer interaction (HCI). The research and practical applications of these methods were outlined briefly, and their flexibility and unobtrusiveness were identified as key practical advantages over other physiological measurement techniques such as EEG and fMRI. Recent advances in the performance, availability and affordability of 
eye tracking hardware, together with recent findings in the domains of visual search and vigilance, were recognised as solid grounds for further exploring the practical potential of these methods. The central aim of using the methods to further scientific understanding of the cognitive processes involved in visual search and vigilance tasks, both in laboratory and in applied settings, was established.

Chapter 2 was a detailed literature review of six key topics: attention, working memory, vigilance, visual search, eye tracking and pupillometry. Attention and working memory were introduced as the fundamental cognitive processes responsible for allowing us to selectively process specific sources of information and to keep this information in an accessible state over time. Emphasis was placed on the visual modality of perception. The topics of vigilance and visual search were then defined and reviewed individually. In both cases, key methods, findings and theories from over 70 years of psychological research literature were reviewed. Emphasis was placed on findings that have emerged from research in laboratory settings, but factors pertaining to visual search and vigilance in the real-world were also considered. Finally, the methods of eye-tracking and pupillometry were described in terms of their research and practical applications, with a specific focus on the use of these methods for investigating vigilance and visual search. The section on pupillometry contained an advanced review of the research literature on cognitive and neuroscientific points of interest, as well as practical applications.

Chapter 3 began by detailing some of the key methodological principles of infrared video-based eye tracking and then focused on general aspects of data collection and processing specific to the EyeLink 1000 system used in the current thesis. Pupillometry methods were then described. The technical issues of stimulus confounds and the pupil foreshortening error (PFE) were raised due to the complications they pose for cognitive pupillometry. Data processing and statistical analysis techniques used for pupil data throughout the thesis were then detailed. The chapter concluded with a brief summary of the thesis up to that point and an overview of the coming experimental chapters.

\subsubsection{Summary of experimental chapters}

The first experimental chapter of this thesis was Chapter 4. The experiments described in this chapter were designed to explore the effects of effort and target detection on pupil size during visual search, whilst circumventing the issue of the PFE. Manipulations of set size and target presence were implemented in both experiments and compact search arrays were presented briefly close to the point of fixation, with participants 
being instructed not to move their eyes during experimental trials. In Experiment 1, large pupil dilations were observed in the poststimulus period but they showed little variability with respect to the experimental manipulations, despite performance data suggesting differences in cognitive processing between conditions. Stimulus contrast (black 'C's appearing on a white background) and response preparation and execution were identified as substantial contributors to the observed dilations. Experiment 2 attempted to eliminate these components from the pupil responses by altering the stimuli and task design. Participants instead searched for low-contrast tilted gabor patches and responded only when cued to do so after a delay period. Small dilations were observed in the poststimulus period even with the removal of stimulus and motor confounds, but still there was small variability with respect to set size and target presence. The pupil responses to search stimuli in this experiment were attributed to generalised cognitive processes associated with task engagement and the forced-choice nature of the task. Taken together the results of Experiments 1 and 2 emphasised the importance of controlling for stimulus and task-related confounds, highlighted the additive nature of cognitive effects on the pupil response, and revealed the general limitations of using brief stimulus presentations in pupillometric studies of visual search.

Chapter 5 continued the exploration into the cognitive pupil dynamics of visual search but with the alternative approach of allowing eye movements and attempting to control and correct for the PFE. Experiment 3 replicated an established data-driven procedure for quantifying and correcting the PFE. Participants tracked a target as it traced a circular pattern around the screen and pupil data were estimated as a function of $x$ and $y$ gaze coordinates using multiple linear regression. All regression equations and predictor variables were significant, and coefficients were subsequently used to noticeably reduce gaze position artefacts in the pupil data. Experiment 4 - the main experiment in this chapter-was a multiple target search task where participants reported the number of targets $(0,1,2$ or 3$)$ via mouse click after completing the search. Wholetrial analysis of pupil data revealed large dilations at search offset for 3-target trials, a finding which was linked to the increased proportion of self-terminations with a keypress in this condition. Fixation-aligned pupillary response averaging revealed small transient dilations following target but not distractor fixation. This effect, which did not depend on a motor response, was robust at the group level but less consistent at the participant level, and even less so at the level of individual fixation epochs. Using the coefficients derived from Experiment 3 it was possible to correct the pupil data in Experiment 4 for gaze position artefacts, but this had little impact on statistical outcomes. Overall, Experiments 3 and 4 demonstrated that confounds associated with 
visual stimuli and the PFE can be overcome within the context of free-viewing visual search to effectively isolate search-related processes.

The final experimental chapter-Chapter 6-examined the relationship between pupil and performance measures during visual vigilance task performance. Experiment 5 looked at pupil and performance measures in a psychomotor vigilance task (PVT). The task implemented a novel stimulus approach in order to minimise the effects of luminance and contrast on the pupil response, as well as other confounds associated with the canonical 'running counter' stimulus approach used for PVTs, such as performance feedback and variable stimulus intensity. Performance decrements, as measured by changes in RT and the number of lapses, were apparent both within and between successive blocks of the task. Baseline pupil size declined with time-on-task in a manner that loosely mirrored the decline in performance. Task-evoked pupil responses were greatest at the beginning of the experiment, where performance was at its best, but their relationship with performance throughout the remainder of the experiment was inconsistent. Experiment 6 examined pupil and performance measures in a 'standard' vigilance task, which demanded continuous monitoring for $30 \mathrm{~min}$ on the part of the participant in order to detect and respond to relatively infrequent target stimuli occurring against a background of neutral events. A classic vigilance decrement in detection performance and RT was observed across the duration of the task. Baseline pupil size was mostly unchanged, but there was a marked reduction in the amplitude of task-evoked responses associated with misses, hits and false alarms which mirrored the decline in performance. Mind-wandering was proposed as a likely source of the vigilance decrement, but tentatively so, due to the lack of self-report data on taskexperience and thought content. In general, Experiments 5 and 6 supported the notion of a relationship between pupil size measures and detection performance in vigilance, but they confirmed that this relationship is neither simple nor consistent.

\subsubsection{Current focus}

The remainder of this chapter will focus on discussing the key pupillometry findings from the experimental work of this thesis within the broader context of the methodological and theoretical themes that were developed in the introductory chapters. First, methodological issues in cognitive pupillometry are discussed and recommendations made on the basis of current experimental outcomes, knowledge from the wider research literature and general practical considerations. The experimental findings of the thesis are then discussed within the theoretical contextxs of vigilance, visual search, 
and the LC-NA system. Questions for future research are then outlined and practical implications considered before concluding with some final remarks.

\subsection{Methodological issues and recommendations}

Cognitive pupillometry, the main research technique used in this thesis, involves making inferences about mental processes on the basis of pupil size changes which occur in response to events associated with experimental manipulations. Changes in pupil size arising from cognitive effects are however diminutive in nature, with dilations typically being on the order of tenths of a millimetre in size (Beatty \& Lucero-Wagoner, 2000). This makes them difficult to dissociate from the noncognitive effects on pupil size, such as the true effects associated with stimulus properties and the pupil's reflexes, and the apparent effects caused by eye movements and optical distortion in video-based eye tracking hardware (i.e. the PFE). Controlling adequately for these confounds was a central theme in the experimental methods of this thesis. This section therefore revisits the issues of stimulus confounds and the PFE, and provides recommendations for dealing with them on the basis of current experimental outcomes and the wider research literature. The general implications of these issues as regards the scope for effective use of cognitive pupillometry methods in laboratory and applied settings are discussed.

\subsubsection{Stimulus confounds}

As noted in Section 3.3.1, for experiments with visual stimuli, an important prerequisite for cognitive interpretations of pupil size changes is confidence that they were caused by the psychological and not the physical characteristics of the stimuli. Reviewing the early pupillometry literature, Goldwater (1972, p. 344) expressed concerns that these two effects may be 'hopelessly confounded', and questioned the overall validity of studies which had used visual stimuli, such as the sexual interest studies of Hess and Polt (1960) and Hess et al. (1965). Goldwater was right to draw attention to these issues, as the stimuli of the studies in question were not balanced for luminance, colour, contrast or spatial frequency, all of which are known to affect pupil size to varying degrees (Barbur et al., 1992; Barbur et al., 1998; Kohn \& Clynes, 1969; Loewenfeld, 1966; Ukai, 1985; Watson \& Yellott, 2012; Woodhouse, 1975). However, Goldwater's assertion that these confounds can not be effectively controlled carries less authority today than it did then. 
Many of the early studies in cognitive pupillometry (e.g. Bradshaw, 1968; Colman, 1969; Hess \& Polt, 1960, 1964; Hess et al., 1965; Kahneman \& Beatty, 1966, 1967; Paivio \& Simpson, 1966; Simpson \& Paivio, 1966) used techniques which, though innovative, lacked accuracy and precision due to low sampling rates-typically 1-2 photographic pictures of the eye per second-and hand measurements of a printed or projected image of a pupil with a millimetre ruler. The methods of stimulus preparation and presentation that were available at the time also limited the extent to which control could be exerted over the physical properties of the stimuli. Today, the situation is quite different, as advances in eye tracking hardware have increased the frequency, accuracy and precision with which pupil size measurements can be obtained. For instance, the EyeLink 1000 system used for data collection in the current thesis can sample at up to $1000 \mathrm{~Hz}$ and resolve pupil size to within 0.01 of a millimetre (SR Research Ltd., 2010). In addition to advances in eye tracking hardware, developments in computerised methods of stimulus preparation and presentation have brought researchers increasing control over their stimuli, allowing for careful balancing across a range of physical dimensions and millisecond accurate timing of stimulus exposure. As a consequence of these advances, the last 20 years in particular have seen a substantial increase in the number of cognitive pupillometry experiments effectively employing visual stimuli (e.g. Aboyoun \& Dabbs, 1998; Alnaes et al., 2014; Binda, Pereverzeva, \& Murray, 2013b, 2014; Bradley et al., 2008; Cavanagh et al., 2014; de Gee et al., 2017; de Gee et al., 2014; Hopstaken, van der Linden, et al., 2015; Hupé et al., 2009; Jessen, AltvaterMackensen, \& Grossmann, 2016; Kafkas \& Montaldi, 2011; Klingner, 2010a; Klingner, Tversky, \& Hanrahan, 2011; Kloosterman et al., 2015; Kristjansson et al., 2009; Laeng \& Endestad, 2012; Laeng \& Falkenberg, 2007; Laeng, Ørbo, Holmlund, \& Miozzo, 2011; Laeng et al., 2013; Laeng \& Sulutvedt, 2014; Leknes et al., 2013; Marshall, 2002; Mathôt, Siebold, et al., 2015; Naber, Alvarez, \& Nakayama, 2013; Naber et al., 2011; Naber \& Nakayama, 2013; Nuske, Vivanti, Hudry, \& Dissanayake, 2014; Porter, Leonards, et al., 2010; Porter, Tales, et al., 2010; Porter et al., 2007; Preuschoff et al., 2011; Privitera et al., 2014; Privitera et al., 2010; Smallwood et al., 2011; Unsworth \& Robison, 2017; Urai et al., 2017; van den Brink et al., 2016; van der Meer et al., 2003; Wierda et al., 2012; Wolff, Scholz, Akyürek, \& van Rijn, 2015).

On the whole, the experiments in the current thesis can be added to this growing body of literature as further evidence that visual stimulus confounds can be adequately controlled for in the laboratory in order to reveal cognitive effects on pupil size. There was an issue in Experiment 1 where the use of high-contrast stimuli (black ' $C$ 's on a white background) was identified as being partly responsible for the large stimulus- 
evoked pupil dilations, but following the remedial action of using low-contrast gabor patches of various orientation as stimuli in Experiment 2, the magnitude of the stimulusevoked dilations was dramatically reduced. Therefore, what was originally identified as a methodological shortcoming actually served as an instructive demonstration of how control over stimulus confounds is absolutely necessary and can make a substantial difference to experimental outcomes.

One obvious point to note is that the stimuli used in the current thesis were kept very basic - either Landolt 'C's (Experiments 1 and 4) or gabor patches (Experiment 2, 5 and 6) - and targets differed from nontargets only in terms of orientation, rather than some other stimulus dimension which would be more likely to confound the pupil responses such as colour, brightness or size. It is often the case however that a research question may call for the use of more complex visual stimuli. For instance, researchers have used pupillometry to investigate cognitive processing in the perception of faces (e.g. Jessen et al., 2016; Laeng et al., 2013; Leknes et al., 2013; Nuske et al., 2014), objects (Alnaes et al., 2014; Kafkas \& Montaldi, 2011) and complex scenes (Mathôt, Siebold, et al., 2015; Naber, Frässle, et al., 2013; Naber \& Nakayama, 2013; van den Brink et al., 2016). In such cases, it is recommended to use monochrome stimuli and, if possible, to use stimulus matching software (e.g. the SHINE Toolbox for MATLAB: Willenbockel et al., 2010) to achieve precise control over the effects of low-level image properties such as luminance, contrast and spatial frequency. In circumstances where stimulus appearance would inevitably involve a change in overall display luminance then it is advisable to use masking techniques (e.g. Klingner, 2010a; Klingner et al., 2011; Mathôt et al., 2013; Montefinese, Ambrosini, Fairfield, \& Mammarella, 2013; Porter et al., 2007) to prepare the pupil for the impending luminance change. Such a technique was implemented in Experiment 4 of the current thesis, where the individual display items of the visual search arrays were masked with stimuli of near-identical luminance prior to their appearance.

A final point to consider before closing the issue of stimulus confounds is the extent to which they constrain the potential for using pupillometry to achieve practical aims in applied settings. In the laboratory it is possible to exert strict control over both the ambient testing conditions and the physical properties of the stimuli to which participants are exposed. In applied settings however, such control is usually unattainable. For example, when undertaking visual search and monitoring assignments like airport baggage screening and long distance driving, system operators are necessarily exposed to a range of complex and variable stimuli in dynamic environments. For a practical application of pupillometry to be useful in such scenarios, the pupillometric effect upon 
which it is based (or the analysis techniques that are used to resolve that effect) must be robust to the effects arising from changes in retinal illumination as the operator scans her visual environment. This inescapable requirement strongly suggests that, as far as stimulus confounds are concerned, the future of applied cognitive pupillometry will depend on the development and refinement of sophisticated analysis techniques for separating cognitive and luminance effects (e.g. Marshall, 2002; Pomplun, Sunkara, Fairley, \& Xiao, 2011), combined use with other physiological measures to produce composite indices of mental processes (e.g. Backs \& Walrath, 1992; Libby et al., 1973; Marshall, Pleydell-Pearce, \& Dickson, 2003), simplification of operational tasks and environments, or combinations thereof.

\subsubsection{The pupil foreshortening error}

The PFE is inherent to video-based eye tracking systems. It occurs when eye rotation causes the shape of the pupil, as it appears from the fixed position of the camera, to become squashed and distorted, which in turn causes the eye tracker's recorded value of pupil size to decrease despite there being no actual change. Crucially, the artefactual pupil size changes arising from the PFE can be much larger than true changes that are brought about by psychological manipulations. Given that the majority of commonly used eye tracking systems (e.g. the EyeLink 1000, SR Research, Ottawa) can not distinguish between real and artefactual pupil size changes, the PFE issue is problematic and has important implications for both the design of pupillometry experiments and the analysis and interpretation of pupil data.

The most simple and straight-forward method of circumventing the PFE is to design experiments where participants do not move their eyes during trials. This approach is recommended by hardware manufacturers (e.g. SR Research Ltd., 2010, p. 110 ) and has been used widely in the pupillometry research literature (e.g. Hopstaken, van der Linden, et al., 2015; Otero et al., 2011; Privitera et al., 2010; Smallwood et al., 2011; Wierda et al., 2012), but it is a significant design constraint for tasks such as reading ${ }^{1}$ and visual search, where eye movements play an important role. Therefore, an alternative approach in tasks involving eye movements is to correct for the PFE after the data have been collected (e.g. Brisson et al., 2013; Gagl et al., 2011; Hayes \&

\footnotetext{
${ }^{1}$ In the case of reading, tasks which only require visual word recognition can use the aforementioned approach of presenting words sequentially at the same position on the screen (e.g. Heaver \& Hutton, 2011; Montefinese et al., 2013; Otero et al., 2011; Võ et al., 2008). In theory this method could also be used to investigate sentence processing (e.g. see Franklin et al., 2013), but not without the loss of ecological validity and the ability to obtain many of the common measures that are used to examine reading performance, such as saccade length, fixation duration and regressions to previous words.
} 
Petrov, 2015), or render it unsystematic through the careful design of tasks and stimuli (e.g. randomising or counterbalancing stimulus positions: see Cavanagh et al., 2014; Klingner, 2010a, for examples).

This thesis explored each of the aforementioned methodological approaches to countering the PFE. In the visual search experiments of Chapter 4, compact stimulus arrays were presented for brief durations around the point of fixation and trials were excluded from the analysis if participants made substantial eye movements. This eliminated the PFE confound, but it also imposed practical constraints on the experimental manipulation of set size, which was intended to probe the pupil's response to search difficulty. Given that the search component of the tasks in Experiments 1 and 2 was conducted on stimulus representations held in VWM (which has severe capacity limitations: note Section 2.1.2), it was not practical to use the larger set size differences such as had previously been reported to reveal pupillometric effects of effort in visual search (e.g. Porter et al., 2007). This constraint ultimately resulted in a lack of variability in the pupil data with respect to set size. Chapter 5 explored the alternative approach of allowing eye movements during search and countering the PFE through the random positioning of display elements and with a data-driven correction procedure. Together with complementary techniques for analysing the pupil data, this approach revealed robust cognitive effects of pupil dilation associated with target vs. distractor fixation and with the preparation and execution of a nonmandatory motor response for terminating search. Interestingly, the significance of the group-level effect of target vs. distractor fixation in Experiment 4 was not contingent on data-driven correction for the PFE. Overall, this suggests that the PFE may be adequately countered in free-viewing visual search paradigms simply by preparing stimuli in such a way as to render eye movement strategies (mostly) random. It should be noted however that this approach may not be feasible in other free-viewing tasks, such as natural scene viewing, where the locations of task-relevant stimuli can not be subject to rigorous control. In these circumstances, corrective measures should be applied. It may also be possible to discourage idiosyncratic viewing strategies by varying the location of gaze position at the start of each trial.

In terms of real-world applications, countering the PFE with such methods as have been applied in this thesis presents a more-complex challenge, as operational settings provide less scope for control over task and stimuli, and idiosyncratic eye movement strategies, which are often essential to the task in hand, have the potential to exacerbate the error. This means that post-hoc correction of pupil data for gaze position artefacts is not sufficient, and that the error would have to be accounted for in 
real-time. The ideal solution in such cases would be a video-based eye tracker with built-in correction for the PFE. In theory, eye trackers which work with a physical model of the eye (e.g. Pupil Labs, Berlin, Germany), could achieve this using such model-based correction techniques as were described in Section 5.1.1 (Mathôt et al., 2018). On the other hand, eye trackers which work with the pupil / corneal reflection tracking principle (described in Section 3.2.1) are generally limited to the basic measure of arbitrary pixel units (i.e. the number of pixels subtended by the pupil image on the camera sensor), which is strongly influenced by eye rotation. As suggested by Klingner (2010b, p. 99), eye trackers which first measure pupil size as the length (in pixels) of the major axis of an ellipse fitted to the pupil image and then convert this to millimetres using a scaling factor derived from (for example) interpupillary distance (e.g. the Tobii 1750, Tobii, Stockholm, Sweden) may be less sensitive to the PFE. However, such methods are still prone to errors caused by inhomogeneities in the thickness of the iridic musculature (Jay, 1962), the nonsymmetrical nature of pupil dilation (Wyatt, 1995), and various other hardware related factors, such as fluctuations in the amount of infrared illumination that reaches the eye.

\subsubsection{Other considerations}

Beyond stimulus confounds and the PFE, there are other methodological considerations relating to age and disease which have bearing on the practical utility of cognitive pupillometry in both research and applied settings. Ageing has been linked to reduced pupil size in general, diminished amplitude and velocity of the dark reflex, and prolonged recovery time of the PLR (Bitsios, Prettyman, \& Szabadi, 1996). Further to this, research comparing groups of younger and older adults suggests that the usefulness of pupillary responses as indicators of cognitive processes diminishes with age (e.g. Piquado, Isaacowitz, \& Wingfield, 2010; van der Meer et al., 2003). Similar effects to those associated with age have also been reported when comparing the pupillary responses of patients who suffer from Alzheimer's disease to those of healthy agematched controls (e.g. Porter, Leonards, et al., 2010; Prettyman, Bitsios, \& Szabadi, 1997). These findings are problematic from a methodological perspective, as they suggest an additional age- and disease-related constraint on the practical potential of cognitive pupillometry. A further complication in the case of Alzheimer's disease is that its tau neuropathology is known to originate in the $\mathrm{LC}$ - the activation of which is closely associated with the cognitive pupillary response-many years before diagnosis 
(Mather \& Harley, 2016) ${ }^{2}$. This means that individuals who are in the very early stages of tau pathology may exhibit abnormal pupillary responses which could confound experimental research or confuse applied systems. The reverse corollary of this however is the intriguing possibility that pupillometry, either alone or in combination with other measures, could be utilised to index early sings of tau pathology, and thereby open the gateway to early treatment and preventative measures before the disease progresses.

\subsection{Theoretical context of experimental findings}

Even when methodological issues are appropriately addressed, the utility of pupillometry as a tool for gaining insight into cognitive function during any form of visual task hinges on what is currently known about the neural underpinnings of psychosensory reflex dilation. As explained in Section 2.5.3, a large body of behavioural and neuroscientific research in human and nonhuman animals implicates the activity of the LC-NA system in the cognitive modulation of pupil size. Most notably, microsimulation and neural recording studies in monkey (Joshi et al., 2016; Rajkowski et al., 1993; Varazzani et al., 2015) have demonstrated a tight temporal coupling between nonluminance mediated pupil-size changes and the moment-to-moment activity of the LC, suggesting that the true value of pupillometry as a research tool is in its ability to serve as a proxy for the activity of this subcortical brain structure. Therefore, to understand what the experimental findings of this thesis say in terms of human cognition and information processing during visual search and vigilance, it is necessary to consider them within the broader context of current theory regarding the functional role of the LC-NA neuromodulatory system. This part of the discussion summarises prominent theories of LC-NA function (cited in Section 2.5.3) and then considers how they fit with the pupillometric effects observed in the visual search and vigilance experiments of this thesis.

\subsubsection{Theories of $\mathrm{LC}$-NA function}

Neurons of the brainstem nucleus LC are the sole source of NA, a neuromodulator that is widely understood to play a central role in arousal, autonomic function and cognition (Berridge, 2008; Berridge et al., 2012; Berridge \& Waterhouse, 2003; Mather \& Harley, 2016; Samuels \& Szabadi, 2008a, 2008b; Sara, 2009; Sara \& Bouret, 2012, see also Sections 2.5.3 and 2.2.4). Early theories regarding the function of the LC-NA

\footnotetext{
${ }^{2}$ With a sample of 61 brains from ages 21-30, Braak, Thal, Ghebremedhin, and Tredici (2011) reported that $59(96.7 \%)$ of them showed some sign of tau pathology in the LC.
} 
system focused on its role in the regulation of vigilance and sleep-wake cycles (e.g. Hobson, McCarley, \& Wyzinski, 1975; Roussel, Buguet, Bobillier, \& Jouvet, 1967), but the discovery of the LC's afferent noradrenergic projections throughout the cortex (Cedarbaum \& Aghajanian, 1978) gave rise to further interest in putative links with other important cognitive functions such as attention, memory formation and retrieval, learning, decision making and sensory processing (e.g. Mason \& Iversen, 1978; Neuman \& Harley, 1983; Sara, 1985a, 1985b; Sara \& Segal, 1991). Mounting evidence from electrophysiological recording and pharmacological studies in behaving rodents and primates eventually lead to the proposal of three specific theories of LC-NA function all within a very close time-frame: adaptive gain and optimal performance (Aston-Jones \& Cohen, 2005), network reset (Bouret \& Sara, 2005) and unexpected uncertainty (Yu \& Dayan, 2005). The theories are summarised presently.

\section{Adaptive gain and optimal performance}

One of the most notable findings to emerge from neural recording studies with behaving primates is that there are two distinct modes of LC output: a tonic mode, characterised by elevated baseline activity and increased behavioural distractibility, and a phasic mode, characterised by short bursts of activity coupled tightly with accurate behavioural responses during task performance (Aston-Jones, Rajkowski, \& Kubiak, 1997; AstonJones et al., 1994; Clayton, Rajkowski, Cohen, \& Aston-Jones, 2004; Rajkowski et al., 2004; Usher, Cohen, Servan-Schreiber, Rajkowski, \& Aston-Jones, 1999). In the study by Usher et al. (1999), for example, where primates were trained to press a lever in response to frequent visual targets but to withhold responses to infrequent distractors, high phasic activity and low tonic activity were associated with correct responses to targets and distractors, respectively, the reverse being true for incorrect responses. Such findings prompted Aston-Jones, Rajkowski, and Cohen (1999) to suggest that these two modes of output may have different cognitive roles, specifically that phasic activity may promote selective or focused attention and that the tonic mode may bring about a state of behavioural flexibility and attentiveness to the wider environment. Growing evidence in support of these ideas eventually lead Aston-Jones and Cohen (2005) to formulate their adaptive gain theory, which proposes that the function of the LC-NA system is to optimise the trade-off between exploitative and explorative behaviour. Specifically, phasic bursts of NA in response to relevant environmental stimuli facilitate behavioural responses to the outcome of task-specific decision processes, whereas increases in tonic activity in response to low task utility heighten the organisms awareness to the possibility of more valuable opportunities. These effects are thought to be mediated 
by the action of NA in adjusting the gain (i.e. responsivity) of cortical circuits that are involved in the performance of specific tasks.

\section{Network reset}

Research into crustacean nervous systems has demonstrated that the synchronous input from a small number of neuromodulatory neurons can suddenly interrupt activity in neural networks and facilitate their dynamic reconfiguration into new functional networks which support adaptive changes in behaviour (e.g. Meyrand, Simmers, \& Moulins, 1994; Simmers, Meyrand, \& Moulins, 1995). Analogously, in vivo electrophysiological studies with primates and rats have shown that changes in LC activity correspond closely with changes in the behavioural contexts within which they are measured (e.g. Aston-Jones et al., 1997; Bouret \& Sara, 2004; Rajkowski et al., 2004; Vankov, HervéMinvielle, \& Sara, 1995). For instance, Aston-Jones et al. (1997), who trained primates to release a lever rapidly for a juice reward in response to infrequent visual target stimuli, observed strong phasic LC responses selective to the target. However, when the stimulus-reinforcement contingency was abruptly reversed such that the reward was delivered following correct responses to a new target, the LC neurons quickly stopped responding to the old target and began responding to the new one, a change which occurred many trials in advance of any overt behavioural adaptation. Inspired by such findings in invertebrates and mammals, Bouret and Sara (2005) proposed their 'network reset' theory of LC-NA function. The central idea behind this theory is that NA release in response to environmental imperatives serves as a general reset signal which facilitates the reconfiguration of forebrain networks that are involved in specific cognitive functions, thereby promoting rapid behavioural adaptation (Bouret \& Sara, 2005).

\section{Unexpected uncertainty}

Yu and Dayan (2005) proposed a role for NA in signalling unexpected uncertainty, specifically that which is elicited by environmental changes which evoke sensory impressions that are robustly at odds with top-down expectations. This is in contrast to expected uncertainty, which is born out of the known unreliableness of predictive relationships within a familiar environment, and is instead proposed to be signalled by the neuromodulator acetylcholine (ACh). According to Yu and Dayan (2005), NA and ACh work together to signal these different forms of uncertainty and thereby enable optimal learning and inference in noisy and changeful environments. The theory is formalised within a complex Baysian framework and takes support primarily from 
pharmacological, physiological and behavioural data linking NA and ACh to specific aspects of a broad spectrum of cognitive processes (e.g. Gil, Connors, \& Amitai, 1997; Hasselmo, 1999; Hsieh, Cruikshank, \& Metherate, 2000; Kimura, 1999; Kobayashi et al., 2000; Posner \& Petersen, 1990).

\subsubsection{Experimental findings in context}

The above theories and the evidence upon which they are based all agree that behavioural context plays an important role in determining LC output. It therefore follows naturally that cognitive pupil responses, which are thought to be concomitant with underlying activity in the LC-NA system, should be interpreted in relation to the behavioural context within which they were observed. This includes the general nature of the task, its stimuli and parameters, the specific instructions given to the participant, and any incentives that may be involved. With the above in mind, the present section takes an overarching look at the general pattern of pupil responses observed in the visual search and vigilance experiments of this thesis and considers what they may indicate in terms of cognition and information processing.

\section{Button pressing}

In all visual search and vigilance experiments, button-presses were associated with significant pupil dilations. This effect pervades the cognitive pupillometry literature and has been observed in many different forms of task (e.g. de Gee et al., 2014; Einhäuser et al., 2010; Einhäuser et al., 2008; Fahle et al., 2011; Hupé et al., 2009; Kloosterman et al., 2015; Porter et al., 2007; Privitera et al., 2014; Privitera et al., 2010; Richer et al., 1983; Smallwood et al., 2011), including one which simply involved pressing a button at paced intervals in the absence of any particular form of stimulation (Richer \& Beatty, 1985). The indication is that phasic LC activation is involved with motor preparation and execution, but the capacity in which it is involved and the exact function that it serves is not wholly clear.

In Experiments 4, 5 and 6, the pupil began to dilate up to $1000 \mathrm{~ms}$ before the committal of a button press. These findings corroborate similar observations from pupillometry studies in humans (e.g. Einhäuser et al., 2010; Richer \& Beatty, 1985) and map onto findings from neural recording studies in primates where phasic LC activation was observed prior to a motor response (e.g. Clayton et al., 2004; Rajkowski et al., 2004). In line with adaptive gain theory (Aston-Jones \& Cohen, 2005), this pupil-LC effect may reflect the consolidation of task-related decision processes, with NA release 
serving to enhance the responsivity of the cortical circuits involved in initiating the chosen cognitive or behavioural course of action. However, it seems that the largest contributing factor to the pupillometric effect of button pressing is not the decision process, but rather the motor act itself. When task-related decisions are made covertly and indicated with a button press at a later time, as in Einhäuser et al. (2010) and Experiment 2 of this thesis, the corresponding pupil dilation is much smaller than that which is seen at the actual time of button pressing. The neuroscience is not clear on why this might be the case, but Varazzani et al.'s (2015) observations in primates offer a potential explanation.

The primates in question were trained to perform a reward / effort task which required them to squeeze a bar with an amount of force indicated by a cue at the start of the trial. During this task, the firing rate of LC neurones around the time of action was highly correlated with pupil diameter following overt responses as well as the force with which those responses were made. This was the case even after removing the impact of task parameters, which implies that the motor component of the pupil-LC response exists over and above the requirements of the task. Varazzani et al. (2015) suggested that the phasic LC activation coextensive with pupil dilation and response force may be serving to mobilise both the physical (muscular) and physiological (autonomic) energy required to complete the action. Such an interpretation fits well with one previous pupillometry study in humans where pupil dilation scaled with the required actuation force of a button response (Richer \& Beatty, 1985), and it is generally consistent with research and theory implicating autonomic nervous activity in both physiological (Acevedo et al., 2007; Collet, Roure, Rada, Dittmar, \& Vernet-Maury, 1996) and cognitive effort (Howells, Stein, \& Russell, 2010; Kahneman \& Beatty, 1966; Webb et al., 2010).

\section{Visual search}

Each of the visual search experiments in this thesis demonstrated pupil dilation to taskrelated events such as stimulus appearance, target detection and response preparation and execution. In Experiment 1, the large dilations following stimulus exposure were explained best in terms of the combined effects of cognitive and stimulus related factors, but Experiments 2 and 4 demonstrated pupil responses to stimuli in the relative absence of methodological confounds. In particular, the small yet reliable dilations following stimulus presentation in Experiment 2, as well as those following fixations on targets in Experiment 4, seemed to reflect cognitive responses to the stimuli. The neuroscientific evidence set out in this thesis attributes these pupil dilations to underlying phasic activity 
in the LC-NA system, but the question remains as to the cognitive function this neural activity may be serving in these contexts.

Both visual search tasks were procedurally predictable and did not involve any sudden changes in stimulus parameters or contingencies, so it seems unfitting that the phasic LC-NA activity is serving as an unexpected uncertainty signal (Yu \& Dayan, 2005) or as an interrupt signal to facilitate the reconfiguration of cortical networks involved in the task (Bouret \& Sara, 2005). What is consistent between these two experiments is that pupil dilations were reliably observed in response to stimuli with high task-utility. In both experiments, participants were instructed to perform as accurately as possible. For Experiment 2, a two-alternative forced choice task with briefly presented stimuli, optimal performance depended on being prepared to make a correct judgement as to the presence or absence of a target on every trial, meaning each briefly appearing stimulus array had the same utility whether a target was present or not. In Experiment 4, optimal performance depended primarily on correctly identifying the number of targets that were present in an array, meaning task utility lay primarily with target stimuli. Adaptive gain theory (Aston-Jones \& Cohen, 2005) offers a fitting yet parsimonious interpretation for these overall patterns, namely that the phasic LC activity following the detection of behaviourally relevant stimuli is serving to energise the cortical circuits involved in task-specific decision processes (e.g. was a target present? am I looking at a target or a distractor?), thereby facilitating the correct behavioural or cognitive response (e.g. respond 'absent', add plus one to the running total of targets, terminate the search). Pursuing this line of thought, the phasic pupil responses observed during visual search do not reflect specific cognitive processes such as target detection or memory accumulation; rather they reflect transitions between cognitive states following the outcome of task-related decisions.

\section{Vigilance}

The two vigilance experiments in this thesis assessed behavioural and pupil measures during prolonged tasks requiring continuous successive visual target detection in the absence of any reinforcement. In both cases, a decrement in performance was observed across the whole task period, along with a corresponding decrease in the magnitude of task-related pupillary responses (i.e. all detection responses in Experiment 5 and hits, misses and false alarms in Experiment 6). This general pattern of results resonates strongly with previous findings in the pupillometry literature (e.g. Beatty, 1982b; Hopstaken, Van der Linden, Bakker, \& Kompier, 2015; Hopstaken, van der Linden, et al., 2015; Murphy et al., 2011; Unsworth \& Robison, 2016) and is what would be 
predicted by the adaptive gain theory of LC-NA function (Aston-Jones \& Cohen, 2005). Specifically, as task utility diminishes, as was inevitably the case in these monotonous and unrewarding tasks, the mode of LC output is driven from phasic to tonic, thereby promoting a transition from exploitative behaviour (e.g. task engagement, correct responses) to explorative behaviour (e.g. task unrelated thought, mind wandering). In line with Thomson et al.'s (2015) resource control theory of sustained attention, this shift in behavioural state may reflect a decline in executive control and the subsequent impaired ability to actively maintain task goals.

The reduction in phasic LC output in both vigilance experiments was marked by the declining task-evoked responses, but the corresponding rise in baseline pupil size that would also be predicted by adaptive gain theory-thought to reflect tonic discharge activity in the LC (e.g. see Rajkowski et al., 1993)—was not observed in the data. Baseline measures in Experiment 6 varied little throughout the task and those in Experiment 5 changed erratically within and between blocks without showing a reciprocal relationship with performance or task-evoked pupil measures. Further, in Experiment 5, there was no significant difference in baseline pupil size prior to trials with the best and worst performance. Though it is not what adaptive gain theory would predict, this lack of a consistent relationship between baseline pupil measures, task performance and task-evoked pupil responses is a common theme in the wider literature (e.g. Franklin et al., 2013; Grandchamp et al., 2014; Hopstaken, Van der Linden, Bakker, \& Kompier, 2015; Kristjansson et al., 2009; Mittner et al., 2014; Smallwood et al., 2012; Smallwood et al., 2011; Unsworth \& Robison, 2016; van den Brink et al., 2016; Van Orden et al., 2000, but see Gilzenrat et al., 2010). The inconsistency may in part be down to differences between studies with respect to the task requirements, but it could also be linked to the variable approaches used in obtaining and analysing baseline pupil measures in general. As a consequence, the measures of baseline pupil size in Experiments 5 and 6 do not fit neatly within existing theoretical frameworks, and may therefore not be a reliable indication of underlying cognitive processesing in these contexts.

\subsubsection{Theoretical conclusion}

Overall, the findings of this thesis support the notion that fluctuations in pupil size under conditions of constant luminance can serve as useful indices of cognitive processes involved in visual search and vigilance. Across all tasks, the most consistent finding was that of a relationship between task-evoked pupil responses and task utility, which is a central prediction of the adaptive gain theory of LC-NA function (Aston-Jones \& Cohen, 
2005). Within this framework, the task-evoked pupil dilations reflect coextensive LC activation which serves to facilitate behavioural or cognitive responses to the outcome of task-related decision processes by adjusting the gain of the cortical circuits involved. Under this conclusion, task-evoked pupil dilations are agnostic towards specific cognitive processes and simply reflect transitions between behavioural and cognitive states across the short and long terms. Inferences regarding specific cognitive functions such as attention and working memory and their associated cortical mechanisms must ultimately be made within the context of task demands, other dependant measures and established theory.

\subsection{Future research}

\subsubsection{Quantifying and correcting for the effects of motor-response force on pupil dilation}

One of the most consistent observations throughout this thesis and throughout the cognitive pupillometry literature in general is that motor acts, such as pressing a button to indicate some aspect of task fulfilment, contribute substantially to pupil dilation. This is problematic, as the robust motor component, which may exist over and above the demands of the task (Varazzani et al., 2015), can mask the diminutive cognitive effects which are of primary research interest. Although the pupillometric effects of motor acts are well-characterised, the extent to which the force with which they are committed affects the magnitude of the associated dilations is less clear. The studies by Richer and Beatty (1985) and Varazzani et al. (2015) mentioned above are the only two examples to the author's knowledge which speak to this issue, and on the basis of their results it seems reasonable to assume that there would be a continuous linear relationship between motor response force and pupil dilation. This hypothesis could be tested with the aid of a force-sensitive response box (e.g. Englund \& Patching, 2009) and a series of simple pupillometry experiments including various button-pressing and force-requiring conditions. If the effects of motor response force prove to be highly predictive of dilation magnitude, then force data could potentially serve as a control measure in the statistical analysis of pupil data, either as a covariate, or as a means of regressing the motor component from the observed pupil dilations. 


\subsubsection{Determining the precise neural mechanisms of the cognitive pupillary response}

This thesis has considered a broad range of evidence from behavioural, pharmacological, brain imaging, and single-unit recording studies which collectively points to a functional role for the LC in the cognitive modulation of pupil size. The most direct evidence for this proposition comes from the handful of single-unit recording studies in monkey which demonstrate a tight coupling between pupil size and spiking activity in LC neurones (Joshi et al., 2016; Rajkowski et al., 1993; Varazzani et al., 2015). These findings are widely cited in the cognitive pupillometry literature as evidence that instantaneous, nonluminance-mediated fluctuations in pupil size reflect the current activity of noradrenergic neurones in the LC (e.g. Laeng et al., 2012). However, as noted by Costa and Rudebeck (2016), the studies in question were correlational in nature and therefore do not show that activity in the LC is causally related to pupil size. The authors further pointed out that there is generally much uncertainty surrounding the precise neural mechanisms of the pupil-LC relationship and highlighted the possibility that the tight temporal coupling could be mediated by indirect mechanisms, such as input from a common source into both the LC and pupillomotor nuclei (e.g. the nucleus paragigantocellularis of the ventral medulla), or interactions between the LC and cortical areas involved in autonomic arousal (e.g. the anterior cingulate cortex). Further research to explore these possibilities would clearly be beneficial to cognitive pupillometry in general.

\subsection{Final remarks}

This thesis has examined methodological and theoretical issues in cognitive pupillometry and demonstrated the effective application of this technique in laboratory studies of visual search and vigilance. The findings from six original experiments have contributed to the wider psychological research literature and affirmed the utility of pupillometry, both as a tool for gaining insight into cognitive function and as a means of achieving practical aims in applied settings. However, both the literature and the original experiments detailed herein emphasise the constraints on scope arising from a range of methodological and theoretical limitations. Further research, together with technological, methodological and theoretical advances will be necessary before real-time pupillometry either alone or in conjunction with other physiological measures, can be used to improve the outcomes of socially important tasks which involve elements of visual search, vigilance, or both. 



\section{Appendix A}

\section{Experiment 0: Measuring the PLR}

\section{Introduction}

The purpose of the current experiment was to examine the PLR, an autonomous biological function that serves to protect photoreceptors and optimize visual acuity by mediating the amount and trajectory of light that reaches the retina (Campbell \& Gregory, 1960; Laughlin, 1992; Woodhouse, 1975). It is a well-known fact that the magnitude of pupil constriction in response to a light stimulus is proportional to the strength of the stimulus (Ellis, 1981; Loewenfeld, 1993; Reeves, 1920). The present experiment sought to replicate this finding by subjecting participants to three levels of luminance on a computer display in a darkened room. It was predicted that the magnitude of pupil constriction would be proportional to the strength of the luminance stimulus. The data from this experiment were used to develop and test methods for processing and analysing pupillometry data in the early stages of the research program.

\section{Method}

\section{Participants}

Thirteen participants ( 9 females; age range 20-39 years, $M=28.38, S D=5.55$ ) completed the experiment voluntarily. All participants were students at Swansea University reporting normal or corrected-to-normal acuity and colour vision. The experimental protocol was approved by the Ministry of Defence Research Ethics Committee and the Department of Psychology Ethics Committee at Swansea University. Written informed consent was obtained from each participant. 


\section{Design}

Participants were exposed to brief luminance increments in a repeated-measures design, with Luminance Level $(1,2,3)$ being the only experimental manipulation of interest. Eighty-one trials were completed ( 9 blocks of 9 trials) in a single session that lasted approximately thirty-five minutes. Participants fixated a target stimulus as it appeared at various locations on the screen and maintained fixation as the luminance stimulus was presented. There were 9 locations in total (equidistant on a virtual grid spanning the whole screen), and the target appeared at each location once in every block.

\section{Stimuli and apparatus}

The stimuli used to evoke the PLR were white circles surrounding a white fixation cross on a black background (Figure A.1). Three linearly increasing levels of luminance were implemented by changing the number of white-filled circles: 2 circles for Luminance Level $1\left(8.7 \mathrm{~cd} / \mathrm{m}^{2}\right), 4$ circles for Level $2\left(13.6 \mathrm{~cd} / \mathrm{m}^{2}\right)$ and 6 circles for Level $3(19.6$ $\mathrm{cd} / \mathrm{m}^{2}$ ). Luminance was measured using a colorimeter (ColorCAL MKII, Cambridge Research Systems). Three equally prevalent rotational variants (not shown) were used for Levels 1 and 2 in order to counter any effects caused by the uneven distribution of light on the retina.

Stimuli were presented on an LG Flatron F900p monitor with a pixel resolution of $1024 \times 768$ and a vertical refresh rate of $60 \mathrm{~Hz}$. At a distance of $60 \mathrm{~cm}$ from the screen, each individual circle subtended approximately $0.8^{\circ}$ of visual angle, and the overall stimulus spanned $2.5 \times 2.5$ degrees. Pupil size of the right eye was recorded on an EyeLink 1000 system (SR Research Ltd., Ontario, CA) sampling at $1000 \mathrm{~Hz}$. The experiment was programmed in Experiment Builder (SR Research Ltd., Ontario, CA).

\section{Procedure}

Participants were instructed always to fixate the cross at the centre of the target stimulus and to avoid moving their eyes during experimental trials. A nine-point calibration was performed at the beginning of the experiment and further calibrations were performed as required throughout. Each trial began with a drift check at the centre of the screen where participants fixated only the white cross from the centre of the target. After this, the target stimulus appeared at one of nine locations on the screen, prompting the participant to fixate it. The luminance increment was displayed for $500 \mathrm{~ms}$ once the participant's gaze was detected for $2750 \mathrm{~ms}$ within a rectangular interest area surrounding the target $\left(4^{\circ} \times 4^{\circ}\right.$ visual angle, centred on the fixation cross). Following the presentation of the 


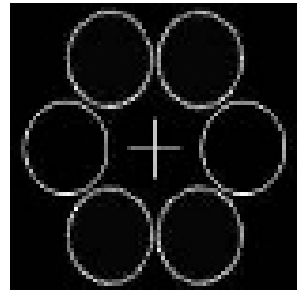

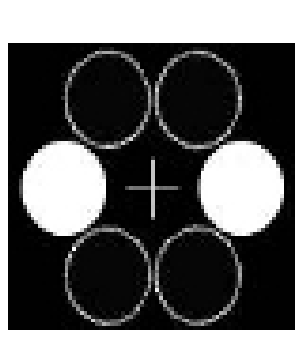

Level 1

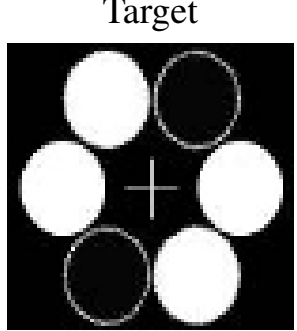

Level 2

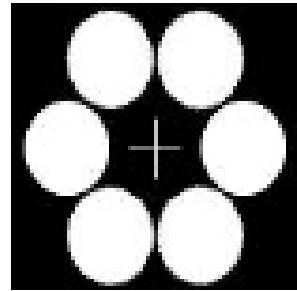

Level 3

Figure A.1. The target and luminance stimuli used to evoke the pupillary light reflex (rotational variants not shown).

luminance increment, the target remained in the same location for a further $2750 \mathrm{~ms}$ while the pupil response was measured. The trial cycle is displayed in Figure A.2.

\section{Data processing and analysis}

Pupil data were preprocessed using the methods described in Chapter 3. Eye blinks were reconstructed using linear interpolation, and the data was smoothed using a third-order Butterworth filter (4 Hz cut-off). Epochs of $3500 \mathrm{~ms}$ were extracted for each trial, from $500 \mathrm{~ms}$ before the onset of the luminance increment to $3000 \mathrm{~ms}$ afterwards. Individual trials were rejected if a blink occurred during the luminance increment, or if more than $40 \%$ of the data had to be interpolated. Nonparametric permutation tests were used to assess the significance of modulations from the baseline period (one-sample $t$-tests), and the effect of Luminance Level ( $F$-tests). Significance threshold were determined theoretical from the respective distributions under the appropriate degrees of freedom.

\section{Results}

As predicted, pupils constricted following exposure to the luminance stimuli and the magnitude of constriction increased as a function of Luminance Level. The grandaverage traces for each Luminance Level are shown in Figure A.3, together with the results of the nonparametric permutation tests showing significant modulation from 


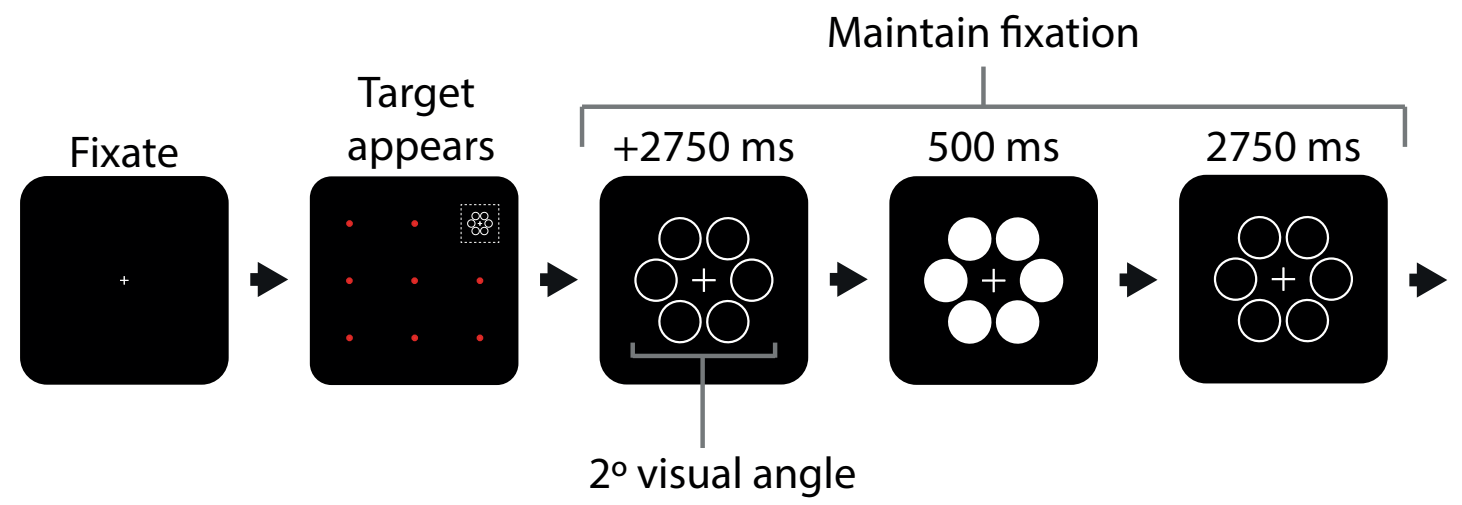

Figure A.2. The trial sequence of Experiment 0. Participants maintained central fixation until the target stimulus appeared at one of nine locations on the screen. After fixating the target for $2750 \mathrm{~ms}$, the luminance increment was presented for $500 \mathrm{~ms}$, and then fixation was maintained for a further $2750 \mathrm{~ms}$.

baseline and the significant main effect of Luminance Level. Follow-up $t$-tests conducted on the average pupil modulation within the window revealed significant differences between all level of luminance (all $p$ s <.05).

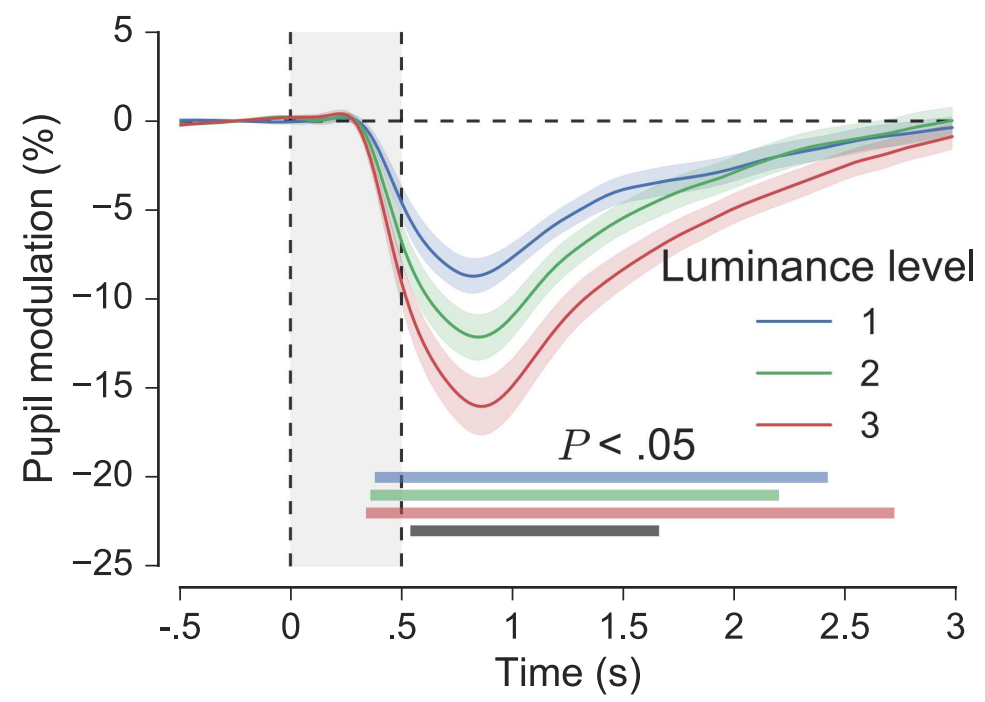

Figure A.3. Grand average pupil responses to stimuli with three incrementing levels of luminance. The light-grey shaded area spanning the $y$-axis denotes the onset and duration of the luminance increment, and the shaded areas surrounding the pupil traces show the SEM. The horizontal coloured bars show, for each luminance level, significant change from baseline as identified by non-parametric permutation tests (1024 permutations, $p<.05$, cluster-corrected). The dark horizontal line denotes the period at a significant main effect was present $(F$-test, 1024 permutations, $p<.05)$. 


\section{Discussion}

Consistent with previous findings (e.g. Ellis, 1981; Loewenfeld, 1993; Reeves, 1920) and widely-held knowledge about the general functionality of the human eye, pupillary constriction in the current experiment scaled with the strength of the luminance stimuli. It is important to note that the observed effects of pupillary constriction, which ranged from between $9 \%$ to $17 \%$ peak modulation, are much larger in magnitude than the dilatory effects associated with cognition, which were the focus of this thesis. However, the data from the current experiment were useful in developing the methods that were used to process and analyse pupillometry data in Chapters 4-6. 

Appendix B

Departmental ethics applications 


\section{Department of}

Psychology

ETHICS

COMIMITTEE

\section{Memo}

\begin{tabular}{|c|c|}
\hline To: & Joel Martin \\
\hline \multirow[t]{2}{*}{ From: } & Professor Andy Parrott \\
\hline & for Departmental Ethics Committee \\
\hline Copy: & Dr. Simon Dymond \\
\hline Date: & $22^{\text {nd }}$ September, 2014 \\
\hline Re: & $\begin{array}{l}\text { Exploring Target and Threat Detection } \\
\text { with Eye Tracking and Pupillometry }\end{array}$ \\
\hline
\end{tabular}

Your proposed study "Exploring Target and Threat Detection with Eye Tracking and Pupillometry", has been reviewed and is approved. Provided that the information obtained is kept absolutely confidential and that no personally identifiable information is entered on computer, you may proceed with your studies.

Please ensure that the signed copy of this Ethical Approval, together with any other paperwork associated with your research, is included in your final write up.

In order for your study to be displayed on the Experiment Management System (Participant Pool):

Forward this approval via email to Dr. Irene Reppa

$\underline{\text { AND }}$

Send a request for your study to be made visible, via the link on the EMS website (see Researcher Documentation for details). 
Application for Risk Assessment and Ethical Approval PLEASE COMPLETE THE FORM USING TYPESCRIPT (hand-written applications will not be considered)

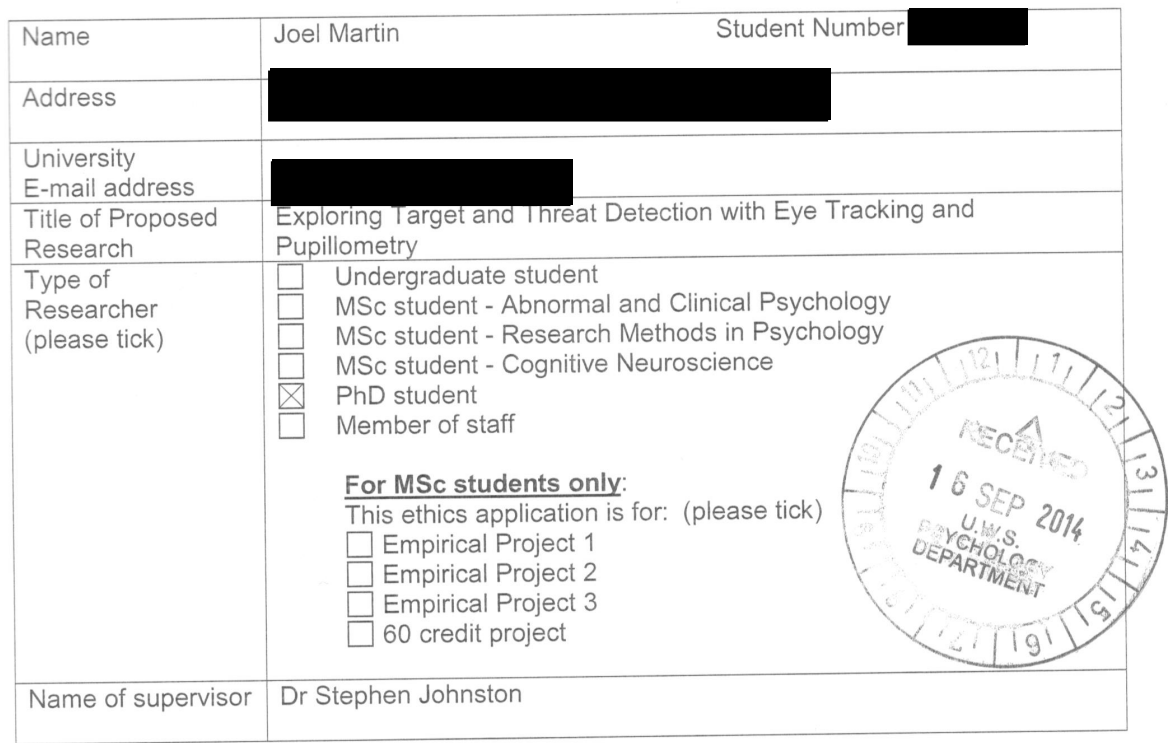

1. Briefly describe the main aims of the research you wish to undertake. Please use nontechnical language wherever possible.

1) To Explore behavioral and ocular responses to potentially threatening target stimuli in various viewing conditions with a view to devising performance improvement methods that can be utilized in everyday action

2) To assess the practicability of using pupillometry as an index for target detection, even when perception of the stimuli is not acknowledged with a behavioral response

3) To explore the effect of participant fatigue induced by sleep deprivation on the integrity of the behavioral and ocular response characteristics identified.

Further details in Section 8 of the attached MoDREC proposal.

2. Briefly describe the overall design of the project

This project involves a series of 8 Experiments:

Experiments 1-2: X-ray Baggage Screening

Experiments 3-5: Visual Search / Target Detection Tasks

Experiments 6-7: Constant Vigilance Task

Experiment 8: Sleep Deprivation Study

Comprehensive details on the overall design of this project can be found in Section 9 of the attached MoDREC proposal.

3. Briefly describe the methods of data collection and analysis. Please describe all measures to be employed (e.g., questionnaire responses, reaction times, accuracy, skin conductance responses, etc.). If questionnaire or interviews are to be used, please provide the questionnaire / interview questions and schedule.

Pupil diameter and eye-tracking data will be obtained with an SR Research EyeLink-1000 eye- 
tracking system. Sleep questionnaires will be used in Experiment 8. Behavioural responses (e.g. button presses) are to be collected on computer keyboards. Data will be analysed using

parametric statistical techniques, such as analysis of variance (ANOVA) and the student's t-test. (See Section 8 of attached proposal.)

4. Location of the proposed research (i.e., Departmental labs, schools, etc)

The eye-tracking lab and sleep lab in The Department of Psychology, Swansea University Campus.

5. Describe the participants: give the age range, gender, inclusion and exclusion criteria, and any particular characteristics pertinent to the research project.

Participants will be students at Swansea University and members of the general public, between the ages of $18-65$ years. For all experiments will only recruit participants who report that they:

- Have normal or corrected-to-normal vision

- Have not been diagnosed as having a psychological or neurological disorder

Additionally for the sleep deprivation study:

- Do not take recreational drugs or excessive alcohol (defined as intake greater than 6 units on a night or greater than 21 units per week)

- Are prepared to restrict daily alcohol and caffeine intake to within government recommended guidelines throughout their participation (alcohol: 2-3 units for woman, 3-4 units for men; caffeine: $200 \mathrm{mg}$ - roughly equivalent to 3 cups of tea or 2 cups of instant coffee)

- Do not have any University, workplace or pastoral commitments which they think could be negatively affected by taking part in the study

- Will not be training for any major sporting or fitness events, such as a half marathon, a long distance bike ride or a competitive sports tournament

- Do not report having been diagnosed with a psychiatric, neurological or sleep-related disorder

- Have not worked night-shifts in the previous year

- Have not travelled through more than one time-zone in the two month period before the proposed participation date

- Are not an extreme morning or evening person, as assessed by the MorningnessEveningness questionnaire (Horne \& Ostberg, 1975)

Further details in Section 9 of attached form. Also, see Appendix 2.a and 2.b for screening questionnaires.

6. How will the participants be selected and recruited?

Subject pool.

General public

Other. Please give details:

\begin{tabular}{|c|c|c|c|}
\hline \multirow[t]{2}{*}{7.} & \multirow{2}{*}{$\begin{array}{l}\text { Will the study be advertised outside the Department's Electronic } \\
\text { Subject Pool system (e.g. via a poster or email notice)? }\end{array}$} & Yes & $\triangle$ \\
\hline & & No & $\square$ \\
\hline
\end{tabular}

advertisement to this Ethics Form:

Wording for poster advertising Experiments 1-7 
"We are seeking healthy participants aged 18-65 years to participate in a research study that is being conducted in The Department of Psychology at Swansea University. You will be required to complete a series of computerized cognitive tasks as eye-tracking and pupil-size measures are acquired. Participation should take no more than 1 hour and you will receive $£ 7$ compensation for your time. Please send an email to Joel Martin for further information. You can scan the QR code to do this directly, or take a contact slip and do it later."

Wording for poster advertising Experiment 8

"Would you be willing to participate in a sleep deprivation study? A team of Researchers at Swansea University are conducting an investigation into the effects of sleep loss on cognitive task performance. We seek interested volunteers aged 18-65 who would be prepared to spend two nights (a week apart) in the sleep laboratory at Swansea University. In one session you will be permitted to sleep, whereas in the other you will be kept awake. Before and after each session you will be required to engage with two computerized tasks as eye-tracking measures are obtained. You will also be asked to complete a number of questionnaires as part of the research. Food will be supplied during each stay and you will be paid $£ 100$ for your participation. Please send an email to Joel Martin for further information. You can scan the QR code to do this directly, or take a contact slip and do it later."

See posters in the appendices of attached MoDREC application form (Appendix 4).

8. What procedures (e.g., interviews, computer-based learning tasks, etc.) will be carried out on the participants?

Participants will engage with simple computer-based tasks as eye-tracking measurements are simultaneously acquired. In Experiment 8, participants will undergo two sessions in the sleep laboratory (a sleep deprivation session and a normal sleep session) and two weeks of wrist actigraphy and sleep-diary keeping. Comprehensive details in MoDREC application form.

9. What potential risks to the participants do you foresee and how do you propose to ameliorate/deal with potential risks? For instance, provide contact details of Student Counseling services and relevant community support organizations, etc.

There are risks associated with extreme tiredness which may affect participants who are subject to the sleep deprivation protocol in Experiment 8. A taxi home will be provided after the intervention and a follow-up phone call will be made the following day to make sure participants are not suffering any serious side-effects. More details can be found in the MoDREC application form. A safety committee has been appointed to oversee safety issues relating to the sleep deprivation study.

10. What potential risks to the interests of the researchers do you foresee and how will you ameliorate/deal with potential risks?

There are no anticipated risks to the interests of the researchers in this experiment.

11. How will you brief and debrief participants? (Please attach copy of debrief information to be given to participants)

Participants will be briefed and debriefed, verbally, by the experimenter. See also participant information sheets and debrief forms in the Appendices of the MoDREC application form.

\begin{tabular}{|l|l|l|}
\hline $\begin{array}{l}\text { 12. } \begin{array}{l}\text { Will informed consent be sought from } \\
\text { participants? }\end{array} \\
\text { If no, please explain below: }\end{array}$ & $\begin{array}{l}\text { Yes (Please attach a copy of } \\
\text { the consent form) }\end{array}$ & $\square$ \\
& No & $\square$ \\
\hline
\end{tabular}




\begin{tabular}{l}
$\begin{array}{l}\text { 13. If there are doubts about participants' abilities to give informed consent, what steps have } \\
\text { you taken to ensure that they are willing to participate? }\end{array}$ \\
\hline Participants will be aged $18+$ years and therefore able to provide written informed consent . \\
\end{tabular}

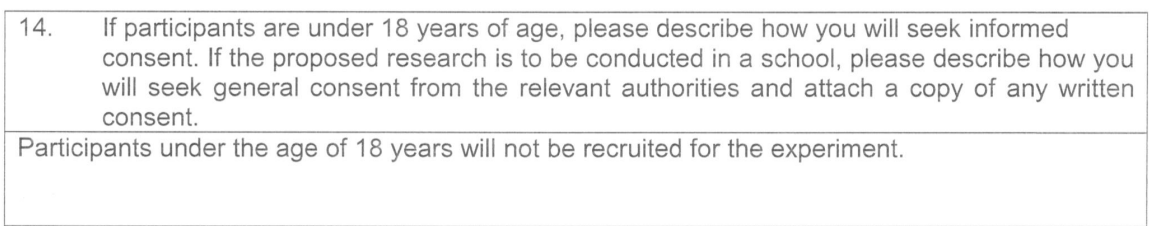

\begin{tabular}{|l|}
\hline 15. How will consent be recorded? \\
\hline On a written consent form. \\
\hline
\end{tabular}

\begin{tabular}{|l|l|}
\hline $\begin{array}{l}\text { 16. Will participants be informed of the right to withdraw without } \\
\text { penalty? }\end{array}$ & Yes \\
\cline { 2 - 2 } If no, please detail the reasons for this: & \\
\hline
\end{tabular}

17. How do you propose to ensure participants' confidentiality and anonymity?

Password protected computers will be used to store data files and participants will be assigned a unique subject ID (e.g. "subject_1") from which they will not be identifiable to anyone but the researchers involved with data collection.

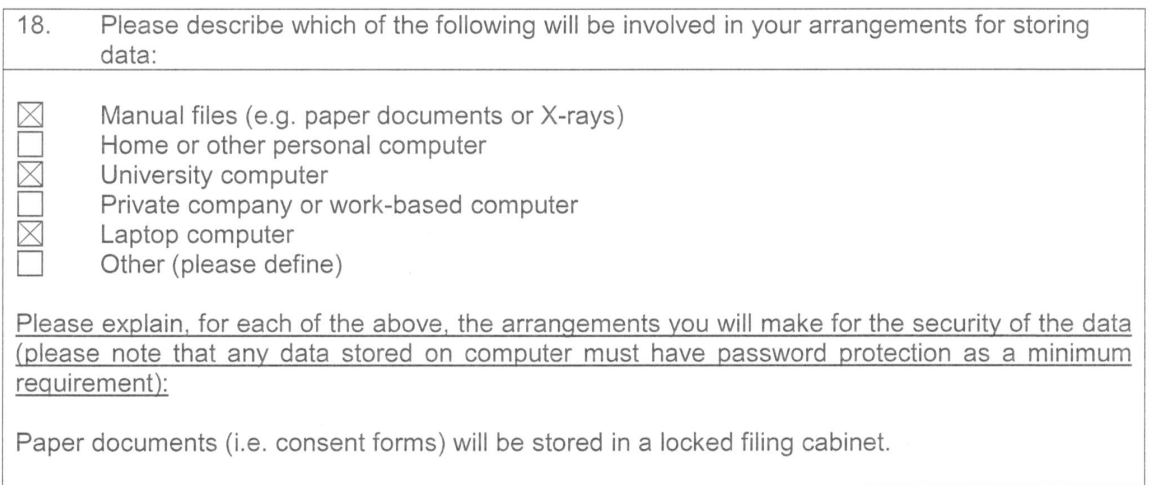

\begin{tabular}{|l|l|}
\hline 19. Will payments or subject pool credits be made to participants? & Yes \\
\cline { 2 - 2 } & No \\
\hline If yes, please specify quantities involved (e.g., £5 or 1 hour credits): \\
£7 or 1 hour credits will be awarded to participants in Experiments 1-7. \\
Participants in Experiment 8 will be awarded $£ 100$ upon completion of the protocol. \\
\hline
\end{tabular}




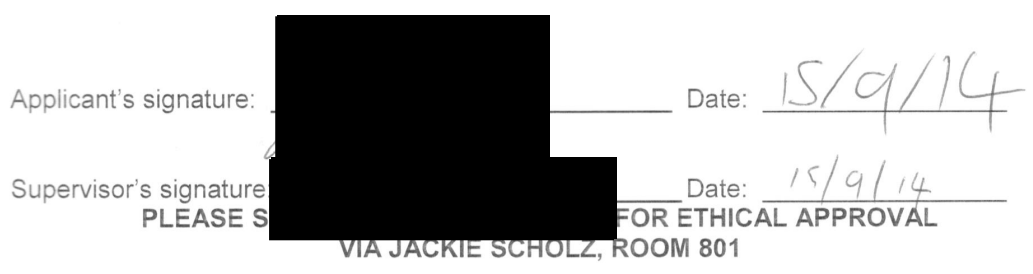

**RESEARCH MAY ONLY COMMENCE ONCE ETHICAL APPROVAL HAS BEEN OBTAINED ** 
CHECKLIST OF ATTACHMENTS:

PLEASE REMEMBER TO ATTACH COPIES OF EACH OF THE FOLLOWING

(WHERE RELEVANT)

*INCOMPLETE APPLICATIONS WILL NOT BE CONSIDERED*

$\triangle$ Copy of Participant Information Sheet

Copy of Consent Form

$\triangle$ Copy of Participant debrief

$\square$ Copy of any questionnaires and/or interview schedules to be employed

$\square$ Copy of written consent from local authorities (e.g., schools)

If your proposed research is with 'vuinerable' groups (e.g., children, people with developmental disorder), please attach a copy of your clearance letter from the Criminal Records Bureau (if UK) or equivalent non-UK clearance.

As a member of the Departmental Ethics Committee, I have scrutinized this application. In my opinion some elements are under-developed and require attention. The application is therefore returned to you as the student's supervisor.

Departmental Ethics Committee Use Only

Members of the Departmental Ethics Committee have considered the ethical issues raised by this project, and have the following comments:

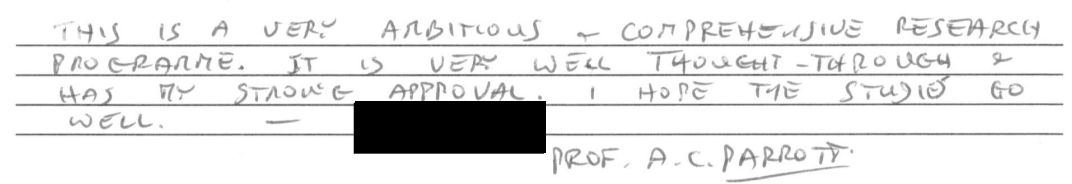

Please ensure that you take account of these comments and prepare a revised submission that should be either shown to your supervisor (if you are an undergraduate or postgraduate student) or resubmitted (if you are a member of staff) to the Departmental Ethics Committee.

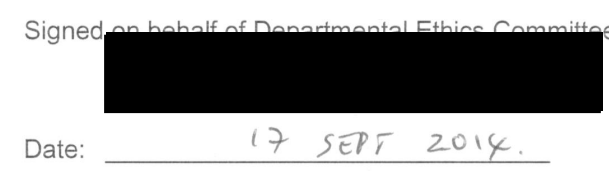

Were there any significant safety issues that needed resolving?

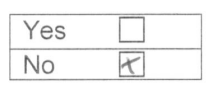

If YES:

College Safety Officer Approval (Mark Hughes): 
Date:

NOTE TO LEVEL 3 UNDERGRADUATE PROJECT STUDENTS, MSC and RESEARCH POSTGRADUATE STUDENTS:

PLEASE INCLUDE A SIGNED COPY OF YOUR ETHICS FORM \& CONFIRMATION LETTER OF APPROVAL AS AN APPENDIX IN YOUR FINAL YEAR PROJECT OR THESIS. 
Department of

Psychology

ETHICS

COMMITTEE

\section{Memo}

$\begin{array}{ll}\text { To: } & \begin{array}{l}\text { Joel Martin } \\ \text { Student Number(s): }\end{array} \\ \text { From: } & \begin{array}{l}\text { Dr. Jeremy Tree } \\ \text { for Departmental Ethics Committee }\end{array} \\ \text { Copy: } & \text { Dr. Stephen Johnston } \\ \text { Date: } & 4^{\text {th }} \text { November, } 2013 \\ \text { Re: } & \begin{array}{l}\text { Characterizing the effects of gaze direction on } \\ \text { the luminance elicited pupillometric response }\end{array}\end{array}$

Your proposed study, "Characterizing the effects of gaze direction on the luminance elicited pupillometric response", has been reviewed and is approved. Provided that the information obtained is kept absolutely confidential and that no personally identifiable information is entered on computer, you may proceed with your studies.

Please ensure that the signed copy of this Ethical Approval, together with any other paperwork associated with your research, is included in your final write up.

In order for your study to be displayed on the Experiment Management System (Participant Pool):

Forward this approval via email to Dr. Irene Repp

$\underline{\text { AND }}$

Send a request for your study to be made visible, via the link on the EMS website (see Researcher Documentation for details). 
Application for Risk Assessment and Ethical Approval PLEASE COMPLETE THE FORM USING TYPESCRIPT

(hand-written applications will not be considered)

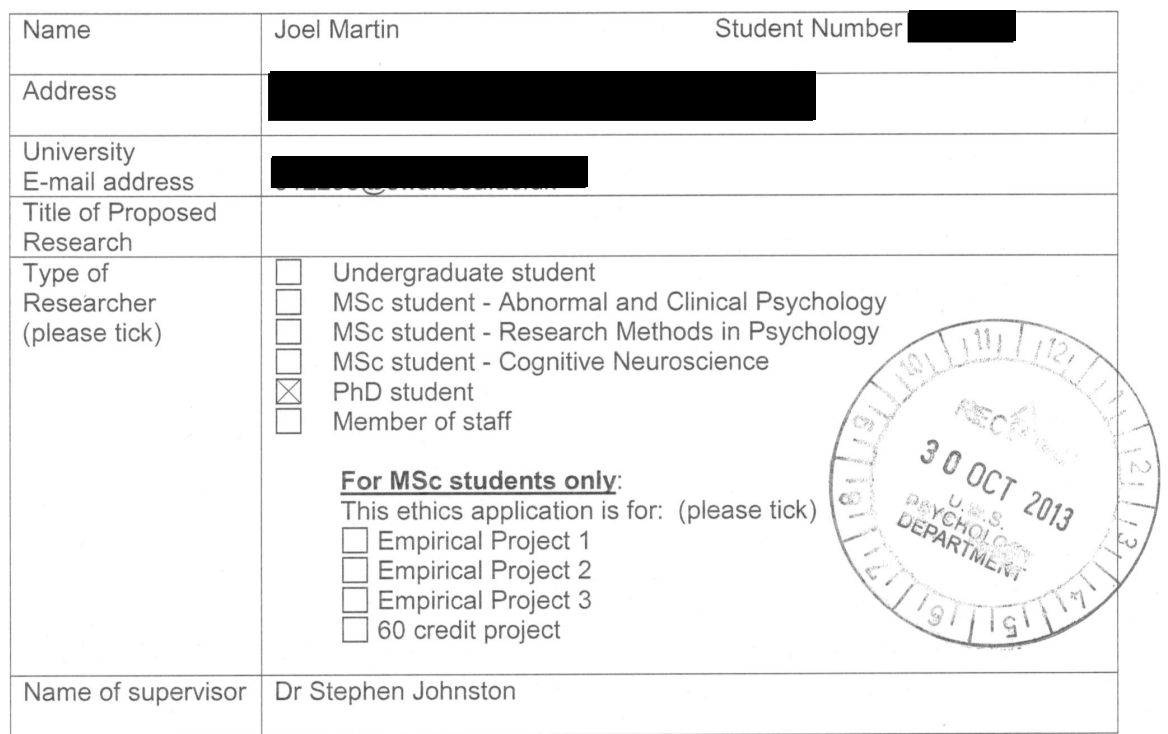

1. Briefly describe the main aims of the research you wish to undertake. Please use nontechnical language wherever possible.

To characterize the effects of gaze direction on the luminance elicited pupillometric response

2. Briefly describe the overall design of the project

This single session eye tracking experiment will employ a between subjects design. The data collection will take place in the eye-tracking laboratory at Swansea University $\left(8^{\text {th }}\right.$ floor of Vivian Tower). The duration of the experiment is expected to be no more than 1 hour.

In the experiment, participants will be asked to fixate upon a stimulus - a white cross surrounded by six circles. The stimulus will appear intermittently at various positions on the screen and at varying degrees of luminosity. The participant's task will be to track the stimulus and maintain fixation upon the central white cross.

This is purely a gaze contingent experiment. Other than fixating at the appropriate area of the screen, there will be no other behavioral demands upon the participant.

3. Briefly describe the methods of data collection and analysis. Please describe all measures to be employed (e.g., questionnaire responses, reaction times, accuracy, skin conductance responses, etc.). If questionnaire or interviews are to be used, please provide the questionnaire / interview questions and schedule.

Pupil diameter and gaze direction will be measured with an eye-tracking system (SR Research:

EyeLink-1000) 
4. Location of the proposed research (i.e., Departmental labs, schools, etc)

Within the eye-tracking laboratory on the $8^{\text {th }}$ floor of Vivian Tower

5. Describe the participants: give the age range, gender, inclusion and exclusion criteria, and any particular characteristics pertinent to the research project.

Participants will be students at Swansea University (aged 18+ years)

6. How will the participants be selected and recruited?

$\bigotimes$ Subject pool

General public

Other. Please give details:

7. Will the study be advertised outside the Department's Electronic

Subject Pool system (e.g. via a poster or email notice)?

Yes

No

If yes, please provide the wording of the advertisement here (or attach a copy of the intended advertisement to this Ethics Form):

8. What procedures (e.g., interviews, computer-based learning tasks, etc.) will be carried out on the participants?

Participants will engage with a simple gaze-contingent computer based task as eye-tracking measurements are simultaneously acquired

9. What potential risks to the participants do you foresee and how do you propose to ameliorate/deal with potential risks? For instance, provide contact details of Student Counseling services and relevant community support organizations, etc.

There are no anticipated risks to participants of this experiment

10. What potential risks to the interests of the researchers do you foresee and how will you ameliorate/deal with potential risks?

There are no anticipated risks to the researchers involved with this experiment

11. How will you brief and debrief participants? (Please attach copy of debrief information to

given to participants

Participants will be issued with a written brief and debrief of the experiment (example copies attached)

12. Will informed consent be sought from participants?

Yes (Please attach a copy of the consent form)

If no, please explain below:

No 


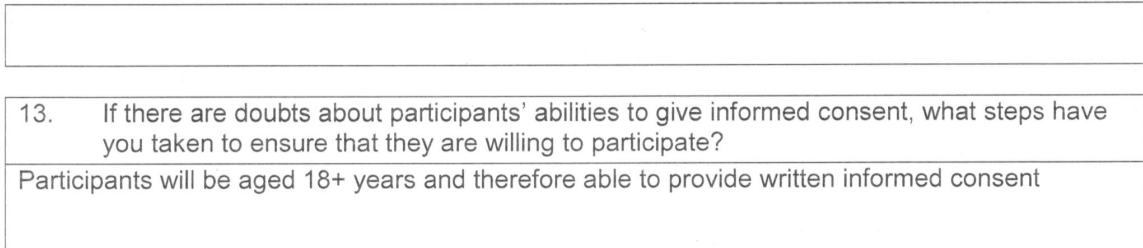

14. If participants are under 18 years of age, please describe how you will seek informed
consent. If the proposed research is to be conducted in a school, please describe how you
will seek general consent from the relevant authorities and attach a copy of any written
consent.

15. How will consent be recorded?

On a written consent form

\begin{tabular}{|l|l|}
\hline $\begin{array}{l}\text { 16. Will participants be informed of the right to withdraw without } \\
\text { penalty? }\end{array}$ & Yes \\
\cline { 2 - 2 } & No \\
\hline If no, please detail the reasons for this: & \\
\hline
\end{tabular}

17. How do you propose to ensure participants' confidentiality and anonymity?

Password protected computers will be used to store data files and participants will be assigned a unique subject ID (e.g. "subject_1") from which they will not be identifiable to anyone but the researchers involved with data collection

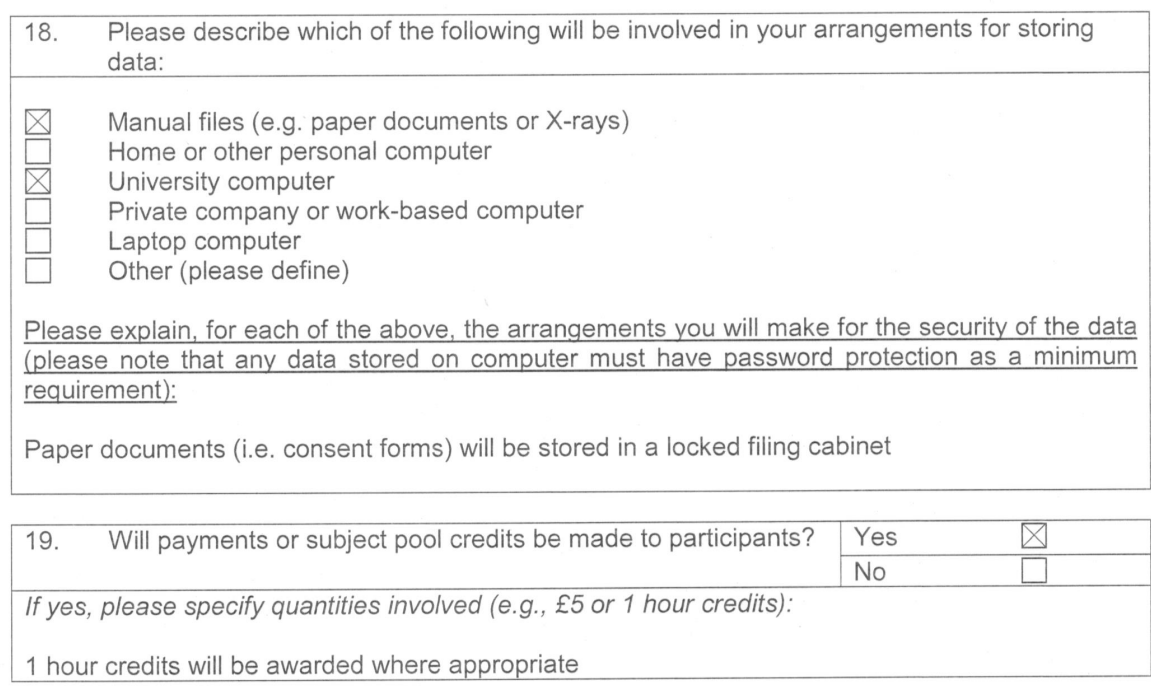




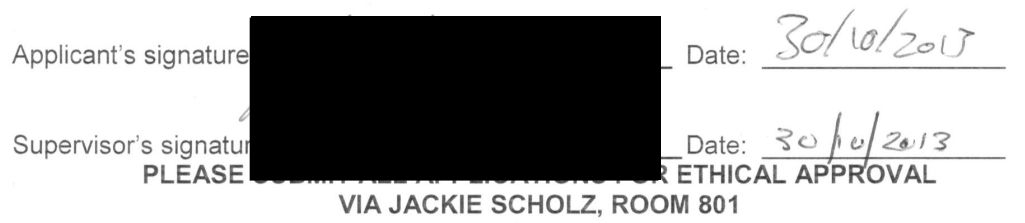

**RESEARCH MAY ONLY COMMENCE ONCE ETHICAL APPROVAL HAS BEEN OBTAINED** 
Date:

NOTE TO LEVEL 3 UNDERGRADUATE PROJECT STUDENTS, MSC and RESEARCH PLEASE INCLUDE A SIGNED COPY OF YOUR ETHICS

IONFIRMATION LETTER OF APPROVAL AS AN APPENDIX IN YOUR FINAL YEAR PROJECT OR THESIS. 
CHECKLIST OF ATTACHMENTS:

PLEASE REMEMBER TO ATTACH COPIES OF EACH OF THE FOLLOWING (WHERE RELEVANT)

*INCOMPLETE APPLICATIONS WILL NOT BE CONSIDERED*

\ Copy of Participant Information Sheet

Copy of Consent Form

$\triangle$ Copy of Participant debrief

Copy of any questionnaires and/or interview schedules to be employed

Copy of written consent from local authorities (e.g., schools)

If your proposed research is with 'vulnerable' groups (e.g., children, people with developmental disorder), please attach a copy of your clearance letter from the Criminal Records Bureau (if UK) or equivalent non-UK clearance.

As a member of the Departmental Ethics Committee, I have scrutinized this application. In my opinion some elements are under-developed and require attention. The application is therefore returned to you as the student's supervisor.

Departmental Ethics Committee Use Only

Members of the Departmental Ethics Committee have considered the ethical issues raised by this project, and have the following comments:

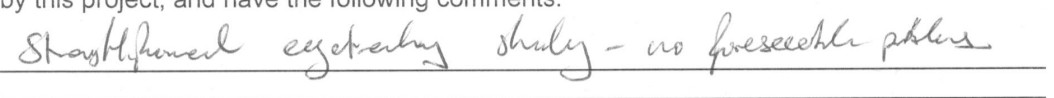

Please tick if

appropriate

Please ensure that you take account of these comments and prepare a revised submission that should be either shown to your supervisor (if you are an undergraduate or postgraduate student) or resubmitted (if you are a member of staff) to the Departmental Ethics Committee.

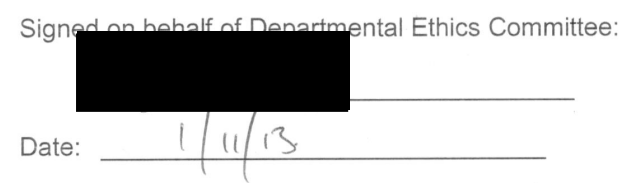

Were there any significant safety issues that needed resolving?

\begin{tabular}{|l|}
\hline Yes $\square$ \\
\hline No $\square$
\end{tabular}

If YES:

College Safety Officer Approval (Mark Hughes) 


\section{References}

Aboyoun, D. C. \& Dabbs, J. M. (1998). The Hess pupil findings: Sex or novelty? Social Behavior and Personality: an International Journal, 26(4), 415-419. doi:10.2224/ sbp.1998.26.4.415

Acevedo, E. O., Kraemer, R. R., Kamimori, G. H., Durand, R. J., Johnson, L, G., \& Castracane, V. D. (2007). Stress hormones, effort sense, and perceptions of stress during incremental exercise: An exploratory investigation. Journal of Strength and Conditioning Research, 21(1), 283-288. doi:10.1016/j.apergo.2009.08.003

Acland, B. T. \& Braver, T. S. (2014). Cili (v0.5.3) [computer software]. Retrieved from https://githib.com/beOn/cili

Adamo, S. H., Cain, M. S., \& Mitroff, S. R. (2013). Self-induced attentional blink. Psychological Science, 24(12), 2569-2574. doi:10.1177/0956797613497970

Adams, J. A. (1956). Vigilance in the detection of low-intensity visual stimuli. Journal of Experimental Psychology, 52(3), 204. doi:10.1037/h0048556

Adams, J. A. (1987). Criticisms of vigilance research: A discussion. Human Factors, 29(6), 737-740. doi:10.1177/001872088702900612

Ahern, S. \& Beatty, J. (1979). Pupillary responses during information processing vary with Scholastic Aptitude Test scores. Science, 205(4412), 1289-1292. doi:10. 1126/science. 472746

Ahern, S. \& Beatty, J. (1981). Physiological evidence that demand for processing capacity varies with intelligence. In Intelligence and learning (pp. 121-128). Springer US.

Ahlstrom, U. \& Friedman-Berg, F. J. (2006). Using eye movement activity as a correlate of cognitive workload. International Journal of Industrial Ergonomics, 36(7), 623-636. doi:10.1016/j.ergon.2006.04.002

Alexandridis, E., Leendertz, J. A., \& Barbur, J. L. (1991). Methods for studying the behaviour of the pupil. Journal of Psychophysiology, 5(3), 223-239. Retrieved from http://psycnet.apa.org/record/1992-11914-001 
Alnaes, D., Sneve, M. H., Espeseth, T., Endestad, T., van de Pavert, S. H. P., \& Laeng, B. (2014). Pupil size signals mental effort deployed during multiple object tracking and predicts brain activity in the dorsal attention network and the locus coeruleus. Journal of Vision, 14(2014), 1-20. doi:10.1167/14.4.1

Alvarez, G. A. \& Cavanagh, P. (2004). The capacity of visual short-term memory is set both by visual information load and by number of objects. Psychological Science, 15(2), 106-111. doi:10.1167/2.7.273

Anderson, B. (2011). There is no such thing as attention. Frontiers in Psychology, 2(SEP), 1-8. doi:10.3389/fpsyg.2011.00246

Andrea, K., Furdea, A., Halder, S., Hammer, E. M., Nijboer, F., \& Kotchoubey, B. (2009). A brain-computer interface controlled auditory event-related potential (P300) spelling system for locked-in patients. Disorders of Consciousness, 100(1157), 90-100. doi:10.1111/j.1749-6632.2008.04122.x

Andreassi, J. (2000). Pupillary response and behavior. In Human behavior and physiological response (pp. 289-307). Psychology Press. Retrieved from http://scholar. google.com/scholar?hl=en\%7B\%5C\&\%7DbtnG=Search\%7B\%5C\&\%7Dq= intitle:Pupillary+Response+and+Behavior\%7B\%5C\#\%7D0

Ariga, A. \& Lleras, A. (2011). Brief and rare mental "breaks" keep you focused: Deactivation and reactivation of task goals preempt vigilance decrements. Cognition, 118(3), 439-443. doi:10.1016/j.cognition.2010.12.007

Arrabito, G., Abel, S., \& Lam, K. (2007). Methods for mitigating the vigilance decrement in an auditory sonar monitoring task: a research synthesis. Canadian Acoustics, 35(1950), 15-23. Retrieved from http://jcaa.caa-aca.ca/index.php/jcaa/ article/view/1976

Astafiev, S. V., Snyder, A. Z., Shulman, G. L., \& Corbetta, M. (2010). Comment on "Modafinil shifts human locus coeruleus to low-tonic, high-phasic activity during functional MRI" and "Homeostatic sleep pressure and responses to sustained attention in the suprachiasmatic area". Science, 328(5976), 309-309. doi:10.1126/ science. 1177200

Aston-Jones, G., Chiang, C., \& Alexinsky, T. (1991). Discharge of noradrenergic locus coeruleus neurons in behaving rats and monkeys suggests a role in vigilance. Progress in Brain Research, 88(100), 501-520. doi:10.1016/S0079-6123(08) 63830-3 
Aston-Jones, G. \& Cohen, J. D. (2005). An integrative theory of locus coeruleusnorepinephrine function: Adaptive gain and optimal performance. Annual Review of Neuroscience , 28, 403-50. doi:10.1146/annurev.neuro.28.061604.135709

Aston-Jones, G., Rajkowski, J., \& Cohen, J. D. (1999). Role of locus coeruleus in attention and behavioral flexibility. Biological Psychiatry, 46(9), 1309-1320. doi:10.1016/S0006-3223(99)00140-7

Aston-Jones, G., Rajkowski, J., \& Kubiak, P. (1997). Conditioned responses of monkey locus coeruleus neurons anticipate acquisition of discriminative behavior in a vigilance task. Neuroscience, 80(3), 697-715. doi:10.1016/S0306-4522(97) 00060-2

Aston-Jones, G., Rajkowski, J., Kubiak, P., \& Alexinsky, T. (1994). Locus coeruleus neurons in monkey are selectively activated by attended cues in a vigilance task. The Journal of Neuroscience, 14(July), 4467-4480.

Atkinson, R. C. \& Shiffrin, R. M. (1968). Human memory: A proposed system and its control processes. Psychology of Learning and Motivation, 2, 89-195. doi:10. 1016/S0079-7421(08)60422-3

Averbach, E. \& Coriell, A. S. (1961). Short-term memory in vision. Bell System Technical Journal, 40, 309-328. doi:10.1002/j.1538-7305.1961.tb03987.x

Awh, E., Barton, B., \& Vogel, E. K. (2007). Visual working memory representes a fixed number of items regardless of complexity. Psychological Science, 18(7), 622-628. doi:10.1111/j.1467-9280.2007.01949.x

Backs, R. W. \& Walrath, L. C. (1992). Eye-Movement and Pupillary Response Indexes of Mental Workload During Visual-Search of Symbolic Displays. Applied Ergonomics, 23(4), 243-254. doi:10.1016/0003-6870(92)90152-L

Bacon, W. F. \& Egeth, H. E. (1994). Overriding stimulus-driven attentional capture. Perception \& Psychophysics, 55(5), 485-496. doi:10.3758/BF03205306

Baddeley, A. D. (1986). Working memory. Oxford: Oxford University Press.

Baddeley, A. D. (1992). Working memory. Science, 255(5044), 556-559. doi:10.1126/ science. 1736359

Baddeley, A. D. (1996). Exploring the central executive. The Quarterly Journal of Experimental Psychology, 49A(1), 5-28. doi:10.1080/713755608

Baddeley, A. D. (2000). The episodic buffer: A new component of working memory? Trends in Cognitive Sciences, 4(11), 417-423. doi:10.1016/S1364-6613(00) 01538-2 
Baddeley, A. D. (2002). Is working memory still working? European Psychologist, 7(2), 85-97. doi:10.1027//1016-9040.7.2.85

Baddeley, A. D. (2003). Working memory: Looking back and looking forward. Nature Reviews Neuroscience, 4(10), 829-839. doi:10.1038/nrn1201

Baddeley, A. D. (2010). Working memory. Current Biology, 20(4), R136-40. doi:10. 1016/j.cub.2009.12.014

Baddeley, A. D. \& Colquhoun, W. P. (1969). Signal probability and vigilance: A reappraisal of the 'signal-rate' effect. British Journal of Psychology, 60(2), 169178. doi:10.1111/j.2044-8295.1969.tb01189.x

Baddeley, A. D. \& Hitch, G. (1974). Working memory. In The psychology of learning and motivation: Advances in research and theory (Vol. 8, pp. 47-89). doi:10. 1016/S0079-7421(08)60452-1

Baddeley, A. D., Thomson, N., \& Buchanan, M. (1975). Word length and the structure of short-term memory. Journal of Verbal Learning and Verbal Behavior, 14(6), 575-589. doi:10.1016/S0022-5371(75)80045-4

Baker, C. H. (1963). Signal duration as a factor in vigilance tasks. Science, 141(12), 1196-1198. doi:10.1126/science.141.3586.1196

Ballard, J. C. (1996). Computerized assessment of sustained attention: A review of factors affecting vigilance performance. Journal of Clinical and Experimental Neuropsychology, 18(6), 843-863. doi:10.1080/01688639608408307

Bárány, E. H. \& Halldén, U. (1948). Phasic inhibition of the light reflex of the pupil during retinal rivalry. Journal of Neurophysiology, 11(1), 25-30. Retrieved from http://jn.physiology.org/content/11/1/25.full.pdf+html

Barbur, J. L., Harlow, A. J., \& Sahraie, A. (1992). Pupillary responses to stimulus structure, colour and movement. Ophthalmic \& Physiological Optics, 12(2), 137141. doi:10.1111/j.1475-1313.1992.tb00276.x

Barbur, J. L., Wolf, J., \& Lennie, P. (1998). Visual processing levels revealed by response latencies to changes in different visual attributes. Proceedings of the Royal Society of London B, 265(1412), 2321-2325. doi:10.1098/rspb.1998.0578

Basner, M. \& Dinges, D. F. (2011). Maximizing sensitivity of the psychomotor vigilance test (PVT) to sleep loss. Sleep, 34(5), 581-91. doi:10.1093/sleep/34.5.581

Basner, M., Mollicone, D., \& Dinges, D. F. (2011). Validity and sensitivity of a brief psychomotor vigilance test (PVT-B) to total and partial sleep deprivation. Acta Astronautica, 69(11-12), 949-959. doi:10.1016/j.actaastro.2011.07.015 
Bays, P. M. \& Husain, M. (2008). Dynamic shifts of limited working memory resources in human vision. Science, 321(5890), 851-854. doi:10.1126/science.1158023

Beatty, J. (1982a). Phasic not tonic pupillary responses vary with auditory vigilance performance. Psychophysiology, 19(2), 167-172. doi:10.1111/j.1469-8986.1982. tb02540.x

Beatty, J. (1982b). Task-evoked pupillary responses, processing load, and the structure of processing resources. Psychological Bulletin. Retrieved from http://psycnet. apa.org/journals/bul/91/2/276/

Beatty, J. \& Lucero-Wagoner, B. (2000). The pupillary system. In Handbook of psychophysiology. Retrieved from http://sunburst.usd.edu/\%7B \%7Dschieber/docs/ Beatty\%7B\%5C_\%7DPupillary\%7B\%5C_\%7DSystem\%7B\%5C_\%7D.pdf

Beatty, J. \& Wagoner, B. L. (1978). Pupillometric signs of brain activation vary with level of cognitive processing. Science, 199(4334), 1216-1218. Retrieved from http://www.sciencemag.org/content/199/4334/1216.short

Beck, M. R., Lohrenz, M. C., \& Trafton, J. G. (2010). Measuring search efficiency in complex visual search tasks: global and local clutter. Journal of Experimental Psychology: Applied, 16(3), 238-250. doi:10.1037/a0019633

Beck, M. R., Peterson, M. S., Boot, W. R., Vomela, M., \& Kramer, A. F. (2006). Explicit memory for rejected distractors during visual search. Visual Cognition, 14(2), 150-174. doi:10.1080/13506280600574487

Beck, M. R., Peterson, M. S., \& Vomela, M. (2006). Memory for where, but not what, is used during visual search. Journal of Experimental Psychology: Human Perception and Performance, 32(2), 235-250. doi:10.1037/0096-1523.32.2.235

Becker, W. \& Fuchs, A. F. (1985). Prediction in the oculomotor system: Smooth pursuit during transient dissapearance of a visual target. Experimental Brain Research, $57,562-575$.

Bell, C. J., Shenoy, P., Chalodhorn, R., \& Rao, R. P. N. (2008). Control of a humanoid robot by a noninvasive brain-computer interface in humans. Journal of Neural Engineering, 5, 214-220. doi:10.1088/1741-2560/5/2/012

Berbaum, K. S., Franken, E. A., Dorfman, D. D., Rooholamini, S. A., Kathol, M. H., Barloon, T. J., ... El-Khoury, G. Y. (1990). Satisfaction of search in diagnostic radiology. Investigative Radiology, 25(2), 133-140. doi:10.1097/00004424199002000-00006

Bergen, J. R. \& Julesz, B. (1983a). Parallel versus serial processing in rapid pattern discrimination. Nature, 303(5919), 696-8. doi:10.1038/303696a0 
Bergen, J. R. \& Julesz, B. (1983b). Rapid discrimination of visual patterns. IEEE Transactions on Systems, Man \& Cybernetics, SMC-13(5), 857-863. doi:10.1109/ TSMC.1983.6313080

Berger, H. (1929). Uber das elektrenkephalogramm des menshen. Archiv für Psychiatrie and Nervenkrankheiten, 278(1875), 87: 527-570. doi:10.1007/BF01797193

Berridge, C. W. (2008). Noradrenergic modulation of arousal. Brain Research Reviews, 58(1), 1-17. doi:10.1016/j.brainresrev.2007.10.013

Berridge, C. W., Schmeichel, B. E., \& España, R. A. (2012). Noradrenergic modulation of wakefulness/arousal. Sleep Medicine Reviews, 16(2), 187-197. doi:10.1016/j. smrv.2011.12.003

Berridge, C. W. \& Waterhouse, B. D. (2003). The locus coeruleus-noradrenergic system: Modulation of behavioral state and state-dependent cognitive processes. Brain Research Reviews, 42(1), 33-84. doi:10.1016/S0165-0173(03)00143-7

Berrien, F. K. \& Huntington, G. H. (1943). An exploratory study of pupillary responses during deception. Journal of Experimental Psychology, 32(5), 443. doi:10.1037/ h0063488

Bhavsar, P., Srinivasan, B., \& Srinivasan, R. (2016). Pupillometry based real-time monitoring of operator's cognitive workload to prevent human error during abnormal situations. Industrial and Engineering Chemistry Research, 55(12), 3372-3382. doi:10.1021/acs.iecr.5b03685

Biederman, I. (1972). Perceiving real-world scenes. Science, 177, 77-80.

Biederman, I. \& Ju, G. (1988). Surface versus edge-based determinants of visual recognition. Cognitive Psychology, 20(1), 38-64. doi:10.1016/0010-0285(88) 90024-2

Biggs, A. T. (2017). Getting satisfied with "satisfaction of search": How to measure errors during multiple-target visual search. Attention, Perception, \& Psychophysics, 79(5), 1352-1365. doi:10.3758/s13414-017-1300-2

Binda, P. \& Murray, S. O. (2015). Spatial attention increases the pupillary response to light changes. Journal of Vision, 15, 1-13. doi:10.1167/15.2.1.doi

Binda, P., Pereverzeva, M., \& Murray, S. O. (2013a). Attention to bright surfaces enhances the pupillary light reflex. The Journal of Neuroscience, 33(5), 2199204. doi:10.1523/JNEUROSCI.3440-12.2013

Binda, P., Pereverzeva, M., \& Murray, S. O. (2013b). Pupil constrictions to photographs of the sun. Journal of Vision, 13(6), 8-8. doi:10.1167/13.6.8 
Binda, P., Pereverzeva, M., \& Murray, S. O. (2014). Pupil size reflects the focus of feature-based attention. Journal of Neurophysiology, 112(12), 3046-3052. doi:10.1152/jn.00502.2014

Bitsios, P., Prettyman, R., \& Szabadi, E. U. (1996). Changes in autonomic function with age: A study of pupillary kinetics in healthy young and old people. Age and Ageing, 25(6), 432-438. doi:10.1093/ageing/25.6.432

Bitsios, P., Szabadi, E. U., \& Bradshaw, C. M. (2002). Relationship of the 'fear-inhibited light reflex' to the level of state / trait anxiety in healthy subjects. International Journal of Psychophysiology, 43, 177-184.

Bitsios, P., Szabadi, E. U., \& Bradshaw, C. M. (2004). The fear-inhibited light reflex: Importance of the anticipation of an aversive event. International Journal of Psychophysiology, 52, 87-95. doi:10.1016/j.ijpsycho.2003.12.006

Blackwell, P. J. \& Belt, J. A. (1971). Effect of differential levels of ambient noise on vigilance performance. Perceptual and motor skills, 32(3), 734. doi:10.2466/pms. 1971.32.3.734

Blair, R. C. \& Karniski, W. (1993). An alternative method for significance testing of waveform difference potentials. Psychophysiology, 30, 518-524. doi:10.1111/j. 1469-8986.1993.tb02075.x

Blatter, K., Graw, P., Münch, M., Knoblauch, V., Wirz-Justice, A., \& Cajochen, C. (2006). Gender and age differences in psychomotor vigilance performance under differential sleep pressure conditions. Behavioural Brain Research, 168(2), 312317. doi:10.1016/j.bbr.2005.11.018

Bliss, T. V. \& Collingridge, G. L. (1993). A synaptic model of memory: long-term potentiation in the hippocampus. Nature, 361(6407), 31-39. doi:10.1038/361031a0

Bocca, M.-1. \& Denise, P. (2006). Total sleep deprivation effect on disengagement of spatial attention as assessed by saccadic eye movements. Clinical Neurophysiology, 117(117), 894-899. doi:10.1016/j.clinph.2006.01.003

Boehnke, S. E. \& Munoz, D. P. (2008). On the importance of the transient visual response in the superior colliculus. Current Opinion in Neurobiology, 18(6), 544551. doi:10.1016/j.conb.2008.11.004

Boersma, F., Wilton, K., Barham, R., \& Muir, W. (1970). Effects of arithmetic problem difficulty on pupillary dilation in normals and educable retardates. Journal of Experimental Child Psychology, 9, 142-155. 
Bouret, S. \& Richmond, B. J. (2009). Relation of locus coeruleus neurons in monkeys to Pavlovian and operant behaviors. Journal of Neurophysiology, 101(2), 898-911. doi:10.1152/jn.91048.2008

Bouret, S. \& Sara, S. J. (2004). Reward expectation, orientation of attention and locus coeruleus-medial frontal cortex interplay during learning. European Journal of Neuroscience, 20(3), 791-802. doi:10.1111/j.1460-9568.2004.03526.x

Bouret, S. \& Sara, S. J. (2005). Network reset: A simplified overarching theory of locus coeruleus noradrenaline function. Trends in Neurosciences, 28(11), 574-582. doi:10.1016/j.tins.2005.09.002

Braak, H., Thal, D. R., Ghebremedhin, E., \& Tredici, K. D. (2011). Stages of the pathologic process in alzheimer disease: Age categories from 1 to 100 years. Journal of Neuropathology and Experimental Neurology, 70(11), 960-969. doi:10. 1097/NEN.0b013e318232a379

Bradley, M. M., Miccoli, L., Escrig, M. A., \& Lang, P. J. (2008). The pupil as a measure of emotional arousal and autonomic activation. Psychophysiology, 45(4), 602-7. doi:10.1111/j.1469-8986.2008.00654.x

Bradshaw, J. L. (1968). Pupil size and problem solving. The Quarterly Journal of Experimental Psychology, 20(2), 116-22. doi:10.1080/14640746808400139

Braun, J. \& Sagi, D. (1990). Vision outside the focus of attention. Perception \& Psychophysics, 48(1), 45-58. doi:10.3758/BF03205010

Brisson, J., Mainville, M., Mailloux, D., Beaulieu, C., Serres, J., \& Sirois, S. (2013). Pupil diameter measurement errors as a function of gaze direction in corneal reflection eyetrackers. Behavior Research Methods. doi:10.3758/s13428-0130327-0

Broadbent, D. E. (1953). Noise, paced performance and vigilance tasks. British Journal of Psychology, 44(4), 295-303. doi:10.1111/j.2044-8295.1953.tb01210.x

Broadbent, D. E. (1954). Some effects of noise on visual performance. The Quarterly Journal of Experimental Psychology, 6(1), 1-5. doi:10.1080/17470215408416643

Broadbent, D. E. (1958). Perception and communication. Oxford: Oxford University Press.

Broadbent, D. E. (1971). Decision and stress. London: Academic Press.

Broadbent, D. E. \& Broadbent, M. H. (1987). From detection to identification: Response to multiple targets in rapid serial visual presentation. Perception \& Psychophysics, 42(2), 105-113. doi:10.3758/BF03210498 
Broadbent, D. E. \& Gregory, M. (1963). Vigilance considered as a statistical decision. British Journal of Psychology, 54(4), 309-323. doi:10.1111/j.2044-8295.1963. tb00886.x

Broadbent, D. E. \& Gregory, M. (1965). Effects of noise and of signal rate upon vigilance vnalysed by means of decision theory. Human Factors, 7, 155-162. doi:10.1177/001872086500700207

Brookings, J. B., Wilson, G. F., \& Swain, C. R. (1996). Psychophysiological responses to changes in workload during simulated air traffic control. Biological Psychology, 42(3), 361-377. doi:10.1016/0301-0511(95)05167-8

Buck, L. (1966). Reaction time as a measure of perceptual vigilance. Psychological Bulletin, 65(5), 291-304.

Bullmore, E. T., Suckling, J., Overmeyer, S., Rabe-Hesketh, S., Taylor, E., \& Brammer, M. J. (1999). Global, voxel, and cluster tests, by theory and permutation, for a difference between two groups of structural MR images of the brain. IEEE Transactions on Medical Imaging, 18(1), 32-42. doi:10.1109/42.750253

Caggiano, D. M. \& Parasuraman, R. (2004). The role of memory representation in the vigilance decrement. Psychonomic Bulletin \& Review, 11(5), 932-7. Retrieved from http://www.pubmedcentral.nih.gov/articlerender.fcgi?artid=1351035\%7B\% 5C\&\%7Dtool=pmcentrez\%7B\%5C\&\%7Drendertype=abstract

Cain, M. S., Adamo, S. H., Mitroff, S. R., Cosson, P., \& Dash, R. (2013). A taxonomy of errors in multiple-target visual search. Visual Cognition, 21(7), 899-921. doi:10.1080/13506285.2013.843627

Cain, M. S., Vul, E., Clark, K., \& Mitroff, S. R. (2012). An optimal foraging model of human visual search. Psychological Science, 23(9), 1047-1054. doi:10.1177/ 0956797612440460

Caldwell, J. A., Prazinko, B., \& Caldwell, J. L. (2003). Body posture affects electroencephalographic activity and psychomotor vigilance task performance in sleepdeprived subjects. Clinical Neurophysiology, 114(1), 23-31. doi:10.1016/S13882457(02)00283-3

Campbell, F. W. \& Gregory, A. H. (1960). The effect of pupil size on visual acuity. Nature, 187, 1121-1123. doi:10.1038/1871121c0

Card, S. K., Moran, T. P., \& Newell, A. (1983). The psychology of human-computer interaction. doi:10.1007/s13398-014-0173-7.2

Carrasco, M. (2011). Visual attention: The past 25 years. Vision Research, 51(13), 1484-1525. doi:10.1016/j.visres.2011.04.012 
Carrasco, M., Evert, D. L., Chang, I., \& Katz, S. M. (1995). The eccentricity effect: Target eccentricity affects performance on conjunction searches. Perception \& Psychophysics, 57(8), 1241-1261. doi:10.3758/BF03208380

Cavanagh, J. F., Wiecki, T. V., Kochar, A., \& Frank, M. J. (2014). Eye tracking and pupillometry are indicators of dissociable latent decision processes. Journal of Experimental Psychology: General, 143(4), 1476-1488. doi:10.1037/a0035813

Cave, K. R. \& Wolfe, J. M. (1990). Modeling the role of parallel processing in visual search. Cognitive Psychology, 22(2), 225-271. doi:10.1016/0010-0285(90)90017$\mathrm{X}$

Cedarbaum, J. M. \& Aghajanian, G. K. (1978). Afferent projections to the rat locus coeruleus as determined by a retrograde tracing technique. Journal of Comparative Neurology, 178(1), 1-15. doi:10.1002/cne.901780102

Charness, N., Reingold, E. M., Pomplun, M., \& Stampe, D. M. (2001). The perceptual aspect of skilled performance in chess: Evidence from eye movements. Memory \& Cognition, 29(8), 1146-1152. doi:10.3758/BF03206384

Charnov, E. L. (1976). Optimal foraging theory: The marginal value theorem. Theoretical Population Biology, 9, 129-136. doi:10.1016/0040-5809(76)90040-X

Cherry, E. C. (1953). Some experiments on the recognition of speech, with one and with 2 ears. doi:10.1121/1.1907229

Chua, E. C.-P., Tan, W.-Q., Yeo, S.-C., Lau, P., Lee, I., Mien, I. H., .. Gooley, J. J. (2012). Heart rate variability can be used to estimate sleepiness-related decrements in psychomotor vigilance during total sleep deprivation. Sleep, 35(3). doi:10.5665/ sleep. 1688

Chua, K.-P. \& Chun, M. M. (2003). Implicit scene learning is viewpoint dependent. Perception \& Psychophysics, 65(1), 72-80. doi:10.3758/BF03194784

Chun, M. M. (2000). Contextual cueing of visual attention. Trends in Cognitive Sciences, 4(5), 170-178. doi:10.1016/S1364-6613(00)01476-5

Chun, M. M. \& Jiang, Y. (1998). Contextual cueing: Implicit learning and memory of visual context guides spatial attention. Cognitive Psychology, 36(1), 28-71. doi:10.1006/cogp.1998.0681

Chun, M. M. \& Jiang, Y. (1999). Top-down attentional guidance based on implicit learning of visual covariation. Psychological Science, 10(4), 360-365. doi:10. $1111 / 1467-9280.00168$ 
Chun, M. M. \& Potter, M. C. (1995). A two-stage model for multiple target detection in rapid serial visual presentation. Journal of Experimental Psychology: Human Perception and Performance, 21(1), 109-127. doi:10.1037/0096-1523.21.1.109

Clarke, R. J. \& Ikeda, H. (1985). Luminance and darkness detectors in the olivary and posterior pretectal nuclei and their relationship to the pupillary light reflex in the rat. Experimental Brain Research, 57(2), 224-232. doi:10.1007/BF00236527

Clarke, R. J., Zhang, H., \& Gamlin, P. D. R. (2003). Characteristics of the pupillary light reflex in the alert rhesus monkey. Journal of Neurophysiology, 89(6), 3179-3189. doi:10.1152/jn.01131.2002

Clayton, E. C., Rajkowski, J., Cohen, J. D., \& Aston-Jones, G. (2004). Phasic activation of monkey locus ceruleus neurons by simple decisions in a forced-choice task. The Journal of Neuroscience, 24(44), 9914-9920. doi:10.1523/JNEUROSCI.244604.2004

Cohen, A. \& Ivry, R. B. (1991). Density effects in conjunction search: Evidence for a coarse location mechanism of feature integration. Journal of Experimental Psychology: Human Perception and Performance, 17(4), 891-901. doi:10.1037/ 0096-1523.17.4.891

Collet, C., Roure, R., Rada, H., Dittmar, A., \& Vernet-Maury, E. (1996). Relationships between performance and skin resistance evolution involving various motor skills. Physiology and Behavior, 59(4-5), 953-963. doi:10.1016/0031-9384(95)02183-3

Colman, F. D. (1969). Pupillary response and galvanic skin response during an imagery task. Psychonomic Science, 16(6), 296-297. Retrieved from http://download. springer.com/static/pdf/405/art:10.3758/BF03332696.pdf?auth66=1405370908\% 7B\%5C_\%7D\%7B\%5C\&\%7Dext=.pdf

Colquhoun, W. P. (1971). Circadian variations in mental efficiency. In W. P. Colquhoun (Ed.), Biological rhythms and human performance. London: Academic Press.

Colquhoun, W. V. (1961). The effect of 'unwanted' signals on performance in a vigilance task. Ergonomics, 4(1), 41-51. doi:10.1080/00140136108930506

Corbett, E. A. \& Smith, P. L. (2017). The magical number one-on-square-root-two: The double-target detection deficit in brief visual displays. Journal of Experimental Psychology: Human Perception and Performance, 43(7), 1376-1396. doi:10. 1037/xhp0000386

Corbetta, M. \& Shulman, G. L. (2002). Control of goal-directed and stimulus-driven attention in the brain. Nature Reviews Neuroscience, 3(3), 201-15. doi:10.1038/ $\operatorname{nrn} 755$ 
Corneil, B. D. \& Munoz, D. P. (2014). Overt responses during covert orienting. Neuron, 82(6), 1230-1243. doi:10.1016/j.neuron.2014.05.040

Costa, V. D. \& Rudebeck, P. H. (2016). More than meets the eye: The relationship between pupil size and locus coeruleus activity. Neuron, 89(1), 8-10. doi:10. 1016/j.neuron.2015.12.031

Cowan, N. (1999). An embedded-processes model of working memory. In A. Miyake $\&$ P. Shah (Eds.), Models of working memory: Mechanisms of active maintenance and executive control (pp. 62-101). Cambridge: Cambridge University Press. doi:10.1017/S0140525X01003922

Cowan, N. (2008). What are the differences between long-term, short-term, and working memory? In W. S. Sossin, J.-C. Lacaille, V. F. Castelluci, \& S. Belleville (Eds.), Progress in brain research (Chap. 20, Vol. 169, 07, pp. 323-338). New York: Elsevier. doi:10.1016/S0079-6123(07)00020-9

Cowan, N. (2010). The magical mystery four: How is working memory capacity limited, and why? Current Directions in Psychological Science, 19(1), 51-57. doi:10. 1177/0963721409359277

Craig, A. (1985). Field studies of human inspection: The application of vigilance research. In S. Folkard \& T. Monk (Eds.), Hours of work: Temporal factors in work-scheduling (pp. 133-145). Chichester, UK: Wiley.

Craik, F. I. M. \& Lockhart, R. S. (1972). Levels of processing: A framework for memory research. Journal of Verbal Learning and Verbal Behavior, 11(6), 671684. doi:10.1016/S0022-5371(72)80001-X

Crawford, B. H. (1936). The dependence of pupil size upon external light stimulus under static and variable conditions. Proceedings of the Royal Society of London B, 121(823), 376-395. doi:10.1098/rspb.1936.0072

Cunningham, S., Scerbo, M. W., \& Freeman, F. G. (2000). The electrocortical correlates of daydreaming during vigilance tasks. Journal of Mental Imagery, 24(1-2), 6172.

Dadashi, N., Stedmon, A. W., \& Pridmore, T. P. (2013). Semi-automated CCTV surveillance: The effects of system confidence, system accuracy and task complexity on operator vigilance, reliance and workload. Applied Ergonomics, 44(5), 730-738. doi:10.1016/j.apergo.2012.04.012

Danziger, S., Kingstone, A., \& Snyder, J. J. (1998). Inhibition of return to successively stimulated locations in a sequential visual search paradigm. Journal of Exper- 
imental Psychology: Human Perception and Performance, 24(5), 1467-1475. doi:10.1037/0096-1523.24.5.1467

Davenport, W. G. (1972). Vigilance and arousal: Effects of different types of background stimulation. Journal of Psychology: Interdisciplinary and Applied, 82(2), 339346. doi:10.1080/00223980.1972.9923824

Davies, D. R., Jones, D. M., \& Taylor, A. (1984). Selective- and sustained-attention tasks: Individual and group differences. In R. Parasuraman \& D. R. Davies (Eds.), Varieties of attention (pp. 395-447). Orlando: Academic Press.

Davies, D. R. \& Krkovic, A. (1965). Skin-conductance, alpha-activity, and vigilance. The American Journal of Psychology, 78(2), 304-306.

Davies, D. R. \& Parasuraman, R. (1977). Cortical evoked potentials and vigilance: A decision theory analysis. In R. R. Mackie (Ed.), Vigilance: Theory, operational performance and physiological correlates. New York.

De Gennaro, L., Ferrara, M., Urbani, L., \& Bertini, M. (2000). Oculomotor impairment after 1 night of total sleep deprivation: a dissociation between measures of speed and accuracy. Clinical Neurophysiology, 111, 1771-1778. doi:10.1016/S13882457(00)00393-X

de Gee, J. W., Colizoli, O., Kloosterman, N. A., Knapen, T., Nieuwenhuis, S., \& Donner, T. H. (2017). Dynamic modulation of decision biases by brainstem arousal systems. eLife, 6(50), 1-36. doi:10.7554/eLife.23232

de Gee, J. W., Knapen, T., \& Donner, T. H. (2014). Decision-related pupil dilation reflects upcoming choice and individual bias. Proceedings of the National Academy of Sciences, USA, 111(5), E618-25. doi:10.1073/pnas.1317557111

DeCharms, C. R., Maeda, F., Glover, G. H., Ludlow, D., Pauly, J. M., Soneji, D., ... Mackey, S. C. (2005). Control over brain activation and pain learned by using real-time functional MRI. Proceedings of the National Academy of Sciences, USA, 102(51), 18626-18631.

Dember, W. N., Galinsky, T. L., \& Warm, J. S. (1992). The role of choice in vigilance performance. Bulletin of the Psychonomic Society, 30(3), 201-204. doi:10.3758/ BF03330441

Dember, W. N. \& Warm, J. S. (1979). The psychology of perception (2nd). New York: Rinehart and Winstone.

Di Lollo, V., Kawahara, J., Zuvic, S. M., \& Visser, T. A. (2001). The preattentive emperor has no clothes: A dynamic redressing. Journal of Experimental Psychology: General, 130(OCTOBER). doi:10.1037/0096-3445.130.3.479 
Dinges, D. F., Pack, F., Williams, K., Gillen, K. A., Powell, J. W., Ott, G. E., ... Pack, A. I. (1997). Cumulative sleepiness, mood disturbance, and psychomotor vigilance performance decrements during a week of sleep restricted to 4-5 hours per night. Sleep, 20(4), 267-77. Retrieved from http://www.ncbi.nlm.nih.gov/ pubmed/9231952

Dinges, D. F. \& Powell, J. W. (1985). Microcomputer analyses of performance on a portable, simple visual RT task during sustained operations. Behavior Research Methods, Instruments, \& Computers, 17(6), 652-655. doi:10.3758/BF03200977

Dionisio, D. P., Granholm, E., Hillix, W. A., \& Perrine, W. F. (2001). Differentiation of deception using pupillary responses as an index of cognitive processing. Psychophysiology, 38(2), 205-211. doi:10.1017/S0048577201990717

Ditchburn, R. W. \& Ginsborg, B. L. (1952). Vision with a stabilized retinal image. Nature, 170, 36-37. doi:10.1038/170036a0

Donald, F. M. (2008). The classification of vigilance tasks in the real world. Ergonomics, 51(11), 1643-1655. doi:10.1080/00140130802327219

Donald, F. M., Donald, C., \& Thatcher, A. (2015). Work exposure and vigilance decrements in closed circuit television surveillance. Applied Ergonomics, 47(October 2014), 220-228. doi:10.1016/j.apergo.2014.10.001

Donchin, E. (1981). Presidential address, 1980. Surprise!...Surprise? doi:10.1111/j.14698986.1981.tb01815.x

Donovan, T., Manning, D. J., \& Crawford, T. J. (2008). Performance changes in lung nodule detection following perceptual feedback of eye movements. In Proceedings of spie (Vol. 6917, 1, pp. 1-9). doi:10.1117/12.768503

Donovan, T., Manning, D. J., Phillips, P. W., Higham, S., \& Crawford, T. J. (2005). The efect of feedback on performance in a fracture detection task. In Proceedings of spie (Vol. 5749, April, pp. 79-85). doi:10.1117/12.593294

Dorrian, J., Lamond, N., Holmes, A. L., Burgess, H. J., Roach, G. D., Fletcher, A., \& Dawson, D. (2003). The ability to self-monitor performance during a week of simulated night shifts. Sleep, 26(7), 871-7.

Dorrian, J., Roach, G. D., Fletcher, A., \& Dawson, D. (2007). Simulated train driving: Fatigue, self-awareness and cognitive disengagement. Applied Ergonomics, 38(2), 155-166. doi:10.1016/j.apergo.2006.03.006

Dorrian, J., Rogers, N. L., \& Dinges, D. F. (2005). Psychomotor vigilance performance: Neurocognitive assay sensitive to sleep loss. Sleep deprivation: Clinical issues, pharmacology and sleep loss effects, 39-70. 
Downing, C. J. \& Pinker, S. (1985). The spatial structure of visual attention. In Attention and performance $x i$ (pp. 171-188).

Drummond, S. P. A., Bischoff-Grethe, A., Dinges, D. F., Ayalon, L., Mednick, S. C., \& Meloy, M. J. (2005). The neural basis of the psychomotor vigilance task. Sleep, 28(9), 1059-1068.

Duchowski, A. T. (2002). A breadth-first survey of eye-tracking applications. Behavior Research Methods, Instruments, \& Computers, 34(4), 455-470. doi:10.3758/ BF03195475

Duchowski, A. T. (2007). Eye tracking methodology: Theory and practice (2nd). London: Springer. doi:10.1007/978-1-84628-609-4

Duchowski, A. T., Medlin, E., Gramopadhye, A., Melloy, B., \& Nair, S. (2001). Binocular eye tracking in VR for visual inspection training. Proceedings of the ACM symposium on Virtual reality software and technology - VRST '01, 1. doi:10.1145/ 505009.505010

Duncan, J. (1980). The locus of interference in the perception of simultaneous stimuli. Psychological Review, 87(3), 272-300. doi:10.1037/0033-295X.87.3.272

Duncan, J. \& Humphreys, G. W. (1989). Visual search and stimulus similarity. Psychological Review, 96(3), 433-58. Retrieved from http://www.ncbi.nlm.nih.gov/ pubmed/2756067

Duncan, J. \& Humphreys, G. W. (1992). Beyond the search surface - visual-search and attentional engagement. Journal of Experimental Psychology: Human Perception and Performance, 18(2), 578-588. doi:10.1037/0096-1523.18.2.578

Eckstein, M. P. (2017). Probabilistic computations for attention, eye movements, and search. Annual Review of Vision Science, 3(1), annurev-vision-102016-061220. doi:10.1146/annurev-vision-102016-061220

Eckstein, M. P., Thomas, J. P., Palmer, J., \& Shimozaki, S. S. (2000). A signal detection model predicts the effects of set size on visual search accuracy for feature, conjunction, triple conjunction, and disjunction displays. Perception \& Psychophysics, 62(3), 425-451. doi:10.3758/BF03212096

Ehinger, K. A. \& Wolfe, J. M. (2016). When is it time to move to the next map? Optimal foraging in guided visual search. Attention, Perception, \& Psychophysics, 78(7), 2135-2151. doi:10.1167/13.3.10

Ehlers, J., Bubalo, N., Loose, M., \& Huckauf, A. (2015). Towards voluntary pupil control - training affective strategies? In Proceedings of the 2nd international conference on physiological computing systems (pp. 5-12). doi:10.5220/0005240000050012 
Ehlers, J., Georgi, J., \& Huckauf, A. (2014). Improving voluntary pupil size changes for HCI. Proceedings of the 8th International Conference on Pervasive Computing Technologies for Healthcare, 15-18. doi:10.4108/icst.pervasivehealth.2014. 255312

Ehlers, J., Strauch, C., Georgi, J., \& Huckauf, A. (2016). Pupil size changes as an active information channel for biofeedback applications. Applied Psychophysiology Biofeedback, 41(3), 331-339. doi:10.1007/s10484-016-9335-z

Eimer, M. (2014). The neural basis of attentional control in visual search. Trends in Cognitive Sciences, 1-10. doi:10.1016/j.tics.2014.05.005

Einhäuser, W. (2017). The pupil as a marker of cognitive processes. In Q. Zhao (Ed.), Computational and cognitive neuroscience of vision (pp. 141-169). Singapore: Springer.

Einhäuser, W., Koch, C., \& Carter, O. L. (2010). Pupil dilation betrays the timing of decisions. Frontiers in Human Neuroscience, 4(February), 18. doi:10.3389/fnhum. 2010.00018

Einhäuser, W., Stout, J., Koch, C., \& Carter, O. L. (2008). Pupil dilation reflects perceptual selection and predicts subsequent stability in perceptual rivalry. Proceedings of the National Academy of Sciences, USA, 105(5), 1704-1709. doi:10.1073/pnas. 0707727105

Ekman, I., Poikola, A., Mäkäräinen, M., Takala, T., \& Hämäläinen, P. (2008). Voluntary pupil size change as control in eyes only interaction. In Proceedings of the 2008 symposium on eye-tracking research \& applications (pp. 115-118). New York: ACM. doi:10.1145/1344471.1344501

Ellis, C. J. (1981). The pupillary light reflex in normal subjects. The British Journal of Ophthalmology, 65(11), 754-9. Retrieved from http://www.pubmedcentral.nih. gov/articlerender.fcgi?artid=1039657\%7B\%5C\&\%7Dtool=pmcentrez\%7B\% 5C\&\%7Drendertype $=$ abstract

Enander, A. (1987). Effects of moderate cold on performance of psychomotor and cognitive tasks. Ergonomics, 30(10), 1431-1445. doi:10.1080/00140138708966037

Engbert, R. \& Kliegl, R. (2003). Microsaccades uncover the orientation of covert attention. Vision Research, 43(9), 1035-1045. doi:10.1016/S0042-6989(03) 00084-1

Engbert, R. \& Mergenthaler, K. (2006). Microsaccades are triggered by low retinal image slip. Proceedings of the National Academy of Sciences, USA, 103(18), 7192-7. doi:10.1073/pnas.0509557103 
Engle, R. W., Kane, M. J., \& Tuholski, S. W. (1999). Individual differences in working memory capacity and what they tell us about controlled attention, general fluid intelligence, and functions of the prefrontal cortex. In Models of working memory: Mechanisms of active maintenance and executive control (pp. 102-134). New York: Cambridge University Press.

Englund, M. P. \& Patching, G. R. (2009). An inexpensive and accurate method of measuring the force of responses in reaction time research. Behavior Research Methods, 41(4), 1254-61. doi:10.3758/BRM.41.4.1254

Eriksen, C. W. \& Hoffman, J. E. (1973). The extent of processing of noise elements during selective encoding from visual displays. doi:10.3758/BF03198630

Eriksen, C. W. \& James, D. S. J. (1986). Visual attention within and around the field of focal attention: a zoom lens model. Perception \& Psychophysics, 40(4), 225-240.

Eriksen, C. W. \& Spencer, T. (1969). Rate of information processing in visual perception: Some results and methodological considerations. Journal of Experimental Psychology, 79(2), 1-16. doi:10.1037/h0026873

Esterman, M., Grosso, M., Liu, G., Mitko, A., Morris, R., \& DeGutis, J. (2016). Anticipation of monetary reward can attenuate the vigilance decrement. PLOS ONE, 11(7), e0159741. doi:10.1371/journal.pone.0159741

Esterman, M., Reagan, A., Liu, G., Turner, C., DeGutis, J., Esterman, M., ... Degutis, J. (2014). Reward reveals dissociable aspects of sustained attention. Journal of Experimental Psychology: General, October 13. doi:10.1037/xge0000019

Evans, K. K., Greene, M. R., Wolfe, J. M., \& Võ, M. L.-H. (2011). Visual search in scenes involves selective and nonselective pathways. Trends in Cognitive Sciences, 15(2), 77-84. doi:10.1016/j.tics.2010.12.001

Eysenck, M. W. (2012). Fundamentals of cognition. New York: Psychology Press.

Fabiani, G. E., McFarland, D. J., Wolpaw, J. R., \& Pfurtscheller, G. (2004). Conversion of EEG activity into cursor movement by a brain-computer interface (BCI). IEEE Transactions on Neural Systems \& Rehabilitation Engineering, 12(3), 331-338. doi:10.1109/TNSRE.2004.834627

Fahle, M. W., Stemmler, T., \& Spang, K. M. (2011). How much of the "unconscious" is just pre-threshold? Frontiers in Human Neuroscience, 5(October), 1-6. doi:10. 3389/fnhum.2011.00120

Fan, J., McCandliss, B. D., Fossella, J., Flombaum, J. I., \& Posner, M. I. (2005). The activation of attentional networks. NeuroImage, 26(2), 471-479. doi:10.1016/j. neuroimage.2005.02.004 
Fan, J., McCandliss, B. D., Sommer, T., Raz, A., \& Posner, M. I. (2002). Testing the efficiency and independence of attentional networks. Journal of Cognitive Neuroscience, 14(3), 340-7. doi:10.1162/089892902317361886

Fecteau, J. H. \& Munoz, D. P. (2006). Salience, relevance, and firing: A priority map for target selection. Trends in Cognitive Sciences, 10(8), 382-390. doi:10.1016/j. tics.2006.06.011

Findlay, J. M. (1997). Saccade target selection during visual search. Vision Research, 37(5), 617-31. Retrieved from http://www.ncbi.nlm.nih.gov/pubmed/14765969

Findlay, J. M. \& Gilchrist, I. D. (2001). Visual attention: The active vision perspective. Vision and Attention, 83-104. doi:10.1007/978-0-387-21591-4_5

Findlay, J. M. \& Gilchrist, I. D. (2003). Active vision: The psychology of looking and seeing. New York: Oxford University Press.

Findlay, J. M. \& Gilchrist, I. D. (2005). Eye guidance and visual search, 1-22. doi:10. 1016/B978-008043361-5/50014-6

Findlay, J. M. \& Walker, R. (1999). A model of saccade generation based on parallel processing and competitive inhibition. Behavioral and Brain Sciences, 22(4), 661-721. doi:0140-525X/99

Fleck, M. S., Samei, E., \& Mitroff, S. R. (2010). Generalized "satisfaction of search": Adverse influences on dual-target search accuracy. Journal of Experimental Psychology: Applied, 16(1), 60-71. doi:10.1037/a0018629

Folk, C. L., Remington, R. W., \& Johnston, J. C. (1992). Involuntary covert orienting is contingent on attentional control settings. Journal of Experimental Psychology: Human Perception and Performance, 18(4), 1030-1044. doi:10.1037//00961523.18.4.1030

Folk, C. L., Remington, R. W., \& Wright, J. H. (1994). The structure of attentional control: Contingent attentional capture by apparent motion, abrupt onset, and color. Journal of Experimental Psychology: Human Perception and Performance, 20(2), 317-329. doi:10.1037/0096-1523.20.2.317

Fortenbaugh, F. C., DeGutis, J., \& Esterman, M. (2017). Recent theoretical, neural, and clinical advances in sustained attention research. Annals of the New York Academy of Sciences, 1396, 70-91. doi:10.1111/nyas.13318

Franklin, M. S., Broadway, J. M., Mrazek, M. D., Smallwood, J., \& Schooler, J. W. (2013). Window to the wandering mind: Pupillometry of spontaneous thought while reading. The Quarterly Journal of Experimental Psychology, 66(12), 22892294. doi:10.1080/17470218.2013.858170 
Frankmann, J. P. \& Adams, J. A. (1962). Theories of vigilance. Psychological Bulletin, 59(4), 257-272. doi:10.1037/h0046142

Furdea, A., Halder, S., Krusienski, D. J., Bross, D., Nijboer, F., \& Birbaumer, N. (2009). An auditory oddball (P300) spelling system for brain-computer interfaces. Psychophysiology, 46, 617-625. doi:10.1111/j.1469-8986.2008.00783.x

Gagl, B., Hawelka, S., \& Hutzler, F. (2011). Systematic influence of gaze position on pupil size measurement: Analysis and correction. Behavior Research Methods, 43(4), 1171-81. doi:10.3758/s13428-011-0109-5

Gale, A. (1977). Some EEG correlates of sustained attention. In R. R. Mackie (Ed.), Vigilance: Theory, operational performance and physiological correlates. New York: Plenum.

Galinsky, T. L., Warm, J. S., \& Dember, W. N. (1993). Psychophysical determinants of stress in sustained attention. Human Factors, 35(4), 603-614.

Galley, N. (1989). Saccadic eye movement velocity as an indicator of (de) activation. A review and some speculations. Journal of Psychophysiology, 3, 229-224.

Gandhi, N. J. \& Katnani, H. A. (2011). Motor functions of the superior colliculus. Annual Review of Neuroscience, 34, 205-231. doi:10.1146/annurev - neuro061010-113728

Gandhi, S. P., Heeger, D. J., \& Boynton, G. M. (1999). Spatial attention affects brain activity in human primary. Proceedings of the National Academy of Sciences, USA, 96(March), 3314-3319.

Geisler, W. S. \& Chou, K. L. (1995). Separation of low-level and high-level factors in complex tasks: Visual search. Psychological Review, 102(2), 356-378. doi:10. 1037/0033-295X.102.2.356

Geyer, T., Von Mühlenen, A., \& Müller, H. J. (2007). What do eye movements reveal about the role of memory in visual search? The Quarterly Journal of Experimental Psychology, 60(7), 924-935. doi:10.1080/17470210600831119

Gibson, B. S. \& Kelsey, E. M. (1998). Stimulus-driven attentional capture is contingent on attentional set for displaywide visual features. Journal of Experimental Psychology: Human Perception and Performance, 24(3), 699-706. doi:10.1037/00961523.24.3.699

Gil, Z., Connors, B. W., \& Amitai, Y. (1997). Differential regulation of neocortical synapses by neuromodulators and activity. Neuron, 19(3), 679-686. doi:10.1016/ S0896-6273(00)80380-3 
Gilchrist, I. D. (2011). Saccades. In I. D. Gilchrist, S. P. Liversedge, \& S. Everling (Eds.), The oxford handbook of eye movements (pp. 85-94). Oxford: Oxford University Press.

Gilchrist, I. D. \& Harvey, M. (2000). Refixation frequency and memory mechanisms in visual search. Current Biology, 10(19), 1209-12. Retrieved from http://www. ncbi.nlm.nih.gov/pubmed/11050390

Gill, G. W. (1996). Vigilance in cytoscreening: looking without seeing. Advance for Medical Laboratory Professionals, 8, 14-15.

Gilzenrat, M. S., Nieuwenhuis, S., Jepma, M., \& Cohen, J. D. (2010). Pupil diameter tracks changes in control state predicted by the adaptive gain theory of locus coeruleus function. Cognitive, Affective \& Behavioral Neuroscience, 10(2), 25269. doi:10.3758/CABN.10.2.252

Godwin, H. J., Menneer, T., Cave, K. R., \& Donnelly, N. (2010). Dual-target search for high and low prevalence X-ray threat targets. Visual Cognition, 18(10), 14391463. doi:10.1080/13506285.2010.500605

Godwin, H. J., Menneer, T., Cave, K. R., Helman, S., Way, R. L., \& Donnelly, N. (2010). The impact of Relative Prevalence on dual-target search for threat items from airport X-ray screening. Acta Psychologica, 134(1), 79-84. doi:10.1016/j.actpsy. 2009.12.009

Godwin, H. J., Menneer, T., Cave, K. R., Thaibsyah, M., \& Donnelly, N. (2014). The effects of increasing target prevalence on information processing during visual search. Psychonomic Bulletin \& Review. doi:10.3758/s13423-014-0686-2

Godwin, H. J., Menneer, T., Riggs, C. A., Cave, K. R., \& Donnelly, N. (2014). Perceptual failures in the selection and identification of low-prevalence targets in relative prevalence visual search. Attention, Perception, \& Psychophysics. doi:10.3758/ s13414-014-0762-8

Goldinger, S. D. \& Papesh, M. H. (2012). Pupil dilation reflects the creation and retrieval of memories. Current Directions in Psychological Science, 21(2), 90-95. doi:10.1177/0963721412436811

Goldwater, B. C. (1972). Psychological significance of pupillary movements. Psychological Bulletin, 77(5), 340-355. Retrieved from http://psycnet.apa.org/journals/ bul/77/5/340/

Gorbunova, E. S. (2017). Perceptual similarity in visual search for multiple targets. Acta Psychologica, 173, 46-54. doi:10.1016/j.actpsy.2016.11.010 
Gramfort, A., Luessi, M., Larson, E., Engemann, D. A., Strohmeier, D., Brodbeck, C., .. Hämäläinen, M. S. (2013). MEG and EEG data analysis with MNE-Python. Frontiers in Neuroscience, 7(7 DEC), 1-13. doi:10.3389/fnins.2013.00267

Gramfort, A., Luessi, M., Larson, E., Engemann, D. A., Strohmeier, D., Brodbeck, C., .. Hämäläinen, M. S. (2014). MNE software for processing MEG and EEG data. NeuroImage, 86, 446-460. doi:10.1016/j.neuroimage.2013.10.027

Grandchamp, R., Braboszcz, C., \& Delorme, A. (2014). Oculometric variations during mind wandering. Frontiers in Psychology, 5(FEB), 1-10. doi:10.3389/fpsyg.2014. 00031

Graw, P., Kräuchi, K., Knoblauch, V., Wirz-Justice, A., \& Cajochen, C. (2004). Circadian and wake-dependent modulation of fastest and slowest reaction times during the psychomotor vigilance task. Physiology and Behavior, 80(5), 695-701. doi:10.1016/j.physbeh.2003.12.004

Green, D. M. \& Swets, J. A. (1974). Signal detection theory and psychophysics. Huntington, NY: RF Krieger.

Grier, R. A., Warm, J. S., Dember, W. N., Matthews, G., Galinsky, T. L., \& Parasuraman, R. (2003). The vigilance decrement reflects limitations in effortful attention, not mindlessness. Human Factors, 45(3), 349-59. Retrieved from http://www.ncbi. nlm.nih.gov/pubmed/14702988

Grill-Spector, K., Henson, R., \& Martin, A. (2006). Repetition and the brain: Neural models of stimulus-specific effects. Trends in Cognitive Sciences, 10(1), 14-23. doi:10.1016/j.tics.2005.11.006

Gunzelmann, G., Gross, J. B., Gluck, K. A., \& Dinges, D. F. (2009). Sleep deprivation and sustained attention performance: Integrating mathematical and cognitive modeling. Cognitive Science, 33(5), 880-910. doi:10.1111/j.1551-6709.2009. 01032.x

Gur, D., Sumkin, J. H., Rockette, H. E., Ganott, M., Hardesty, L., Poller, W. R., ... Wallace, L. (2004). Changes in breast cancer detection and mammography recall rates after introduction of a computer aided detection system. Journal of the National Cancer Institute, 96(3), 185-190. doi:10.1093/jnci/djh067

Guthrie, D. \& Buchwald, J. S. (1991). Significance testing of difference potentials. Psychophysiology, 28(2), 240-244. doi:10.1111/j.1469-8986.1991.tb00417.x

Hagger, M. S., Wood, C., Stiff, C., \& Chatzisarantis, N. L. D. (2010). Ego depletion and the strength model of self-control: A meta-analysis. Psychological Bulletin, 136(4), 495-525. doi:10.1037/a0019486 
Hakerem, G. \& Sutton, S. (1966). Pupillary response at visual threshold. Nature, 212(5061), 485-486. doi:10.1038/212485a0

Hancock, P. A. (1984). Environmental stressors. In J. S. Warm (Ed.), Sustained attention in human performance (pp. 1-14). New York: Wiley.

Hancock, P. A. (2013). In search of vigilance: The problem of iatrogenically created psychological phenomena. American Psychologist, 68(2), 97-109. doi:10.1037/ a0030214

Hancock, P. A. \& Hart, S. G. (2002). Defeating terrorism: What can human factors / ergonomics offer? Ergonomics in Design.

Hartley, L. R., Arnold, P. K., Kobryn, H., \& MacLeod, C. (1989). Vigilance, visual search and attention in an agricultural task. Applied Ergonomics, 20(1), 9-16. doi:10.1016/0003-6870(89)90003-3

Hasselmo, M. E. (1999). Neuromodulation: acetylcholine and memory consolidation. Trends in Cognitive Sciences, 3(9), 351-359. doi:10.1016/s1364-6613(99)013650

Hatfield, J. L. \& Loeb, M. (1968). Sense mode and coupling in a vigilance task. Perception \& Psychophysics, 4(1), 29-36. doi:10.3758/BF03210443

Hauty, G. T. \& Payne, R. B. (1955). Mitigation of work decrement. Journal of Experimental Psychology, 49(1), 60-67. doi:10.1037/h0046488

Hawkes, G. R. \& Loeb, M. (1962). Vigilance for cutaneous and auditory stimuli as a function of intersignal interval and signal strength. The Journal of Psychology, 53, 211-218. doi:10.1080/00223980.1962.9916566

Hayes, T. R. \& Petrov, A. A. (2015). Mapping and correcting the influence of gaze position on pupil size measurements. Behavior Research Methods. doi:10.3758/ s13428-015-0588-x

Hayhoe, M. M. (2000). Vision using routines: A functional account of vision. Visual Cognition, 7(1997), 43-64. doi:10.1080/135062800394676

Head, J. \& Helton, W. S. (2012). Natural scene stimuli and lapses of sustained attention. Consciousness and Cognition, 21(4), 1617-1625. doi:10.1016/j.concog.2012.08. 009

Heaver, B. \& Hutton, S. B. (2011). Keeping an eye on the truth? Pupil size changes associated with recognition memory. Memory, 19(4), 398-405. doi:10.1080/ 09658211.2011 .575788 
Heilveil, I. (1976). Deception and pupil size. Journal of Clinical Psychology, 32(3), 675-676. doi:10.1002/1097-4679(197607)32:3<675::AID-JCLP2270320340> 3.0.CO;2-A

Helton, W. S. \& Russell, P. N. (2011). Working memory load and the vigilance decrement. Experimental Brain Research, 212(3), 429-437. doi:10.1007/s00221-0112749-1

Helton, W. S. \& Russell, P. N. (2013). Visuospatial and verbal working memory load: Effects on visuospatial vigilance. Experimental Brain Research, 224(3), 429-436. doi:10.1007/s00221-012-3322-2

Helton, W. S. \& Warm, J. S. (2008). Signal salience and the mindlessness theory of vigilance. Acta Psychologica, 129(1), 18-25. doi:10.1016/j.actpsy.2008.04.002

Hess, E. H. \& Polt, J. M. (1960). Pupil size as related to interest value of visual stimuli. Science, 132(3423), 349-350. Retrieved from http://psycnet.apa.org/psycinfo/ 1961-01581-001

Hess, E. H. \& Polt, J. M. (1964). Pupil size in relation to mental activity during simple problem-solving. Science, 143(3611), 1190-1192. Retrieved from http: //www.sciencemag.org/content/143/3611/1190.short

Hess, E. H., Seltzer, A. L., \& Shlien, J. M. (1965). Pupil response of hetero- and homosexual males to pictures of men and women: A pilot study. Journal of Abnormal Psychology, 70(3), 165-168. doi:10.1037/h0021978

Hirata, Y., Yamaji, K., Sakai, H., \& Usui, S. (2003). Function of the pupil in vision and information capacity of retinal image. Systems and Computers in Japan, 34(9), 48-57. doi:10.1002/scj.10344

Hobson, J. A., McCarley, R. W., \& Wyzinski, P. W. (1975). Sleep cycle oscillation: Reciprocal discharge by two brainstem neuronal groups. Science, 189(4196), 55-58. doi:10.1126/science.1094539

Hodsoll, J. \& Humphreys, G. W. (2001). Driving attention with the top down: The relative contribution of target templates to the linear separability effect in the size dimension. Perception \& Psychophysics, 63(5), 918-926. doi:10.3758/ BF03194447

Hoeks, B. \& Levelt, W. J. M. (1993). Pupillary dilation as a measure of attention: A quantitative system analysis. Behavior Research Methods, Instruments, \& Computers, 25(1), 16-26.

Hofer, F. \& Schwaninger, A. (2005). Using threat image projection data for assessing individual screener performance. WIT Transactions on the Built Environment, 82, 
417-426. Retrieved from http://kyb.tuebingen.mpg.de/publications/attachments/ HoferSchwaninger2005\%7B\%5C_\%7D[0].pdf

Hollands, M., Patla, A. E., \& Vickers, J. N. (2002). "Look where you're going!": Gaze behaviour associated with maintaining and changing the direction of locomotion. Experimental Brain Research, 143(2), 221-230. doi:10.1007/s00221-001-0983-7

Holmes, A. P., Blair, R. C., Watson, J. D. G., \& Ford, I. (1996). Nonparametric analysis of statistic images from functional mapping experiments. Journal of Cerebral Blood Flow \& Metabolism, 16(1), 7-22. doi:10.1097/00004647-19960100000002

Holmqvist, K., Nyström, M., Andersson, R., Dewhurst, R., Jarodzka, H., \& van de Weijer, J. (2011). Eye tracking: A comprehensive guide to methods and measures. Oxford: OUP.

Hong, L., Walz, J. M., \& Sajda, P. (2014). Your eyes give you away: Prestimulus changes in pupil diameter correlate with poststimulus task-related EEG dynamics. PLoS ONE, 9(3), e91321. doi:10.1371/journal.pone.0091321

Honts, C. R., Devitt, M. K., Winbush, M., \& Kircher, J. C. (1996). Mental and physical countermeasures reduce the accuracy of the concealed knowledge test. Psychophysiology, 79(2), 252-259. doi:10.1111/j.1469-8986.1996.tb02111.x

Hooge, I. T. \& Erkelens, C. J. (1996). Control of fixation duration in a simple search task. Perception \& Psychophysics, 58(7), 969-976. doi:10.3758/BF03206825

Hopstaken, J. F., Van der Linden, D., Bakker, A. B., \& Kompier, M. A. J. (2015). A multifaceted investigation of the link between mental fatigue and task disengagement. Psychophysiology, 52(3), 305-315. doi:10.1111/psyp.12339

Hopstaken, J. F., Van der Linden, D., Bakker, A. B., Kompier, M. A. J., \& Leung, Y. K. (2016). Shifts in attention during mental fatigue: Evidence from subjective, behavioral, physiological, and eye-tracking data. Journal of Experimental Psychology: Human Perception and Performance, 42(6), 878-889. doi:10.1037/xhp0000189

Hopstaken, J. F., van der Linden, D., Bakker, A. B., \& Kompier, M. A. J. (2015). The window of my eyes: Task disengagement and mental fatigue covary with pupil dynamics. Biological Psychology, 110, 100-106. doi:10.1016/j.biopsycho.2015. 06.013

Horne, J. A., Brass, C. G., \& Pettitt, A. N. (1980). Ciradian performance differences between morning and evening "types". Ergonomics, 23(January), 29-36. doi:10. 1080/00140138008924715 
Horowitz, T. S. \& Wolfe, J. M. (1998). Visual search has no memory. Nature, 394(6693), 575-577. doi:10.1038/29068

Horowitz, T. S. \& Wolfe, J. M. (2001). Search for multiple targets: Remember the targets, forget the search. Perception \& Psychophysics, 63(2), 272-285. doi:10. 3758/BF03194468

Horowitz, T. S. \& Wolfe, J. M. (2003). Memory for rejected distractors in visual search? Visual Cognition, 10(3), 257-298. doi:10.1080/13506280143000005

Hou, R. H., Freeman, C., Langley, R. W., Szabadi, E. U., \& Bradshaw, C. M. (2005). Does modafinil activate the locus coeruleus in man? Comparison of modafinil and clonidine on arousal and autonomic functions in human volunteers. Psychopharmacology, 181(3), 537-549. doi:10.1007/s00213-005-0013-8

Howell, W. C. (1993). Engineering psychology in a changing world. Annual Review of Psychology, 44, 231-263. doi:10.1146/annurev.psych.44.1.231

Howells, F. M., Stein, D. J., \& Russell, V. A. (2010). Perceived mental effort correlates with changes in tonic arousal during attentional tasks. Behavioral and Brain Functions, 6(39), 1-15. doi:10.1186/1744-9081-6-39

Hsieh, C. Y., Cruikshank, S. J., \& Metherate, R. (2000). Differential modulation of auditory thalamocortical and intracortical synaptic transmission by cholinergic agonist. Brain Research, 880(1-2), 51-64. doi:10.1016/S0006-8993(00)02766-9

Hulleman, J. \& Olivers, C. N. L. (2017). The impending demise of the item in visual search. Behavioral and Brain Sciences, 1-69. doi:10.1017/S0140525X15002794

Hupé, J.-M., Lamirel, C., \& Lorenceau, J. (2009). Pupil dynamics during bistable motion perception. Journal of Vision, 9(7), 10. doi:10.1167/9.7.10

Hyönä, J., Tommola, J., \& Alaja, A. M. (1995). Pupil dilation as a measure of processing load in simultaneous interpretation and other language tasks. The Quarterly Journal of Experimental Psychology, 48A(3), 598-612. Retrieved from http: //www.ncbi.nlm.nih.gov/pubmed/7568993

Inhoff, A. W. (1991). Word frequency during copytyping. Journal of Experimental Psychology: Human Perception and Performance, 17(2), 478-87. doi:10.1037/ 0096-1523.17.2.478

Inhoff, A. W. \& Gordon, A. M. (1997). Eye movements and eye-hand coordination during typing. Current Directions in Psychological Science, 6(6), 153-157. doi:10. 1111/1467-8721.ep10772929 
Itti, L. \& Koch, C. (2000). A saliency-based search mechanism for overt and covert shifts of visual attention. Vision Research, 40(10-12), 1489-506. Retrieved from http://www.ncbi.nlm.nih.gov/pubmed/10788654

Itti, L., Koch, C., \& Niebur, E. (1998). A model of saliency-based visual attention for rapid scene analysis. IEEE Transactions on Pattern Analysis \& Machine Intelligence, 20(11), 1254-1259. Retrieved from http://ieeexplore.ieee.org/xpls/ abs\%7B\%5C_\%7Dall.jsp?arnumber=730558

Jacob, R. J. K. (1991). The use of eye movements in human-computer interaction techniques: what you look at is what you get. ACM Transactions on Information Systems (TOIS), 9(3), 152-169. doi:10.1145/123078.128728

James, W. (1890). The principles of psychology. New York: Holt.

Janisse, M. P. \& Bradley, M. T. (1980). Deception, information and the pupillary response. Perceptual and Motor Skills, 50, 748-750. doi:http://dx.doi.org/10. 2466/pms.1980.50.3.748

Jay, B. S. (1962). The effective pupillary area at varying perimetric angles. Vision Research, 1(418-424). doi:10.1016/0042-6989(62)90021-4

Jepma, M. \& Nieuwenhuis, S. (2011). Pupil diameter predicts changes in the explorationexploitation trade-off: Evidence for the adaptive gain theory. Journal of Cognitive Neuroscience, 23(7), 1587-96. doi:10.1162/jocn.2010.21548

Jerison, H. J. (1959). Effects of noise on human performance. Journal of Applied Psychology, 43(2), 96-101. doi:10.1037/h0042914

Jessen, S., Altvater-Mackensen, N., \& Grossmann, T. (2016). Pupillary responses reveal infants' discrimination of facial emotions independent of conscious perception. Cognition, 150, 163-169. doi:10.1016/j.cognition.2016.02.010

Johnson, D. A. (1971). Pupillary responses during a short-term memory task: Cognitive processing, arousal, or both? Journal of Experimental Psychology, 90(2), 311318. doi:10.1037/h0031562

Johnson, R. F. \& Merullo, D. J. (2000). Caffeine, gender, and sentry duty: Effects of a mild stimulant on vigilance and marksmanship. In K. Friedl, H. Lieberman, D. H. Ryan, \& G. A. Bray (Eds.), Countermeasures for battlefield sressors (pp. 272289). Baton Rouge: Louisiana State University Press.

Johnston, S. J., Boehm, S. G., Healy, D., Goebel, R., \& Linden, D. E. J. (2010). Neurofeedback: A promising tool for the self-regulation of emotion networks. NeuroImage, 49(1), 1066-72. doi:10.1016/j.neuroimage.2009.07.056 
Johnston, S. J., Linden, D. E. J., Healy, D., Goebel, R., Habes, I., \& Boehm, S. G. (2011). Upregulation of emotion areas through neurofeedback with a focus on positive mood. Cognitive, Affective \& Behavioral Neuroscience, 11(1), 44-51. doi:10.3758/s13415-010-0010-1

Johnston, W. A. \& Dark, V. J. (1986). Selective attention. Annual Review of Psychology, $37,43-75$.

Jolicoeur, P., Dell'Acqua, R., \& Crebolder, J. (2001). The attentional blink bottleneck. In K. L. Shapiro (Ed.), The limits of attention (pp. 82-99). Oxford: Oxford University Press. doi:10.1093/acprof:oso/9780198505150.003.0005

Jonides, J. (1981). Voluntary versus automatic control over the mind's eye's movement. doi:10.1037/0096-1523.29.5.835

Joshi, S., Li, Y., Kalwani, R. M., \& Gold, J. I. (2016). Relationships between pupil diameter and neuronal activity in the locus coeruleus, colliculi, and cingulate cortex. Neuron, 89(1), 221-234. doi:10.1016/j.neuron.2015.11.028

Kafkas, A. \& Montaldi, D. (2011). Recognition memory strength is predicted by pupillary responses at encoding while fixation patterns distinguish recollection from familiarity. The Quarterly Journal of Experimental Psychology, 64(10), 1971-1989. doi:10.1080/17470218.2011.588335

Kahneman, D. (1973). Attention and effort. Englewood Cliffs, NJ: Prentice-Hall. doi:10. $2307 / 1421603$

Kahneman, D. \& Beatty, J. (1966). Pupil diameter and load on memory. Science, 154(3756), 1583-1585. Retrieved from http://doi.apa.org/psycinfo/1967-02420001

Kahneman, D. \& Beatty, J. (1967). Pupillary responses in a pitch-discrimination task. Perception \& Psychophysics, 2, 101-105. Retrieved from http://link.springer. com/article/10.3758/BF03210302

Kahneman, D. \& Treisman, A. M. (1984). Changing views of attention and automaticity. In R. Parasuraman \& D. R. Davies (Eds.), Varieties of attention (pp. 29-61). Orlando: Academic Press.

Kaiser, J. \& Lutzenberger, W. (2005). Human gamma-band activity: A window to cognitive processing. Neuroreport, 16(3), 207-11. doi:10.1097/00001756-20050228000001

Kamienkowski, J. E., Ison, M. J., Quiroga, R. Q., \& Sigman, M. (2012). Fixation-related potentials in visual search: A combined EEG and eye tracking study. Journal of Vision, 12(7), 4. doi:10.1167/12.7.4 
Kastner, S., Pinsk, M. A., De Weerd, P., Desimone, R., \& Ungerleider, L. G. (1999). Increased activity in human visual cortex during directed attention in the absence of visual stimulation. Neuron, 22(4), 751-61. doi:http://dx.doi.org/10.1016/ S0896-6273(00)80734-5

Kimura, F. (1999). Acetylcholine suppresses the spread of excitation in the visual cortex revealed by optical recording: Possible differential effect depending on the source of input. European Journal of Neuroscience, 11(10), 3597-3609. doi:10.1046/j.1460-9568.1999.00779.x

Kirk, R. E. \& Hecht, E. (1963). Maintenance of vigilance by programmed noise. Perceptual and Motor Skills, 16(2), 553-560. doi:10.2466/pms.1963.16.2.553

Klein, R. M. (1988). Inhibitory tagging system facilitates visual search. Nature, 336, 403-405.

Klein, R. M. \& MacInnes, W. J. (1999). Inhibition of return is a foraging facilitator in visual search. Psychological Science, 10, 346-352. doi:10.1111/1467-9280.00166

Klingner, J. (2010a). Fixation-aligned pupillary response averaging. In Proceedings of the 2010 symposium on eye-tracking research \& applications (pp. 275-282). Austin, TX: ACM. doi:10.1145/1743666.1743732

Klingner, J. (2010b). Measuring cognitive load during visual tasks by combining pupillometry and eye tracking (Doctoral dissertation).

Klingner, J., Tversky, B., \& Hanrahan, P. (2011). Effects of visual and verbal presentation on cognitive load in vigilance, memory, and arithmetic tasks. Psychophysiology, 48(3), 323-332. doi:10.1111/j.1469-8986.2010.01069.x

Kloosterman, N. A., Meindertsma, T., van Loon, A. M., Lamme, V. A. F., Bonneh, Y. S., \& Donner, T. H. (2015). Pupil size tracks perceptual content and surprise. European Journal of Neuroscience, 41(8), 1068-1078. doi:10.1111/ejn.12859

Knapen, T., de Gee, J. W., Brascamp, J., Nuiten, S., Hoppenbrouwers, S., \& Theeuwes, J. (2016). Cognitive and ocular factors jointly determine pupil responses under equiluminance. PLoS ONE, 11(5), e0155574. doi:10.1371/journal.pone.0155574

Knudsen, E. I. (2007). Fundamental components of attention. Annual Review of Neuroscience, 30, 57-78. doi:10.1146/annurev.neuro.30.051606.094256

Kobayashi, M., Imamura, K., Sugai, T., Onoda, N., Yamamoto, M., Komai, S., \& Watanabe, Y. (2000). Selective suppression of horizontal propagation in rat visual cortex by norepinephrine. European Journal of Neuroscience, 12(1), 264-272. doi:10.1046/j.1460-9568.2000.00917.x 
Koelega, H. S. (1992). Extraversion and vigilance performance: 30 years of inconsistencies. Psychological Bulletin, 112(2), 239-258. doi:10.1037/0033-2909.112.2.239

Kohn, M. \& Clynes, M. (1969). Color dynamics of the pupil. Annals of the New York Academy of Sciences, 156(2), 931-950. doi:10.1111/j.1749-6632.1969.tb14024.x

Köles, M. (2017). A review of pupillometry for human-computer interaction studies. Periodica Polytechnica Electrical Engineering and Computer Science, 61(4), 320-326. doi:10.3311/PPee.10736

Körner, C. \& Gilchrist, I. D. (2007). Finding a new target in an old display: Evidence for a memory recency effect in visual search. Psychonomic Bulletin \& Review, 14(5), 846-851. doi:10.3758/BF03194110

Körner, C. \& Gilchrist, I. D. (2008). Memory processes in multiple-target visual search. Psychological Research, 72(1), 99-105. doi:10.1007/s00426-006-0075-1

Koss, M. C. (1986). Pupillary dilation as an index of central nervous system alpha 2-adrenoceptor activation. Journal of Pharmacological Methods, 15(1), 1-19. doi:10.1016/0160-5402(86)90002-1

Kostov, A. \& Polak, M. (2000). Parallel man-machine training in development of EEGbased cursor control. IEEE Transactions on Rehabilitation Engineering, 8(2), 203-205. doi:10.1109/86.847816

Kowler, E. (2011). Eye movements: The past 25 years. Vision Research, 51(13), 145783. doi:10.1016/j.visres.2010.12.014

Kowler, E., Anderson, E., Dosher, B. A., \& Blaser, E. (1995). The role of attention in the programming of saccades. Vision Research, 35(13), 1897-1916. doi:10.1016/ 0042-6989(94)00279-U

Krauzlis, R. J., Lovejoy, L. P., \& Zénon, A. (2013). Superior colliculus and visual spatial attention. Annual Review of Neuroscience, 36(May), 165-182. doi:10. 1146/annurev-neuro-062012-170249

Kristjansson, S. D., Stern, J. A., Brown, T. B., \& Rohrbaugh, J. W. (2009). Detecting phasic lapses in alertness using pupillometric measures. Applied Ergonomics, 40(6), 978-986. doi:10.1016/j.apergo.2009.04.007

Kruijne, W. \& Meeter, M. (2016). Implicit short- and long-term memory direct our gaze in visual search. Attention, Perception, \& Psychophysics, 78, 761-773. doi:10.3758/s13414-015-1021-3

Kurzban, R., Duckworth, A., Kable, J. W., \& Myers, J. (2013). An opportunity cost model of subjective effort and task performance. Behavioral and Brain Sciences, 36(06), 661-679. doi:10.1017/S0140525X12003196 
Laeng, B. \& Endestad, T. (2012). Bright illusions reduce the eye's pupil. Proceedings of the National Academy of Sciences, USA, 109(6), 2162-7. doi:10.1073/pnas. 1118298109

Laeng, B. \& Falkenberg, L. (2007). Women's pupillary responses to sexually significant others during the hormonal cycle. Hormones and Behavior, 52(4), 520-30. doi:10. 1016/j.yhbeh.2007.07.013

Laeng, B., Ørbo, M., Holmlund, T., \& Miozzo, M. (2011). Pupillary Stroop effects. Cognitive Processing, 12(1), 13-21. doi:10.1007/s10339-010-0370-z

Laeng, B., Sæther, L., Holmlund, T., Wang, C. E. A., Waterloo, K., Eisemann, M., \& Halvorsen, M. (2013). Invisible emotional expressions influence social judgments and pupillary responses of both depressed and non-depressed individuals. Frontiers in Psychology, 4(May), 291. doi:10.3389/fpsyg.2013.00291

Laeng, B., Sirois, S., \& Gredeback, G. (2012). Pupillometry: a window to the preconscious? Perspectives on Psychological Science, 7(1), 18-27. doi:10.1177/ 1745691611427305

Laeng, B. \& Sulutvedt, U. (2014). The eye pupil adjusts to imaginary light. Psychological Science, 25(1), 188-197. doi:10.1177/0956797613503556

Land, M. F. (2012). Oculomotor behaviour in vertebrates and invertebrates. In The oxford handbook of eye movements. Oxford: Oxford University Press. doi:10. 1093/oxfordhb/9780199539789.013.0001

Land, M. F. \& Furneaux, S. (1997). The knowledge base of the oculomotor system. Philosophical Transactions of the Royal Society of London B: Biological Sciences, 352(1358), 1231-1239. doi:10.1098/rstb.1997.0105

Land, M. F. \& Hayhoe, M. M. (2001). In what ways do eye movements contribute to everyday activities? Vision Research, 41(25-26), 3559-3565. doi:10.1016/S00426989(01)00102-X

Land, M. F. \& McLeod, P. (2000). From eye movements to actions: How batsmen hit the ball. Nature Neuroscience, 3(12), 1340-1345. doi:10.1038/81887

Land, M. F., Mennie, N., \& Rusted, J. (1999). The roles of vision and eye movements in the control of activities of daily living. Perception, 28(11), 1311-1328. doi:10. $1068 / \mathrm{p} 2935$

Land, M. F. \& Tatler, B. W. (2001). Steering with the head: The visual strategy of a racing driver. Current Biology, 11(15), 1215-1220. doi:10.1016/S0960-9822(01)00351- 
Langner, R. \& Eickhoff, S. B. (2013). Sustaining attention to simple tasks: A metaanalytic review of the neural mechanisms of vigilant attention. Psychological Bulletin, 139(4), 870-900. doi:10.1037/a0030694

Lauer, R. T., Peckham, P. H., Kilgore, K. L., \& Heetderks, W. J. (2000). Applications of cortical signals to neuroprosthetic control: A critical review. IEEE Transactions on Rehabilitation Engineering, 8(2), 205-208. doi:10.1109/86.847817

Laughlin, S. B. (1992). Retinal information capacity and the function of the pupil. Ophthalmic \& Physiological Optics, 12(2), 161-164. doi:10.1111/j.14751313.1992.tb00281.x

Lebedev, M. A. \& Nicolelis, M. A. L. (2006). Brain-machine interfaces: Past, present and future. Trends in Neurosciences, 29(9), 536-546. doi:10.1016/j.tins.2006.07. 004

Leknes, S., Wessberg, J., Ellingsen, D.-M., Chelnokova, O., Olausson, H., \& Laeng, B. (2013). Oxytocin enhances pupil dilation and sensitivity to 'hidden' emotional expressions. Social Cognitive and Affective Neuroscience, 8(7), 741-9. doi:10. 1093/scan/nss062

Leys, C., Klein, O., Bernard, P., \& Licata, L. (2013). Detecting outliers: Do not use standard deviation around the mean, use absolute deviation around the median. Journal of Experimental Social Psychology, 49(4), 764-766. doi:10.1016/j.jesp. 2013.03.013

Li, Z. (2002). A saliency map in primary visual cortex. Trends in Cognitive Sciences, 6(1), 9-16.

Libby, W. L. J., Lacey, B. C., \& Lacey, J. I. (1973). Pupillary and cardiac activity during visual attention. Psychophysiology, 10(3), 270-294.

Libet, B., Gleason, C. A., Wright, E. W., \& Pearl, D. K. (1983). Time of conscious intention to act in relation to onset of cerebral activity (readiness-potential): The unconscious initiation of a freely voluntary act. Brain, 106(3), 623-642. doi:10.1093/brain/106.3.623

Lisper, H. O. \& Ericsson, S. (1972). Effects of signal frequency on increase in reaction time in a 10-minute auditory monitoring task. Journal of Experimental Psychology, 98(2), 316-319. doi:10.1037/h0034360

Loeb, M. \& Binford, J. R. (1963). Some factors influencing the effective auditory intensive difference limen. Journal of the Acoustical Society of America, 35(6), 884-891. doi:http://dx.doi.org/10.1121/1.1918621 
Loewenfeld, I. E. (1966). Comment on Hess' findings. Survey of Ophthalmology, 11, 291-294.

Loewenfeld, I. E. (1993). The pupil: Anatomy, physiology and clinical applications. Oxford: Butterworth-Heinemann.

Loftus, G. R. \& Mackworth, N. H. (1978). Cognitive determinants of fixation location during picture viewing. Journal of Experimental Psychology: Human Perception and Performance, 4(4), 565-572. doi:10.1037/0096-1523.4.4.565

Logothetis, N. K. (2008). What we can do and what we cannot do with fMRI. Nature, 453(7197), 869-878. doi:nature06976[pii]ไr10.1038/nature06976

Logothetis, N. K., Pauls, J., Augath, M., Trinath, T., \& Oeltermann, A. (2001). Neurophysiological investigation of the basis of the fMRI signal. Nature, 412(6843), 150-7. doi:10.1038/35084005

Loh, S., Lamond, N., Dorrian, J., Roach, G. D., \& Dawson, D. (2004). The validity of psychomotor vigilance tasks of less than 10-minute duration. Behavior Research Methods, Instruments, \& Computers, 36(2), 339-346. doi:10.3758/BF03195580

Lorber, M., Zuber, B. L., \& Stark, L. (1965). Suppression of the pupillary light reflex in binocular rivalry and saccadic suppression. Nature, 208, 558-560. doi:10.1038/ $208558 \mathrm{a} 0$

Lowenstein, O., Feinberg, R., \& Loewenfeld, I. E. (1963). Pupillary movements during acute and chronic fatigue a new test for the objective evaluation of tiredness. Investigative Ophthalmology \& Visual Science, (1). Retrieved from http://www. iovs.org/content/2/2/138.short

Lowenstein, O. \& Loewenfeld, I. E. (1964). The sleep-waking cycle and pupillary activity. Annals of the New York Academy of Sciences, 117, 142-156.

Lubow, R. E. (1996). Pupillary size in response to a visual guilty knowledge test: New technique for the detection of deception. Journal of Experimental Psychology: Applied, 2(2), 164-177. doi:10.1037//1076-898X.2.2.164

Lucaccini, L. F., Freedy, A., \& Lyman, J. (1968). Motivational factors in vigilance: Effects of instructions on performance in a complex vigilance task. Perceptual and Motor Skills, 26, 783-786. doi:10.2466/pms.1968.26.3.783

Luck, S. J. (2008). Visual short-term memory. In S. J. Luck \& A. Hollingworth (Eds.), Visual memory (pp. 43-85). New York: Oxford University Press. doi:10.1093/ acprof:oso/9780195305487.003.0003 
Luck, S. J. (2014). An introduction to the event related potential technique. In $A n$ introduction to the event related potential technique (2nd). Boston: MIT press. doi:10.1111/mono.12122

Luck, S. J. \& Vogel, E. K. (1997). The capacity of visual working memory for features and conjunctions. Nature, 390(6657), 279-281. doi:10.1038/36846

Luck, S. J. \& Vogel, E. K. (2013). Visual working memory capacity: From psychophysics and neurobiology to individual differences. Trends in Cognitive Sciences, 17(8), 391-400. doi:10.1016/j.tics.2013.06.006

Lüdtke, H., Wilhelm, B., Adler, M., Schaeffel, F., \& Wilhelm, H. (1998). Mathematical procedures in data recording and processing of pupillary fatigue waves. Vision Research, 38(19), 2889-2896. doi:10.1016/S0042-6989(98)00081-9

Lynn, R. (1966). Attention, arousal and the orientation reaction. Oxford, UK: Pergamon Press.

Mackie, R. R. (1987). Vigilance research-are we ready for countermeasures? Human Factors, 29(6), 707-723. doi:10.1177/001872088702900610

Mackworth, J. F. (1970). Vigilance and attention. Baltimore: Penguin.

Mackworth, N. H. (1948). The breakdown of vigilance durning prolonged visual search. The Quarterly Journal of Experimental Psychology, 1(1), 6-21. doi:10.1080/ 17470214808416738

Mackworth, N. H. (1950). Researches on the measurement of human performance. London: H. M. S. O.

Mackworth, N. H. \& Morandi, A. J. (1967). The gaze selects information details within picutres. Perception \& Psychophysics, 2(11), 547-552.

MacLean, K. A., Aichele, S. R., Bridwell, D. A., Mangun, G. R., Wojciulik, E., \& Saron, C. D. (2009). Interactions between endogenous and exogenous attention during vigilance. Attention, Perception, \& Psychophysics, 71(5), 1042-1058. doi:10.3758/APP

MacLeod, C. M. (1991). Half a century of research on the Stroop effect: An integrative review. Psychological Bulletin, 109(2), 163-203. doi:10.1037/0033-2909.109.2. 163

MacMillan, N. A. (2001). Signal detection theory. In Stevens' handbook of experimental psychology (Vol. 4, pp. 43-90). doi:10.1002/0471214426

MacMillan, N. A. \& Creelman, C. D. (2005). Detection theory: A user's guide (2nd ed.). doi:10.1017/CBO9781107415324.004 
Magliero, A. (1983). Pupil dilations following pairs of identical and related to-beremembered words. Memory \& Cognition, 11(6), 609-615.

Majaranta, P. \& Räihä, K.-J. (2002). Twenty years of eye typing: Systems and design issues. In Proceedings of eye tracking research and applications (Vol. 1, 212, pp. 15-22). New Orleans, LA: ACM. doi:10.1145/507072.507076

Makeig, S. \& Inlow, M. (1993). Lapse in alertness: Coherence of fluctuations in performance and EEG spectrum. Electroencephalography and Clinical Neurophysiology, 86(1), 23-35. doi:10.1016/0013-4694(93)90064-3

Manly, T., Robertson, I. H., Galloway, M., \& Hawkins, K. (1999). The absent mind: Further investigations of sustained attention to response. Neuropsychologia, 37(6), 661-670. doi:10.1016/S0028-3932(98)00127-4

Manousakis, J., Maccora, J., Ftouni, S., \& Anderson, C. (2017). It's in the eyes: Pupillary response provides a physiological marker of alertness and performance impairment. Journal of Sleep Research, 26(Suppl. 1), 28. doi:10.1111/jsr.59_12618

Maris, E. \& Oostenveld, R. (2007). Nonparametric statistical testing of EEG- and MEG-data. Journal of Neuroscience Methods, 164(1), 177-190. doi:10.1016/j. jneumeth.2007.03.024

Marr, D. (1982). Vision. Retrieved from http :// books . google $\cdot$ com / books ? id= EehUQwAACAAJ\%7B\%5C\&\%7Dprintsec=frontcover\%7B\%5C\%\%7D5Cnpapers2: //publication/uuid/FBD15E5F-E503-450B-B059-4C15D54099CE

Marshall, S. P. (2002). The Index of Cognitive Activity: Measuring cognitive workload. Proceedings of the IEEE 7th Conference on Human Factors and Power Plants, 7-5-7-9. doi:10.1109/HFPP.2002.1042860

Marshall, S. P., Pleydell-Pearce, C. W., \& Dickson, B. T. (2003). Integrating psychophysiological measures of cognitive workload and eye movements to detect strategy shifts. Proceedings of the 36th Annual Hawaii International Conference on System Sciences, HICSS 2003, 3-8. doi:10.1109/HICSS.2003.1174298

Martinez-Conde, S., Macknik, S. L., \& Hubel, D. H. (2000). Microsaccadic eye movements and firing of single cells in the striate cortex of macaque monkeys. Nature Neuroscience, 3(3), 251-258. doi:10.1038/72961

Martinez-Conde, S., Macknik, S. L., \& Hubel, D. H. (2004). The role of fixational eye movements in visual perception. Nature Reviews Neuroscience, 5(3), 229-240. doi: $10.1038 / \mathrm{nrn} 1348$

Mason, S. T. \& Iversen, S. D. (1978). Reward, attention and the dorsal noradrenergic bundle. Brain Research, 150(1), 135-148. doi:10.1016/0006-8993(78)90658-3 
Massar, S. A. A., Lim, J., Sasmita, K., \& Chee, M. W. L. (2016). Rewards boost sustained attention through higher effort: A value-based decision making approach. Biological Psychology, 120, 21-27. doi:10.1016/j.biopsycho.2016.07.019

Mather, M. \& Harley, C. W. (2016). The locus coeruleus: Essential for maintaining cognitive function and the aging brain. Trends in Cognitive Sciences, 20(3), 214226. doi:10.1016/j.tics.2016.01.001

Mathôt, S. (2013). A simple way to reconstruct pupil size during eye blinks. Figshare, 1-4. doi:10.6084/m9.figshare.688001

Mathôt, S., Fabius, J. H., Van Heusden, E., \& Van der Stigchel, S. (2018). Safe and sensible baseline correction of pupil-size data. Behavior Research Methods, (April), 1-25. doi:10.3758/s13428-017-1007-2

Mathôt, S., Grainger, J., \& Strijkers, K. (2017). Pupillary responses to words that convey a sense of brightness or darkness. Psychological Science, 28(8), 11161124. doi:10.1177/0956797617702699

Mathôt, S., Melmi, J.-B., \& Castet, E. (2015). Intrasaccadic perception triggers pupillary constriction. PeerJ, 3, e1150. doi:10.7717/peerj.1150

Mathôt, S., Melmi, J.-B., van der Linden, L., \& Van der Stigchel, S. (2015). The mind-writing pupil: Near-perfect decoding of visual attention with pupillometry. Journal of Vision, 15(12), 176. doi:10.1167/15.12.176

Mathôt, S., Melmi, J.-B., van der Linden, L., \& Van der Stigchel, S. (2016). The mindwriting pupil: A human-computer interface based on decoding of covert attention through pupillometry. PLOS ONE, 11(2), e0148805. doi:10.1371/journal.pone. 0148805

Mathôt, S., Siebold, A., Donk, M., Vitu, F., \& Siebold, A. (2015). Large pupils predict goal-driven eye movements. Journal of Experimental Psychology: General. doi:http://dx.doi.org/10.1037/a0039168

Mathôt, S., Umr, L. P. C., Dalmaijer, E. S., Grainger, J., \& Stigchel, S. V. D. (2014). The pupillary light response reflects exogenous attention and inhibition of return. Journal of Vision, 14(7), 1-9. doi:10.1167/14.14.7.doi

Mathôt, S., van der Linden, L., Grainger, J., \& Vitu, F. (2013). The pupillary light response reveals the focus of covert visual attention. PLoS ONE, 8(10), e78168. doi:10.1371/journal.pone.0078168

Mathôt, S., van der Linden, L., Jonathan, G., \& Vitu, F. (2014). The pupillary light response reflects eye-movement preparation. Journal of Vision, 14(10), 203-203. doi:10.1167/14.10.203 
Matsangas, P., Shattuck, N. L., \& Brown, S. (2016). Preliminary validation study of the 3-min wrist-worn psychomotor vigilance test. Behavior Research Methods, 1-10. doi:10.3758/s13428-016-0821-2

Maunsell, J. H. R. \& Newsome, W. T. (1987). Visual processing in monkey extrastriate cortex.

McBride, S. A., Merullo, D. J., Johnson, R. F., Banderet, L. E., \& Robinson, R. T. (2007). Performance during a 3-hour simulated sentry duty task under varied work rates and secondary task demands. Military Psychology, 19(2), 103-117. doi:10.1080/08995600701323392

McClelland, L. E., Pilcher, J. J., \& Moore, D. D. (2010). Oculomotor measures as predictors of performance during sleep deprivation. Aviation Space and Environmental Medicine, 81(9), 833-842. doi:10.3357/ASEM.2653.2010

McGregor, D. K. \& Stern, J. A. (1996). Time on task and blink effects on saccade duration. Ergonomics, 39(4), 649-60. doi:10.1080/00140139608964487

McIntire, L. K., McKinley, R. A., \& Goodyear, C. (2014). Detection of vigilance performance with pupillometry. In Applied proceedings of the symposium on eye tracking research and applications (pp. 167-174). ACM. doi:10.1145/2578153. 2578177

McIntire, L. K., McKinley, R. A., Goodyear, C., \& McIntire, J. P. (2014). Detection of vigilance performance using eye blinks. Applied Ergonomics, 45(2), 354-62. doi:10.1016/j.apergo.2013.04.020

McLaren, J. W., Erie, J. C., \& Brubaker, R. F. (1992). Computerized analysis of pupillograms in studies of alertness. Investigative Ophthalmology \& Visual Science, 33(3), 671-6. Retrieved from http://www.ncbi.nlm.nih.gov/pubmed/1544791

McLeod, P., Driver, J., \& Crisp, J. (1988). Visual search for a conjunction of movement and form is parallel. Nature, 332(6160), 154-155. doi:10.1038/332154a0

McPeek, R. M. \& Keller, E. L. (2004). Deficits in saccade target selection after inactivation of superior colliculus. Nature Neuroscience, 7(7), 757-63. doi:10.1038/ nn1269

McVay, J. C. \& Kane, M. J. (2009). Conducting the train of thought: Working memory capacity, goal neglect, and mind wandering in an executive-control task. Journal of Experimental Psychology: Learning, Memory, and Cognition, 35(1), 196-204. doi:10.1037/a0014104 
McVay, J. C. \& Kane, M. J. (2010). Does mind wandering reflect executive function or executive failure? Comment on Smallwood and Schooler (2006) and Watkins (2008). Psychological Bulletin, 136(2), 188-197. doi:10.1037/a0018298

McVay, J. C. \& Kane, M. J. (2012). Drifting from slow to “d'oh!”: Working memory capacity and mind wandering predict extreme reaction times and executive control errors. Journal of Experimental Psychology: Learning, Memory, and Cognition, 38(3), 525-549. doi:10.1037/a0025896

Menneer, T., Barret, D. K., Phillips, L., Donnelly, N., \& Cave, K. R. (2007). Costs in searching for two targets: Dividing search across target types could improve airport security screening. Applied Cognitive Psychology, 21(December 2006), 915-932. doi:10.1002/acp.1305

Menneer, T., Cave, K. R., \& Donnelly, N. (2009). The cost of search for multiple targets: Effects of practice and target similarity. Journal of Experimental Psychology: Applied, 15(2), 125-139. doi:10.1037/a0015331

Menneer, T., Donnelly, N., Godwin, H. J., \& Cave, K. R. (2010). High or low target prevalence increases the dual-target cost in visual search. Journal of Experimental Psychology: Applied, 16(2), 133-44. doi:10.1037/a0019569

Metzger, K. R., Warm, J. S., \& Senter, R. J. (1974). Effects of background event rate and critical signal amplitude on vigilance performance. Perceptual and Motor Skills, 38(3, Pt 2), 1175-1181. doi:https://doi.org/10.2466/pms.1974.38.3c.1175

Meyrand, P., Simmers, J., \& Moulins, M. (1994). Dynamic construction of a neural network from multiple pattern generators in the lobster stomatogastric nervous system. The Journal of neuroscience : the official journal of the Society for Neuroscience, 14(2), 630-44. Retrieved from http://www.ncbi.nlm.nih.gov/ pubmed/7507982

Miller, G. A. (1956). The magical number 7, plus or minus 2 - some limits on our capacity for processing information. Psychological Review, 63(2), 81-97. doi:10. 1037/h0043158

Miller, G. A. (2003). The cognitive revolution: A historical perspective. Trends in Cognitive Sciences, 7(3), 141-144. doi:10.1016/S1364-6613(03)00029-9

Mittner, M., Boekel, W., Tucker, A. M., Turner, B. M., Heathcote, A., \& Forstmann, B. U. (2014). When the brain takes a break: A model-based analysis of mind wandering. The Journal of Neuroscience, 34(49), 16286-16295. doi:10.1523/ JNEUROSCI.2062-14.2014 
Mittner, M., Hawkins, G. E., Boekel, W., \& Forstmann, B. U. (2016). A neural model of mind wandering. Trends in Cognitive Sciences, 20(8), 570-578. doi:10.1016/j. tics.2016.06.004

Miyake, A. \& Shah, P. (1999). Models of working memory: Mechanisms of active maintenance and executive control. New York: Cambridge University Press. doi:10.1017/CBO9781139174909

Miyawaki, Y., Uchida, H., Yamashita, O., Sato, M.-a., Morito, Y., \& Tanabe, H. C. (2008). Visual image reconstruction from human brain activity using a combination of multiscale local image decoders. Neuron, 60(5), 915-929. doi:10.1016/j. neuron.2008.11.004

Monsell, S. (1996). Control of mental processes. In Unsolved mysteries of the mind: Tutorial essays in cognition (pp. 93-148). Hove, UK: Erlbaum.

Montefinese, M., Ambrosini, E., Fairfield, B., \& Mammarella, N. (2013). The "subjective" pupil old/new effect: Is the truth plain to see? International Journal of Psychophysiology, 89(1), 48-56. doi:10.1016/j.ijpsycho.2013.05.001

Morad, Y., Lemberg, H., Yofe, N., \& Dagan, Y. (2000). Pupillography as an objective indicator of fatigue. Current Eye Research, 21(1), 535-542. doi:citeulike-articleid:10485869\rdoi:10.1076/0271-3683(200007)2111-ZFT535

Moray, N. (1969). Attention: selective processes in vision and hearing. London: Hutchinson Educational.

Morris, T. L. (1996). Electrooculographic and performance indices of fatigue during simulated flight. Biological Psychiatry, 42, 343-360.

Müller, H. J. \& von Mühlenen, A. (2000). Probing distractor inhibition in visual search: Inhibition of return. Journal of Experimental Psychology: Human Perception and Performance, 26(5), 1591-1605. doi:10.1037/0096-1523.26.5.1591

Murphy, P. R., O’Connell, R. G., O’Sullivan, M., Robertson, I. H., \& Balsters, J. H. (2014). Pupil diameter covaries with BOLD activity in human locus coeruleus. Human Brain Mapping, 4154, 4140-4154. doi:10.1002/hbm.22466

Murphy, P. R., Robertson, I. H., Balsters, J. H., \& O’Connell, R. G. (2011). Pupillometry and P3 index the locus coeruleus-noradrenergic arousal function in humans. Psychophysiology, 48(11), 1532-43. doi:10.1111/j.1469-8986.2011.01226.x

Mysore, S. P. \& Knudsen, E. I. (2013). A shared inhibitory circuit for both exogenous and endogenous control of stimulus selection. Nature Neuroscience, 16(4), 473-8. doi:10.1038/nn.3352 
Naber, M., Alvarez, G. A., \& Nakayama, K. (2013). Tracking the allocation of attention using human pupillary oscillations. Frontiers in Psychology, 4(December), 919. doi:10.3389/fpsyg.2013.00919

Naber, M., Einhäuser, W., \& Frassle, S. (2011). Perceptual rivalry: Reflexes reveal the gradual nature of visual awareness. PLoS ONE, 6(6), e20910. doi:10.1371/journal. pone. 0020910

Naber, M., Frässle, S., Rutishauser, U., \& Einhäuser, W. (2013). Pupil size signals novelty and predicts later retrieval success for declarative memories of natural scenes. Journal of Vision, 13(2), 11. doi:10.1167/13.2.11.doi

Naber, M. \& Nakayama, K. (2013). Pupil responses to high-level image content. Journal of Vision, 13, 1-8. doi:10.1167/13.6.7.doi

Nagai, M., Wada, M., \& Sunaga, N. (2002). Trait anxiety affects the pupillary light reflex in college students. Neuroscience Letters, 328, 68-70.

Nagy, A. L. \& Sanchez, R. R. (1990). Critical color differences determined with a visual search task. Journal of the Optical Society of America: A, 7(7), 1209-17. doi:10.1364/JOSAA.7.001209

Najemnik, J. \& Geisler, W. S. (2005). Optimal eye movement strategies in visual search. Nature, 434(7031), 387-391. doi:10.1167/5.8.778

Nakagawa, S. (2004). A farewell to Bonferroni: the problems of low statistical power and publication bias. Behavioral Ecology, 15(6), 1044-1045. doi:10.1093/beheco/ $\operatorname{arh} 107$

Nakayama, K. \& Joseph, J. S. (1998). Attention, pattern recognition and pop-out in visual search. In The attentive brain (pp. 279-298).

Nakayama, K. \& Silverman, G. H. (1986). Serial and parallel processing of visual feature conjunctions. doi:10.1038/320264a0

Nakayama, M., Takahashi, K., \& Shimizu, Y. (2002). The act of task difficulty and eyemovement frequency for the 'oculo-motor indices'. In Proceedings of the 2002 symposium on eye-tracking research \& applications (pp. 37-42). New Orleans: ACM.

Neisser, U. (1967). Cognitive psychology. New York: Meredith Publishing Company.

Netser, S., Dutta, A., \& Gutfreund, Y. (2014). Ongoing activity in the optic tectum is correlated on a trial-by-trial basis with the pupil dilation response. Journal of Neurophysiology, 111(5), 918-29. doi:10.1152/jn.00527.2013 
Netser, S., Ohayon, S., \& Yoram, G. (2010). Multiple manifestations of microstimulation in the optic tectum: Eye movements, pupil dilations, and sensory priming. Journal of Neurophysiology, 104, 108-112. doi:10.1152/jn.01142.2009

Neuman, R. S. \& Harley, C. W. (1983). Long-lasting potentiation of the dentate gyrus population spike by norepinephrine. Brain Research, 273(1), 162-165. doi:10. 1016/0006-8993(83)91106-X

Neuper, C., Müller, G. R., Kübler, A., Birbaumer, N., \& Pfurtscheller, G. (2003). Clinical application of an EEG-based brain-computer interface: A case study in a patient with severe motor impairment. Clinical Neurophysiology, 114(3), 399-409. doi:10.1016/S1388-2457(02)00387-5

Newell, A. \& Card, S. K. (1985). The prospect for psychological science in humancomputer interaction. doi:10.1207/s15327051hci0103_1

Nieuwenhuis, S., de Geus, E. J., \& Aston-Jones, G. (2010). The anatomical and functional relationship between the P3 and autonomic components of the orienting response. Psychophysiology, 48(2), 162-175. doi:10.1111/j.1469-8986.2010. 01057.x

Nishimoto, S., Vu, A. T., Naselaris, T., Benjamini, Y., Yu, B., \& Gallant, J. L. (2011). Report reconstructing visual experiences from brain activity evoked by natural movies. Current Biology, 21, 1641-1646. doi:10.1016/j.cub.2011.08.031

Nothdurft, H. (2000). Salience from feature contrast: Additivity across dimensions. Vision Research, 40(10-12), 1183-1201. doi:10.1016/S0042-6989(00)00031-6

Nuske, H. J., Vivanti, G., Hudry, K., \& Dissanayake, C. (2014). Pupillometry reveals reduced unconscious emotional reactivity in autism. Biological Psychology, 101, 24-35. doi:10.1016/j.biopsycho.2014.07.003

Nuthmann, A. \& van der Meer, E. (2005). Time's arrow and pupillary response. Psychophysiology, 42(3), 306-17. doi:10.1111/j.1469-8986.2005.00291.x

Ogawa, S., Lee, T. M., Kay, A. R., \& Tank, D. W. (1990). Brain magnetic resonance imaging with contrast dependent on blood oxygenation. Proceedings of the $\mathrm{Na}$ tional Academy of Sciences, USA, 87(24), 9868-72. doi:10.1073/pnas.87.24.9868

Ogawa, S., Tank, D. W., Menon, R., Ellermann, J. M., Kim, S. G., Merkle, H., \& Ugurbil, K. (1992). Intrinsic signal changes accompanying sensory stimulation: Functional brain mapping with magnetic resonance imaging. Proceedings of the National Academy of Sciences, USA, 89(13), 5951-5955. doi:10.1073/pnas.89.13.5951

O’Hanlon, J. F. \& Beatty, J. (1977). Concurrence of electroencephalographic and performance changes during a simulated radar watch and some implications 
for the arousal theory of vigilance. In R. R. Mackie (Ed.), Vigilance: Theory, operational performance and physiological correlates. New York: Plenum.

Oken, B. S., Salinsky, M. C., \& Elsas, S. M. (2006). Vigilance, alertness, or sustained attention: physiological basis and measurement. Clinical Neurophysiology, 117(9), 1885-901. doi:10.1016/j.clinph.2006.01.017

O’Reilly, R. C., Braver, T. S., \& Cohen, J. D. (1999). A biologically-based computational model of working memory. In A. Miyake \& P. Shah (Eds.), Models of working memory: Mechanisms of active maintenance and executive control (pp. 375-411). New York: Cambridge University Press. doi:10.1017/CBO9781139174909

Otero, S. C., Weekes, B. S., \& Hutton, S. B. (2011). Pupil size changes during recognition memory. Psychophysiology, 48(10), 1346-1353. doi:10.1111/j.14698986.2011.01217.x

Paivio, A. \& Simpson, H. M. (1966). The effects of word abstractness and pleasantness on pupil size during an imagery task. Psychonomic Science, 5(2), 55-56. Retrieved from http://ezproxy.net.ucf.edu/login?url=http://search.ebscohost.com/login. aspx?direct=true \%7B\%5C\&\%7Ddb=psyh\%7B\%5C\&\%7DAN=2006-99014064\%7B\%5C\&\%7Dsite=ehost-live

Palmer, J. (1994). Set-size effects in visual search: The effect of attention is independent of the stimulus for simple tasks. Vision Research, 34(13), 1703-1721. doi:10. 1016/0042-6989(94)90128-7

Palmer, J., Ames, C. T., \& Lindsey, D. T. (1993). Measuring the effect of attention on simple visual search. doi:10.1037/0096-1523.19.1.108

Palmer, J., Verghese, P., \& Pavel, M. (2000). The psychophysics of visual search. Vision Research, 40, 1227-1268. doi:10.1016/S0042-6989(99)00244-8

Palmer, T. E. (1975). The effects of contextual scenes on the identification of objects. Memory \& Cognition, 3(5), 519-526. doi:10.3758/BF03197524

Papesh, M. H., Goldinger, S. D., \& Hout, M. C. (2012). Memory strength and specificity revealed by pupillometry. International Journal of Psychophysiology, 83(1), 5664. doi:10.1016/j.ijpsycho.2011.10.002

Parasuraman, R. (1979). Memory load and event rate control sensitivity decrements in sustained attention. Science, 205(4409), 924-7. doi:10.1126/science.472714

Parasuraman, R. (1986). Vigilance, monitoring, and search. New York: Wiley. Retrieved from http://ovidsp.ovid.com/ovidweb.cgi $? \mathrm{~T}=\mathrm{JS} \%$ 7B \% 5C \& \% 7DPAGE = reference $\% 7 \mathrm{~B} \% 5 \mathrm{C} \& \% 7 \mathrm{DD}=$ psyc $2 \% 7 \mathrm{~B} \% 5 \mathrm{C} \& \% 7 \mathrm{DNEWS}=\mathrm{N} \% 7 \mathrm{~B} \% 5 \mathrm{C} \&$ \%7DAN=1986-98619-021 
Parasuraman, R. (1987). Human-computer monitoring. Human Factors, 29(6), 695-706. doi:10.1177/001872088702900609

Parasuraman, R. (1998). The attentive brain: Issues and prospects. In R. Parasuraman (Ed.), The attentive brain (Chap. 1). Boston: MIT press.

Parasuraman, R. \& Davies, D. R. (1976). Decision theory analysis of response latencies in vigilance. Journal of Experimental Psychology: Human Perception and Performance, 2(4), 578-90. Retrieved from http://www.ncbi.nlm.nih.gov/pubmed/ 1011007

Parasuraman, R. \& Davies, D. R. (1977). A taxonomic analysis of vigilance performance. In R. R. Mackie (Ed.), Vigilance: Theory, operational performance and physiological correlates (pp. 559-574). New York: Plenum Press. doi:10.1007/978-14684-2529-1_26

Parasuraman, R. \& Davies, D. R. (1982). The psychology of vigilance. London: Academic Press.

Parasuraman, R. \& Davies, D. R. (1984). Varieties of attention. San Diego, CA: Academic Press.

Parasuraman, R., de Visser, E., Clarke, E., McGarry, W. R., Hussey, E., Shaw, T., \& Thompson, J. C. (2009). Detecting threat-related intentional actions of others: Effects of image quality, response mode, and target cuing on vigilance. Journal of Experimental Psychology: Applied, 15(4), 275-90. doi:10.1037/a0017132

Parasuraman, R. \& Riley, V. (1997). Humans and automation: Use, misuse, disuse, abuse. Human Factors, 39(2), 230-253. doi:10.1518/001872097778543886

Parasuraman, R., Warm, J. S., \& See, J. E. (1998). Brain systems of vigilance. In R. Parasuraman (Ed.), The attentive brain (pp. 221-256). Retrieved from http: $/ /$ search.ebscohost.com/login.aspx?direct=true\%7B\%5C\&\%7Ddb=psyh\%7B\% 5C\&\%7DAN=1998-07668-010\%7B\%5C\&\%7Dsite=ehost-live

Parkhurst, D. J. \& Niebur, E. (2002). Variable-resolution displays: A theoretical, practical, and behavioral evaluation. Human Factors, 44(4), 611-629. doi:10.1518/ 0018720024497015

Partala, T. \& Surakka, V. (2003). Pupil size variation as an indication of affective processing. International Journal of Human-Computer Studies, 59(1-2), 185-198. doi:10.1016/S1071-5819(03)00017-X

Pashler, H. (1988). Familiarity and visual change detection. Perception \& Psychophysics, 44(4), 369-378. doi:10.3758/BF03210419

Pashler, H. (1998). The psychology of attention. Cambridge, MA: MIT press. 
Patla, A. E. \& Vickers, J. N. (2003). How far ahead do we look when required to step on specific locations in the travel path during locomotion? Experimental Brain Research, 148(1), 133-138. doi:10.1007/s00221-002-1246-y

Pavlov, I. P. (1927). Conditioned reflexes. Oxford: Clarendon Press.

Petersen, M. S., Kramer, A. F., Wang, R. F., Irwin, D. E., \& McCarley, J. S. (2001). Visual search has memory. Psychological Science, 12(4), 360-366. doi:10.1111/ 1467-9280.00353

Petersen, S. E. \& Posner, M. I. (2012). The attention system of the human brain: 20 years after. Annual Review of Neuroscience, 35(1), 73-89. doi:10.1146/annurevneuro-062111-150525

Peterson, L. \& Peterson, M. J. (1959). Short-term retention of individual verbal items. Journal of Experimental Psychology, 58(3), 193-198. doi:10.1037/h0049234

Phillips, M. A., Szabadi, E. U., \& Bradshaw, C. M. (2000). Comparison of the effects of clonidine and yohimbine on spontaneous pupillary fluctuations in healthy human volunteers. Psychopharmacology, 150(1), 85-89. doi:10.1007/s002130000398

Phillips, W. A. (1974). On the distinction between sensory storage and short-term visual memory. Perception \& Psychophysics, 16(2), 283-290. doi:10.3758/BF03203943

Pierre, M. (1973). Pupil size and affect: a critical review of the literature since 1960. The Canadian Psychologist, 14(4), 311-329. doi:10.1037/h0082230

Pigeau, R. A., Angus, R. G., O’Neill, P., \& Mack, I. (1995). Vigilance latencies to aircraft detection among NORAD surveillance operators. Human Factors, 37(3), 622-634. doi:10.1518/001872095779049291

Piquado, T., Isaacowitz, D., \& Wingfield, A. (2010). Pupillometry as a measure of cognitive effort in younger and older adults. Psychophysiology, 47(3), 560-569. doi:10.1111/j.1469-8986.2009.00947.x.

Poletti, M., Listorti, C., \& Rucci, M. (2013). Microscopic eye movements compensate for nonhomogeneous vision within the fovea. Current Biology, 23(17), 16911695. doi:10.1016/j.cub.2013.07.007

Poli, R., Valeriani, D., \& Cinel, C. (2014). Collaborative brain-computer interface for aiding decision-making. PLoS ONE, 9(7), e102693. doi:10.1371/journal.pone. 0102693

Pomplun, M. \& Sunkara, S. (2003). Pupil dilation as an indicator of cognitive workload in human-computer interaction. Proceedings of the International Conference on HCI. Retrieved from http://www.researchgate.net/publication/228870489\%7B\% 5C_\%7DPupil\%7B\%5C_\%7Ddilation\%7B\%5C_\%7Das\%7B\%5C_\%7Dan\% 
7B\%5C_\%7Dindicator\%7B\%5C_\%7Dof\%7B\%5C_\%7Dcognitive\%7B\%5C_ \%7Dworkload\%7B\%5C_\%7Din\%7B\%5C_\%7Dhuman-computer\%7B\%5C_ \%7Dinteraction/file/3deec516f11abbb001.pdf

Pomplun, M., Sunkara, S., Fairley, A. V., \& Xiao, M. (2011). Using pupil size as a measure of cognitive workload in video-based eye-tracking studies. Cs.Umb.Edu. Retrieved from http://www.cs.umb.edu/\%7B \% 7Dmarc/pubs/pomplun \% 7B\%5C_\%7Dsunkara\%7B\%5C_\%7Dfairley\%7B\%5C_\%7Dxiao\%7B\%5C_ $\% 7 D d r a f t . p d f$

Poock, G. K. (1973). Information processing in pupil diameter. Perceptual and Motor Skills, 37, 1000-1002.

Porter, G., Leonards, U., Wilcock, G., Haworth, J., Troscianko, T., \& Tales, A. (2010). New insights into feature and conjunction search: II. Evidence from Alzheimer's disease. Cortex, 46(5), 637-649. doi:10.1016/j.cortex.2009.04.014

Porter, G., Tales, A., Troscianko, T., Wilcock, G., Haworth, J., \& Leonards, U. (2010). New insights into feature and conjunction search: I. Evidence from pupil size, eye movements and ageing. Cortex, 46(5), 621-36. doi:10.1016/j.cortex.2009.04.013

Porter, G., Troscianko, T., \& Gilchrist, I. D. (2007). Effort during visual search and counting: Insights from pupillometry. The Quarterly Journal of Experimental Psychology, 60(2), 211-29. doi:10.1080/17470210600673818

Posner, M. I. (1980). Orienting of attention. The Quarterly Journal of Experimental Psychology, 32(1), 3-25. doi:10.1080/00335558008248231

Posner, M. I. \& Boies, S. J. (1971). Components of attention. Psychological Review, 78(5), 391-408. doi:10.1037/h0031333

Posner, M. I. \& Cohen, Y. (1984). Components of visual orienting. Attention and performance X, 531-556. doi:10.1162/jocn.1991.3.4.335

Posner, M. I. \& Petersen, S. E. (1990). The attention system of the human brain. Annual Review of Neuroscience, 13, 25-42. doi:10.1146/annurev.ne.13.030190.000325

Posner, M. I., Snyder, C. R., \& Davidson, B. J. (1980). Attention and the detection of signals. Journal of Experimental Psychology, 109(2), 160-174. doi:10.1037/00963445.109.2.160

Poulton, E. C. (1977). Arousing stresses increase vigilance. In R. R. Mackie (Ed.), Vigilance: Theory, operational performance and physiological correlates. New York: Plenum. 
Poulton, E. C. \& Edwards, R. S. (1974). The interaction of the loss of a night's sleep with mild heat: Task variables. Ergonomics, 17(1), 59-73. doi:10.1080/ 00140137408931313

Prettyman, R., Bitsios, P., \& Szabadi, E. U. (1997). Altered pupillary size and darkness and light reflexes in Alzheimer's disease. Journal of Neurology Neurosurgery and Psychiatry, 62(6), 665-668. doi:10.1136/jnnp.62.6.665

Preuschoff, K., 't Hart, B. M., \& Einhäuser, W. (2011). Pupil dilation signals surprise: evidence for noradrenaline's role in decision making. Frontiers in Neuroscience, 5(SEP), 1-12. doi:10.3389/fnins.2011.00115

Privitera, C. M., Carney, T., Klein, S., \& Aguilar, M. (2014). Analysis of microsaccades and pupil dilation reveals a common decisional origin during visual search. Vision Research, 95, 43-50. doi:10.1016/j.visres.2013.12.001

Privitera, C. M., Renninger, L. W., Carney, T., Klein, S., \& Aguilar, M. (2010). Pupil dilation during visual target detection. Journal of Vision, 10, 1-14. doi:10.1167/ 10.10.3.Introduction

Pyke, G. H., Pulliam, H. R., \& Charnov, E. L. (1977). Optimal foraging: A selective review of theory and tests. The Quarterly Review of Biology, 52(2), 137-154.

Raichle, M. E. (2010). Two views of brain function. Trends in Cognitive Sciences, 14(4), 180-190. doi:10.1016/j.tics.2010.01.008

Raisig, S., Welke, T., Hagendorf, H., \& van der Meer, E. (2010). I spy with my little eye: Detection of temporal violations in event sequences and the pupillary response. International Journal of Psychophysiology, 76(1), 1-8. doi:10.1016/j.ijpsycho. 2010.01.006

Rajkowski, J., Kubiak, P., \& Aston-Jones, G. (1993). Correlations between locus coeruleus (LC) neural activity, pupil diameter and behavior in monkey support a role of LC in attention. Society for Neuroscience Abstracts, 19, 47.

Rajkowski, J., Kubiak, P., \& Aston-Jones, G. (1994). Locus coeruleus activity in monkey: Phasic and tonic changes are associated with altered vigilance. Brain Research Bulletin, 35(5-6), 607-616. doi:10.1016/0361-9230(94)90175-9

Rajkowski, J., Majczynski, H., Clayton, E. C., \& Aston-Jones, G. (2004). Activation of monkey locus coeruleus neurons varies with difficulty and performance in a target detection task. Journal of Neurophysiology, 92(1), 361-371. doi:10.1152/ jn.00673.2003

Ralph, B. C. W., Onderwater, K., Thomson, D. R., \& Smilek, D. (2016). Disrupting monotony while increasing demand: Benefits of rest and intervening tasks on 
vigilance. Psychological Research, 81(1948), 432-444. doi:10.1007/s00426-0160752-7

Raymond, J. E., Shapiro, K. L., \& Arnell, K. M. (1992). Temporary suppression of visual processing in an RSVP task: An attentional blink? Journal of Experimental Psychology: Human Perception and Performance, 18(3), 849-860. doi:10.1037/ 0096-1523.18.3.849

Rayner, K. (1978). Eye movements in reading and information processing. Psychological Bulletin, 85(3), 618-660. doi:10.1080/13803395.2011.639298

Rayner, K. (1998). Eye movements in reading and information processing: 20 years of research. Psychological Bulletin, 124(3), 372-422. doi:10.1037/0033-2909.124.3. 372

Rayner, K. (2009). Eye movements and attention in reading, scene perception, and visual search. doi:10.1080/17470210902816461

Reeves, P. (1920). The response of the average pupil to the various intensities of light. Journal of the Optical Society of America, 4(2), 271-319. Retrieved from http: //www.opticsinfobase.org/viewmedia.cfm?id=47228\%7B\%5C\&\%7Dseq=0

Reinerman-Jones, L. E., Matthews, G., Langheim, L. K., \& Warm, J. S. (2011). Selection for vigilance assignments: A review and proposed new direction. Theoretical Issues in Ergonomics Science, 12(4), 273-296. doi:10.1080/14639221003622620

Reingold, E. M., Charness, N., Pomplun, M., \& Stampe, D. M. (2001). Visual span in expert chess players: Evidence from eye movements. Psychological Science, 12(1), 48-55.

Reingold, E. M., Loschky, L. C., McConkie, G. W., \& Stampe, D. M. (2003). Gazecontingent multiresolutional displays: An integrative review. Human Factors, 45(2), 307-328. doi:10.1518/hfes.45.2.307.27235

Richer, F. \& Beatty, J. (1985). Pupillary dilations in movement preparation and execution. Psychophysiology, 22(2), 204-207. doi:10.1111/j.1469-8986.1985.tb01587.x

Richer, F. \& Beatty, J. (1987). Contrasting effects of response uncertainty on the taskevoked pupillary response and reaction time. Psychophysiology, 24(3), 258-262. doi:doi:10.1111/j.1469-8986.1987.tb00291.x

Richer, F., Silverman, C., \& Beatty, J. (1983). Response selection and initiation in speeded reactions: A pupillometric analysis. Journal of Experimental Psychology: Human Perception and Performance, 9(3), 360-370. doi:10.1037/0096-1523.9.3. 360 
Roach, G. D., Dawson, D., \& Lamond, N. (2006). Can a shorter psychomotor vigilance task be used as a reasonable substitute for the ten-minute psychomotor vigilance task? Chronobiology International, 23(6), 1379-87. doi:10.1080/ 07420520601067931

Robertson, I. H., Manly, T., Andrade, J., Baddeley, B. T., \& Yiend, J. (1997). 'Oops!': Performance correlates of everyday attentional failures in traumatic brain injured and normal subjects. Neuropsychologia, 35(6), 747-758. doi:10.1016/S00283932(97)00015-8

Rohrbaugh, J. W., Stapleton, J. M., Parasuraman, R., Zubovic, E. A., Frowein, H. W., Varner, J. L., ... Linnoila, M. (1987). Dose-related effects of ethanol on visual sustained attention and event-related potentials. Alcohol, 4(4), 293-300. doi:10. 1016/0741-8329(87)90026-7

Rosenholtz, R., Li, Y., \& Nakano, L. (2007). Measuring visual clutter. Journal of Vision, 7(2), 17.1-22. doi:10.1167/7.2.17

Ross, H. A., Russell, P. N., \& Helton, W. S. (2014). Effects of breaks and goal switches on the vigilance decrement. Experimental Brain Research, 232(6), 1729-1737. doi:10.1007/s00221-014-3865-5

Rouder, J. N., Morey, R. D., Cowan, N., Zwilling, C. E., Morey, C. C., \& Pratte, M. S. (2008). An assessment of fixed-capacity models of visual working memory. Proceedings of the National Academy of Sciences, USA, 105(16), 5975-5979. doi:10.1073/pnas.0711295105

Roussel, B., Buguet, A., Bobillier, P., \& Jouvet, M. (1967). Locus ceruleus, paradoxal sleep, and cerebral noradrenaline. Comptes rendus des seances de la Societe de biologie et de ses filiales, 161(12), 2537. Retrieved from https://www.ncbi.nlm. nih.gov/pubmed/4302168

Rubinstein, J. (2001). Test and evaluation plan: X-ray image screener selection test. US Federal Aviation Administration.

Rucci, M., Iovin, R., Poletti, M., \& Santini, F. (2007). Miniature eye movements enhance fine spatial detail. Nature, 447(7146), 852-855. doi:10.1038/nature05866

Russo, M., Thomas, M. L., Thorne, D. R., Sing, H. C., Redmond, D. P., Rowland, L. M., ... Balkin, T. (2003). Oculomotor impairment during chronic partial sleep deprivation. Clinical Neurophysiology, 114(4), 723-736. doi:10.1016/S13882457(03)00008-7

Sagi, D. \& Julesz, B. (1985). Fast noninertial shifts of attention. Spatial Vision, 1(2), 141-149. doi:10.1163/156856885X00152 
Samuel, S., Kundel, H. L., Nodine, C. F., \& Toto, L. C. (1995). Mechanism of satisfaction of search: Eye position recordings in the reading of chest radiographs. Radiology, 194(3), 895-902. doi:10.1148/radiology.194.3.7862998

Samuels, E. R. \& Szabadi, E. U. (2008a). Functional neuroanatomy of the noradrenergic locus coeruleus: its roles in the regulation of arousal and autonomic function part I: Principles of functional organisation. Current Neuropharmacology, 6(3), 235-253. doi:10.2174/157015908785777229

Samuels, E. R. \& Szabadi, E. U. (2008b). Functional neuroanatomy of the noradrenergic locus coeruleus: its roles in the regulation of arousal and autonomic function part II: Physiological and pharmacological manipulations and pathological alterations of locus coeruleus activity in humans. Current Neuropharmacology, 6(3), 254285. doi:10.2174/157015908785777193

Sara, S. J. (1985a). Noradrenergic modulation of selective attention: Its role in memory retrieval. Annals of the New York Academy of Sciences, 444(1), 178-193. doi:10. 1111/j.1749-6632.1985.tb37588.x

Sara, S. J. (1985b). The locus coeruleus and cognitive function: Attempts to relate noradrenergic enhancement of signal/noise in the brain to behavior. Physiological Psychology, 13(3), 151-162. doi:10.3758/BF03326515

Sara, S. J. (2009). The locus coeruleus and noradrenergic modulation of cognition. Nature Reviews Neuroscience, 10(3), 211-23. doi:10.1038/nrn2573

Sara, S. J. \& Bouret, S. (2012). Orienting and reorienting: The locus coeruleus mediates cognition through arousal. Neuron, 76(1), 130-141. doi:10.1016/j.neuron.2012. 09.011

Sara, S. J. \& Segal, M. (1991). Plasticity of sensory responses of locus coeruleus neurons in the behaving rat: Implications for cognition. Progress in Brain Research, 88(100), 571-585. doi:10.1016/S0079-6123(08)63835-2

Sarter, N. B., Mumaw, R. J., \& Wickens, C. D. (2007). Pilots' monitoring strategies and performance on automated flight decks: An empirical study combining behavioral and eye-tracking data. Human Factors, 49(3), 347-357. doi:10.1518/ $001872007 X 196685$

Satchell, P. M. (1993). Cockpit monitoring and alerting systems. Brookfield, VT: Ashagate.

Sawin, D. A. \& Scerbo, M. W. (1995). Effects of instruction type and boredom proneness in vigilance: Implications for boredom and workload. Human Factors, 37(4), 752-765. doi:10.1518/001872095778995616 
Scheepers, C., Mohr, S., Fischer, M. H., \& Roberts, A. M. (2013). Listening to Limericks: A pupillometry investigation of perceivers' expectancy. PLoS ONE, 8(9), e74986. doi:10.1371/journal.pone.0074986

Schooler, J. W., Smallwood, J., Christoff, K., Handy, T. C., Reichle, E. D., \& Sayette, M. A. (2011). Meta-awareness, perceptual decoupling and the wandering mind. Trends in Cognitive Sciences, 15(7), 319-326. doi:10.1016/j.tics.2011.05.006

Schwaninger, A. \& Bolfing, A. (2008). The impact of image based factors and training on threat detection performance in X-ray screening. In Proceedings of the 3rd international conference on research in air transportation (pp. 317-324). Fairfax, VA. Retrieved from http://www. casra.ch/uploads/tx \% 7B \% 5C_ \%7Dtvpublications/SchBolHalHelBelHay2008\%7B\%5C_\%7D02.pdf

Scialfa, C. T. \& Joffe, K. M. (1998). Response times and eye movements in feature and conjunction search as a function of target eccentricity. Perception \& Psychophysics, 60(6), 1067-1082. doi:10.3758/BF03211940

Sewell, D. K., Lilburn, S. D., \& Smith, P. L. (2014). An information capacity limitation of visual short-term memory. Journal of Experimental Psychology: Human Perception and Performance, 40(6), 2214-2242. doi:10.1037/a0037744

Shannon, C. E. (1948). A mathematical theory of communication. The Bell System Technical Journal, 27, 379-423. doi:10.1145/584091.584093

Sheridan, T. B. (1970). On how often the supervisor should sample. IEEE Transactions on Systems Science \& Cybernetics, 6(2), 140-145. doi:10.1109/TSSC.1970. 300289

Sheridan, T. B. (2012). Human supervisory control. In Handbook of human factors and ergonomics (4th, pp. 990-1015). doi:10.1002/9781118131350.ch34

Shiffrin, R. M. \& Gardner, G. T. (1972). Visual processing capacity and attentional control. Journal of Experimental Psychology, 93(1), 72-82. doi:10.1037/h0032453

Shoenberger, R. W. (1967). Effects of vibration on complex psychomotor performance. Aerospace Medicine, 38, 1264-1269.

Simmers, J., Meyrand, P., \& Moulins, M. (1995). Modulation and dynamic specification of motor rhythm-generating circuits in crustacea. Journal of Physiology - Paris, 89(4-6), 195-208. doi:10.1016/0928-4257(96)83636-9

Simpson, H. M. \& Hale, S. M. (1969). Pupillary changes during a decision-making task. Perceptual and Motor Skills, 29(2), 495-498. doi:10.2466/pms.1969.29.2.495

Simpson, H. M. \& Molloy, F. M. (1971). Effects of audience anxiety on pupil size. Psychophysiology, 8(4), 491-497. 
Simpson, H. M. \& Paivio, A. (1966). Changes in pupil size during an imagery task without motor response involvement. Psychonomic Science, 5(10), 405-406. doi:10.3758/BF03328462

Sirois, S. \& Brisson, J. (2014). Pupillometry. Wiley Interdisciplinary Reviews: Cognitive Science, 679-692. doi:10.1002/wcs.1323

Smallwood, J. (2003). The effects of block duration and task demands on the experience of task unrelated thought. Imagination, Cognition \& Personality, 22(1), 13-31. doi:10.2190/TBML-N8JN-W5YB-4L9R

Smallwood, J. (2010). Why the global availability of mind wandering necessitates resource competition: Reply to McVay and Kane (2010). Psychological Bulletin, 136(2), 202-207. doi:10.1037/a0018673

Smallwood, J., Brown, K. S., Baird, B., Mrazek, M. D., Franklin, M. S., \& Schooler, J. W. (2012). Insulation for daydreams: A role for tonic norepinephrine in the facilitation of internally guided thought. PLOS ONE, 7(4), e33706. doi:10.1371/ journal.pone.0033706

Smallwood, J., Brown, K. S., Tipper, C., Giesbrecht, B., Franklin, M. S., Mrazek, M. D., ... Schooler, J. W. (2011). Pupillometric evidence for the decoupling of attention from perceptual input during offline thought. PLoS ONE, 6(3), e18298. doi:10.1371/journal.pone.0018298

Smallwood, J., Davies, J. B., Heim, D., Finnigan, F., Sudberry, M., O’Connor, R., \& Obonsawin, M. (2004). Subjective experience and the attentional lapse: Task engagement and disengagement during sustained attention. Consciousness and Cognition, 13(4), 657-690. doi:10.1016/j.concog.2004.06.003

Smallwood, J. \& Schooler, J. W. (2006). The restless mind. Psychology of Consciousness: Theory, Research, and Practice, 132(6), 946-958. doi:10.1037/00332909.132.6.946

Smallwood, J. \& Schooler, J. W. (2015). The science of mind wandering: Empirically navigating the stream of consciousness. Annual Review of Psychology, 66(1), 487-518. doi:10.1146/annurev-psych-010814-015331

Smit, A. S., Eling, P. A. T. M., \& Coenen, A. M. L. (2004). Mental effort causes vigilance decrease due to resource depletion. Acta Psychologica, 115(1), 35-42. doi:10.1016/j.actpsy.2003.11.001

Smith, P. L. \& Sewell, D. K. (2013). A competitive interaction theory of attentional selection and decision making in brief, multielement displays. Psychological Review, 120(3), 589-627. doi:10.1037/a0033140 
Smith, P. L., Sewell, D. K., \& Lilburn, S. D. (2015). From shunting inhibition to dynamic normalization: Attentional selection and decision-making in brief visual displays. Vision Research, 116, 219-240. doi:10.1016/j.visres.2014.11.001

Smith, R. L. (1966). Monotony and motivation: A theory of vigilance. Los Angeles: Dunlop.

Snyder, J. J. \& Kingstone, A. (2000). Inhibition of return and visual search: How many separate loci are inhibited? Perception \& Psychophysics, 62(3), 452-458. doi:10.3758/BF03212097

Sokolov, E. N. (1963). Higher nervous functions: The orienting reflex. Annual Review of Physiology, 25, 545-580. doi:10.1146/annurev.ph.25.030163.002553

Sparks, D. L. (1986). Translation of sensory signals into commands for control of saccadic eye movements: Role of primate superior colliculus. Physiological Reviews, 66(1), 118-171. Retrieved from http://www.ncbi.nlm.nih.gov/pubmed/ 3511480

Sperling, G. (1960). The information available in brief visual presentations. Psychological Monographs: General and Applied, 11(48), 1-29.

SR Research Ltd. (2010). EyeLink user manual. Version 1.5.2. Mississauga, Canada.

Stanislaw, H. \& Todorov, N. (1999). Calculation of signal detection theory measures. Behavior Research Methods, Instruments, \& Computers, 31(1), 137-149. doi:10. 3758/BF03207704

Starc, M., Anticevic, A., \& Repovš, G. (2017). Fine-grained versus categorical: Pupil size differentiates between strategies for spatial working memory performance. Psychophysiology, 54(5), 724-735. doi:10.1111/psyp.12828

Starker, I. \& Bolt, R. a. (1990). A gaze-responsive self-disclosing display. In Proceedings of the sigchi conference on human factors in computing systems empowering people - chi '90 (April, pp. 3-10). doi:10.1145/97243.97245

Steinhauer, S. R. (2002). Cognition, psychopathology, and recent pupil studies. Retrieved June 5, 2017, from www.wpic.pitt.edu/research/biometrics/Publications/ PupilWeb.htm

Steinhauer, S. R. \& Hakerem, G. (1992). The pupillary response in cognitive psychophysiology and schizophrenia. Annals of the New York Academy of Sciences, 658(1), 182-204. doi:10.1111/j.1749-6632.1992.tb22845.x

Steinhauer, S. R., Siegle, G. J., Condray, R., \& Pless, M. (2004). Sympathetic and parasympathetic innervation of pupillary dilation during sustained processing. 
International Journal of Psychophysiology, 52(1), 77-86. doi:10.1016/j.ijpsycho. 2003.12.005

Steinhauer, S. R. \& Zubin, J. (1982). Vulnerability to schizophrenia: Information processing in the pupil and event-related potential. In E. Usdin \& I. Hanin (Eds.), Biological markers in psvchiatry and neurology (pp. 371-385). Oxford: Pergamon Press.

Stephens, D. W. \& Krebs, J. R. (1986). Foraging theory. Princeton, NJ: Princeton University Press.

Stern, J. A., Boyer, D., \& Schroeder, D. J. (1994). Blink rate: A possible measure of fatigue. Human Factors, 36(2), 285-297. doi:10.1177/001872089403600209

Stoll, J., Chatelle, C., Carter, O. L., Koch, C., Laureys, S., \& Einhäuser, W. (2013). Pupil responses allow communication in locked-in syndrome patients. Current Biology, 23(15), R647-8. doi:10.1016/j.cub.2013.06.011

Strauch, C., Greiter, L., \& Huckauf, A. (2017a). Pupil-assisted target selection (PATS). In R. Bernhaupt, G. Dalvi, A. K. Joshi, D. O. Balkrishan, \& M. Winckler (Eds.), Human-computer interaction - interact 2017 (Vol. 10514, pp. 297-312). Cham. doi:10.1007/978-3-319-67687-6_20

Strauch, C., Greiter, L., \& Huckauf, A. (2017b). Towards pupil-assisted target selection in natural settings: Introducing an on-screen keyboard. In R. Bernhaupt, G. Dalvi, A. K. Joshi, D. O. Balkrishan, \& M. Winckler (Eds.), Human-computer interaction - interact 2017 (Vol. 10514, pp. 534-543). Cham. doi:10.1007/978-3-319-676845

Stroop, J. R. (1935). Studies of interference in serial verbal reactions. Journal of Experimental Psychology, 18(6), 643. doi:52,281-302

Takeda, Y. \& Yagi, A. (2000). Inhibitory tagging in visual search can be found if search stimuli remain visible. Perception \& Psychophysics, 62(5), 927-934. doi:10.3758/ BF03212078

Tanriverdi, V. \& Jacob, R. J. K. (2000). Interacting with eye movements in virtual environments. Proceedings of the ACM CHI'OO Conference on Human Factors in Computing Systems, 265-272. doi:10.1145/332040.332443

Tarriere, C., Hartemann, F., \& Niarfeix, M. (1966). Influence of cigarette smoking upon the course of performance in a watchkeeping task. La Travail Humain, 29, 1-21.

Teasdale, J. D., Dritschel, B. H., Taylor, M. J., Proctor, L., Lloyd, C. A., NimmoSmith, I., \& Baddeley, A. D. (1995). Stimulus-independent thought depends on 
central executive resources. Memory \& Cognition, 23(5), 551-559. doi:10.3758/ BF03197257

Thomson, D. R., Besner, D., \& Smilek, D. (2015). A resource-control account of sustained attention: Evidence from mind wandering and vigilance paradigms. Perspectives on Psychological Science, 10(1), 82-96. doi:10.1177/1745691614556681

Thorne, D. R., Johnson, D. E., Redmond, D. P., Sing, H. C., Belenky, G., \& Shapiro, J. M. (2005). The Walter Reed palm-held psychomotor vigilance test. Behavior Research Methods, 37(1), 111-118. doi:10.3758/BF03206404

Tipper, S. P., Driver, J., \& Weaver, B. (1991). Object-centred inhibition of return of visual attention. The Quarterly Journal of Experimental Psychology, 43A(2), 289-298. doi:10.1080/14640749108400971

Todd, J. J. \& Marois, R. (2004). Capacity limit of visual short-term memory in human posterior parietal cortex. Nature, 428(6984), 751-754. doi:10.1038/nature02466

Treisman, A. M. (1988). Features and objects: The fourteenth Bartlett memorial lecture. The Quarterly Journal of Experimental Psychology, 40A(2), 201-237. doi:10. 1080/02724988843000104

Treisman, A. M. (1991). Search, similarity, and integration of features between and within dimensions. Journal of Experimental Psychology: Human Perception and Performance, 17(3), 652-676. doi:10.1037/0096-1523.17.3.652

Treisman, A. M. (1993). The perception of features and objects. In Attention, selection, awareness and control (pp. 5-35). Oxford: Clarendon Press.

Treisman, A. M. \& Gelade, G. (1980). A feature-integration theory of attention. Cognitive Psychology, 12(1), 97-136. Retrieved from http://www.ncbi.nlm.nih.gov/ pubmed/7351125

Treisman, A. M. \& Sato, S. (1990). Conjunction search revisited. Journal of Experimental Psychology: Human Perception and Performance, 75(3), 459-478. doi:10.1037/0096-1523.16.3.459

Treisman, A. M. \& Souther, J. (1985). Search asymmetry: A diagnostic for preattentive processing of separable features. Journal of Experimental Psychology: General, 114(3), 285-310. doi:10.1037/0096-3445.114.3.285

Trejo, L. J., Rosipal, R., \& Matthews, B. (2006). Brain-computer interfaces for 1-D and 2-D cursor control: Designs using volitional control of the EEG spectrum or steady-state visual evoked potentials. In Ieee transactions on neural systems and rehabilitation engineering (Vol. 14, 2, pp. 225-229). doi:10.1109/TNSRE.2006. 875578 
Trukenbrod, H. A. \& Engbert, R. (2007). Oculomotor control in a sequential search task. Vision Research, 47(18), 2426-2443. doi:10.1016/j.visres.2007.05.010

Tuddenham, W. J. (1962). Visual search, image organization, and reader error in roentgen diagnosis. Radiology, 78(5), 694-704. doi:10.1148/78.5.694

Turing, A. M. (1950). Computing machinery and intelligence. Mind, 59(236), 433-460. Retrieved from http://www.jstor.org/stable/2251299

Ukai, K. (1985). Spatial pattern as a stimulus to the pupillary system. Journal of the Optical Society of America: A, 2(7), 1094-1100. doi:10.1364/JOSAA.2.001094

Underwood, G., Chapman, P. R., Bowden, K., \& Crundall, D. (2002). Visual search while driving: Skill and awareness during inspection of the scene. Transportation Research Part F: Traffic Psychology and Behaviour, 5(2), 87-97. doi:10.1016/ S1369-8478(02)00008-6

Unsworth, N. \& Engle, R. W. (2007). The nature of individual differences in working memory capacity: Active maintenance in primary memory and controlled search from secondary memory. Psychological Review, 114(1), 104-132. doi:10.1037/ 0033-295X.114.1.104

Unsworth, N. \& Robison, M. K. (2016). Pupillary correlates of lapses of sustained attention. Cognitive, Affective \& Behavioral Neuroscience, 16(April), 601-615. doi:10.3758/s13415-016-0417-4

Unsworth, N. \& Robison, M. K. (2017). Pupillary correlates of covert shifts of attention during working memory maintenance. Attention, Perception, \& Psychophysics, l(2013). doi:10.3758/s13414-016-1272-7

Urai, A. E., Braun, A., \& Donner, T. H. (2017). Pupil-linked arousal is driven by decision uncertainty and alters serial choice bias. Nature Communications, 8 , 14637. doi:10.1038/ncomms 14637

Usher, M., Cohen, J. D., Servan-Schreiber, D., Rajkowski, J., \& Aston-Jones, G. (1999). The role of locus coeruleus in the regulation of cognitive performance. Science, 283(5401), 549-554. doi:10.1126/science.283.5401.549

Valeriani, D., Poli, R., \& Cinel, C. (2016). Enhancement of group perception via a collaborative brain-computer interface. IEEE transactions on biomedical engineering, 9294(100), 1-11. doi:10.1109/TBME.2016.2598875

Valeriani, D., Poli, R., Valeriani, D., Poli, R., \& Cinel, C. (2015). A collaborative brain-computer interface to improve human performance in a visual search task. In 7th annual international ieee embs conference on neural engineering (May). Montpellier, France. doi:10.1109/NER.2015.7146599 
van den Berg, R., Shin, H., Chou, W.-C., George, R., \& Ma, W. J. (2012). Variability in encoding precision accounts for visual short-term memory limitations. Proceedings of the National Academy of Sciences, USA, 109(22), 8780-8785. doi:10.1073/pnas.1117465109

van den Brink, R. L., Murphy, P. R., \& Nieuwenhuis, S. (2016). Pupil diameter tracks lapses of attention. PLoS ONE, 11(10), 1-16. doi:10.1371/journal.pone.0165274

van der Meer, E., Friedrich, M., Nuthmann, A., Stelzel, C., \& Kuchinke, L. (2003). Picture-word matching: flexibility in conceptual memory and pupillary responses. Psychophysiology, 40(6), 904-913. doi:10.1111/1469-8986.00108

Van Dongen, H. P. A. \& Dinges, D. F. (2005). Sleep, circadian rhythms, and psychomotor vigilance. Clinics in Sports Medicine, 24(2), 237-249. doi:10.1016/j.csm. 2004.12.007

Van Dongen, H. P. A., Price, N. J., Mullington, J. M., Szuba, M. P., Kapoor, S. C., \& Dinges, D. F. (2001). Caffeine eliminates psychomotor vigilance deficits from sleep inertia. Sleep, 24(7), 813-819. doi:10.1093/sleep/24.7.813

Van Orden, K. F., Jung, T. P., \& Makeig, S. (2000). Combined eye activity measures accurately estimate changes in sustained visual task performance. Biological Psychology, 52(3), 221-240. doi:10.1016/S0301-0511(99)00043-5

van Zoest, W., Lleras, A., Kingstone, A., \& Enns, J. T. (2007). In sight, out of mind: The role of eye movements in the rapid resumption of visual search. Perception \& Psychophysics, 69(7), 1204-1217. doi:10.3758/BF03193956

Vankov, A., Hervé-Minvielle, A., \& Sara, S. J. (1995). Response to Novelty and its Rapid Habituation in Locus Coeruleus Neurons of the Freely Exploring Rat. European Journal of Neuroscience, 7(6), 1180-1187. doi:10.1111/j .14609568.1995.tb01108.x

Varazzani, C., San-Galli, A., Gilardeau, S., \& Bouret, S. (2015). Noradrenaline and dopamine neurons in the reward/effort trade-off: A direct electrophysiological comparison in behaving monkeys. The Journal of Neuroscience, 35(7866), 7877. doi:10.1523/JNEUROSCI.0454-15.2015

Verde, M. E., MacMillan, N. A., \& Rotello, C. M. (2006). Measures of sensitivity based on a single hit rate and false alarm rate: The accuracy, precision, and robustness of d', Az, and A'. Perception \& Psychophysics, 68(4), 643-654. doi:10.3758/ BF03208765

Verghese, P. (2001). Visual search and attention: A signal detection theory approach. Neuron, 31(4), 523-535. doi:10.1016/S0896-6273(01)00392-0 
Vertegaal, R. (1999). The GAZE groupware system: Mediating joint attention in multiparty communication and collaboration. In Proceedings of the acm chi'99 conference on human factors in computing systems (pp. 294-301). Pittsburgh, PA: ACM. doi:10.1145/302979.303065

Vidal, J. J. (1977). Real-time detection of brain events in EEG. Proceedings of the IEEE, 65(5), 633-641. doi:10.1109/PROC.1977.10542

Vinge, V. (1993). The coming technological singularity: How to survive in the posthuman era. In Vision 21: Interdisciplinary science and engineering in the era of cyberspace (Vol. 21, pp. 11-22). Westlake, Ohio: NASA. Retrieved from https://ntrs.nasa.gov/search.jsp?R=19940022856

Võ, M. L.-H., Jacobs, A. M., Kuchinke, L., Hofmann, M., Conrad, M., Schacht, A., \& Hutzler, F. (2008). The coupling of emotion and cognition in the eye: Introducing the pupil old / new effect. Psychophysiology, 45, 130-140. doi:10.1111/j.14698986.2007.00606.x

Võ, M. L.-H. \& Wolfe, J. M. (2012). When does repeated search in scenes involve memory? Looking at versus looking for objects in scenes. Journal of Experimental Psychology: Human Perception and Performance, 38(1), 23-41. doi:10.1037/ a0024147

Vogel, E. K. \& Machizawa, M. G. (2004). Neural activity predicts individual differences in visual working memory capacity. Nature, 428(6984), 748-751. doi:10.1038/ nature 02447

Vogel, E. K., McCollough, A. W., \& Machizawa, M. G. (2005). Neural measures reveal individual differences in controlling access to working memory. Nature, 438(7067), 500-503. doi:10.1038/nature04171

Vogel, E. K., Woodman, G. F., \& Luck, S. J. (2001). Storage of features, conjunctions and objects in visual working memory. doi:10.1037//0096-1523.27.1.92

Wade, N. J. \& Tatler, B. W. (2005). The moving tablet of the eye: The origins of modern eye movement research. USA: Oxford University Press. doi:10.1093/acprof: oso/9780198566175.001.0001

Wade, N. J. \& Tatler, B. W. (2012). Origins and applications of eye movement research. In The oxford handbook of eye movements (pp. 17-46). Oxford, UK: Oxford University Press. doi:10.1093/oxfordhb/9780199539789.013.0002

Walker, R., Deubel, H., Schneider, W. X., \& Findlay, J. M. (1997). Effect of remote distractors on saccade programming: evidence for an extended fixation zone. Journal of Neurophysiology, 78(2), 1108-1119. 
Walter, W. G., Cooper, R., Aldridge, V. J., McCallum, W. C., \& Winter, A. L. (1964). Contingent negative variation: An electric sign of sensori-motor association and expectancy in the human brain. Nature, 203(4943), 380-384. doi:10.1038/ 203380a0

Wang, C.-A., Boehnke, S. E., Itti, L., \& Munoz, D. P. (2014). Transient pupil response is modulated by contrast-based saliency. The Journal of Neuroscience, 34(2), 408-417. doi:10.1523/JNEUROSCI.3550-13.2014

Wang, C.-A., Boehnke, S. E., White, B. J., \& Munoz, D. P. (2012). Microstimulation of the monkey superior colliculus induces pupil dilation without evoking saccades. The Journal of Neuroscience, 32(11), 3629-3636. doi:10.1523/JNEUROSCI. 5512-11.2012

Wang, C.-A. \& Munoz, D. P. (2014). Modulation of stimulus contrast on the human pupil orienting response. European Journal of Neuroscience, 40(5), 2822-32. doi:10.1111/ejn.12641

Wang, C.-A. \& Munoz, D. P. (2015). A circuit for pupil orienting responses: Implications for cognitive modulation of pupil size. Current Opinion in Neurobiology, 33, 134140. doi:10.1016/j.conb.2015.03.018

Wang, J. T.-y., Spezio, M., \& Camerer, C. F. (2010). Pinocchio's pupil: Using eyetracking and pupil dilation to understand truth-telling and deception in games. The American Economic Review, 3(3), 984-1007. doi:10.1257/aer.100.3.984

Warm, J. S. (1993). Vigilance and target detection. In C. D. Wickens \& B. M. Huey (Eds.), Workload transition: Implications for individual and team performance (pp. 139-170). Washington DC: National Research Council.

Warm, J. S., Dember, W. N., \& Hancock, P. A. (1996). Vigilance and workload in automated systems. In Automation and human performance: Theory and applications (pp. 183-200).

Warm, J. S., Epps, B. D., \& Ferguson, R. P. (1974). Effects of knowledge of results and signal regularity on vigilance performance. Bulletin of the Psychonomic Society, 4(4A), 272-274. doi:10.3758/BF03336723

Warm, J. S. \& Jerison, H. J. (1984). The psychophysics of vigilance. In J. S. Warm (Ed.), Sustained attention in human performance (pp. 15-59). Chichester: John Wiley \& Sons.

Warm, J. S., Loeb, M., \& Alluisi, E. A. (1970). Variations in watchkeeping performance as a function of the rate and duration of visual signals. Perception \& Psychophysics, 7(2), 97. 
Warm, J. S., Parasuraman, R., \& Matthews, G. (2008). Vigilance requires hard mental work and is stressful. Human Factors, 50(3), 433-441. doi:10.1518/001872008X312152

Watson, A. B. \& Yellott, J. I. (2012). A unified formula for light-adapted pupil size. Journal of Vision, 12, 1-16. doi:10.1167/12.10.12.Introduction

Watson, D. G. \& Inglis, M. (2007). Eye movements and time-based selection: Where do the eyes go in preview search? Psychonomic Bulletin \& Review, 14(5), 852-857. doi:10.3758/BF03194111

Waugh, N. C. \& Norman, D. A. (1965). Primary memory. Psychological Review, 72(2), 89-104. doi:10.1037/h0021797

Webb, H. E., McMinn, D. R., Garten, R. S., Beckman, J. L., Kamimori, G. H., \& Acevedo, E. O. (2010). Cardiorespiratory responses of firefighters to a computerized fire strategies and tactics drill during physical activity. Applied Ergonomics, 41(3), 376-381. doi:10.1016/j.apergo.2009.08.003

Weichselgartner, E. \& Sperling, G. (1987). Dynamics of automatic and controlled visual attention. Science, 238(4828), 778-780. doi:10.1126/science.3672124

Weinger, M. B. \& Englund, C. E. (1990). Ergonomic and human factors affecting anesthetic vigilance and monitoring performance in the operating room environment. Anesthesiology, 73, 995-1021.

Weiskopf, N., Scharnowski, F., Veit, R., Goebel, R., Birbaumer, N., \& Mathiak, K. (2004). Self-regulation of local brain activity using real-time functional magnetic resonance imaging (fMRI). Journal of Physiology-Paris, 98(4), 357-373. doi:10. 1016/j.jphysparis.2005.09.019

Weiskopf, N., Veit, R., Erb, M., Mathiak, K., Grodd, W., Goebel, R., \& Birbaumer, N. (2003). Physiological self-regulation of regional brain activity using real-time functional magnetic resonance imaging (fMRI): Methodology and exemplary data. NeuroImage , 19, 577-586. doi:10.1016/S1053-8119(03)00145-9

Wheeler, M. E. \& Treisman, A. M. (2002). Binding in short-term visual memory. Journal of Experimental Psychology: General, 131(1), 48-64. doi:10.1037//00963445.131.1.48

White, B. J. \& Munoz, D. P. (2011). The superior colliculus. In S. P. Liversedge, I. D. Gilchrist, \& S. Everling (Eds.), The oxford handbook of eye movements (pp. 195213). Oxford: Oxford University Press.

Wickens, T. D. (2002). Elementary signal detection theory. New York: Oxford University Press. doi:10.1093/acprof:oso/9780195092509.001.0001 
Widdel, H. (1984). Operational problems in analysing eye movements. In A. Gale \& F. Johnson (Eds.), Theoretical and applied aspects of eye movement research (pp. 21-29). New York: Elsevier.

Wiener, E. L. (1984). Vigilance and inspection. In Sustained attention in human performance (pp. 207-246). Chichester, UK: Wiley.

Wiener, E. L. (1987). Application of vigilance research: Rare, medium, or well done? Human Factors, 29(6), 725-736. doi:10.1177/001872088702900611

Wierda, S. M., van Rijn, H., Taatgen, N. A., \& Martens, S. (2012). Pupil dilation deconvolution reveals the dynamics of attention at high temporal resolution. Proceedings of the National Academy of Sciences, USA, 109(22), 8456-8460. doi:10.1073/pnas.1201858109

Wilhelm, B., Giedke, H., Lüdtke, H., Bittner, E., Hofmann, A., \& Wilhelm, H. (2001). Daytime variations in central nervous system activation measured by a pupillographic sleepiness test. Journal of Sleep Research, 10(1), 1-7. doi:10.1046/j.13652869.2001.00239.x

Wilhelm, H., Lüdtke, H., \& Wilhelm, B. (1998). Pupillographic sleepiness testing in hypersomniacs and normals. Graefe's Archive for Clinical and Experimental Ophthalmology, 236(10), 725-729. doi:10.1007/s004170050149

Wilkinson, R. T. (1960). The effect of lack of sleep on visual watch-keeping. The Quarterly Journal of Experimental Psychology, 12(1), 36-40. doi:10.1080/ 17470216008416698

Wilkinson, R. T. \& Gray, R. (1974). Effects of duration of vertical vibration beyond the proposed ISO "fatigue decreased proficiency" time on the performance of various tasks. In Agard conference on vibration and combined stresses in advanced systems. Oslo.

Wilkinson, R. T. \& Houghton, D. (1982). Field test of arousal: a portable reaction timer with data storage. Human Factors, 24(4), 487-493. doi:10.1177/001872088202400409

Willenbockel, V., Sadr, J., Fiset, D., Horne, G. O., Gosselin, F., \& Tanaka, J. W. (2010). Controlling low-level image properties: The SHINE toolbox. Behavior Research Methods, 42(3), 671-84. doi:10.3758/BRM.42.3.671

Williams, C. C. \& Pollatsek, A. (2007). Searching for a O in a array of Cs: Eye movements track moment-to-moment processing in visual search. Perception \& Psychophysics, 69(3), 372-381. doi:10.3758/BF03193758 
Williams, H. L., Lubin, A., \& Goodnow, J. J. (1959). Impaired performance with acute sleep loss. Psychological Monographs: General and Applied, 73(14), 1-26. doi:10.1037/h0093749

Williges, R. C. (1971). The role of payoffs and signal ratios in criterion changes during a monitoring task. Human Factors, 13(3), 261-267. doi:10.1177/001872087101300307

Wilson, G. F. (1990). Classification of flight segment using pilot and WSO physiological data. In Proceedings of the human factors society 34th annual meeting (Vol. 34, 1, pp. 109-111).

Wilson, G. F. \& Skelly, J. (1987). Wilson Brad. In Proceedings of the human factors society 31st annual meeting (1987, pp. 779-783).

Wolfe, J. M. (1994a). Guided search 2.0: A revised model of visual search. Psychonomic Bulletin \& Review, 1(2), 202-38. doi:10.3758/BF03200774

Wolfe, J. M. (1994b). Visual search in continuous, naturalistic stimuli. Vision Research, 34(9), 1187-1195. doi:10.1080/1461670032000174783

Wolfe, J. M. (1998a). Visual Search. In H. Pashler (Ed.), Attention (pp. 13-73). Hove, UK: Psychology Press. doi:10.1016/j.tics.2010.12.001

Wolfe, J. M. (1998b). What can 1 million trials tell us about visual search? Psychological Science, 9(1), 33-39.

Wolfe, J. M. (2003). Moving towards solutions to some enduring controversies in visual search. Trends in Cognitive Sciences, 7(2), 70-76. doi:10.1016/S13646613(02)00024-4

Wolfe, J. M. (2006). Guided search 4.0: Current progress with a model of visual search. In Integrated models of cognitive systems (Vol. 1, pp. 99-119). New York: Oxford University Press. doi:10.1167/1.3.349

Wolfe, J. M. (2013). When is it time to move to the next raspberry bush? Foraging rules in human visual search. Journal of Vision, 13(3), 10. doi:10.1167/13.3.10

Wolfe, J. M., Brunelli, D. N., Rubinstein, J., \& Horowitz, T. S. (2013). Prevalence effects in newly trained airport checkpoint screeners: Trained observers miss rare targets, too. Journal of Vision, 13(3), 33-33. doi:10.1167/13.3.33

Wolfe, J. M., Cain, M., Ehinger, K. A., \& Drew, T. (2015). Guided search 5.0: Meeting the challenge of hybrid search and multiple-target foraging. Journal of Vision, 15(12), 1106. doi:10.1167/15.12.1106

Wolfe, J. M., Cave, K. R., \& Franzel, S. L. (1989). Guided search: An alternative to the feature integration model for visual search. Journal of Experimental Psychology: 
Human Perception and Performance, 15(3), 419-33. Retrieved from http://www. ncbi.nlm.nih.gov/pubmed/2527952

Wolfe, J. M. \& Gancarz, G. (1997). Guided search 3.0: A model of visual search catches up with jay enoch 40 years later. In Basic and clinical applications of vision science (60, pp. 189-192).

Wolfe, J. M. \& Horowitz, T. S. (2004). What attributes guide the deployment of visual attention and how do they do it? Nature Reviews Neuroscience, 5(June), 1-7.

Wolfe, J. M. \& Horowitz, T. S. (2017). Five factors that guide attention in visual search. Nature Human Behaviour, 1(3), 0058. doi:10.1038/s41562-017-0058

Wolfe, J. M., Horowitz, T. S., \& Kenner, N. M. (2005). Rare items often missed in visual searches. Nature, 435(May), 6-7.

Wolfe, J. M., Horowitz, T. S., Van Wert, M. J., Kenner, N. M., Place, S. S., \& Kibbi, N. (2007). Low target prevalence is a stubborn source of errors in visual search tasks. Journal of Experimental Psychology: General, 136(4), 623-638. doi:10.1037/ 0096-3445.136.4.623

Wolfe, J. M., Palmer, E. M., \& Horowitz, T. S. (2010). Reaction time distributions constrain models of visual search. Vision Research, 50(14), 1304-1311. doi:10. 1016/j.visres.2009.11.002

Wolfe, J. M. \& Pokorny, C. W. (1990). Inhibitory tagging in visual search: A failure to replicate. Perception \& Psychophysics, 48(4), 357-362. doi:10.3758/BF03206686

Wolfe, J. M. \& Van Wert, M. J. (2010). Varying target prevalence reveals two dissociable decision criteria in visual Search. Current Biology, 20(2), 121-124. doi:10.1016/j. cub.2009.11.066

Wolff, M. J., Scholz, S., Akyürek, E. G., \& van Rijn, H. (2015). Two visual targets for the price of one? Pupil dilation shows reduced mental effort through temporal integration. Psychonomic Bulletin \& Review, 22(1), 251-257. doi:10.3758/ s13423-014-0667-5. arXiv: 1011.1669

Wolpaw, J. R. \& McFarland, D. J. (2004). Control of a two-dimensional movement signal by a noninvasive brain-computer interface in humans. Proceedings of the National Academy of Sciences, USA, 101(51), 17849-17854. doi:10.1073/pnas. 0403504101

Wolpaw, J. R., McFarland, D. J., Neat, G. W., \& Forneris, C. A. (1991). An EEG-based brain-computer interface for cursor control. Electroencephalography and Clinical Neurophysiology, 78(3), 252-259. doi:10.1016/0013-4694(91)90040-B 
Woodhouse, J. M. (1975). The effect of pupil size on grating detection at various contrast levels. Vision Research, 15(6), 645-648. doi:10.1016/0042-6989(75)90278-3

Woodhouse, J. M. \& Campbell, F. W. (1975). The role of the pupil light reflex in aiding adaptation to the dark. Vision Research, 15(6), 649-653. doi:10.1016/00426989(75)90279-5

Wyatt, H. J. (1995). The form of the human pupil. Vision Research, 35(14), 2021-36. Retrieved from http://www.ncbi.nlm.nih.gov/pubmed/7660606

Xu, Y. \& Chun, M. M. (2006). Dissociable neural mechanisms supporting visual shortterm memory for objects. Nature, 440(7080), 91-5. doi:10.1038/nature04262

Yantis, S. (1993). Stimulus-driven attentional capture and attentional control settings. Journal of Experimental Psychology: Human Perception and Performance, 19(3), 676-81. doi:10.1037/0096-1523.19.3.676

Yarbus, A. (1967). Eye movements and vision. New York: Plenum Press.

Yerkes, R. M. \& Dodson, J. D. (1908). The relation of strength of stimulus to rapidity of habit-formation. Journal of Comparative Neurology and Psychology, 18(5), 459-482. doi:10.1002/cne.920180503

Yoss, R. E., Moyer, N. J., \& Hollenhorst, R. W. (1970a). Hippus and other spontaneous rhythmic pupillary waves. American Journal of Ophthalmology, 70(6), 935-41. doi:10.1016/0002-9394(70)92470-0

Yoss, R. E., Moyer, N. J., \& Hollenhorst, R. W. (1970b). Pupil size and spontaneous pupillary waves associated with alertness, drowsiness, and sleep. Neurology, 20(6), 545-554. doi:10.1212/WNL.20.6.545

Yu, A. J. \& Dayan, P. (2005). Uncertainty, neuromodulation, and attention. Neuron, 46(4), 681-692. doi:10.1016/j.neuron.2005.04.026

Zeki, S. M. (1976). The functional organization of projections from striate to prestriate visual cortex in the rhesus monkey. In Cold spring harbor symposia on quantitative biology (Vol. 40, pp. 591-600). Cold Spring Harbour Laboratory Press. doi:10.1101/SQB.1976.040.01.055

Zelinsky, G. J. (2008). A theory of eye movements during target acquisition. Psychological Review, 115(4), 787-835. doi:10.1037/a0013118

Zelinsky, G. J., Rao, R. P. N., Hayhoe, M. M., \& Ballard, D. H. (1997). Eye movements reveal the spatiotemporal dynamics of visual search. Psychological Science, 8(6), $448-453$. 
Zhang, J., Fougnie, D., Gong, X., Alvarez, G. A., \& Wolfe, J. M. (2015). Winter is coming: How humans forage in a temporally structured environment. Journal of Vision, 15(11), 1-11. doi:10.1167/14.10.913

Zils, E., Sprenger, A., Heide, W., Born, J., \& Gais, S. (2005). Differential effects of sleep deprivation on saccadic eye movements. Sleep, 28, 1109-1115. 
